\title{
The role of astrocytic RFX4_v3 in demyelination
}

\author{
Dissertation \\ For the award of the degree \\ "Doctor rerum naturalium (Dr. rer. nat.)" \\ of the Georg-August-University Goettingen \\ In the PhD program Molecular Medicine \\ of the Georg-August University School of Science (GAUSS)
}

Submitted by

Franziska Paap

born in

Leinefelde, Germany

Goettingen, 2015 


\section{Members of the Thesis Committee}

\section{Supervisor (Reviewer)}

Prof. Dr. Wolfgang Brück

Department of Neuropathology

University Medical Center, Georg-August-University Goettingen

\section{Second Member of the Thesis Committee (Reviewer)}

Prof. Dr. Mikael Simons

Department of Cellular Neuroscience

University Medical Center, Georg-August-University Goettingen

\section{Third Member of the Thesis Committee}

Prof. Dr. Dr. Hannelore Ehrenreich

Department of Clinical Neuroscience

Max Planck Institut for Experimental Medicine, Goettingen

\section{Members of the Examination Board}

Prof. Dr. Christine Stadelmann Nessler

Department of Neuropathology

University Medical Center, Georg-August-University Goettingen

Dr. Francesca Odoardi

Institute for Multiple Sclerosis Research

University Medical Center, Georg-August-University Goettingen

Prof. Dr. Martin Weber

Departments of Neuropathology and Neurology

University Medical Center, Georg-August-University Goettingen 
Meinem lieben Tjebbe 


\section{Affidavit}

I hereby declare that my dissertation entitled "The role of astrocytic RFX4_v3 in demyelination" has been written independently with no other sources and aids than quoted.

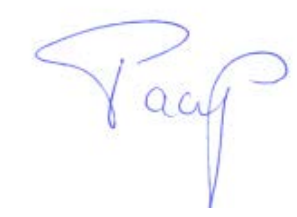

\section{Franziska Paap}

Goettingen, Februar 2015 


\section{Publications}

\section{Original Articles}

Jedrusik-Bode M, Studencka M, Smolka C, Baumann T, Schmidt H, Kampf J, Paap F, Martin S, Tazi J, Müller KM, Krüger M, Braun T, Bober E. (2013) The sirtuin SIRT6 regulates stress granule formation in C. elegans and mammals. J Cell Sci. 126, 5166-77.

Lescher J, Paap F, Schultz V, Redenbach L, Scheidt U, Rosewich H, Nessler S, Fuchs E, Gärtner J, Brück W, Junker A. (2012) MicroRNA regulation in experimental autoimmune encephalomyelitis in mice and marmosets resembles regulation in human multiple sclerosis lesions. J. Neuroimmunol. 246, 27-33.

Wirth M, Paap F, Fischle W, Wenzel D, Agafonov DE, Samatov TR, Wisniewski JR, Jedrusik-Bode M. (2009) HIS-24 linker histone and SIR-2.1 deacetylase induce H3K27me3 in the Caenorhabditis elegans germ line. Mol Cell Biol. 29, 3700-9. 


\section{Acknowledgements}

Abstract

III

List of Figures

Abbreviations $\quad$ VIII

1 Introduction 1

$\begin{array}{lll}1.1 & \text { Multiple sclerosis } & 1\end{array}$

1.1.1 Symptoms, clinical course and diagnosis 1

1.1.2 Pathology of MS 2

$\begin{array}{lll}\text { 1.1.3 Pathogenesis } & 3\end{array}$

1.1.4 The role of astrocytes in the pathogenesis of MS 4

$\begin{array}{ll}\text { 1.1.5 The cuprizone mouse model } & 6\end{array}$

1.2 Regulatory factor X4: Member of the protein family regulatory factor X 7

1.2.1 RFX4_v3: The brain specific variant of RFX4 10

1.2.2 RFX4_v3 deficiency leads to non-communicating congenital hydrocephalus $\quad 10$

1.2.3 Potential target genes and interacting partners of RFX4_v3 11

$\begin{array}{lll}1.3 & \text { Hydrocephalus } & 12\end{array}$

$\begin{array}{lll}\text { 1.3.1 Congenital hydrocephalus } & 13\end{array}$

1.3.2 Subcommissural organ and Reissner fiber glycoproteins 13

$\begin{array}{lll}1.4 & \text { Aims } & 15\end{array}$

$2 \quad$ Materials \& methods $\quad 17$

$\begin{array}{lll}2.1 & \text { Materials } & 17\end{array}$

$\begin{array}{lll}2.1 .1 & \text { Reagents } & 17\end{array}$

$\begin{array}{ll}\text { 2.1.2 Solutions, buffers and cell culture media } & 18\end{array}$

$\begin{array}{lll}2.1 .3 & \text { Enzymes/ proteins } & 20\end{array}$

$\begin{array}{lll}2.1 .4 & \text { Kits } & 21\end{array}$ 
2.1.5 Monoclonal antibodies for flow cytometry

2.1.6 Primary antibodies for immunohistochemistry and fluorescence immunohistochemistry

2.1.7 Secondary antibodies for immunohistochemistry and fluorescence immunohistochemistry

2.1.8 Oligonucleotide primers

2.1.9 TaqMan Expression Assays

2.1.10 Consumables

2.1.11 Technical devices

2.1.12 Software

2.1.13 Tissue from MS patients

\section{$2.2 \quad$ Animals}

\subsection{Mouse strains}

2.3.1 Housing

\section{$2.4 \quad$ Methods}

2.4.1 Genotyping

2.4.2 PCR for the analysis of RFX4 transcript variants in MS

2.4.3 Cuprizone mouse model

2.4.4 Histology

2.4.5 Luxol Fast Blue - Periodic Acid Schiff (LFB-PAS) staining

2.4.6 Hematoxylin and eosin (H\&E) staining

2.4.7 Immunohistochemistry and fluorescence immunohistochemistry

2.4.7.1 Immunohistochemistry

$\begin{array}{lll}\text { 2.4.7.2 } & \text { Fluorescence immunohistochemistry } & 34\end{array}$

2.4.8 Morphometry and data aquisition 34

2.4.9 Isolation of astrocytes from newborn mice 36

2.4.10 In vitro migration assay 36

$\begin{array}{ll}\text { 2.4.11 Quantitative PCR analysis } & 37\end{array}$ 
$\begin{array}{lll}\text { 2.4.11.3 Quantitative PCR } & 38\end{array}$

2.4.12 Isolation of murine microglia cells from the corpus callosum for flow cytometry $\quad 39$

$\begin{array}{lll}2.4 .12 .1 & \text { FACS staining of phagocytes } & 39\end{array}$

$\begin{array}{lll}\text { 2.4.12.2 } & \text { Flow cytometry analysis } & 40\end{array}$

$\begin{array}{lr}\text { 2.4.13 Statistical analysis } & 40\end{array}$

$3 \quad$ Results 41

3.1 Part 1: Characterization of the GFAP cell specific RFX4 conditional knockout mice

3.1.1 Hydrocephalus and mortality of RFX4 conditional knockout mice

3.1.2 Hydrocephalus in RFX4 conditional knockout mice is associated with abnormalities of the subcommissural organ

3.1.3 GFAP cell specific RFX4 conditional knockout mice suffer from congenital hydrocephalus of variable severity

3.1.4 Subcommissural organ hypoplasia $\quad 45$

$\begin{array}{lll}\text { 3.1.4.1 Dysfunction of the SCO } & 46\end{array}$

3.1.5 Loss of developmental factors impairs SCO formation and function 46

3.1.5.1 Characterization of RFX4 deficient P0 astrocytes in vitro $\quad 47$

3.1.5.2 Regulation of hydrocephalus associated genes in RFX4 conditional knockout astrocytes.

3.1.5.2.1 Transcript changes of Wnt signaling

3.1.5.2.2 Expression of factors critical for early brain development and neuronal regulators

\subsection{Part 2: The role of astrocytic RFX4_v3 in demyelination}

3.2.1 RFX4 in MS

3.2.1.1 RFX4 is produced by astrocytes and up-regulated in MS lesions 52

3.2.1.2 RFX4 variant 3 is expressed in MS lesions

3.2.2 Role of astrocytic RFX4_v3 during de- and remyelination in the cuprizone mouse model

3.2.2.1 Cuprizone intoxication for 4 weeks induces strong demyelination in the corpus callosum of RFX4_v3 conditional knockout mice 
3.2.2.1.2 Extensive microglia accumulation in the corpus callosum of RFX4_v3 conditional knockout mice after 4 weeks of cuprizone treatment

3.2.2.1.3 Blood brain barrier is closed in RFX4_v3 deficient mice

3.2.2.1.4 RFX4_v3 deficiency in astrocytes increases the number of microglia in the corpora callosa without differential activation patterns

3.2.2.1.5 Higher numbers of proliferating cells are present in the corpus callosum of RFX4_v3 mutants

3.2.2.1.6 Reduced numbers of oligodendrocytes in the corpus callosum of RFX4_v3 deficient mice after cuprizone exposure

3.2.2.2 RFX4_V3 conditional knockout mice show delayed remyelination

3.2.2.2.1 RFX4_v3 conditional knockout mice remain demyelinated after induction of remyelination for 1 week

3.2.2.2.2 Microglia accumulation in the corpus callosum of RFX4_v3 conditional knockout animals is still increased after 1 week cuprizone withdrawal

3.2.2.2.3 Loss of RFX4_v3 in astrocytes restricts oligodendrocyte differentiation without changing numbers of oligodendrocyte precursor cells

3.2.2.2.4 RFX4_v3 conditional knockout mice show delayed remyelination of the corpus callosum compared to wild type mice

3.2.2.2.5 Numbers of Mac3 positive stained microglia are comparable in the corpus callosum of RFX4_v3 mutants and wild type animals 2 weeks after cuprizone cessation

3.2.2.3 Effects of astrocytic RFX4_v3 inhibition are present after 2 weeks of cuprizone challenge

3.2.2.3.1 RFX4_v3 deficiency markedly accelerates demyelination in vivo

3.2.2.3.2 Astrocyte specific RFX4_v3 deficiency has no influence on the density of activated GFAP positive astrocytes in the corpus callosum.

3.2.2.3.3 Astrocyte specific RFX4_v3 deletion leads to accelerated microglia/ macrophage infiltration into the corpus callosum

3.2.2.4 Deletion of RFX4_v3 in astrocytes increases oligodendrocyte apoptosis and increased mRNA levels of cytokine and chemokines in the corpus callosum

3.2.2.4.1 Increased oligodendroglial apoptosis under loss of RFX4_v3 in astrocytes85

3.2.2.4.2 Inhibition of astrocytic RFX4_v3 substantially increases transcripts for proinflammatory cytokines and chemokines in response to cuprizone treatment in the corpus callosum 


\subsection{Part 1: Characterization of the GFAP cell specific RFX4 conditional}

knockout mice

4.1.1 GFAP specific loss of RFX4 leads to congenital hydrocephalus

4.1.2 Hydrocephalus in RFX4_v3 conditional knockout mice is caused by SCO hypoplasia

4.1.3 Regulation of developmental factors by RFX4_v3 impairs SCO formation and function

4.2 Part 2: The role of astrocytic RFX4_v3 in demyelination

4.2.1 RFX4 is produced by astrocytes and up-regulated in MS lesions

4.2.2 RFX4_v3 expression is important for regulation of myelination and activation of phagocytes in the cuprizone mouse model

4.2.2.1 Increased demyelination in the corpus callosum of RFX4_v3 deficient mice after 4 weeks of cuprizone treatment

4.2.2.2 Remyelination in RFX4_v3 deficient mice is delayed

4.2.2.3 Increased phagocyte activation and accumulation during de- and remyelination

4.2.3 The blood brain barrier is closed in RFX4_v3 deficient mice.

4.2.4 RFX4_v3 deficiency causes microglial cell proliferation without stimulation of microglial activation markers

4.2.5 RFX4_v3 loss in astrocytes modulates chemokine and cytokine transcript levels, thereby attracting phagocytes into the demyelinated corpus callosum

4.2.5.1 Astrocyte specific RFX4_v3 deficiency has no influence on numbers of activated GFAP positive astrocytes in the corpus callosum

4.2.5.2 Inhibition of astrocytic RFX4_v3 substantially increases transcripts of proinflammatory cytokines and chemokines in response to cuprizone 100

4.2.5.3 The transcription factor RFX4_v3 possibly acts as a regulator of cytokine and chemokine production in MS

4.2.6 Deletion of RFX4_v3 in astrocytes induces apoptosis of oligodendrocytes

4.2.6.1 RFX4_V3 may indirectly influence oligodendrocyte apoptosis by changing the inflammatory environment in the corpus callosum

4.2.7 Delayed remyelination as a consequence of a dysregulated inflammatory environment by astrocytes in RFX4_v3 conditional knockout mice

4.2.8 Outlook 
5.1 Part 1: Characterization of the GFAP cell specific RFX4 conditional knockout mice

5.2 Part 2: The role of astrocytic RFX4_V3 in demyelination

References 


\section{Acknowledgements}

First I would like to thank Prof. Dr. Wolfgang Brück for giving me the opportunity to work in his lab. I would like to thank him for the supervision of this project and for scientific advice and discussions.

I would like to offer my special thank to Dr. Andreas Junker for giving me this interresting project. I am thankful for his guidance and constant support over the last years and for sharing his scientific expertise with me.

I also owe a debt of gratitude to Prof. Dr. Christine Stadelmann-Nessler for her constant support, patience and help especially during the last year. I am grateful for her scientific as well as personal advice.

Moreover, I want to thank the members of my thesis committee Prof. Dr. Mikael Simons and Prof. Dr. Hannelore Ehrenreich for the comments and the fruitful discussions and suggestions during my committee meetings. Further thanks to the additional members of my examination board Prof. Dr. Christine Stadelmann, Prof. Dr. Martin Weber and Dr. Francesca Odoardi.

I would like to especially thank Dr. Martina Ott and Dr. Stefan Nessler for scientific discussions, methodological advice and support especially regarding the isolation of murine microglia cells from the corpus callosum for flow cytometry.

I want to thank our technical assistant Uta Scheidt for her daily help and support, for always having an open ear for any kind of problems and especially for having a very good time in the lab.

I also want to acknowledge Brigitte and Katja for her valuable help and support and for having a good time in the lab.

I want to thank my colleagues Verena, Sarah, Anne, Nielsen, Claudia, Darius, Insa, Patrik, Silke and all other colleagues for help and advice and for the pleasant time throughout my thesis.

Further, I want to acknowledge the PhD program Molecular Medicine, especially Dr. Eric Meskauskas and I would like to thank Cynthia and Heidi for the administrative support in the neuropathology. 
I would like to offer my special thanks to Koki, Martina, Alexandra, Pia, Sarah and Thorben for their friendship and for the emotional and constant support. Further, I am grateful to Alexandra and Martina for proofreading of this thesis.

Very personal thanks to my parents Maria and Wolfgang and my siblings Kathrin and Dominik for always supporting me.

Finally, I want to thank my boyfriend Tjebbe for his emotional support, motivation, trust and endless patience. 


\section{Abstract}

\section{Characterization of the GFAP cell specific RFX4 conditional knockout mice}

RFX4_v3 is the brain-specific splice variant of the transcription factor RFX4 (Blackshear et al., 2003; Matsushita et al., 2005). It was shown to play an important role during early brain development and in the genesis of congenital hydrocephalus (Blackshear et al., 2003).

To study the astrocytic role of RFX4_v3 in demyelination, GFAP cell specific RFX4 conditional knockout mice were generated that possess an astrocyte-specific disruption of the RFX4_v3 gene by deletion of exon 4. A proportion of these GFAP cell specific RFX4 conditional knockout mice showed a severe brain phenotype. Macroscopical and histological analysis of the brains at time of birth and during adulthood revealed a hydrocephalus with dramatic extension of the lateral ventricle, which is indicative for a congenital, non-communicating hydrocephalus. Homozygous RFX4 conditional knockout mice were more severely affected than heterozygotes pointing toward a gene dosage effect. The subcommissural organ (SCO), an ependymal brain gland that is important for the patency of the Sylvian aqueduct, was altered and dysfunctional, which was evidenced by reduced or absent SCO glycoprotein (Reissner fiber) secretion. Furthermore, astrocytes from RFX4 conditional knockout mice at the time of birth showed differential expression of genes critical for brain morphogenesis such as Wnt signaling pathways that regulate dorsal midline structuring and development of the SCO. These observations were in line with previous findings (Blackshear et al., 2003) and supported the idea that the hydrocephalus in RFX4 conditional knockout animals is caused by maldevelopment and dysfunction of the SCO. My data further show that RFX4_v3 depletion in GFAP cell lineages including astrocytes and ependymal cells is sufficient to induce congenital hydrocephalus. I conclude that astrocytic and ependymal RFX4_v3 is highly relevant for brain development and the pathogenesis of congenital hydrocephalus.

\section{The role of astrocytic RFX4_v3 in demyelination}

In recent years, growing evidence for a contribution of astrocytes in MS pathogenesis has emerged in the literature. In particular, the immunomodulatory capacities of astrocytes have been suggested to play an important role during de- and remyelination processes in MS (Nair et al., 2008). Recent studies in animal models of MS show that astrocytes influence the activation and recruitment of microglia and peripheral leukocytes by secretion of inflammatory mediators (Skripuletz et al., 2013; Mayo et al., 2014). However, how astrocyte activation and secretion of inflammatory factors, such as 
chemokines and cytokines, is regulated during inflammatory demyelination in MS lesions is poorly understood.

Our group previously identified RFX4_v3 as a highly up-regulated factor in MS lesions. IHC of MS lesions showed RFX4 expression in various cell types with a prominent nuclear localization in astrocytes. The aim of the present study was to investigate the potential astrocytic role of RFX4_v3 in MS and a demyelinating mouse model. Therefore, to study the impact of astrocytic RFX4_v3, GFAP cell specific RFX4_v3 conditional knockout mice were subject to cuprizone induced demyelination. RFX4_v3 deficient mice showed increased demyelination, a reduced number of oligodendrocytes and enhanced axonal damage. In addition, remyelination in RFX4_v3 deficient mice was delayed. These alterations were associated with increased microglia activation in RFX4_v3 deficient mice. Further investigations during the early phase of cuprizone treatment indicated that increased microglia activation may be caused by enhanced oligodendrocyte apoptosis and increased transcript levels of pro-inflammatory chemokines and cytokines in RFX4_v3 deficient mice. This study demonstrates that astrocytic RFX4_v3 expression is important for regulation of myelination and activation of microglia during the processes of de- and remyelination in the cuprizone mouse model. In addition these results point towards a control of the inflammatory environment by the transcription factor RFX4_v3 to restrict the activation and recruitment of microglia cells as well as oligodendrocyte apoptosis, which eventually controls the extent of de- and remyelination in the cuprizone mouse model. Altogether these findings indicate that RFX4_v3 plays a protective role in the cuprizone mouse model. The fact that RFX4_v3 has been described as a transcriptional repressor suggests that astrocytic RFX4_v3 in MS might act as a regulator of production and release of inflammatory mediators and exerts a beneficial role in MS by restricting neuroinflammation. 


\section{List of Figures}

Figure 1: Schematic representation of the six RFX4 isoforms (A) and its RFX family members in mammals (B).

Figure 2: Hydrocephalus and mortality of GFAP cell specific RFX4 conditional knockout mice.

Figure 3: Hydrocephalus of newborn RFX4 conditional knockout mice.

Figure 4: GFAP cell specific RFX4 conditional mice suffer from congenital hydrocephalus of variable severity accompanied by SCO hypoplasia.

Figure 5: Expression of SCO RF glycoprotein is deregulated in RFX4 conditional knockout animals.

Figure 6: Characterization of RFX4 deficient P0 astrocytes in vitro.

Figure 7: Regulation of hydrocephalus associated genes in RFX4 conditional knockout astrocytes.

Figure 8: RFX4 is up-regulated in MS lesions.

Figure 9: RFX4 is expressed by astrocytes and up-regulated in MS.

Figure 10: Only RFX4_v3 is expressed in MS lesions.

Figure 11: Cuprizone feeding for 4 weeks induces strong demyelination in the corpus callosum of RFX4_v3 conditional knockout mice.

Figure 12: Cuprizone-induced demyelination correlates with reduced myelin proteins in the corpus callosum of RFX4 deficient animals.

Figure 13: Increased demyelination was accompanied by enhanced acute axonal damage in the corpus callosum of RFX4_v3 deficient mice.

Figure 14: Extensive accumulation of microglia/macrophages in the corpus callosum of RFX4_v3 conditional knockout mice during demyelination.

Figure 15: Blood brain barrier is closed in RFX4_v3 deficient mice w/o hydrocephalus. 
Figure 16: RFX4_v3 deficiency in astrocytes increases the number of microglia in the corpus callosum after cuprizone feeding without induction of microglial activation markers.

Figure 17: Elevated numbers of proliferating cells in the corpora callosa of RFX4-deficient animals.

Figure 18: Reduced numbers of oligodendrocytes in the corpus callosum of RFX4_v3 deficient mice after cuprizone exposure.

Figure 19: RFX4_v3 conditional knockout mice remain demyelinated after induction of remyelination for 1 week.

Figure 20: RFX4_v3 conditional knockout mice remain demyelinated after induction of remyelination for 1 week.

Figure 21: Microglia accumulation in RFX4_v3 conditional knockout animals is still increased after 7 days cuprizone withdrawal.

Figure 22: Loss of RFX4_v3 in astrocytes restricts oligodendrocyte differentiation without changing numbers of oligodendrocyte precursor cells.

Figure 23: RFX4_v3 conditional knockout mice remyelinate in the corpus callosum with a delay compared to wild type mice.

Figure 24: Numbers of Mac3 positive microglia are comparable in the corpus callosum of RFX4_v3 mutants and wild type animals 2 weeks after of cuprizone cessation. 77

Figure 25: RFX4_v3 deficiency accelerates demyelination in vivo.

Figure 26: Astrocyte-specific RFX4_v3 deficiency has no influence on the density of activated GFAP-positive astrocytes in the corpus callosum.

Figure 27: Astrocyte-specific RFX4_v3 deletion leads to accelerated microglia infiltration into the corpus callosum.

Figure 28: Increased oligodendroglial apoptosis in RFX4_v3 deficient mice.

Figure 29: Loss of RFX4_v3 increases the pro-inflammatory response in the corpus callosum. 
List of tables

Table 1: Reagents

Table 2: $\quad$ Solutions, buffers and cell culture media

Table 3: Enzymes/proteins

Table 4: $\quad$ Kits

Table 5: Monoclonal antibodies for flow cytometry

Table 6: $\quad$ Primary antibodies for immunohistochemistry and fluorescence immunohistochemistry

Table 7: $\quad$ Secondary antibodies for immunohistochemistry and fluorescence immunohistochemistry

Table 8: Oligonucleotide primers

Table 9: TaqMan Expression Assays

Table 10: Consumables

Table 11: Technical devices

Table 12: Software

Table 13: Tissue from MS patients

Table 14: Severity of the hydrocephalus and SCO hypoplasia in RFX4 conditional knockout mice 
Abbreviations

\begin{tabular}{|c|c|}
\hline APP & Amyloid precursor protein \\
\hline AFR & Antiserum against fiber reissner urea \\
\hline ANOVA & Analysis of variance \\
\hline APC & Allophycocyanin \\
\hline APC Cy7 & Allophycocyanin cyanine 7 \\
\hline BBB & blood brain barrier \\
\hline BDNF & Brain derived neurotrophic factor \\
\hline Bmp & Bone morphogenetic protein \\
\hline BSA & Bovine serum albumin \\
\hline BV & Brilliant violet \\
\hline bp & Base pairs \\
\hline c & Caudal \\
\hline cDNA & Complementary deoxyribonucleic acid \\
\hline CCL2 & Chemokine (C-C motif) ligand 2 \\
\hline CCL3 & Chemokine (C-C motif) ligand 3 \\
\hline CCR5 & Chemokine (CC motif) receptor 5 \\
\hline CXCL12 & C-X-C motif chemokine 12 \\
\hline CXCR3 & Chemokine (CXC motif) receptor 3 \\
\hline CXCL10 & Chemokine (C-X-C motif) ligand 10 \\
\hline CX3CL1 & Chemokine (CX3C motif) ligand 1 \\
\hline CX3CR1 & Chemokine (CX3C motif) receptor 1 \\
\hline CD & Cluster of differentiation \\
\hline CNS & Central nervous system \\
\hline CNPase & 2',3'-Cyclic-nucleotide 3'-phosphodiesterase \\
\hline CNTF & Ciliary neurotrophic factor \\
\hline CSF & Cerebrospinal fluid \\
\hline CT & Cycle treshold \\
\hline Ctnnb1 & Beta-catenin-1 \\
\hline Cyp26a & Cytochrome P450 26A1 \\
\hline Cy2 & Cyanine 2 \\
\hline Cy3 & Cyanine 3 \\
\hline DAB & 3,3'-Diaminobenzidine \\
\hline DAPI & 4',6-Diamidin-2-phenylindole \\
\hline
\end{tabular}




\begin{tabular}{|c|c|}
\hline DBD & DNA binding domain \\
\hline DNA & Deoxyribonucleic acid \\
\hline DNase & Desoxyribonuclease \\
\hline dNTP & Desoxynucleoside triphosphate \\
\hline $\mathbf{E}$ & Embryonic stage \\
\hline EDTA & Ethylenediamine tetraacetic acid disodiumsalt dihydrate \\
\hline EAE & Experimental autoimmune encephalomyelitis \\
\hline En-1 & Engrailed-1 \\
\hline FACS & Fluorescence-activated cell sorting \\
\hline FCS & Fetal calf serum \\
\hline FITC & Fluorescein isothiocyanate \\
\hline Foxa2 & Forkhead box $A 2$ \\
\hline Fzd10 & Frizzled class receptor 10 \\
\hline FRU & Fiber reissner urea \\
\hline GFAP & Glial fibrillary acidic protein \\
\hline GapDH & Glyceraldehyde-3-phosphate dehydrogenase \\
\hline GM-CSF & Granulocyte macrophage colony-stimulating factor \\
\hline HBSS & Hank's buffered salt solution \\
\hline H\&E & Hematoxylin and eosin \\
\hline ICAM-1 & Intercellular adhesion molecule 1 \\
\hline IFN & Interferon \\
\hline IGF1 & Insulin-like growth factor 1 \\
\hline IgG & Immunoglobulin G \\
\hline IHC & Immunohistochemistry \\
\hline IL-1ß & Interleukin 1 beta \\
\hline Ki & Kiel \\
\hline LFB-PAS & Luxol Fast Blue Periodic Acid Schiff \\
\hline Lv & Lateral ventricle \\
\hline LacCer & Lactosylceramide \\
\hline L1CAM & L1 cell adhesion molecule \\
\hline LFA-1 & Lymphocyte function-associated antigen 1 \\
\hline LPS & Lipopolysaccharide \\
\hline LIF & Leukemia inhibitory factor \\
\hline $\mathrm{mM}$ & Millimolar \\
\hline
\end{tabular}




\begin{tabular}{|c|c|}
\hline mm & Millimeter \\
\hline Mac & Macrophage \\
\hline MACS & Magnetic-activated cell sorting \\
\hline MAG & Myelin-associated glycoprotein \\
\hline MBP & Myelin basic protein \\
\hline $\mathrm{MHCl}$ & Major histocompatibility complex class I molecule \\
\hline MHCII & Major histocompatibility complex class II molecule \\
\hline $\min$ & Minute(s) \\
\hline ml & Milliliter \\
\hline NAWM & Normal appearing white matter \\
\hline$\mu \mathrm{g}$ & Microgram \\
\hline$\mu l$ & Microliter \\
\hline$\mu \mathrm{m}$ & Micrometer \\
\hline$\mu \mathrm{M}$ & Micromolar \\
\hline MOG & Myelin oligodendrocyte glycoprotein \\
\hline MRI & Magnetic resonance imaging \\
\hline MRP14 & Myeloid-related protein 14 \\
\hline mRNA & Messenger ribonucleic acid \\
\hline Msx3 & Msh homeobox 3 \\
\hline MS & Multiple sclerosis \\
\hline $\mathbf{n}$ & Number of independent samples or animals \\
\hline NAWM & Normal appearing white matter \\
\hline ng & Nanogram \\
\hline $\mathrm{nm}$ & Nanometer \\
\hline Olig & Oligodendrocyte transcription factor \\
\hline OPC & Oligodendrocyte precursors cells \\
\hline PFA & Paraformaldehyde \\
\hline PBS & Phosphate buffered salt solution \\
\hline P25 & Brain-specific 25-kDa protein \\
\hline PLP & Proteolipid protein \\
\hline PPMS & Primary progressive \\
\hline $\mathbf{P}$ & Proline \\
\hline $\mathbf{P}$ & Postnatal stage \\
\hline Pax & Paired box \\
\hline
\end{tabular}




\begin{tabular}{|c|c|}
\hline PCR & Polymerase chain reaction \\
\hline PC & Posterior commissure \\
\hline PE & Phycoerythrin \\
\hline PerCP & Peridinin chlorophyll protein \\
\hline Q & Glutamine-rich \\
\hline qPCR & Quantitative polymerase chain reaction \\
\hline RA & Retinoic acid \\
\hline RFX & Regulatory factor $X$ \\
\hline RFX4_v & Regulatory factor X 4_variant \\
\hline Rspo1 & R-spondin 1 \\
\hline $\mathbf{r}$ & Rostral \\
\hline RF & Reissner fiber \\
\hline RNA & Ribonucleic acid \\
\hline RT PCR & Real time polymerase chain reaction \\
\hline S.aq & Sylvian aqueduct \\
\hline SEM & Standard error of the mean \\
\hline sco & Subcommissural organ \\
\hline SPMS & Secondary progressive MS \\
\hline TGF-ß & Transforming growth factor beta \\
\hline TNF- $\alpha$ & Tumor necrosis factor alpha \\
\hline TNFR & Tumor necrosis factor receptor \\
\hline TH1 & T helper 1 \\
\hline TH17 & T helper 17 \\
\hline Tim-3 & T-cell immunoglobulin domain and mucin domain 3 \\
\hline TLR & Toll-like receptors \\
\hline Trem2b & Triggering Receptor Expressed on Monocytes 2 \\
\hline IIIv & Third ventricle \\
\hline VCAM-1 & Vascular cell adhesion molecule 1 \\
\hline VLA-4 & Very Late Antigen-4 \\
\hline vtn & Vitronectin \\
\hline Wnt3 & Wingless-type MMTV integration site family, member 3 \\
\hline Wnt3a & Wingless-type MMTV integration site family, member $3 a$ \\
\hline
\end{tabular}




\section{Introduction}

\section{$1.1 \quad$ Multiple sclerosis}

Multiple sclerosis (Encephalomyelitis disseminata) is a chronic inflammatory demyelinating disease of the central nervous system (CNS), which was first described in detail by the French neurologist Jean Martin Charcot in 1868. The etiology of multiple sclerosis (MS) is not fully clarified, but it is widely believed that MS is a T cell mediated autoimmune disorder of the CNS. MS affects approximately 2.5 million people worldwide and is most often diagnosed between the early and middle adulthood. The disease leads to lesions, also known as plaques, in the white and gray matter of the CNS. These lesions are characterized by demyelination, inflammation, axonal loss and gliosis (Brück, 2005; Milo and Kahana, 2010; Lassmann, 2011; Kutzelnigg and Lassmann, 2014). Astrocytes are the most abundant cell type in MS lesions and, for a long time, they were considered to only play a secondary role in MS. However, more recently it was shown that astrocytes play an active role in MS through their immunomodulatory capacities (Williams et al., 2007; Nair et al., 2008; Brosnan and Raine, 2013).

\subsubsection{Symptoms, clinical course and diagnosis}

In MS, symptoms and clinical course of individual patients cover a broad spectrum. Common symptoms are blurred or double vision for several days to weeks, loss of balance, fatigue, numbness and tingling, weakness in arms or legs and hemiparesis. These symptoms wax and wane over time.

Patients with MS typically display one of the following clinical courses: Relapsing remitting (RR), secondary progressive (SP) or primary progressive (PP) (Thompson, 2004; Lublin et al., 2014).

Relapsing remitting multiple sclerosis (RRMS) is a disease form where patients have relapses and periods of recovery or remission in between relapses. RRMS is the most frequent initial form of MS with about $80-85 \%$ of MS patients being affected. The relapse period is characterized by appearance of new or worsening symptoms for a period of at least 24 hours or longer. The relapse period is followed by a period of recovery or remission in which symptoms partially or completely disappear.

Most patients with RRMS later develop secondary progressive MS (SPMS). SPMS is defined by a switch from relapses and remission to a steady and chronic deterioration.

About $10-20 \%$ of the patients are initially diagnosed with primary progressive MS (PPMS) that shows no relapses and remission. In general, PPMS is diagnosed later than other types of MS. Due to a greater degree of spinal cord abnormalities, patients with 
PPMS often show spinal cord symptoms such as weakness in arms or legs, fatigue and bladder and bowel difficulties (Thompson, 2004).

MS is diagnosed by the Mc Donald criteria, which were introduced 2001 with the latest revision in 2011 (McDonald et al., 2001; Polman et al., 2011; Lublin et al., 2014). These diagnosis criteria include analysis of clinical symptoms, cerebrospinal fluid parameters and MRI data, which allows the diagnosis of MS after a single symptomatic episode (Polman et al., 2011).

\subsubsection{Pathology of MS}

The pathological hallmark of MS is the presence of multifocal lesions in the gray and white matter of the CNS. These lesions are characterized by an inflammatory infiltrate, axonal loss, astrogliosis, extensive demyelination and a variable degree of remyelination (Brück and Stadelmann, 2005). The MS plaques are present throughout the CNS with preferential localization in the subcortical and periventricular white matter, the spinal cord, optic nerve and the pons and medulla (Kutzelnigg and Lassmann, 2014). Macroscopically, focal demyelinating lesions of the white matter appear as gray to brownish discolored areas, with a size between less than a millimeter up to several centimeters (Brück and Stadelmann, 2005; Kutzelnigg and Lassmann, 2014).

Based on the demyelinating activity MS lesions can be divided into active lesions characterized by an extensive inflammatory infiltrate and ongoing demyelinating activity and inactive lesions that are hypocellular and show no evidence of myelin breakdown (Pittock and Lucchinetti, 2007).

Active lesions are subdivided in early and late active lesions. Early active lesions contain macrophages and activated microglia containing myelin debris reactive for myelin oligodendrocyte glycoprotein (MOG), myelin associated glycoprotein (MAG), myelin basic protein (MBP), proteolipid protein (PLP) and 2',3'-Cyclic-nucleotide 3'-phosphodiesterase (CNPase) and express the early activation marker myeloid-related protein 14 (MRP14). In contrast, late active lesions contain macrophages and microglia with myelin debris, which are only reactive for MBP and PLP - an indication for advanced myelin degradation. Late active lesions loose MRP14 expression (Brück et al., 1995; Lucchinetti et al., 2000).

Inactive lesions are characterized by fibrillary gliosis, reduced axonal density, diminished or absent mature oligodendrocytes and a variable degree of inflammation. However, phagocytes in inactive lesions do not contain myelin debris that are immunoreactive for any of the myelin proteins described for active MS lesions.

MS lesions with ongoing remyelination, a CNS intrinsic repair mechanism, are divided into early and late remyelinating lesions. Early remyelinating lesions contain a phagocytic inflammatory infiltrate and axons surrounded by thin myelin sheaths, while late 
remyelinating lesions (shadow plaques) contain no foamy phagocytes, axons surrounded by newly formed thin myelin sheath and mild astrogliosis (Brück et al., 1995).

\subsubsection{Pathogenesis}

MS is a chronic inflammatory demyelinating disease of the CNS. It is believed that infiltration of autoreactive T cells into the CNS is the primary trigger and cause of demyelination in MS. The inflammatory infiltrate in lesions mainly contains $\mathrm{CD} 4^{+}$and $\mathrm{CD}^{+} \mathrm{T}$ lymphocytes, activated phagocytes, few B lymphocytes and plasma cells. This inflammatory reaction in MS is accompanied by up-regluation of cytokines (e.g. interleukin 2 (IL-2), IL-1, IL-12, tumor necrosis factor alpha (TNF- $\alpha$ )) and chemokines (e.g. chemokine ( $\mathrm{C}-\mathrm{C}$ motif) ligand 5 (CCL5), CCL3, chemokine (C-X-C motif) ligand 10 (CXCL10)) including their receptors (e.g. chemokine (CC motif) receptor 5 (CCR5), chemokine (CX3C motif) receptor 1 (CX3CR1)), which are associated mainly with Th1 mediated processes. Further it has been demonstrated by MRI studies of MS patients that many lesions are preceded by focal blood brain barrier (BBB) breakdown (Lucchinetti et al., 1998; Lucchinetti et al., 2001; Pittock and Lucchinetti, 2007). In addition, activated endothelial cells in active lesions express adhesion molecules, fibronectin, major histocompatibility complex class II (MHC class II) molecules and stress molecules, which were shown to impact on the BBB integrity (Lucchinetti et al., 2000; Minagar and Alexander, 2003). These observations are highly similar to experimental autoimmune encephalomyelitis (EAE), a T-cell mediated autoimmune disease and animal model for human MS. EAE is induced by either administration of myelin proteins or peptides in adjuvants or by the adoptive transfer of autoreactive T cells. Based on the MS pathology and EAE it is believed that $T$ cells become activated in the periphery, attach to the vessel endothelium by interacting with adhesion molecules and pass through the BBB to enter the CNS. In the CNS these T cells are reactivated by antigen presenting cells and produce pro-inflammatory cytokines and chemokines, thereby recruiting phagocytes and increasing the permeability of the BBB. This leads to an increasing inflammatory infiltrate and subsequent myelin destruction (Baker et al., 1991; Lucchinetti et al., 1998; Lucchinetti et al., 2001; Pittock and Lucchinetti, 2007; Williams et al., 2007).

Although MS is mainly considered as a T cell mediated disease, innate immune cells such as microglia/macrophages and dendritic cells contribute to disease pathogenesis (Gandhi et al., 2010). In MS patients, dendritic cells are activated, show increased expression of activation markers and secrete pro-inflammatory cytokines to promote $\mathrm{T}$ cell responses (Karni et al., 2006). Microglia/macrophages contribute to MS through the secretion of pro-inflammatory molecules and antigen presentation, thereby promoting 
inflammation. In addition, these cells are involved in demyelination and phagocytosis of the degraded myelin (Bauer et al., 1994; Benveniste, 1997).

Over the last years, it became clear that astrocytes also contribute to MS pathogenesis. Astrocytes were believed to only play a role in providing a support mechanism for neuronal functions such as regulating the local ion and $\mathrm{pH}$ homeostasis, storing CNS glycogen, uptaking of glutamate and clearing neuronal waste (Kang et al., 1998; Nedergaard et al., 2003; Liberto et al., 2004). However, astrocytes actually play an active role in MS through their immunomodulatory capacity (Williams et al., 2007; Nair et al., 2008; Brosnan and Raine, 2013).

\subsubsection{The role of astrocytes in the pathogenesis of MS}

In response to tissue damage astrocytes become reactive and undergo astrogliosis. Astrogliosis is characterized by hypertrophy of cellular processes, proliferation and the up-regulation of type III intermediate filament proteins such as the glial fibrillary acidic protein (GFAP) and vimentin, which form an intermediate filament network. In MS reactive and proliferative astrocytes can form a glia scar, a dense network of hypertrophic cells and processes. These cellular processes are accompanied by changes in gene expression of growth factors, cytokines, chemokines, receptors and adhesion molecules. Accordingly, astrocytes exhibit multiple immune functions that are involved in MS pathogenesis (Williams et al., 2007; Sofroniew and Vinters, 2010; Sofroniew, 2014).

One major function of astrocytes is maintaining the BBB, which is a selective permeable barrier that separates the circulating blood from the CNS parenchyma. The BBB is composed of endothelial cells, basal lamina, perivascular pericytes and astrocyte endfeets. By production and secretion of chemokines and cytokines astrocytes can modulate the BBB permeability. The pro-inflammatory factors IL-6 and IL- $1 ß$ were reported to increase BBB permeability during inflammation, whereas transforming growth factor beta (TGF-ß) tightens the BBB (Schwaninger et al., 1999; Didier et al., 2003).

In addition, astrocytes activate autoreactive $T$ cells by expression of adhesion molecules, in particular intercellular adhesion molecule 1 (ICAM-1) and vascular cell adhesion molecule 1 (VCAM-1). ICAM-1 was detected in astrocytes of demyelinating MS lesions (Cannella and Raine, 1995), while VCAM-1 was identified in astrocytes during EAE (Sobel et al., 1990; Gimenez, Mary Ann T et al., 2004). These adhesion molecules bind to LFA-1 and VLA-4 on activated lymphocytes, an important step during migration of $\mathrm{T}$ cells through the vascular endothelium into the parenchyma (Gimenez, Mary Ann T et al., 2004).

Another way in which astrocytes may promote MS pathogenesis is through acting as non-professional antigen presenting cells. Although the literature is controversial there is evidence that in human MS tissue astrocytes express MHC class I and class II and 
costimulatory molecules such as CD80 and CD86, which may promote the activation of autoreactive T cells (Zeinstra et al., 2000; Zeinstra et al., 2003).

Furthermore, astrocytes express Toll-like receptors (TLR), a class of innate immune receptors that are critical in modelating cytokine and chemokine secretion in response to exogenous pathogens. In MS astrocytic TLR3 expression has been observed in late stage MS lesions (Bsibsi et al., 2002). On the one side, TLR3 stimulation on astrocytes triggers the release of pro-inflammatory cytokines such as type I interferons (IFN- $\alpha, \beta)$ and IL-6, which in turn cause an increase in MHC class I and class II expression and thus promote a pro-inflammatory response (Carpentier et al., 2007). On the other side, TLR3 activation on astrocytes was shown to trigger the release of neuroprotective factors such as brain-derived neurotrophic factor (BDNF) and ciliary neurotrophic factor (CNTF) and anti-inflammatory cytokines (IL-9, IL-10, IL-11) to inhibit, for example, the secretion of IL12 and IL-23 (Cua et al., 2003; Jack et al., 2005; Bsibsi et al., 2006). The secretion of pro- and anti-inflammatory mediators as well as neurotrophic factors has both detrimental and beneficial consequences for de- and remyelination in MS lesions.

The astrocytic secretion of pro-inflammatory cytokines and chemokines recruits $T$ cells, macrophages and microglia to the demyelinating lesion, which supports demyelination. Based on EAE and in vitro studies astrocyte-derived cytokines such as IL12, IL-17, IL-23 and TNF- $\alpha$ lead to T helper 1 (TH1) and T helper 17 (TH17) cell response (Langrish et al., 2005; McFarland and Martin, 2007; Miljkovic et al., 2007). Moreover, astrocytes were shown to be major producers of the chemokines CCL5, CXCL12, CCL2, CXCL8, and CXCL10, which recruit macrophages, microglia, and lymphocytes towards MS lesions or have effects on migration of lymphocytes across the BBB (Dong and Benveniste, 2001; Calderon et al., 2006; Mayo et al., 2014).

By contrast, astrocytes also can create an environment that inhibits demyelination and supports remyelination. Astrocytes expressing anti-inflammatory molecules such as IL10, TGF-ß, IL-4 and IL-5 lead to an inhibition of the Th1 and Th17 response (Bjartmar and Trapp, 2001; Bsibsi et al., 2006). By secretion of chemokines and neurotrophic factors astrocytes promote oligodendrocyte precursor cells (OPC) migration (CCL2), proliferation (IL-6, IL-11, insulin-like growth factor 1 (IGF-I)), maturation (leukemia inhibitory factor (LIF), IL-11) and survival (BDNF, CNTF) (Barres et al., 1993; Gensert and Goldman, 1997; Bjartmar and Trapp, 2001). 


\subsubsection{The cuprizone mouse model}

To study MS pathology and pathogenesis several animal models have been established in the past. Each animal model only mimics parts of MS pathology. The most widely used model is EAE in mice. In EAE, immunization with myelin antigens or adoptive transfer of myelin specific $\mathrm{T}$ lymphocytes induces inflammation, demyelination, axonal loss and gliosis in the CNS, with a preference for the spinal cord (Torkildsen et al., 2008; Croxford et al., 2011). This model is mainly used to study the inflammatory aspects of MS.

The cuprizone mouse model was established to study the process of demyelination and remyelination. In contrast to $E A E$, the $B B B$ is intact in this model, which allows to study de- and remyelination without significant influence of the peripheral immune system (Kondo et al., 1987; Baker et al., 1991; McMahon et al., 2002). Cuprizone (biscyclohexanone-oxaldihydrazone) is a copper chelating reagent, which is typically added to normal diet at a concentration of $0.2-0.25 \%$. Mice are then fed for 4 to 6 weeks to induce significant and reproducible demyelination. In young adult mice cuprizone diet directly or indirectly causes oligodendrocyte apoptosis (after 3 to 7 days) with subsequent demyelination of several brain areas including corpus callosum, hippocampus, anterior commissure, olfactory bulb, optic chiasm, brain stem, cerebellum, caudate, putamen, cerebral cortex and cingulum (Matsushima and Morell, 2001; Gudi et al., 2009; Koutsoudaki et al., 2009; Hesse et al., 2010; Silvestroff et al., 2010; Gudi et al., 2014). The mechanism of cuprizone-induced oligodendrocyte damage is not completely understood. However, it is believed that selective toxicity of cuprizone towards oligodendrocytes is mediated by mitochondrial dysfunction leading to oligodendrocyte death and initiation of demyelination (Matsushima and Morell, 2001; Pasquini et al., 2007; Ransohoff, 2012; Bénardais et al., 2013). Early loss of oligodendrocytes results in astrogliosis, microglial activation, and demyelination in the following weeks of cuprizone diet (Hiremath et al., 1998). Microglia cells become activated in week 1 and significantly increase after week 3 to clear myelin debris (Hiremath et al., 1998). Once demyelination is complete microglia reach their maximum density and start to decrease (Mason et al., 2001a). The most abundant phagocyte population in the cuprizone mouse model is the CD11b-positive and CD45-low population, which identifies parenchymal microglia (Remington et al., 2007; Voss et al., 2012). Only 0.5-7\% are infiltrating macrophages (Remington et al., 2007; Voss et al., 2012). In addition, the group of Trevor Owens found an increase of CD86 and MHC class I receptors on microglia cells in cuprizone-treated mice. Hereby, the mean fluorescent intensity of CD80 only increased slightly and expression of MHC class II and CD40 was unchanged (Remington et al., 2007). In vitro, these cells revealed high phagocytic activity and up-regulated phagocytic receptors, 
including Trem2b, CD23b (FcgRII/III), CD36 and Tim-3 (Voss et al., 2012). These results indicate that phagocytosis is the dominant characteristic of microglia cells in the cuprizone mouse model (Remington et al., 2007; Voss et al., 2012).

During the first 2 weeks of cuprizone treatment astrocytes become hypertrophic with thick processes and up-regulate GFAP expression. Astrocytes significantly increase in number after week 3 , when significant levels of demyelination are present (Hiremath et al., 1998). This phase of acute demyelination is followed by spontaneous remyelination upon cuprizone withdrawal. Thereby, extensive remyelination is evident after 3-4 weeks (Matsushima and Morell, 2001; Mason et al., 2004; Lindner et al., 2008; Torkildsen et al., 2008).

In the cuprizone mouse model astrocytes and microglia are thought to express and secrete cytokines and chemokines, which are important for microglia activation and recruitment to clear myelin debris, and to stimulate migration, proliferation and regeneration of myelin forming oligodendrocytes (Arnett et al., 2001; Kotter et al., 2006; Raasch et al., 2011; Skripuletz et al., 2013). The chemokine CCL2 is strongly upregulated during the first weeks in the cuprizone mouse model (McMahon et al., 2001). CCL2 is produced by astrocytes and is relevant for microglia migration and recruitment (Ransohoff et al., 1993; Hayashi et al., 1995; Raasch et al., 2011; Kim et al., 2014).

Deletion of the chemokine CCL3 in the cuprizone mouse model delayed demyelination, which was accompanied by reduced recruitment of microglia and astrocytes (McMahon et al., 2001). The work from Skripuletz and colleagues implicated that also astrocytic CXCL10 is crucial for activation and recruitment of microglia and macrophages (Skripuletz et al., 2013). In the cuprizone mouse model the cytokines IL-1ß and TNF- $\alpha$ infere with oligodendrocyte recruitment, proliferation and differentiation. (Lee et al., 1993; Mason et al., 2001b; Raasch et al., 2011). TNF- $\alpha$ acts through two receptors TNFR1 and TNFR2, whereas in the cuprizone mouse model TNF- $\alpha$ only acts through TNFR2, which is critical for oligodendrocyte regeneration (Arnett et al., 2001). Accordingly, TNF- $\alpha$ and IL-1ß deletion in the cuprizone mouse model significantly reduces remyelination (Arnett et al., 2001; Mason et al., 2001b).

\subsection{Regulatory factor X4: Member of the protein family regulatory factor $X$}

In 2008, Cahoy and colleagues provided a detailed characterization and comparison of genes expressed by isolated astrocytes, neurons, and oligodendrocytes from the developing and mature mouse forebrain (Cahoy et al., 2008). In this study RFX4 was found to be highly enriched in astrocytes. Based on this work Dr. Andreas Junker performed a quantitative PCR study of 96 astrocyte specific genes of MS lesions together 
with normal brain white matter as a control to determine astrocyte functions during inflammatory demyelination. In this study he found RFX4 to be highly up-regulated in MS lesions. In the present work the impact of the transcription factor RFX4 for inflammatory demyelination was further investigated in the cuprizone mouse model.

In 1992, Dotzlaw and colleagues isolated human RFX4 from breast cancer as a partial cDNA, which encoded a short RFX-type DNA-binding domain (DBD) fused to the estrogen receptor (Dotzlaw et al., 1992). RFX4 is a member of the protein family regulatory factor $X(R F X)$, which belongs to the winged-helix subfamily of helix-turn-helix transcription factors (Gajiwala et al., 2000). This protein family shares a highly conserved 76-amino-acid winged helix DNA binding domain and regulates the expression of their target genes by binding to the X-box consensus sequence (Gajiwala et al., 2000). These transcription factors are present in many eukaryotic organisms such as nematodes, fungi, yeast, fruit flies and mammals (Morotomi-Yano et al., 2002).

Five RFX proteins (RFX1-RFX5) have been identified in mammals (Morotomi-Yano et al., 2002). Besides the conserved DBD, RFX4 contains 3 additional conserved regions including region $B, C$ and the dimerization domain, which are highly conserved in RFX1, RFX2 and RFX3 (Figure 1). Compared to other members of the RFX family RFX4 lacks the $\mathrm{Q}$ (glutamine-rich), the $\mathrm{PQ}$ (proline and glutamine-rich) and the A region (Figure 1). These regions are important for transcriptional activation. Therefore, it has been suggested that RFX4 is rather a transcriptional repressor than a transcriptional activator (Morotomi-Yano et al., 2002). However, Zhang and colleagues showed that RFX4 can also act as transcriptional activator as shown for activation of chemokine (CX3C motif) ligand 1 (CX3CL1) (Zhang et al., 2006a).

RFX transcription factors, such as RFX2, RFX3 and RFX4 have been described to play a crucial role in regulation of ciliogenesis by controlling many genes involved in cilia assembly or function (Swoboda et al., 2000; El Zein et al., 2009; Thomas et al., 2010; Didon et al., 2013; Kwon et al., 2014). RFX3 deficient mice show several features of ciliopathies, which includes left-right asymmetry defects and hydrocephalus (Didon et al., 2013). In addition, RFX3 regulates early midline structuring and is required for corpus callosum development (Benadiba et al., 2012). RFX4 itself has been shown to regulate sonic hedgehog signaling to control ciliogenesis in certain regions (Ashique et al., 2009).

Members of the RFX family are implicated in regulation of genes important for the immune response. RFX1 is a well characterized member, which modulates the expression of interleukin-5 receptor a (Gajiwala et al., 2000). In addition, a recent paper suggests that RFX1 directly regulates CD44 expression, which may contribute to proliferation, survival and invasion of glioblastoma cells (Feng et al., 2014). The best 
characterized member of the RFX family is RFX5, which is structurally different from all other human RFX members only sharing the DNA binding domain with other members of the RFX family (Steimle et al., 1995; Emery et al., 1996). RFX5 binds to the MHC class II promoter and is essential for the MHC class II gene expression (Moreno et al., 1997). Germline mutations of the RFX5 gene lead to the bare lymphocyte syndrome, a disease with severe immunodeficiency (Steimle et al., 1995; Reith and Mach, 2001). In addition, CX3CL1, a chemokine gene highly expressed in the brain was identified as direct target for RFX4_v3 (Tarozzo et al., 2003; Zhang et al., 2006a).

Up to now six alternative splice variants of RFX4 have been identified. These six isoforms are either termed as RFX4_v1, _v2, _v3, _v4, _v5, _v6 or RFX4 A, -B, -C, -D, -E, -F (Figure 1). RFX4_v1 and RFX4_v2 were the first isolated isoforms of RFX4 (MorotomiYano et al., 2002). These two isoforms together with variant 4 are testis specific (Blackshear et al., 2003; Matsushita et al., 2005). The study from Matsushita and colleagues showed that variant 3 is the only variant expressed in normal brain tissue. The mRNAs of RFX4 variant 5 and 6 in addition to the mRNA of RFX4_v3 were found in gliomas, but no expression was observed in other tumors such as lung, esophageal, stomach, colon or liver cancers (Matsushita et al., 2005). RFX4 variant 5 and 6 lack the DBD (Figure 1). Therefore, it is unknown whether they can still act as transcription factors.

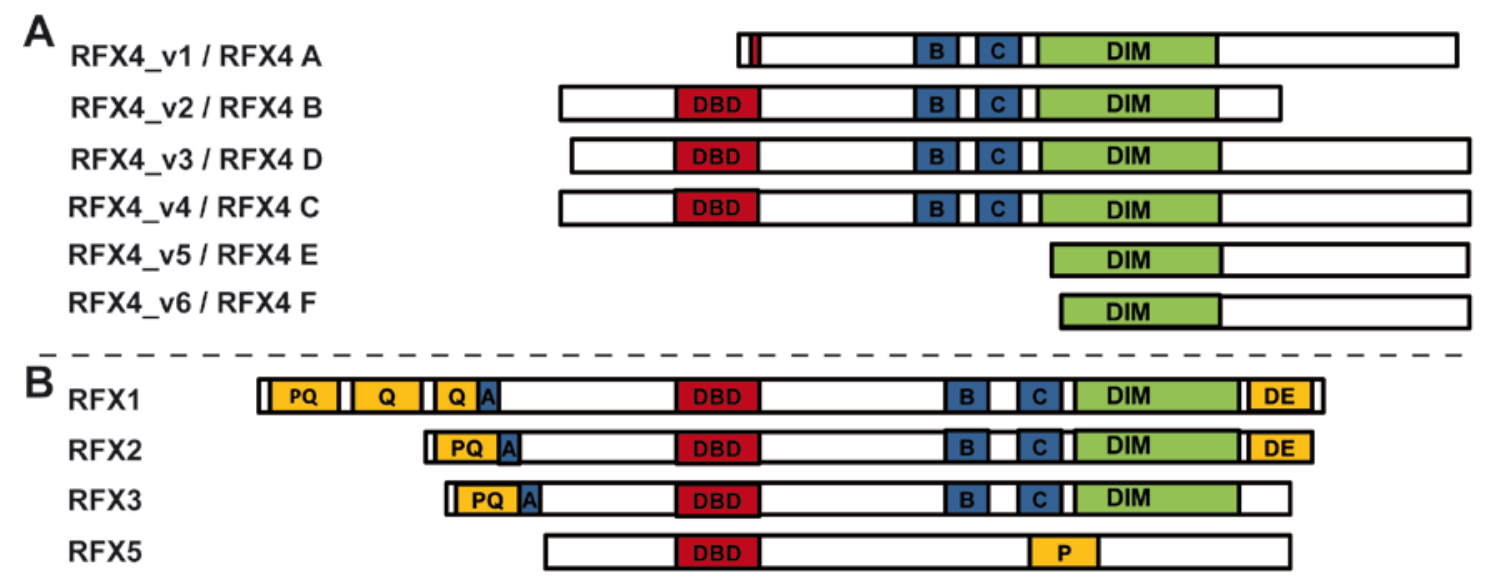

Figure 1: Schematic representation of the six RFX4 isoforms (A) and its RFX family members in mammals (B).

$\mathrm{A}, \mathrm{B}$, and $\mathrm{C}$ represent evolutionarily conserved regions, $\mathrm{DE}=$ acidic region, $\mathrm{P}=$ proline-rich region, $\mathrm{Q}$ = glutamine-rich regions, $\mathrm{PQ}=$ proline and glutamine rich region, $\mathrm{DBD}=\mathrm{RFX}$-type DNA-binding domain, DIM = dimerization domain. 


\subsubsection{RFX4_v3: The brain specific variant of RFX4}

In 2003, Blackshear and colleagues generated transgenic mice for cardiac specific expression of the human epoxygenase gene (Blackshear et al., 2003). One line of these strains showed a brain phenotype including head swelling and rapid neurological decline in young adulthood (Blackshear et al., 2003). Analysis of the genomic sequences revealed that the transgene was inserted into an intron of the mouse RFX4 locus. This insertion created a null allele for the expression of the RFX4 variant 3 (Blackshear et al., 2003). An alignment of the human and mouse RFX4_v3 amino acid sequences showed 96\% sequence identity between the mouse and human RFX4_v3 protein (Blackshear et al., 2003), which could point towards similar functions of RFX4_v3 in mouse and human.

The RFX4_v3 expression is restricted to the brain. RFX4_v3 expression is found during early development as well as during adulthood (Blackshear et al., 2003; Matsushita et al., 2005). The first expression of RFX4_v3 was detected at embryonic stage (E) 8.5 in the neural plate. At E9.5 RFX4_v3 is expressed in two large regions, the spinal cord and the caudal diencephalon/mesencephalon, while at E10.5 the expression extends throughout the neural tube. From E12.5 to birth, levels of RFX4_v3 mRNA were detected in the neuroepithelium and the ependym of the neural tube. In addition, from E14.5 to birth RFX4_v3 transcripts were found in the ependymocytes of the developing subcommissural organ (SCO) (Blackshear et al., 2003). In the adult brain RFX4_v3 is expressed in several brain tissues including brainstem, midbrain, cerebellum, hippocampus, striatum, frontal lobe and olfactory bulb (Zhang et al., 2007). In addition, Araki and colleagues found high levels of RFX4_v3 in the suprachiasmatic nucleus. This implicated that RFX4 function may be related to the circadian clock, because the suprachiasmatic nucleus is the central clock locus for the circadian rhythm (Araki et al., 2004).

\subsubsection{RFX4_v3 deficiency leads to non-communicating congenital hydrocephalus}

As mentioned before, RFX4_v3 deficient mice develop head swelling and neurological decline in young adulthood. Investigations of these transgenic mice line revealed severe hydrocephalus in all mice at the time of birth, indicative of a congenital hydrocephalus (Blackshear et al., 2003). Disruption of both RFX4_v3 alleles leads to severe brain deformation with failure of dorsal midline formation. All pups homozygous for RFX4_v3 deletion died shortly before or after birth (Blackshear et al., 2003). RFX4_v3 +/- mice survived, but suffered from congenital hydrocephalus associated with hypoplasia or absence of the SCO. 
Histological analysis of the brains showed severe hydrocephalus in the anterior brain, with dramatic extension of the lateral ventricles. The SCO was absent in the transgenic mice, while other midline structures such as the pineal body and posterior commissure appeared to be normal (Blackshear et al., 2003). Furthermore immunohistochemistry with an antibody specific for SCO-produced Reissner's fibers showed in contrast to wild type mice an absence of SCO glycoproteins in RFX4_v3 +/- mice (Blackshear et al., 2003). Therefore, the agenesis and resulting dysfunction of the SCO was considered as the main cause for the hydrocephalus in RFX4_v3 deficient mice (Blackshear et al., 2003; Zhang et al., 2006a).

Interruption of both alleles of RFX4_v3 severely alters brain morphogenesis (Blackshear et al., 2003). Heads of RFX4_v3 -/- mice at E12.5 are smaller than of wild type mice and show an abnormal doming of the skull. Heads of RFX4_v3 -/- lack morphological differentiation of dorsal structures, which was most obvious by the loss of dorsal midline structures and the formation of a single central ventricle (Blackshear et al., 2003). Blackshear and colleagues assumed that disruption of both alleles possibly interferes with the expression of downstream genes, which leads to failed dorsal midline structuring (Blackshear et al., 2003; Zhang et al., 2006a).

\subsubsection{Potential target genes and interacting partners of RFX4_v3}

To identify potential target genes of RFX4_v3 the group of Blackshear and colleagues performed a microarray analysis of RFX4_v3 +/+ and RFX4_v3 -/- mouse brains at the embryonical stage 10.5 to identify differentially expressed genes in RFX4_v3 -/- mice (Zhang et al., 2006a). This approach revealed that many of the differentially expressed genes are critical for brain morphogenesis. These included genes involved in signaling pathways of Wnt, bone morphogenetic protein (Bmp) and retinoic acid (RA) (Zhang et al., 2006a). Wnt signaling pathways play an important role in early and late stages of brain development (Zhang et al., 2007). One of genes regulated by RFX4_v3 was Wnt-3a. Wnt-3a deficient mice show defects in growth and patterning of the dorsomedial cerebral cortex indicating that Wnt signaling regulates the development of the cerebral cortex (Lee et al., 2000; Louvi A and Wassef M, 2000; Huh et al., 2009). The Bmp signaling was described to be important for the patterning of the dorsal telencephalic midline and for the regulation of dorsal forebrain development (Furuta et al., 1997; Hébert et al., 2002; Liu and Niswander, 2005). RFX4_v3 down-regulates msh homeobox 3 (Msx3), a gene that belongs to a family of homeodomain transcriptional repressors (Zhang et al., 2006a). Together with Msx1 Msx3 has been shown to mediate Bmp signaling during neural tube development (Liu et al., 2004). The RA signaling pathway is also implicated in brain 
morphogenesis as shown by LaMantia and colleagues, who demonstrated that the pathway plays a role in forebrain development (LaMantia et al., 1993).

In addition to these pathways critical for brain morphogenesis, Zhang and colleagues identified transcription factors that have been shown before to be important for brain development, such as forkhead box A2 (Foxa2) and retina and anterior neural fold homeobox (Rax) (Zhang et al., 2006a; Nelander et al., 2009; Miranda-Angulo et al., 2014). In the same study CX3CL1 was found as direct target for RFX4_v3. The chemokine was significantly down-regulated in heads of RFX4_v3 -/- mice. The human and mouse promoters of CX3CL1 contain the highly conserved X-boxes, which are essential for RFX binding to DNA (Zhang et al., 2006a). Zhang and colleagues showed that RFX4_V3 directly binds to X-box 1 of the CX3CL1 promoter and activates the expression of this gene (Zhang et al., 2006a).

A yeast two-hybrid screening was performed to identify potential interaction partners of RFX4_v3 (Zhang et al., 2008). With this approach nine potential interaction partners were found, including the G-protein pathway suppressor 2 (GPS2), a nuclear factor (Zhang et al., 2008). Immunohistochemistry showed that GPS2 and RFX4_v3 co-localize in the nucleus and co-immunoprecipitation assays indicated a physical interaction between GPS2 and RFX4_v3. In addition, GPS2 was recruited by RFX4_v3 to the promoter of CX3CL1 indicating that GPS2 functions as transcriptional co-activator of RFX4_v3 (Zhang et al., 2008).

\subsection{Hydrocephalus}

Hydrocephalus is characterized by excessive accumulation of cerebrospinal fluid (CSF) inside the skull. The accumulation of CSF leads to abnormal dilation of the ventricles, which creates a harmful pressure level on the brain tissue. CSF is produced and secreted by the epithelial cells of the choroid plexus, which is located in the ventricles and subarachnoid space. The CSF production follows a cycle of constant production and reabsorption. The fluid circulates through the ventricular system, which is composed of two lateral ventricles, the third and fourth ventricle and the cerebral aqueduct. The CSF flows through the ventricles from the lateral to the third and via the cerebral aqueduct into the fourth ventricle. From the fourth ventricle the CSF flows via the median aperture of Magendie and the lateral aperture of Luschka into the subarachnoid space before being drained into the venous circulation (Ortloff et al., 2013). The balance between production and absorption of CSF is important. Conditions that block normal CSF flow or absorption can result in overproduction of CSF. All types of hydrocephalus are either communicating or non-communicating. In the communicating hydrocephalus the block of the CSF flow is mainly in the subarachnoid space distal to the ventricles, 
whereas in non-communicating hydrocephalus the impairment of the CSF flow is within the ventricles (Pérez-Fígares et al., 2001).

\subsubsection{Congenital hydrocephalus}

Congenital and acquired forms of hydrocephalus can be distinguished. Acquired hydrocephalus usually occurs postnatally. Possible causes are tumors, intraventricular or subarachnoid hemorrhage, and diseases such as meningitis and traumatic head injury (Pérez-Fígares et al., 2001; Sztriha et al., 2002; Gangemi et al., 2014).

Congenital hydrocephalus appears during fetal development and is already present at birth. The incidence of congenital hydrocephalus in humans is about 1-3 in every 1,000 live births (Pérez-Fígares et al., 1998; Huh et al., 2009).

Although the cause of congenital hydrocephalus is not well understood, it is believed that genetic abnormalities causing aqueductal stenosis and developmental disorders, which include neural tube defects, can lead to congenital hydrocephalus. Only one gene the X-linked L1 cell adhesion molecule (L1CAM) gene has been identified in humans to cause congenital hydrocephalus. Mutations in this gene are responsible for about $5 \%$ of the congenital cases with genetic cause (Bruni et al., 1988a; Sztriha et al., 2002; Zhang et al., 2006b). Apart from humans, congenital hydrocephalus occurs in several laboratory mammals. The analysis of several genetically modified animal models has led to the identification of more than 15 genes causing hydrocephalus in rodents (Rolf et al., 2001; Blackshear et al., 2003; Baas et al., 2006; Dietrich et al., 2009; Huh et al., 2009; Benadiba et al., 2012). Several studies support that stenosis of the aqueduct is a key event and the main cause for development of congenital hydrocephalus (Bruni et al., 1988a; Boillat et al., 1999; Jones and Klinge, 2008; Huh et al., 2009). The reason of stenosis is not well understood, but it was shown that dysfunction of the subcommissural organ during the fetal period may lead to aqueductal stenosis (Rodriguez et al., 1987; Rodríguez et al., 1998; Huh et al., 2009).

\subsubsection{Subcommissural organ and Reissner fiber glycoproteins}

The SCO is the a small brain gland located on the roof of the third ventricle, at the entrance of the cerebral aqueduct (Pérez-Fígares et al., 2001; Ortloff et al., 2013). This organ consists of secretory ependymal and hypependymal cells, which are arranged into 2 cell layers. It differentiates early in the ontogenesis and is active during throughout life (Naumann, 1986; Schoebitz et al., 1986; Pérez-Fígares et al., 1998). However, in humans the SCO differentiates very early during embryonic development. It is completely developed during the second half of the embryonic stage and is regressing during early childhood (Rodríguez et al., 1992). The SCO releases glycoproteins of high molecular 
weight into the ventricular CSF. In the CSF these glycoproteins condense to form a fibrous structure, which is known as Reissner fiber (RF). The RF grows in a rostracaudal direction by continuous addition of newly released glycoproteins. The condensed fibrous structure runs along the aqueduct, fourth ventricle, spinal cord and ends in the filum terminale. In the filum the RF glycoproteins undergo chemical changes before entering the bloodstream (Rodriguez et al., 1987; Nualart et al., 1991; Grondona et al., 1994).

In 1954, work by Overholser indicated that the secretion of SCO glycoproteins in the CSF during early embryonic stage prevents closure and stenosis of the aqueduct (Overholser et al., 1954). Therefore, it was suggested that maldevelopment of the SCO and resulting dysfunctional glycoprotein secretion lead to aqueductal stenosis and congenital hydrocephalus (Overholser et al., 1954; Newberne, 1962). Several publications support this hypothesis (Huh et al., 2009; Ortloff et al., 2013). It was shown that X-irradiation induced maldevelopment of the SCO during fetal life results in aqueductal stenosis and congenital hydrocephalus (Takeuchi and Takeuchi, 1986). In addition, immunization of pregnant rats with RF glycoproteins induced SCO defects, stenosis and hydrocephalus (Sara Rodriguez et al, 1999). Furthermore, several genetically modified animal models showed that maldevelopment of the SCO leads to the development of a congenital hydrocephalus (Huh et al., 2009). One example is the MT/Hokldr mouse, which lacks an SCO resulting in congenital hydrocephalus (Takeuchi et al., 1987). Likewise, the SUMS/np mice developed a hydrocephalus due to aqueductal stenosis and a small SCO (Jones et al., 1987; Bruni et al., 1988b). Lastly, severe morphological alterations of the SCO were observed in hydrocephalic human fetuses (Castañeyra-Perdomo et al., 1994).

The SCO originates from neuroepithelial cells that line the lumen of the dorsocaudal aspect of the diencephalon. These epithelial precursors of the SCO differentiate into specialized secretory ependymocytes in response to the induction of genes involved in the Wnt and bone morphogenetic protein (Bmp) signaling pathways (Liem et al., 1995; Liem et al., 1997). It was shown that SCO hypoplasia and dysfunction are associated with a number of genetic mutations including Wnt and Bmp signaling (Louvi $A$ and Wassef M, 2000; Bach, 2003; Ramos et al., 2004). One example is the transcription factor Msx1, which is induced by Bmp proteins and was shown to play an important role for dorsal neural patterning (Bach, 2003; Hayashi et al., 2006). The factor is expressed along the dorsal midline and neural tube and was also found in the SCO (Ramos et al., 2004). Deletion of both alleles leads to absence or malformation of the SCO, stenosis of the cerebral aqueduct, and development of a hydrocephalus (Fernández-Llebrez et al., 2004). 


\section{$1.4 \quad$ Aims}

\section{The role of astrocytic RFX4_v3 in demyelination}

The present work was inspired by a study of Cahoy and colleagues from 2008, who found the transcription factor RFX4 highly enriched in astrocytes (Cahoy et al., 2008). Our group previously identified RFX4_v3 as a highly up-regulated factor in MS lesions. The astrocytic expression of RFX4 was further confirmed by doubleimmunohistochemistry for RFX4 and GFAP.

Astrocytes are the most abundant cell type in MS lesions. In the last years it became increasingly clear that astrocytes do not only support neuronal functions, but - because of their immunological properties - also play an active role in the pathogenesis of MS lesions. On the one side, astrocytes support neurodegeneration and demyelination by promoting inflammation, but on the other side they can also create an environment conducive to regeneration and remyelination by secretion of pro-remyelinating factors (Williams et al., 2007; Nair et al., 2008; Brosnan and Raine, 2013).

Previous studies showed that members of the RFX family regulate genes important for the immune response (Gajiwala et al., 2000; Morotomi-Yano et al., 2002). RFX4_v3 directly targets and regulates the expression of CX3CL1 - a chemokine highly expressed in the brain and implicated in regulation of leukocyte migration and recruitment to MS lesions (Sunnemark et al., 2005; Zhang et al., 2006a; Broux et al., 2012; Blauth et al., 2015).

This raised the question whether an up-regulation of RFX4 in astrocytes acts as regulator of neuroinflammation, thereby influencing de- and remyelination in MS.

Therefore, the main aim of this thesis was to investigate the astrocytic role of RFX4 in de- and remyelination. For that purpose I used GFAP cell specific RFX4 conditional knockout mice to study the effect of RFX4_v3-deficiency on myelin loss and myelin repair in the cuprizone mouse model.

\section{Characterization of the GFAP cell specific RFX4 conditional knockout mice}

To generate GFAP cell specific RFX4 conditional knockout mice the RFX4 flox/flox strain was crossed with a GFAP-driven Cre transgenic strain. During the generation of the conditional knockout strain a substantial fraction of these animals developed a hydrocephalus.

Therefore, the second aim of this thesis was the phenotypic characterization of the newly generated GFAP cell specific RFX4 conditional knockout mice. To explain the 
pathological and functional basis of the hydrocephalus in RFX4 conditional knockout mice I performed histological and immunohistochemical analyses with a focus on the subcommissural organ, an ependymal brain gland that is important for the patency of the Sylvian aqueduct. To further understand the molecular mechanisms of the hydrocephalus formation I studied the expression of developmental genes which regulate dorsal midline structuring and the development of the SCO.

In the following, the first part of the thesis describes the characterization of the GFAP cell specific RFX4 conditional knockout mice, and the second part concerns the astrocytic role of RFX4_v3 in MS and the cuprizone mouse model. 


\section{Materials \& methods}

\section{$2.1 \quad$ Materials}

\subsubsection{Reagents}

Table 1: Reagents

Reagents

\section{Source of supply}

Agarose

Crystal violet

dNTP (desoxynucleoside triphosphate) mix

EDTA (ethylenediamine tetraacetic acid

disodiumsalt dihydrate)

GeneRuler $^{\mathrm{TM}}$, 1 kb Plus DNA ladder

HBSS (Hank's buffered salt solution) with $\mathrm{Ca}^{2+}$ and $\mathrm{Mg}^{2+}$

Isopropyl alcohol

PBS (phosphate buffered salt solution), sterile

Penicillin, 10,000 units / streptomycin, 10mg/ml

Trypan blue

BSA (bovine serum albumine)

Cuprizone

(Bis(cyclohexanone)oxaldihydrazone)

DAB (3,3'-diaminobenzidine)

DAPI (4',6-diamidino-2-phenylindole)

Percoll

DePex mounting medium

Dako Fluorescent Mounting Medium

Ethanol, 96\%

PLL (poly-L-lysine hydro bromide), powder

Formalin (37\% solution, free from acid)

Horse serum

LFB (Luxol Fast Blue)

Mayer's hemalum solution

Methylene blue

$\mathrm{NaOH}$

$\mathrm{NaOH}(1 \mathrm{~mol} / \mathrm{I}, 0,1 \mathrm{~mol} / \mathrm{l})$

$\mathrm{NaCl}$

PBS (Dulbecco's PBS, 10x powder)

Periodic acid

ExtrAvidin-Peroxidase

PFA (paraformaldehyde)
StarLab GmbH, Hamburg, Germany

Sigma Aldrich, St. Louis, MO, USA

Thermo Scientific

Carl Roth, Karlsruhe, Germany

Thermo Scientific, Waltham, MA, USA

PAA,Pasching, Germany

Merck Millipore, Darmstadt, Germany

Gibco, Germany

Gibco, Germany

Sigma Aldrich, St. Louis, MO, USA

Serva Electrophoresis $\mathrm{GmbH}$, Germany

Sigma-Aldrich, Germany

Sigma Aldrich, Germany

Molecular Probes, life technologies,

Germany

Sigma Aldrich, Germany

Serva Electrophoresis GmbH, Germany

Dako Deutschland GmbH, Germany

Merck, Millipore, Germany

Sigma Aldrich, Germany

Merck, Millipore, Germany

PAA,Pasching, Germany

Sigma-Aldrich, Germany

Merck, Millipore, Germany

Merck, Millipore, Germany

Merck, Millipore, Germany

Merck KGaA, Germany

Merck KgaA, Germany

Applichem, Germany

Merck KgaA, Germany

Sigma Aldrich, Germany

Sigma Aldrich, Germany 
Reagents

Qiazol Lysis Reagent

Random Hexamers

Triton $\times 100$

Trizma base

PCR buffer, 5x Green GoTaq Reaction Buffer

FCS (fetal calf serum)

$\mathrm{H}_{2} \mathrm{O}_{2}$ (30\% solution)

BME Medium (Eagle's Basal Medium)

GlutaMax

Ground mouse chow (complete feed for rat

$\&$ mice maintenance, ground)

$\mathrm{HCl}(25 \%, 1 \mathrm{~mol} / \mathrm{l}, 0.1 \mathrm{~mol} / \mathrm{l})$

DMEN-Dulbecco's Modified Eagle Medium

HEPES (4-(2-hydroxyethyl)-1-

Naphthol AS phosphate

Levamisole

Fast blue BB salt
Source of supply

Qiagen, Germany

Invitrogen, life technologies, Germany

MP Biomedicals, Germany

Sigma Aldrich, Germany

Promega, Germany

Sigma Aldrich, St. Louis, MO, USA

Merck, Millipore, Germany

Gibco, Germany

Gibco, Germany

Ssniff Spezialdiäten GmbH, Germany

Merck, Millipore, Germany

Gibco, Germany

Sigma Aldrich, St. Louis, MO, USA

Sigma Aldrich, St. Louis, MO, USA

MP Biomedicals, LLC, Germany

Sigma Aldrich, St. Louis, MO, USA

\section{Abbreviations:}

PCR: Polymerase chain reaction

\subsubsection{Solutions, buffers and cell culture media}

Table 2: Solutions, buffers and cell culture media Solution

FACS (fluorescence-activated cell sorting) buffer

MACS (magnetic-activated cell sorting) buffer

DMEM $_{\text {FACS }}$ buffer

Tris-EDTA buffer

\section{Composition}

PBS, sterile

$2 \%$ FCS

PBS, sterile

0.05\% BSA

2 mM EDTA

pH 7.2

DMEM

$2 \%$ FCS

$10 \mathrm{mM}$ Tris

1 mM EDTA

pH 9.0 
Solution

LFB working solution

Tail lysis buffer

TAE buffer

PFA, 4\% solution

$\mathrm{HCl}$-Isopropanol

Citric acid buffer (pH 6.0)

DAB working solution

Levamisole (stock solution)

Tris HCL buffer
Composition

$0.1 \%$ LFB (w/ v)

$0.5 \%$ acetic acid in Ethanol

Bidistilled water

5 mM EDTA

$200 \mathrm{mM} \mathrm{NaCl}$

$0.1 \mathrm{M}$ Tris- $\mathrm{HCl} \mathrm{pH} 8.5$

$0.2 \%$ SDS

pH 8.5

Bidistilled water

$40 \mathrm{mM}$ Tris acetate

$1 \mathrm{mM}$ EDTA

$\mathrm{pH} 8.0$

40 g PFA

$1000 \mathrm{ml}$ 1-fold PBS

$\mathrm{NaOH}$

$\mathrm{pH} 7.4$

70\% Isopropyl alcohol

$0.25 \% \mathrm{HCl}$

10 mM Citric acid

$\mathrm{NaOH}$

pH6.0

$49 \mathrm{ml}$ PBS

$1 \mathrm{ml} \mathrm{DAB}$

$20 \mu$ l hydrogen peroxide

$1 \mathrm{M}$ Levamisole

bidistilled water

$1 \mathrm{M}$ Tris HCL

$1 \mathrm{M} \mathrm{HCL}$

pH 8.2 


\section{Solution}

Naphthol AS phosphate mix (stock solution)

Fast blue

Chloral hydrate, $14 \%$ solution

$\mathrm{BME}_{\text {complete }}$

Dispase II (stock solution)

Collagenase D (stock solution)

DNase I (stock solution)

\section{Composition}

$10 \mathrm{mg} / \mathrm{ml}$ Naphthol AS phosphate mix

$\mathrm{N}, \mathrm{N}$-di-methylformamide

$0.5 \mathrm{mg} / \mathrm{ml}$ Fast Blue BB salt

$0.2 \mathrm{mg} / \mathrm{ml}$ Naphthol AS phosphate

$1 \mathrm{mM}$ Levamisole

$0.1 \mathrm{M}$ Tris HCL buffer, $\mathrm{pH} 8.2$

$14 \mathrm{~g}$ Chloral hydrate

$100 \mathrm{ml}$ bidistilled water

BME

$10 \%$ Horse serum

100 units Penicillin

$0.1 \mathrm{mg} / \mathrm{ml}$ Streptomycin

2 mM GlutaMax

$10 \mathrm{mg} / \mathrm{ml}$ Dispase II

50 mM HEPES

100 mg/ml Collagenase D

Steril HBSS

$5 \mathrm{mg} / \mathrm{ml}$ DNase I

Steril HBSS

\begin{abstract}
Abbreviation:
BME: Eagle's Basal Medium; DAB: 3,3'-diaminobenzidine BSA: bovine serum albumin; EDTA: ethylenediamine tetraacetic acid disodiumsalt dehydrate; PBS: phosphate buffered salt solution; Tris: tris(hydroxymethyl)aminomethane; LFB: Luxol Fast Blue; PFA: paraformaldehyde; DNase: desoxyribonuclease; TAE: Tris-acetate-EDTA
\end{abstract}

\title{
2.1.3 Enzymes/ proteins
}

Table 3: Enzymes/ proteins

\section{Enzymes/ proteins}

ExtrAvidin-Peroxidase (0.01\% solution)

Proteinase $\mathrm{K}$

Trypsin-EDTA (0.05\%)

BSA (bovine serum albumin)

Collagenase D

\section{Source of supply}

Sigma Aldrich, Germany

Sigma Aldrich, St. Louis, MO, USA

Gibco, life technologies, Germany

SERVA Electrophoresis GmbH, Heidelberg,

Germany

Roche, Basel, Switzerland 
Enzymes/ proteins

Dispase

DNase I

$\mathrm{N}$-glycosylase

\subsubsection{Kits}

Table 4: Kits

Kits

qPCR Core Kit

RNeasy Mini Kit

TaqMan MicroRNA Reverse Transcription

Kit

TopTaq Master Mix Kit
Source of supply

Roche, Basel, Switzerland

Worthington, Lakewood, USA

Eurogentic, Germany

Abbreviations:

RNA: ribonucleic acid

\subsubsection{Monoclonal antibodies for flow cytometry}

Table 5: Monoclonal antibodies for flow cytometry

\begin{tabular}{lllll} 
Specificity & Fluorochrome & Clone & Dilution & Source of supply \\
\hline CD86 & APC Cy7 & GL1 & $1: 200$ & Biolegend \\
H-2Kb (MHCI) & FITC & AF6-88.5 & $1: 200$ & BD Pharmagen \\
CD45 & PerCP & $30-F 11$ & $1: 200$ & BD Pharmagen \\
CD11b & BV421 & M1/70 & $1: 200$ & Biolegend \\
CD80 & PE & $16-10 A 1$ & $1: 200$ & Biolegend \\
CD16/32 & - & 93 & $1: 100$ & Biolegend \\
\hline
\end{tabular}

\section{Abbreviations:}

CD: cluster of differentiation; $\mathbf{M H C l}$ : major histocompatibility complex class I molecule; FITC: fluorescein isothiocyanate; PE: phycoerythrin; PerCP: peridinin chlorophyll protein APC: allophycocyanin; BV: brilliant violet; APC Cy7: allophycocyanin cyanine 7

\subsubsection{Primary antibodies for immunohistochemistry and fluorescence immunohistochemistry}

Table 6: Primary antibodies for immunohistochemistry and fluorescence immunohistochemistry

\begin{tabular}{lllllll} 
Antibody & Marker for & $\begin{array}{l}\text { Species/ } \\
\text { Clonalityl } \\
\text { antigen }\end{array}$ & Dilution & $\begin{array}{l}\text { Antigen } \\
\text { retrieval }\end{array}$ & $\begin{array}{l}\text { Blocking } \\
\text { buffer }\end{array}$ & $\begin{array}{l}\text { Source of } \\
\text { supply }\end{array}$ \\
\hline GFAP & Activated & Rabbit/ & $1: 1000$ & None & $10 \%$ FCS/ & DAKO \\
& astrocytes & polyclonal/ Z0334 & & & PBS &
\end{tabular}




\begin{tabular}{|c|c|c|c|c|c|c|}
\hline Antibody & Marker for & $\begin{array}{l}\text { Species/ } \\
\text { Clonalityl } \\
\text { antigen }\end{array}$ & Dilution & $\begin{array}{l}\text { Antigen } \\
\text { retrieval }\end{array}$ & $\begin{array}{l}\text { Blocking } \\
\text { buffer }\end{array}$ & $\begin{array}{l}\text { Source of } \\
\text { supply }\end{array}$ \\
\hline GFAP & $\begin{array}{l}\text { Activated } \\
\text { astrocytes }\end{array}$ & $\begin{array}{l}\text { Mouse/ Mono- } \\
\text { clonal/ 134B1 }\end{array}$ & $1: 300$ & $\begin{array}{l}\text { Microwave } \\
\text { citric acid } \\
\text { buffer }\end{array}$ & $\begin{array}{l}10 \% \mathrm{FCS} / \\
\text { PBS }\end{array}$ & $\begin{array}{l}\text { Synaptic } \\
\text { Systems }\end{array}$ \\
\hline CNPase & $\begin{array}{l}\text { Myelin and } \\
\text { oligodendrocyte } \\
\text { protein }\end{array}$ & $\begin{array}{l}\text { Mouse/ Mono- } \\
\text { clonal/ } \\
\text { SMI-91R }\end{array}$ & $1: 200$ & $\begin{array}{l}\text { Microwave } \\
\text { citric acid } \\
\text { buffer }\end{array}$ & $\begin{array}{l}10 \% \mathrm{FCS} / \\
\text { PBS }\end{array}$ & Covance \\
\hline Olig2 & $\begin{array}{l}\text { OPCs and mature } \\
\text { oligodendrocyte }\end{array}$ & Rbb/ Poly-clonal & $1: 300$ & $\begin{array}{l}\text { Microwave } \\
\text { Tris-EDTA }\end{array}$ & $\begin{array}{l}10 \% \mathrm{FCS} / \\
\text { PBS }\end{array}$ & IBL \\
\hline p25/TPPP & $\begin{array}{l}\text { mature } \\
\text { oligodendrocyte }\end{array}$ & $\begin{array}{l}\text { Rbb/ Poly-clonal/ } \\
\text { EPR3316 }\end{array}$ & $1: 100$ & $\begin{array}{l}\text { Microwave } \\
\text { Tris-EDTA }\end{array}$ & $\begin{array}{l}10 \% \mathrm{FCS} / \\
\text { PBS }\end{array}$ & Abcam \\
\hline Ki-67 & $\begin{array}{l}\text { Marker of } \\
\text { proliferating cells }\end{array}$ & $\begin{array}{l}\text { Rat/ Mono-clonal/ } \\
\text { Tec3 }\end{array}$ & $1: 20$ & $\begin{array}{l}\text { Microwave } \\
\text { citric acid } \\
\text { buffer }\end{array}$ & $\begin{array}{l}10 \% \mathrm{FCS} / \\
\text { PBS }\end{array}$ & DAKO \\
\hline Mac3 & activated microglia & $\begin{array}{l}\text { Rat/ Poly- } \\
\text { clonal/M3/84 }\end{array}$ & $1: 200$ & $\begin{array}{l}\text { Microwave } \\
\text { citric acid } \\
\text { buffer }\end{array}$ & $\begin{array}{l}10 \% \mathrm{FCS} / \\
\text { PBS }\end{array}$ & $\begin{array}{l}\text { BD } \\
\text { Pharmingen }\end{array}$ \\
\hline RFX4 & $\begin{array}{l}\text { Regulatory factor } X \\
4\end{array}$ & $\begin{array}{l}\text { Rbb/ Poly-clonal/ } \\
\text { aa352-401 (LS- } \\
\text { C31698) }\end{array}$ & $1: 100$ & $\begin{array}{l}\text { Microwave } \\
\text { citric acid } \\
\text { buffer }\end{array}$ & $\begin{array}{l}10 \% \mathrm{FCS} / \\
\text { PBS }\end{array}$ & $\begin{array}{l}\text { LifeSpan } \\
\text { BioSciences }\end{array}$ \\
\hline APP & $\begin{array}{l}\text { Amyloid precursor } \\
\text { protein, early } \\
\text { axonal damage }\end{array}$ & $\begin{array}{l}\text { Mouse/ Mono- } \\
\text { clonal/ MAB348 }\end{array}$ & $1: 2,000$ & $\begin{array}{l}\text { Microwave } \\
\text { citric acid } \\
\text { buffer }\end{array}$ & $\begin{array}{l}10 \% \mathrm{FCS} / \\
\text { PBS }\end{array}$ & $\begin{array}{l}\text { Merck } \\
\text { Millipore }\end{array}$ \\
\hline AFRU & $\begin{array}{l}\text { Reissner fiber } \\
\text { glycoproteins }\end{array}$ & $\begin{array}{l}\text { Rabbit/ Poly- } \\
\text { clonal/ FRU }\end{array}$ & $1: 1,000$ & None & $\begin{array}{l}10 \% \mathrm{FCS} / \\
\text { PBS }\end{array}$ & $\begin{array}{l}\text { Gift from } \\
\text { Esteban } \\
\text { Rodriguez }\end{array}$ \\
\hline Caspase3 & $\begin{array}{l}\text { Activated } \\
\text { caspase3, } \\
\text { apoptosis }\end{array}$ & $\begin{array}{l}\text { Rabbit/ Poly- } \\
\text { clonal/ } \\
\text { C92-605 }\end{array}$ & $1: 150$ & $\begin{array}{l}\text { Microwave } \\
\text { Tris-EDTA }\end{array}$ & $\begin{array}{l}10 \% \mathrm{FCS} / \\
\text { PBS }\end{array}$ & $\begin{array}{l}\text { BD } \\
\text { Biosciences }\end{array}$ \\
\hline
\end{tabular}




\begin{tabular}{lllllll} 
Antibody & Marker for & $\begin{array}{l}\text { Species/ } \\
\text { Clonalityl } \\
\text { antigen }\end{array}$ & Dilution & $\begin{array}{l}\text { Antigen } \\
\text { retrieval }\end{array}$ & $\begin{array}{l}\text { Blocking } \\
\text { buffer }\end{array}$ & $\begin{array}{l}\text { Source of } \\
\text { supply }\end{array}$ \\
\hline MBP & myelin protein & $\begin{array}{l}\text { Rabbit/ Poly- } \\
\text { clonal/ } 62301\end{array}$ & $1: 1,000$ & None & $\begin{array}{l}10 \% \text { FCS/ } \\
\text { DAKO }\end{array}$
\end{tabular}

\begin{abstract}
Abbreviations:
GFAP: glial fibrillary acidic protein; CNPase: 2',3'-Cyclic-nucleotide 3'-phosphodiesterase; Olig: oligodendrocyte transcription factor; p25/TPPP: brain-specific 25-kDa protein; Ki: Kiel; Mac: macrophage; APP: amyloid precursor protein; FRU: fiber reissner urea; AFRU: antiserum against fiber reissner urea; MBP: myelin basic protein

\subsubsection{Secondary antibodies for immunohistochemistry and fluorescence immunohistochemistry}

Table 7: Secondary antibodies for immunohistochemistry and fluorescence immunohistochemistry

\begin{tabular}{|c|c|c|c|c|}
\hline Antibody & Conjugation & Host & Dilution & Source of supply \\
\hline \multirow[t]{2}{*}{ Anti rabbit IgG } & Cy3 conjugated & goat & $1: 100$ & Jackson \\
\hline & & & & ImmunoResearch \\
\hline \multirow[t]{2}{*}{ Anti mouse $\lg G$} & Cy2 conjugated & goat & $1: 100$ & Jackson \\
\hline & & & & ImmunoResearch \\
\hline \multirow[t]{2}{*}{ Anti-rabbit IgG } & biotinylated & goat & $1: 200$ & Jackson \\
\hline & & & & ImmunoResearch \\
\hline Anti-mouse IgG & biotinylated & sheep & $1: 200$ & GE Healthcare \\
\hline Anti-rat IgG & biotinylated & goat & $1: 500$ & $\begin{array}{l}\text { DCS Innovative } \\
\text { diagnostic system }\end{array}$ \\
\hline Anti-mouse IgG & $\begin{array}{l}\text { Alkaline } \\
\text { phosphatase }\end{array}$ & Goat & $1: 50$ & DAKO \\
\hline
\end{tabular}

\title{
Abbreviations:
}

IgG: immunoglobulin G; Cy3: cyanine 3; Cy2: cyanine 2

\subsubsection{Oligonucleotide primers}

Table 8: Oligonucleotide primers

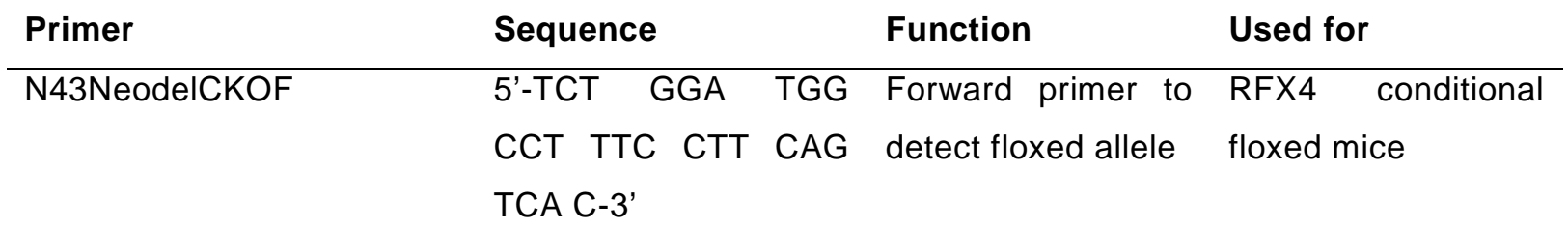

N43NeodelCKOR

5'-AAC GGT GTG Reverse primer to RFX4 conditional AGG GTT GTG GCA detect floxed allele floxed mice 


\begin{tabular}{|c|c|c|c|}
\hline Primer & Sequence & Function & Used for \\
\hline $\begin{array}{l}\text { GFAPCRE TG } \\
\text { olMR1084 }\end{array}$ & $\begin{array}{l}\text { 5‘-GCG GTC TGG } \\
\text { CAG TAA AAA CTA } \\
\text { TC-3‘ }\end{array}$ & $\begin{array}{l}\text { Forward primer to } \\
\text { detect cre }\end{array}$ & $\begin{array}{l}\text { GFAP cre mice } \\
73.12 \mathrm{Mvs} / \mathrm{J}\end{array}$ \\
\hline $\begin{array}{l}\text { GFAPCRE TG } \\
\text { olMR1085 }\end{array}$ & $\begin{array}{l}\text { 5'-GTG AAA CAG } \\
\text { CAT TGC TGT CAC } \\
\text { TT-3' }\end{array}$ & $\begin{array}{l}\text { Reverse primer to } \\
\text { detect cre }\end{array}$ & $\begin{array}{l}\text { GFAP cre mice } \\
73.12 \mathrm{Mvs} / \mathrm{J}\end{array}$ \\
\hline RFX4 variant v3 Fw & $\begin{array}{l}\text { 5'-ATG CAT TGT } \\
\text { GGG TTA CTG GAG-3' }\end{array}$ & $\begin{array}{l}\text { Forward primer to } \\
\text { detect RFX4_v3 }\end{array}$ & RFX4 variant v3 \\
\hline RFX4 variant v3 Rev & $\begin{array}{l}\text { 5‘-TGA ATA TGC CAC } \\
\text { TGT CTG TTT G-3’ }\end{array}$ & $\begin{array}{l}\text { Reverse primer to } \\
\text { detect RFX4_v3 }\end{array}$ & RFX4 variant v3 \\
\hline RFX4 variant v2/v4 FW & $\begin{array}{l}\text { 5'-GGG CTT CTC CAA } \\
\text { АCT CCT GT-3' }\end{array}$ & $\begin{array}{l}\text { Forward primer to } \\
\text { detect RFX4 v2/v4 }\end{array}$ & RFX4 variant v2/v4 \\
\hline RFX4 variant v2/v4 Rev & $\begin{array}{l}\text { 5'-AGC CAC TTT TAG } \\
\text { CCA СТC АTC-3' }\end{array}$ & $\begin{array}{l}\text { Reverse primer to } \\
\text { detect RFX4 v2/v4 }\end{array}$ & RFX4 variant v2/v4 \\
\hline RFX4 variant v1 FW & $\begin{array}{l}\text { 5'-GCA GAA ATA TCA } \\
\text { CGG AAT GGT-3’ }\end{array}$ & $\begin{array}{l}\text { Forward primer to } \\
\text { detect RFX4 v1 }\end{array}$ & RFX4 variant v1 \\
\hline RFX4 variant v1 Rev & $\begin{array}{l}\text { 5‘-AGC CAC TTT TAG } \\
\text { CCA CTC ATC-3’ }\end{array}$ & $\begin{array}{l}\text { Reverse primer to } \\
\text { detect RFX4 v1 }\end{array}$ & RFX4 variant v1 \\
\hline
\end{tabular}

\section{Source of supply:}

Oligonucleotide primers were ordered from Metabion, Planegg/Steinkirchen, Germany.

\subsubsection{TaqMan Expression Assays}

Table 9: TaqMan Expression Assays

\begin{tabular}{lll} 
Gene symbol & Gene name & Source of supply \\
\hline GapDH & Glyceraldehyde-3-phosphate & Applied Biosystems, life technologies/ \\
& dehydrogenase & Mm99999915_g1 \\
RFX4 & Regulatory factor X 4 & Applied Biosystems, life technologies/ \\
& & Mm01240658_m1 \\
GFAP & Glial fibrillary acidic protein & Applied Biosystems, life technologies/ \\
& & Mm01253033_m1
\end{tabular}




\begin{tabular}{|c|c|c|}
\hline Gene symbol & Gene name & Source of supply \\
\hline TNF- $\alpha$ & Tumor necrosis factor alpha & $\begin{array}{l}\text { Applied Biosystems, life } \\
\text { technologies/Mm00443258_m1 }\end{array}$ \\
\hline CXCL10 & $\begin{array}{l}\text { Chemokine (C-X-C motif) ligand } \\
10\end{array}$ & $\begin{array}{l}\text { Applied Biosystems, life } \\
\text { technologies/Mm00445235_m1 }\end{array}$ \\
\hline CCL2 & Chemokine ( $\mathrm{C}-\mathrm{C}$ motif) ligand 2 & $\begin{array}{l}\text { Applied Biosystems, life } \\
\text { technologies/ Mm00441242_ml }\end{array}$ \\
\hline CCL3 & Chemokine ( $\mathrm{C}-\mathrm{C}$ motif) ligand 3 & $\begin{array}{l}\text { Applied Biosystems, life } \\
\text { technologies/ Mm00441259_gl }\end{array}$ \\
\hline CXCL12 & $\begin{array}{l}\text { Chemokine (C-X-C motif) ligand } \\
12\end{array}$ & $\begin{array}{l}\text { Applied Biosystems, life } \\
\text { technologies/Mm00445553_m1 }\end{array}$ \\
\hline$I L-1 ß$ & Interleukin 1 beta & $\begin{array}{l}\text { Applied Biosystems, life } \\
\text { technologies/Mm00434228_m1 }\end{array}$ \\
\hline Wnt3 & $\begin{array}{l}\text { Wingless-type MMTV integration } \\
\text { site family, member } 3\end{array}$ & $\begin{array}{l}\text { Applied Biosystems, life } \\
\text { technologies/Mm00437336_m1 }\end{array}$ \\
\hline Wnt3a & $\begin{array}{l}\text { Wingless-type MMTV integration } \\
\text { site family, member } 3 a\end{array}$ & $\begin{array}{l}\text { Applied Biosystems, life } \\
\text { technologies/Mm00437337_m1 }\end{array}$ \\
\hline Foxa2 & Forkhead box A2 & $\begin{array}{l}\text { Applied Biosystems, life } \\
\text { technologies/Mm01976556_s1 }\end{array}$ \\
\hline Ctnnb1 & Beta-catenin-1 & $\begin{array}{l}\text { Applied Biosystems, life } \\
\text { technologies/Mm00495976_m1 }\end{array}$ \\
\hline Fzd10 & Frizzled class receptor 10 & $\begin{array}{l}\text { Applied Biosystems, life } \\
\text { technologies/Mm00558396_s1 }\end{array}$ \\
\hline Rspo1 & R-spondin 1 & $\begin{array}{l}\text { Applied Biosystems, life } \\
\text { technologies/Mm00507077_m1 }\end{array}$ \\
\hline Vtn & Vitronectin & $\begin{array}{l}\text { Applied Biosystems, life } \\
\text { technologies/Mm00483039_m1 }\end{array}$ \\
\hline CX3CL1 & Chemokine (CX3C motif) ligand 1 & $\begin{array}{l}\text { Applied Biosystems, life } \\
\text { technologies/Mm00436454_m1 }\end{array}$ \\
\hline
\end{tabular}

FAM labeled primers/probes were selected to be intron spanning.

\subsubsection{Consumables}

Table 10: Consumables

Consumable

96 PCR Plate Biosphere Plus

Tubes (50 ml, $15 \mathrm{ml}, 2 \mathrm{ml}, 1,5 \mathrm{ml}, 0.2 \mathrm{ml}$ )

Cell culture flask, $25 \mathrm{~cm}^{2}$

MS columns

Bottle top filter, $0.2 \mu \mathrm{m}$

\section{Source of supply}

Sarstedt

Sarstedt, Nuembrecht, Germany

Greiner bio-one, Kremsmuenster, Austria

Miltenyi Biotec, Bergisch Gladbach,

Germany

Sarstedt, Nuembrecht, Germany 


\section{Consumable}

Bottle top filter, $0.2 \mu \mathrm{m}$

Cryogenic tubes

FACS tube, $5 \mathrm{ml}$

Needles

Syringes

Syringes

Nunc $^{\mathrm{TM}}$ MicroWell ${ }^{\mathrm{TM}}$ Plates, 96 well round bottom

Bottle top filter, $0.2 \mu \mathrm{m}$

Glass microscope slides (Superfrost Plus)

24-well tissue culture plates, sterile

BD Falcon ${ }^{\mathrm{TM}}$ tube, $50 \mathrm{ml}$

BD Falcon ${ }^{\mathrm{TM}}$ tube, $15 \mathrm{ml}$

$70 \mu \mathrm{m}$ cell strainer

\section{Source of supply}

Sarstedt, Nuembrecht, Germany

Thermo Scientific, Waltham, MA, USA

BD Biosciences, Franklin Lakes, NJ, USA

BD Biosciences, Franklin Lakes, NJ, USA

BD Biosciences, Franklin Lakes, NJ, USA

B Braun, Melsungen, Germany

Thermo Scientific, Waltham, MA, USA

Sarstedt, Nuembrecht, Germany

Thermo Scientific, USA

Greiner bio-one, Kremsmuenster, Austria

BD Biosciences, Germany

BD Biosciences, Germany

BD Biosciences, Germany

\section{Abbreviations}

FACS: fluorescence-activated cell sorting

\subsubsection{Technical devices}

Table 11: Technical devices

Device

Cell incubator CWJ300DABA

Neubauer chamber

Centrifuge $5415 \mathrm{R}$

Centrifuge $5810 \mathrm{R}$

FACS Aria ${ }^{\mathrm{TM}} \|$

Ocular counting grid, WHSZ 10X-H

Microtome SM2000R

Microwave NN E201 WM

Light microscope BX41

iQ5 Real-Time PCR Detection System

Thermo mixer comfort

UV transluminator

T3 Thermocycler

Speed vacuum Concentrator 5301

Fluorescence microscope BX51

OctoMACS ${ }^{\mathrm{TM}}$ separator

Camera for fluorescence microscope XM10

Camera for light microscope DP71

\section{Source of supply}

Cellstar, Nunc GmbH, Germany

Superior Marienfeld, Lauda-Koenigshofen, Germany

Eppendorf, Hamburg, Germany

Eppendorf, Hamburg, Germany

BD Biosciences, Franklin Lakes, NJ, USA

Olympus, Germany

Leica, Germany

Panasonic, Germany \& Austria

Olympus, Germany

Bio-Rad, Germany

Eppendorf, Hamburg, Germany

Vilber Lourmat, Eberhardzell, Germany

Biometra, Germany

Eppendorf, Hamburg, Germany

Olympus, Germany

Miltenyi Biotec, Bergisch Gladbach,

Germany

Olympus, Germany

Olympus, Germany 
Device

Mastercycler gradient

Excelsior AS tissue processor

Microscop IX51
Source of supply

Eppendorf, Hamburg, Germany

Thermo scientific

Olympus, Germany

Abbreviations:

FACS: fluorescence-activated cell sorting; MACS: magnetic-activated cell sorting

\subsubsection{Software}

Table 12: Software

Software

GraphPad Prism 5.01

FlowJo 7.6.1

BD FACSDiva Software 6.1.2

PSRemote 1.6.5

AnalysisTM

CellSensDimension 1.7

iQ5 Optical System Software
Source of supply

GraphPad, USA

Tree Star Inc., Ashland, OR, USA

BD Biosciences, Franklin Lakes, NJ, USA

Breeze systems limited, Camberley, UK

Olympus, Germany

Olympus, Germany

Bio-Rad, Germany

\subsubsection{Tissue from MS patients}

Table 13: Tissue from MS patients

\begin{tabular}{llll} 
Case-number & Age & Sex & MS lesion \\
\hline B515/10K-2 & 31 & $\mathrm{~m}$ & Early active \\
A20/12 & 63 & $\mathrm{f}$ & Chronic inactive
\end{tabular}

The types of MS lesions were determined by Dr. Andreas Junker and Prof. Christine Stadelmann.

\section{$2.2 \quad$ Animals}

\subsection{Mouse strains}

All mutant mice were generated from in house breeding colonies at the animal facility of the University Medical Center Goettingen.

\section{C57BI/6J mice}

Seven- to eight-week-old $\mathrm{C} 57 \mathrm{BI} / 6 \mathrm{~J}$ mice were purchased from Charles River laboratories, Sulzfeld, Germany or from the animal facility of the University Medical Center Goettingen. C57BL/6J P0 mice were provided by the animal facility of the University Medical Center Goettingen. 


\section{RFX4 flox/flox}

The RFX4 flox/flox mice were generated and provided by Dr. Perry Blackshear. This line was backcrossed to C57BL/6 animals. This strain contains LoxP sites flanking the exon 4 of the RFX4 gene.

\section{GFAP-Cre 73.12 mice}

The GFAP cre line 73.12 was purchased from Charles River, Germany. GFAP cre 73.12 mice are generated and characterized by Michael V Sofroniew in 2004 (Garcia, A Denise R et al., 2004). This line was backcrossed to C57BL/6 animals for at least 14 generations. Cre recombinase activity under control of the GFAP promoter is targeted to ependymal cells and to most astrocytes in the healthy and injured brain and spinal cord.

\section{GFAP cell specific RFX4 conditional knockout mice}

To generate GFAP cell specific RFX4 conditional knockout mice, the GFAP cre line 73.12 was crossed with the RFX4 flox/flox mice, which results in RFX4 deletion under control of the GFAP promoter. The exon 4 of RFX4 is shared with variant 2, and variant 3 of RFX4. Variant 2 of RFX4 has been identified in the testis, while the RFX4 variant 3 has been only identified in the brain (Blackshear et al., 2003; Matsushita et al., 2005). Therefore and due to the hydrocephalus phenotype of the GFAP cell specific conditional RFX4 knockout mice the strain was designated as RFX4_v3 conditional knockout mice in the discussion of the phenotype of GFAP cell specific conditional RFX4 knockout mice and in the second part of my thesis.

\subsubsection{Housing}

Animals were housed in the animal facility of the University Medical Center Goettingen. Up to 7 mice were housed together on 12/12h light/ dark cycle and had access to food and water ad libitum under SPF conditions. Animals were adapted to the new environment for at least one week before the experiments were started. All animal experiments were performed in accordance with the European Communities Council Directive of 24 November 1986 (86/EEC) and were authorized by the Government of Lower Saxony, Germany. 


\section{$2.4 \quad$ Methods \\ 2.4.1 Genotyping}

\section{DNA extraction}

For genotyping, genomic DNA was extracted from tail biopsies of RFX4 flox/flox mice, GFAP cre 73.12 mice and GFAP cell specific RFX4 knockout mice. Therefore, the tail biopsies were lysed in $350 \mu$ tail lysis puffer with proteinase $K(1: 100)$ for at least 2.5 hours in a thermo mixer $\left(350 \mathrm{rpm}, 56^{\circ} \mathrm{C}\right)$. The digested tissue was centrifuged for $10 \mathrm{~min}$ at maximum speed to remove remaining tissue. The genomic DNA in the supernatant was precipitated with $350 \mu \mathrm{l}$ isopropyl alcohol and centrifuged for $10 \mathrm{~min}$ at maximum speed. The pellet was washed with $700 \mu \mathrm{l} 70 \%$ ethanol and centrifuged for $5 \mathrm{~min}$ at maximum speed. The supernatant was discarded and the pellet was dried in a speed vacuum concentrator. The dried pellet was resuspended in $100 \mu$ l bidistilled water.

\section{Genotyping of RFX4 flox/flox and GFAP specific RFX4 conditional knockout mice}

PCR reaction was performed with the TopTaq buffer and TopTaq DNA Polymerase.

Each PCR reaction included:

\section{Volume Component}

$10 \mu \mathrm{l} \quad$ genomic DNA

$0.5 \mu \mathrm{l} \quad 10 \mathrm{mM}$ dNTP mix

$2.5 \mu \mathrm{l} \quad 10 \times$ TopTaq buffer

$0.5 \mu \mathrm{l} \quad 10 \mathrm{pmol}$ oligonucleotide primer (N43NeodelCKOF/ N43NeodelCKOR)

$0.125 \mu \mathrm{l} \quad$ TopTaq DNA polymerase

$10,9 \mu \mathrm{l} \quad$ bidistilled water

PCR reactions were run in the Eppendorf thermocycler (Mastercycler gradient) at the following cycling conditions: 


\begin{tabular}{|c|c|c|}
\hline Temperature & Time & Step \\
\hline $94^{\circ} \mathrm{C}$ & $3 \mathrm{~min}$ & Initial denaturation \\
\hline $94^{\circ} \mathrm{C}$ & $30 \mathrm{~s}$ & Denaturation \\
\hline $60^{\circ} \mathrm{C}$ & 35 cycles & Annealing \\
\hline $72^{\circ} \mathrm{C}$ & $1 \mathrm{~min}$ & Extension \\
\hline $72^{\circ} \mathrm{C}$ & $10 \min$ & Final extension \\
\hline $4^{\circ} \mathrm{C}$ & $\infty$ & storage \\
\hline
\end{tabular}

To analyze the amplified PCR product, the samples $(10 \mu \mathrm{l})$ were loaded on an agarose gel in TAE buffer containing ethidium bromide. The endogenous allele was characterized by a band at $280 \mathrm{bp}$, while the floxed allele was detected by a band at $420 \mathrm{bp}$. Both bands were detected in heterozygous animals.

Genotyping of GFAP cre 73.12 mice and GFAP specific RFX4 conditional knockout mice

Each PCR reaction included:

\section{Volume Component}

$2 \mu \mathrm{l} \quad$ genomic DNA

$0.125 \mu \mathrm{l} \quad 10 \mathrm{mM}$ dNTP mix

$2.5 \mu \mathrm{l} \quad 5 \times$ Go-Taq DNA polymerase buffer

$0.25 \mu \mathrm{l} 100$ pmol oligonucleotide primer (GFAPCRE TG olMR1084, GFAPCRE TG OIMR1085)

$0.2 \mu \mathrm{l} \quad$ PfuS DNA polymerase

$7,2 \mu \mathrm{l} \quad$ bidistilled water

PCR reactions were run in the T3 thermocycler at the following cycling conditions:

\begin{tabular}{|c|c|c|}
\hline Temperature & Time & Step \\
\hline $98.5^{\circ} \mathrm{C}$ & $3 \mathrm{~min}$ & Initial denaturation \\
\hline $98.5^{\circ} \mathrm{C}$ & $30 \mathrm{~s}$ & Denaturation \\
\hline $65^{\circ} \mathrm{C}$ & 35 cycles & Annealing \\
\hline $72^{\circ} \mathrm{C}$ & $1 \mathrm{~min}$ & Extension \\
\hline $72^{\circ} \mathrm{C}$ & $2 \min$ & Final extension \\
\hline $4^{\circ} \mathrm{C}$ & $\infty$ & storage \\
\hline
\end{tabular}


To analyze the amplified PCR product, the samples $(10 \mu \mathrm{l})$ were loaded on an agarose gel in TAE buffer containing ethidium bromide. The cre recombinase allele was detected by a band at $100 \mathrm{bp}$.

\subsubsection{PCR for the analysis of RFX4 transcript variants in MS}

The PCR analysis to determine the specific RFX4 variant in MS lesions was performed by Andreas Junker. We received a cDNA sample from an active MS lesion from the Netherlands brain bank. About $30 \mathrm{ng}$ of the synthesized cDNA was used for each PCR reaction. The primers sequence for the specific variant 1, 2/4 and 3 are listed in Table 8.

Each PCR reaction included:

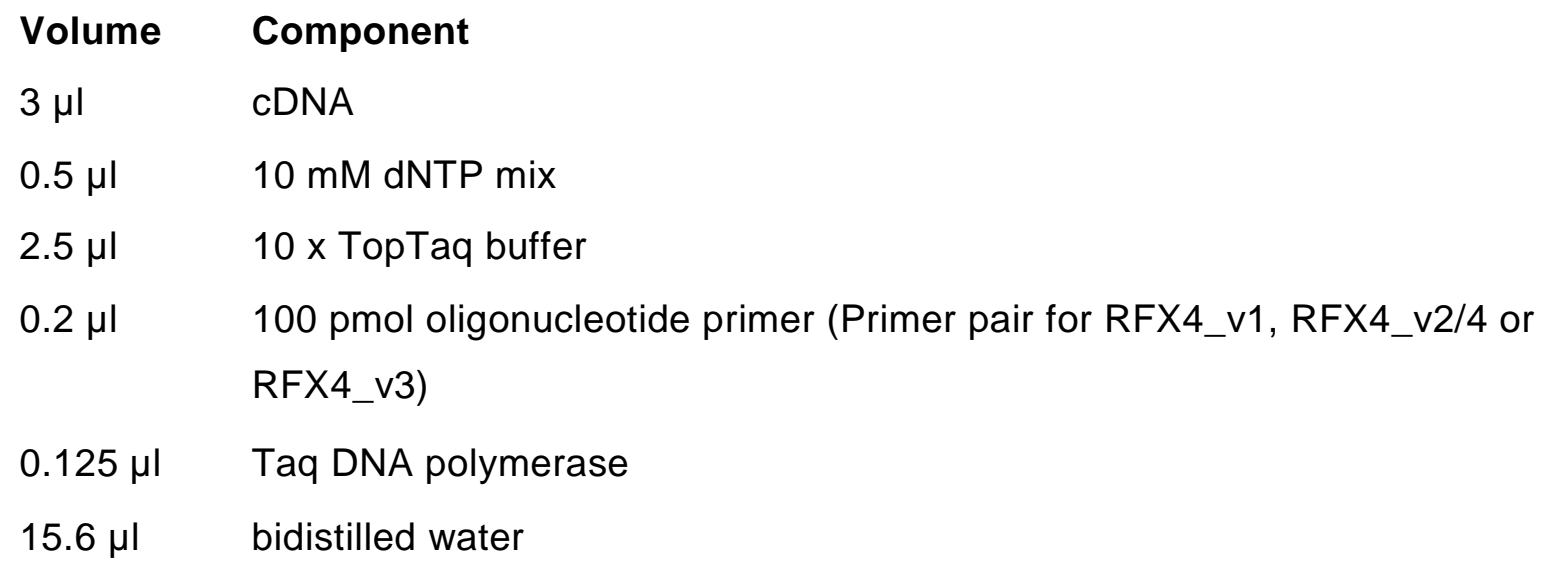

PCR reactions were run in the Eppendorf thermocycler at the following cycling conditions:

$\begin{array}{lll}\text { Temperature } & \text { Time } & \text { Step } \\ 95^{\circ} \mathrm{C} & 5 \mathrm{~min} & \begin{array}{l}\text { Initial denaturation } \\ 95^{\circ} \mathrm{C}\end{array} \\ 55^{\circ} \mathrm{C} & 1 \mathrm{~min} \\ 72^{\circ} \mathrm{C} & 30 \mathrm{~s} \\ 72^{\circ} \mathrm{C} & 45 \mathrm{~s} \\ 10^{\circ} \mathrm{C} & 10 \mathrm{~min} & \begin{array}{l}\text { Denaturation } \\ \text { Annealing }\end{array} \\ & \infty & \text { Extension } \\ & & \text { Final extension }\end{array}$

To analyze the amplified PCR product, the samples $(10 \mu \mathrm{l})$ were loaded on an agarose gel in TAE buffer containing ethidium bromide. The RFX4 variant 1 was characterized by a band at $260 \mathrm{bp}$, the RFX4 variant 2 and 4 by a band at 270 bp and RFX4 variant 3 by a band at about 500 bp. 


\subsubsection{Cuprizone mouse model}

To analyze demyelination seven to eight week old GFAP cell specific RFX4 conditional knockout mice and C57BL/6J mice were fed with the cupper chelator cuprizone in normal chow $(0.25 \%)$ for 4 weeks. To analyze remyelination the $0.25 \%$ cuprizone diet was removed after 4 weeks and mice were fed with a normal diet for either 7 or 14 days. After de- and remyelination brains of mice were prepared for analysis by histochemistry, immunohistochemistry, flow cytometry and real time quantitative PCR.

\subsubsection{Histology}

To perform histology mice were transcardially perfused with a 4\% PFA solution in PBS. Therefore mice were intraperitoneally injected with a lethal dose of a $14 \%$ chloral hydrate solution. Perfusion was performed through the left heart ventricle briefly with PBS and followed by $4 \%$ PFA solution. For post- fixation brain, liver and spleen from each mouse were removed and collected in 4\% PFA for at least 2 days. After post fixation the tissue was transferred to PBS and embedded in paraffin. Therefore brains were cut into 2-3 $\mathrm{mm}$ coronal sections, washed in water, dehydrated over night (alcohol/xylene/paraffine series) and then embedded into paraffin. For histological analysis embedded coronal brain sections were cut using a sliding microtome to obtain 1 $\mu \mathrm{m}$ thick sections. These $1 \mu \mathrm{m}$ sections were mounted onto glass slides. The tissue sections were then deparaffinized and rehydrated. For deparaffinization sections were prior incubated for one hour at $60^{\circ} \mathrm{C}$ and then transferred to xylene. The deparaffinization and rehydration steps were performed as follows:

$\begin{array}{lrl}4 x & 10 \min & x y l e n e \\ 1 x & 5 \min & x y l e n e / \text { isopropyl alcohol } \\ 2 x & 5 \mathrm{~min} & 100 \% \text { isopropyl alcohol } \\ 1 \times & 5 \mathrm{~min} & 90 \% \text { isopropyl alcohol } \\ 1 \times & 5 \mathrm{~min} & 70 \% \text { isopropyl alcohol } \\ 1 \times & 5 \mathrm{~min} & 50 \% \text { isopropyl alcohol } \\ & & \text { distilled water }\end{array}$

After histochemistry or immunohistochemistry sections were dehydrated by performing the xylene/ isopropyl series above in a reversed direction. Finally, sections were mounted with DePex mounting medium. 


\subsubsection{Luxol Fast Blue - Periodic Acid Schiff (LFB-PAS) staining}

LFB-PAS staining was performed to determine the extent of demyelination in RFX4 conditional knockout mice and $\mathrm{C} 57 \mathrm{BL} / 6 \mathrm{~J}$ mice. Therefore brain sections were deparaffinized, rehydrated to $90 \%$ isopropyl alcohol and incubated in LFB working solution over night at $60^{\circ} \mathrm{C}$. The following day sections were washed with $90 \%$ isopropyl alcohol. For the differentiation step, sections were shortly incubated in $0.05 \%$ lithium carbonate, $70 \%$ isopropyl alcohol and finally washed in distilled water to stop the differentiation. These 3 steps were repeated until only the myelin appeared deep blue. For the PAS staining sections were transferred in 1\% periodic acid for $5 \mathrm{~min}$, washed in distilled water and incubated in Schiff's reagent for $30 \mathrm{~min}$. Then the sections were washed for 20 min with tap water. To counterstain the nuclei the sections were incubated for 2 min in Mayers Hämalaun, washed in distilled water and shortly incubated in 1\% HCL-isopropyl alcohol for the differentiation. Finally the sections were shortly washed in distilled water and subsequently transferred for $10 \mathrm{~min}$ to tap water.

\subsubsection{Hematoxylin and eosin (H\&E) staining}

H\&E staining was performed to analyze the hydrocephalus and in particular the SCO of RFX4 conditional knockout mice. Therefore sections were deparaffinized, rehydrated and incubated for $8 \mathrm{~min}$ in Mayers Hämalaun. For differentiation the sections were transferred to $1 \% \mathrm{HCL}$-isopropyl alcohol, shortly washed in distilled water and transferred for $10 \mathrm{~min}$ to tap water. Then the sections were transferred to a $1 \%$ eosin solution for 1 min and finally dehydrated and mounted with DePex.

\subsubsection{Immunohistochemistry and fluorescence immunohistochemistry}

Primary and secondary antibodies used for immunohistochemistry (IHC) and fluorescence immunohistochemistry are listed in Table 6 and Table 7.

\subsubsection{Immunohistochemistry}

Many epitopes are masked in formalin fixed tissue. For de-masking epitopes sections were heated in a microwave with either $10 \mathrm{mM}$ citric acid buffer or $1 \mathrm{mM}$ Tris-EDTA buffer (5 times for $3 \mathrm{~min}$ ). The epitope retrieval buffer used for each primary antibody is listed in Table 6. After epitope retrieval the sections cooled down at RT and were washed with bidistilled water. In the next step the sections were transferred to $3 \% \mathrm{H}_{2} \mathrm{O}_{2}$ in PBS for 20 $\min$ at $4{ }^{\circ} \mathrm{C}$ to block the endogenous peroxidase. The sections were then washed with PBS and subsequently incubated with blocking buffer for at least $20 \mathrm{~min}$ to reduce unspecific antibody binding (Table 6). After these procedures the sections were prepared for primary and secondary antibody incubation. All primary antibodies were diluted in 
blocking buffer and incubated over night at $4^{\circ} \mathrm{C}$ and then washed three times with PBS. Primary antibody binding was visualized by using biotin conjugated secondary antibodies, ExtrAvidin-Peroxidase and DAB. Therefore the sections were incubated for $1 \mathrm{~h}$ with the secondary antibody diluted in blocking buffer, another $1 \mathrm{~h}$ with the ExtrAvidin-Peroxidase 1:1000 in blocking buffer and subsequently washed with PBS. Finally DAB working solution was used to visualize the antibody binding. Thereby DAB is oxidized by the peroxidase, which produces a dark brown reaction. In the double IHC for RFX4 and GFAP, GFAP was visualized with an alkaline phosphatase conjugated secondary antibody and Fast blue. The counterstain of the nuclei was performed with Mayers Hämalaun as described above.

\subsubsection{Fluorescence immunohistochemistry}

Fluorescence double IHC was performed for RFX4 and GFAP (monoclonal mouse antibody). For that purpose the sections were incubated consecutively with the primary and secondary antibodies. Between each antibody incubation step, the sections were washed for three times with PBS. The primary antibodies were visualized with cyanine Cy3-conjugated goat-anti-rabbit IgG (RFX4) and Cy2-conjugated goat-anti-mouse IgG (GFAP).

Finally sections were incubated with DAPI $(1: 10,000)$ for $10-15 \mathrm{~min}$ at RT to counterstain the nuclei.

\subsubsection{Morphometry and data aquisition}

\section{LFB-PAS stained sections and myelin proteins MBP, CNPase}

To evaluate the extent of demyelination in the corpus callosum of RFX4 conditional knockout mice and C57BL/6J mice after 1, 2, 3, 4 weeks of demyelination and after 7 or 14 days remyelination a semi quantitative score was used as follows:

$\begin{array}{ll}\text { Score } & \text { Extent of demyelination } \\ 0 & \text { No demyelination } \\ 1 & 0-33 \% \text { demyelination } \\ 2 & 33 \%-66 \% \text { demyelination } \\ 3 & >66 \% \text { demyelination }\end{array}$




\section{$\mathrm{Mac3}^{+}$microglia/ macrophages}

The density of Mac3 positive cells after 1, 2, 3 weeks cuprizone treatment and 14 days of remyelination in the corpus callosum was determined at 400x magnification with an ocular counting grid.

$\begin{array}{ll}\text { Score } & \text { Extent of microgliosis } \\ 0 & \text { few microglia } \\ 1 & 0-33 \% \text { moderate microgliosis } \\ 2 & 33 \%-66 \% \text { marked microgliosis } \\ 3 & >66 \% \text { severe microgliosis }\end{array}$

Mac3 positive cells after 1, 2, 3 weeks cuprizone treatment and 14 days of remyelination in the corpus callosum were counted at $400 x$ magnification with an ocular counting grid.

\section{$\mathrm{APP}^{+}$spheroids and glial cells}

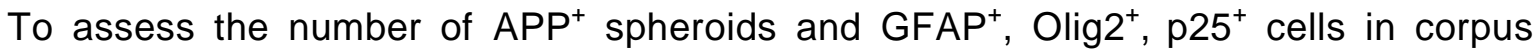
callosum of RFX4 conditional knockout mice and control mice, cells were counted at $400 \mathrm{x}$ magnification with an ocular counting grid

\section{Caspase $3^{+}$cells}

Caspase 3 positive cells were counted at 400x with an ocular counting grid after 1 week of cuprizone treatment.

\section{Double immunohistochemistry of RFX4 and GFAP in MS lesions}

Double immunohistochemistry of RFX4 and GFAP were analyzed in a chronic inactive MS lesion and in a early active lesion. The types of MS lesions were determined by Dr. Andreas Junker and Prof. Christine Stadelmann. 


\subsubsection{Isolation of astrocytes from newborn mice}

For isolation of astrocytes newborn (P0) RFX4 conditional knockout mice and C57BL/6J mice were decapitated. The skulls were immediately opened and the brains were removed and transferred into pre-warmed $\operatorname{HBSS}\left(37^{\circ} \mathrm{C}\right)$. To minimize fibroblast interference to glia cell growth meninges were removed from the brain with a forceps. After these procedures brains were collected and washed in HBSS. In a following step brains were dissected and digested with a $0.05 \%$ Trypsin EDTA solution (500 $\mu$ per brain) for $10 \mathrm{~min}$ at $37^{\circ} \mathrm{C}$. The reaction was stopped by adding $\mathrm{BME}_{\text {complete }}$ and a cell suspension was produced by pipetting up and down with a sterile pipette $(10 \mathrm{ml})$. After a centrifugation step (200 × $\mathrm{g}$ for $10 \mathrm{~min}$ ) the supernatant was exchanged by fresh $\mathrm{BME}_{\text {complete }}$ and the pellet was resuspended. The cell suspension was then transferred into a PLL coated flask and incubated at $5 \% \mathrm{CO}_{2}$ and $37^{\circ} \mathrm{C}$ in the tissue culture incubator (1 brain/ $25 \mathrm{~cm}^{2}$ flask). After 3-5 h medium was exchanged with fresh $\mathrm{BME}_{\text {complete }}$ and the primary cell culture was cultured until the cells build a 100\% confluent layer. Every 3 to 4 days medium was exchanged with fresh $\mathrm{BME}_{\text {complete. }}$

Oligodendrocytes and microglia cells were removed by vigorously agitating and washing the cells several times with PBS. The remaining astrocytes were detached with 0.05\% Trypsin-EDTA (2-3 min). To stop Trypsin-EDTA reaction BME $_{\text {complete }}$ was added to the cell suspension and then centrifuged at $200 \times \mathrm{g}$ for $10 \mathrm{~min}$. The pellet was resuspended in $\mathrm{BME}_{\text {complete }}$ and the number of astrocytes was calculated using a Neubauer counting chamber. For the quantitative PCR and the migration assay astrocytes were plated in PLL coated 24 well plates at a density of 120,000 cells per well in BME medium containing 10\% FCS and were cultured for another $48 \mathrm{~h}$.

\subsubsection{In vitro migration assay}

A cell migration assay was performed to test if astrocyte functions differ between RFX4 conditional knockout mice and C57BL/6J mice. Thereby the ability of astrocytes to migrate into a cell- free area was investigated. In a confluent astrocyte monolayer a scratch (straight line) was set with a $10 \mu \mathrm{l}$ pipet tip. To remove cellular debris the cell culture was washed with BME media. In order to always catch the same area during image acquisition, a reference point on the bottom of the 24-well plate was made with a tip marker. Images were taken after 0, 24, 33 and $48 \mathrm{~h}$. Images acquired for each well were further analyzed using the software AnalysisTM. Migration assays were performed twice with at least 3 animals (at least 3 wells/ animal) per group. 


\subsubsection{Quantitative PCR analysis}

\section{Quantitative PCR analysis of the transcript RFX4 in MS lesions}

The quantitative PCR analysis of RFX4 in MS lesions was performed by Dr. Andreas Junker. Frozen $10 \mu \mathrm{m}$ thick sections were mounted on membrane-covered polyethylene naphthalate slides (Zeiss, Jena, Germany). Parallel sections were stained with LFB to allow identification of the lesions. Tissue blocks were classified according to defined criteria: active lesions contained abundant macrophages with early (LFB and Oil Red O positive) myelin degradation products, either throughout the whole lesion or in a broad rim at the lesion edge. Inactive demyelinated lesions were sharply demarcated from the periplaque white matter, lacked a rim of microglia activation and were devoid of LFB or Oil Red O-reactive myelin degradation products in immune cells.

For the analysis of mRNA transcripts, white matter MS lesions or control white matter specimens were dissected from the slides with a scalpel and 15 sections were pooled for RNA extraction. This mRNA analysis was restricted to white matter tissue samples to limit possible confounding effects of neuronal mRNAs. The qPCR was performed on the ABI 7900 (Applied Biosystems) using the qPCR Core Kit and uracyl N-glycosylase to determine the expression level of RFX4 and other transcripts.

\section{Quantitative PCR analysis of transcripts in P0 astrocytes}

Quantitative PCR was performed from cultured PO astrocytes of RFX4 conditional knockout mice and C57BL/6J mice to characterize hydrocephalus involved genes. Therefore cultured astrocytes were washed twice with PBS, lysed with QIAzol Lysis Reagent and used for RNA isolation.

\section{Quantitative PCR analysis of transcripts in corpora callosa}

The transcript levels of RFX4, GFAP, cytokines and chemokines in the corpora callosa were measured by quantitative PCR. GFAP cell specific RFX4 conditional knockout mice and C57BL/6J mice were perfused with PBS after one week of $0.25 \%$ cuprizone treatment. The brains were removed and cut into 2-3 mm coronal sections. Brain slices with corpora callosa were briefly frozen in liquid nitrogen and the corpus callosum was immediately excised and transferred in QIAzol Lysis Reagent for the RNA isolation procedure. The experiment was repeated twice and one representative experiment is depicted in the results. 


\subsubsection{Isolation of RNA}

RNA was isolated from the corpus callosum and primary astrocyte cultures using the RNeasy Mini Kit according to the manufacturer's instructions.

\subsubsection{2 cDNA Synthesis}

The complementary DNA (cDNA) synthesis was performed with the TaqMan MicroRNA Reverse Transcription Kit and Random Hexamers. Each reaction included 300-500ng of the isolated RNA, 1 x RT buffer, 4 mM dNTPs, $10 \mu \mathrm{M}$ Random Hexamers, $1 \mathrm{U} / \mu \mathrm{l}$ RNase Inhibitor and $2.5 \mathrm{U} / \mu \mathrm{l}$ MultiScribe Reverse Transcriptase. Reverse transcription reactions were run in the Eppendorf thermocycler (Mastercycler gradient) at the following cycling conditions:

$\begin{array}{lll}\text { Temperature } & \text { Time } & \text { Step } \\ 25^{\circ} \mathrm{C} & 10 \mathrm{~min} & \text { Annealing } \\ 37^{\circ} \mathrm{C} & 120 \mathrm{~min} & \text { Reverse transcription } \\ 85^{\circ} \mathrm{C} & 5 \mathrm{~S} & \text { Inactivation of reverse } \\ & & \text { transcriptase }\end{array}$

\subsubsection{Quantitative PCR}

The quantitative PCR was performed with the qPCR Core Kit and TaqMan gene expression Assays according to the manufacturer's instructions. For the quantitative PCR reaction the cDNA was first diluted to a concentration of $3 \mathrm{ng} / \mu \mathrm{l}$. From the diluted cDNA 4 $\mu \mathrm{l}$ (12 ng) was used per qPCR reaction. Quantitative PCR reactions were run in the iQ5 ${ }^{\mathrm{TM}}$ Real Time PCR Detection System at the following cycling conditions:

\begin{tabular}{|c|c|c|}
\hline Temperature & Time & Step \\
\hline $98.5^{\circ} \mathrm{C}$ & $3 \mathrm{~min}$ & Initial denaturation \\
\hline $98.5^{\circ} \mathrm{C}$ & 40 cycles & Denaturation \\
\hline $65^{\circ} \mathrm{C}$ & $30 \mathrm{~s}\}$ & Annealing/elongation \\
\hline
\end{tabular}

The expression level of each transcript is indicated as the percentage of the housekeeping gene Gapdh. 


\subsubsection{Isolation of murine microglia cells from the corpus callosum for flow cytometry}

The isolation of phagocytes from the corpus callosum was established by and performed together with Dr. Martina Ott.

To isolate phagocytes from the corpus callosum, mice were intraperitoneally injected with a lethal dose of a $14 \%$ chloral hydrate solution and transcardially perfused with cold PBS. The brains were immediately removed and cut in 2-3 $\mathrm{mm}$ coronal sections with a razor blade. The corpus callosum was immediately excised and transferred to a $70 \mu \mathrm{m}$ cell strainer, homogenized in cold DMEM medium and centrifuged for $10 \mathrm{~min}$ at $300 \times \mathrm{g}$, $4^{\circ} \mathrm{C}$. In the next step the enzyme mix was prepared with a final concentration of $1 \mathrm{mg} / \mathrm{ml}$ Dispase II, $2.5 \mathrm{mg} / \mathrm{ml}$ Collagenase D and $1 \mathrm{mg} / \mathrm{ml}$ DNase I in DMEM medium (2 $\mathrm{ml} /$ corpus callosum). Each corpus callosum pellet was resuspended in $2 \mathrm{ml}$ of the enzyme mix and pre-incubated for $10 \mathrm{~min}$ at $37^{\circ} \mathrm{C}$. The incubation was continued with rotation for

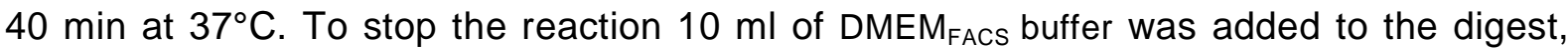
mixed and centrifuged for $10 \mathrm{~min}$ at $300 \times \mathrm{g}, 20^{\circ} \mathrm{C}$. The pellet was then washed by resuspending the cell suspension in $10 \mathrm{ml} \mathrm{DMEM}_{\mathrm{FACS}}$ buffer and spinning $10 \mathrm{~min}$ at $300 \times \mathrm{g}$, $20^{\circ} \mathrm{C}$.

During the centrifugation step the Percoll gradient was prepared. Therefore a $38 \%$ percoll solution containing $2.8 \mathrm{ml}$ percoll and $1.2 \mathrm{ml} \mathrm{DMEM}_{\mathrm{FACS}}$ buffer and a $70 \%$ percoll solution containing $1.9 \mathrm{ml}$ percoll and $3.1 \mathrm{ml}$ DMEM$_{\mathrm{FACS}}$ buffer was prepared for each corpus callosum. The $70 \%$ percoll solution was transferred to a $15 \mathrm{ml}$ canonical tube (5 $\mathrm{ml}$ per corpus callosum).

After the centrifugation the supernatant was discarded and the pellet was resuspended in the $38 \%$ percoll solution ( $4 \mathrm{ml}$ per corpus callosum) and transferred to the $15 \mathrm{ml}$ canonical tube by slowly pipetting the $4 \mathrm{ml} 38 \%$ percoll solution on top of the $70 \%$ percoll solution. The gradient was centrifuged for $25 \mathrm{~min}$ at $500 \times \mathrm{g}, 20^{\circ} \mathrm{C}$. The centrifuge stopped with no brake so that the interphase was not disturbed. From the $70 \%-38 \%$ interphase $2.0-2.5 \mathrm{ml}$ was collected into a clean $15 \mathrm{ml}$ canonical tube. To wash the cells $10 \mathrm{ml}$ FACS buffer was added, mixed and centrifuged for $10 \mathrm{~min}$ at $300 \times \mathrm{g}, 4^{\circ} \mathrm{C}$. The cell pellet was then resuspended in FACS buffer and used for the FACS staining.

\subsubsection{FACS staining of phagocytes}

After the gradient, cells from each animal were resuspended in FACS buffer $(200 \mu \mathrm{l} /$ FACS antibody), distributed on a 96 well plate and centrifuged for $5 \mathrm{~min}$ at $300 \times \mathrm{g}$ and $4^{\circ} \mathrm{C}$. After the washing step the cell pellets were resuspended in $100 \mu$ l blocking buffer and incubated for $10 \mathrm{~min}$ at $4^{\circ} \mathrm{C}$. The blocking buffer included anti-CD16/CD32 antibody diluted 1:100 in FACS buffer. To prepare the fluorochrome-labeled antibody mix, 
antibodies were diluted 1:100 in FACS buffer. From the antibody mix $100 \mu \mathrm{l}$ were added to each well to obtain a final dilution of 1:200. The cells were then incubated for another $20 \mathrm{~min}$ at $4^{\circ} \mathrm{C}$. After these procedure cells were centrifuged ( $5 \mathrm{~min}, 300 \times \mathrm{g}, 4^{\circ} \mathrm{C}$ ), and washed with $100 \mu \mathrm{l}$ FACS buffer. Finally cells were resuspended in $200 \mu \mathrm{l}$ FACS buffer and analyzed by flow cytometry.

\subsubsection{Flow cytometry analysis}

Microglia cell sorting and flow cytometry analysis were performed by Sabrina Becker of the Flow cytometry core facility at the University Medical Center Goettingen.

\subsubsection{Statistical analysis}

All statistics were calculated using the GraphPad Prism5.01 software. The data were first tested for normal distribution with the Kolmogorov-Smirnov test with Dallal-WilkinsonLilliefor $p$-value. For two experimental groups, unpaired t test was performed for parametric values, while the Mann-Whitney test was performed for non-parametric values. For comparison of more than 2 groups, one way ANOVA with Bonferroni post test was used for parametric data, while the Kruskal-Wallis test with Dunn's post test was carried out for non-parametric data. Statistical significance was defined by $p<0.05$. 
$3 \quad$ Results

\subsection{Part 1: Characterization of the GFAP cell specific RFX4 conditional knockout mice}

\subsubsection{Hydrocephalus and mortality of RFX4 conditional knockout mice}
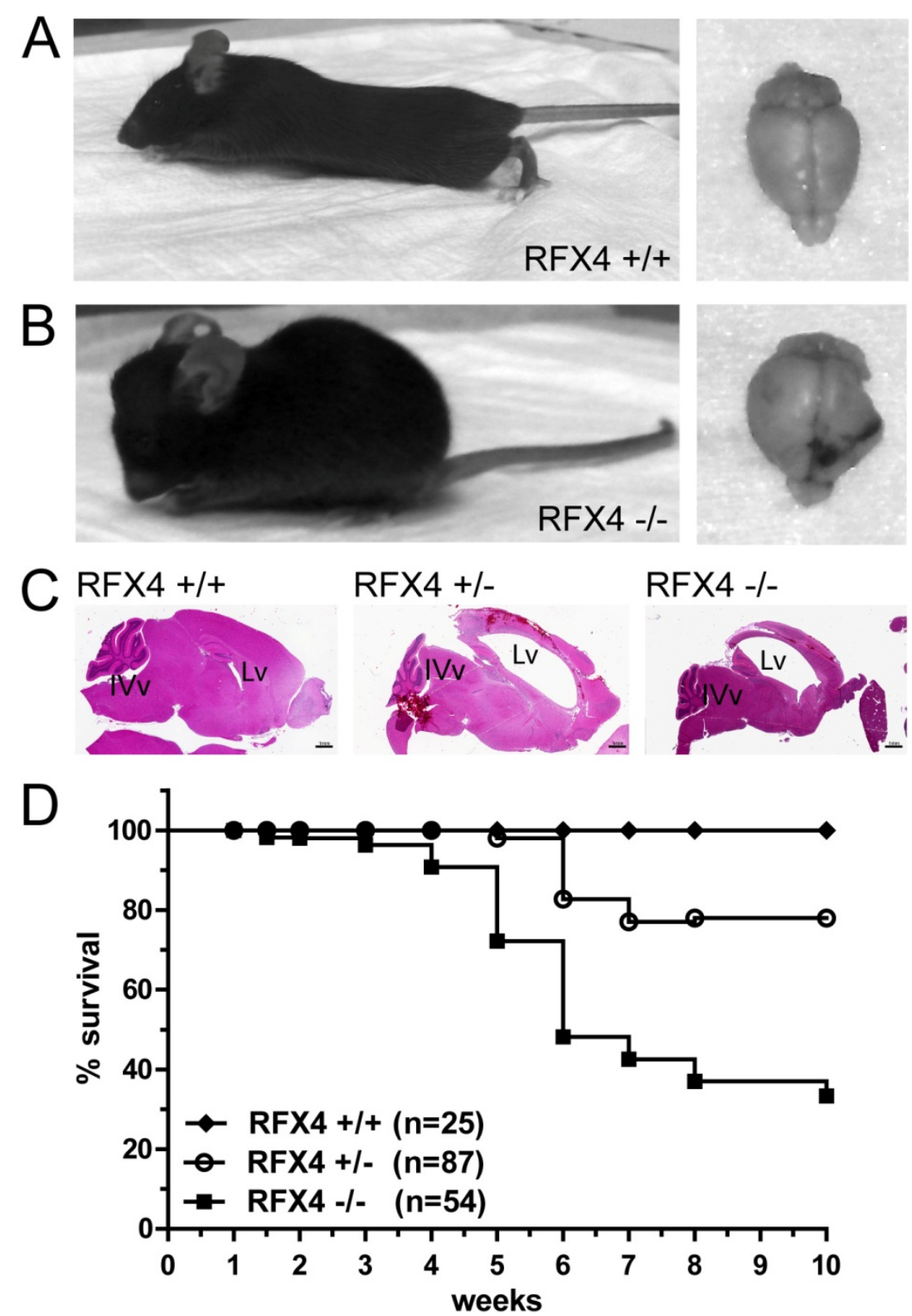

Figure 2: Hydrocephalus and mortality of GFAP cell specific RFX4 conditional knockout mice. (A-B) Appearance of wild type (A) and mutant (B) mice at the age of 5 weeks. (C) H\&E stained sagittal sections from RFX4 +/+ mice, RFX4 +/- and RFX4 -/- mice with extensive dilatation of the lateral ventricle. (D) Kaplan-Meier survival curve of RFX4 +/- mice , RFX4 -/- mice and RFX4 +/+ mice (littermate control mice). Lv, lateral ventricle, IVv, fourth ventricle. Scale bar $=1 \mathrm{~mm}$

To generate GFAP cell specific RFX4 conditional knockout mice, GFAP cre mice, expressing the Cre recombinase under control of the GFAP regulatory sequence (promoter), were crossed with RFX4 LoxP (floxed) mice. GFAP cell specific RFX4 
conditional knockout mice were born at the expected Mendelian ratio, were normal in size and appearance at birth. Several of the heterozygous mice and a large percentage of the homozygous mice exhibited head swelling (Figure $2 \mathrm{~A}, \mathrm{~B}$ ) followed by neurological deficits like convulsions and loss of balance, that ended in death in young adulthood. Heterozygous mice died at an age of 6 weeks and most RFX4 homozygous mice died between 4 to 6 weeks as shown in the Kaplan-Meier survival curve (Figure 2 D). The mortality rate at an age of 6 weeks was about $20 \%$ for RFX $4+/$ - mice, whereas RFX4 -/mice died with a rate of about $50 \%$ at the same age. This implies a gene dosage effect. Mice which did not develop a hydrocephalus had a normal phenotype. Histological examination of the brains of symptomatic adult mice manifested severe hydrocephalus, with extensive dilatation of the lateral ventricles, but no distention of the fourth ventricle (Figure $2 \mathrm{C}$ ). Investigation of transgenic mice at the time of birth or few days after birth showed that the hydrocephalus was already present, indicating that the hydrocephalus is congenital (Figure 3). This suggested a congenital blockage in the aqueduct of Sylvius as has been reported for RFX4_v3 transgenic mice generated by Blackshear and colleagues, which leads to disruption of RFX4_v3 (Blackshear et al., 2003).
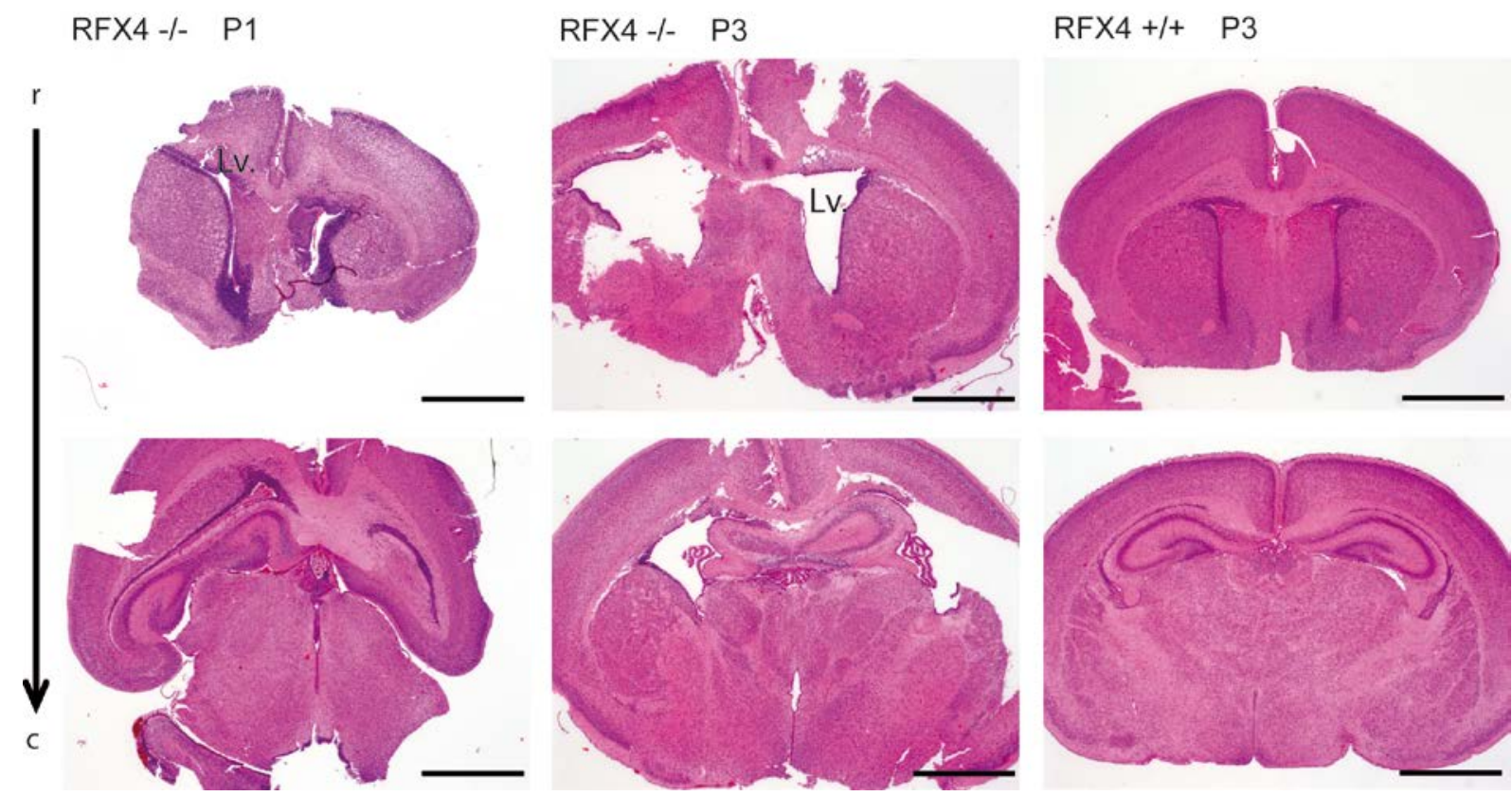

Figure 3: Hydrocephalus of newborn RFX4 conditional knockout mice.

H\&E stained sections from rostral ( $r$ ) to caudal (c), from both P1 and P3 RFX4 homozygous conditional knockout mice and P3 wild type animals. Lv, lateral ventricle. Scale bar $=1 \mathrm{~mm}$

\subsubsection{Hydrocephalus in RFX4 conditional knockout mice is associated with abnormalities of the subcommissural organ}

The primary cause of a congenital hydrocephalus is stenosis or narrowing of the Sylvian aqueduct in the midbrain. The origin of the stenosis is not well understood, but it 
has been reported that maldevelopment and resulting dysfunction of the SCO is involved in the cause of aqueductal stenosis (Overholser et al., 1954; Vio et al., 2000). The SCO is a small gland that secretes glycoproteins of high molecular mass into the ventricles to form Reissner's fiber (Nualart et al., 1991; Rodríguez et al., 1998). The SCO is situated in the dorsocaudal region of the third ventricle at the entrance of the Sylvian aqueduct (Rodríguez et al., 1998).

Part of the thesis was to explain the reason for the hydrocephalus formation in RFX4 conditional knockout mice. For that purpose, the severity of the hydrocephalus in symptomatic adult RFX4 conditional knockout mice was assessed in serial sections from caudal to rostral. In addition, the presence of the SCO and the severity of SCO hypoplasia was investigated (Table 14). 10 homozygous and 8 heterozygous mice with hydrocephalus were analyzed.

Table 14: Severity of the hydrocephalus and SCO hypoplasia in RFX4 conditional knockout mice

\begin{tabular}{lllllllll} 
Strain & $\begin{array}{l}\text { RFX4 } \\
\text { deficient } \\
\text { mice } \\
\text { analyzed }\end{array}$ & $\begin{array}{l}\text { Severity of } \\
\text { hydrocephalus }\end{array}$ & & $\begin{array}{l}\text { Presence } \\
\text { of SCO }\end{array}$ & $\begin{array}{l}\text { Severity of Sco } \\
\text { hypoplasia }\end{array}$ \\
\hline & Moderate & Marked & Severe & Moderate & Marked & Severe \\
\hline RFX4 +l- & 8 & 4 & 2 & 2 & 8 & 4 & 3 & 1 \\
\hline RFX4 -I- & 10 & 3 & 3 & 4 & 10 & 2 & 4 & 4 \\
\hline
\end{tabular}

Sections from caudal to rostral were studied from RFX4 heterozygous and homozygous conditional knockout mice with hydrocephalus. The severity of the hydrocephalus, the presence of the SCO and the severity of the SCO hypoplasia were assessed. Two representative animals for each genotype are presented in Figure 4. 


\subsubsection{GFAP cell specific RFX4 conditional knockout mice suffer from} congenital hydrocephalus of variable severity
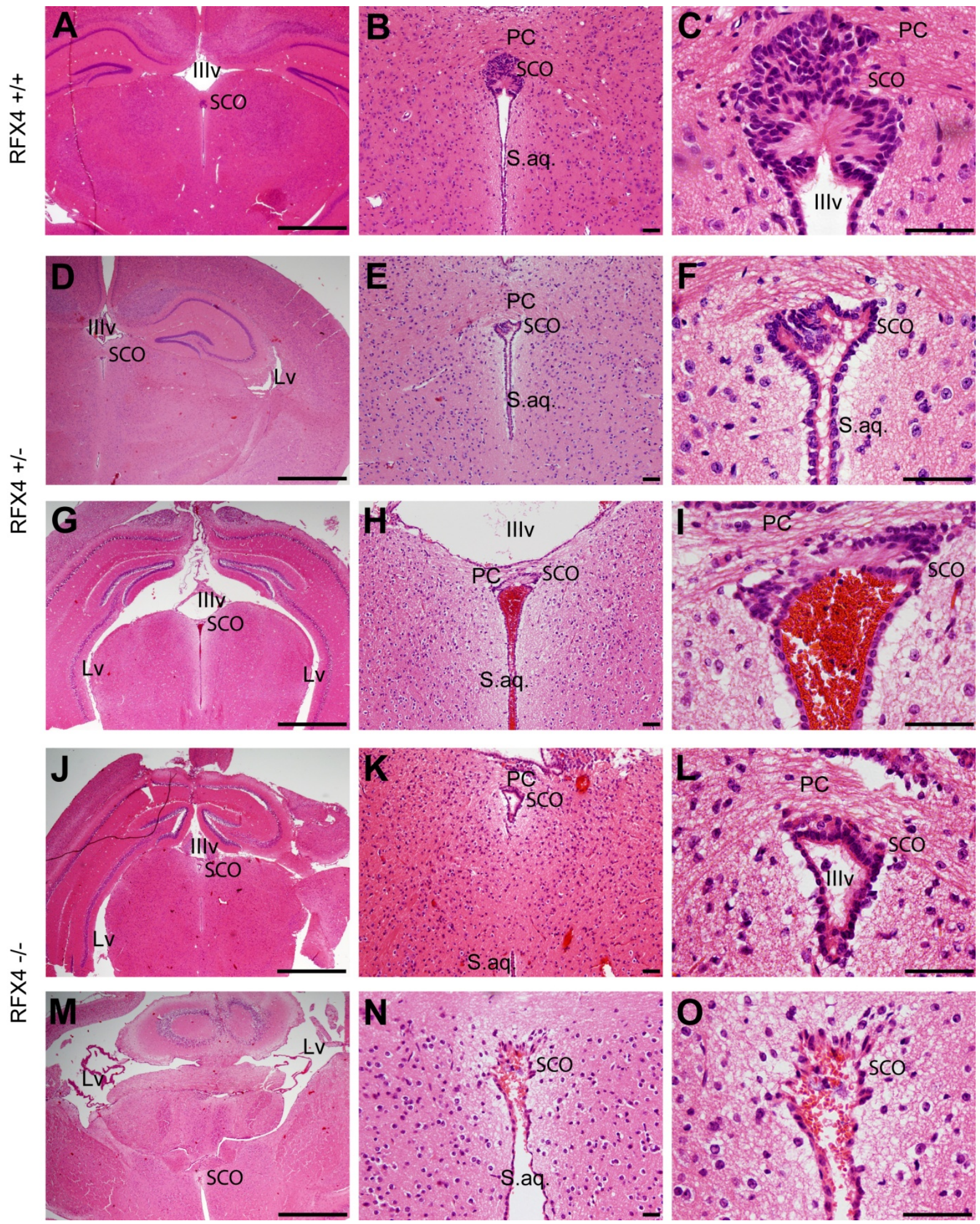

Figure 4: GFAP cell specific RFX4 conditional mice suffer from congenital hydrocephalus of variable severity accompanied by SCO hypoplasia.

$\mathrm{H} \& \mathrm{E}$ histochemistry from wild type and RFX4 deficient mice with hydrocephalus of variable severity and SCO hypoplasia of each presented animal. $(A, B, C)$ reflects a wild type mouse, (D,E,F) a RFX4 +/mouse with a moderate hydrocephalus and marked SCO hypoplasia, (G,H,I) a RFX4 +/- mouse with 
marked hydrocephalus and moderate SCO hypoplasia, (J,K,L) a RFX4 -/- mouse with marked hydrocephalus and severe SCO hypoplasia and $(\mathrm{M}, \mathrm{N}, \mathrm{O})$ shows a RFX4 -/- mouse with severe hydrocephalus with severe SCO hypoplasia. SCO, subcommissural organ, Lv, lateral ventricle, IIIv, third ventricle, S.aq., Sylvian aqueduct, $P C$, posterior commissure. Scale bar $=(A, D, G ; J ; M) 1 \mathrm{~mm}$, (B,C,E,F,H,I,K,L,N,O) $50 \mu \mathrm{m}$

All analyzed mice developed a hydrocephalus, while the severity of the hydrocephalus was variable (Figure 4). Most of the RFX4 +/- mice had a moderate to marked hydrocephalus, while the majority of the homozygous animals showed hydrocephalus of a marked to severe degree. This may indicate an effect of gene dosage on the severity of hydrocephalus. In the most marked to severe cases, hydrocephalus was characterized by extensive dilatation of the lateral and third ventricles (Figure $4 \mathrm{G}, \mathrm{M}$ ), while less severe cases showed only a mild dilatation of the lateral ventricles and no distention of the third ventricle (Figure $4 \mathrm{D}$ ).

\subsubsection{Subcommissural organ hypoplasia}

The SCO was present in all analyzed animals. However all animals studied exhibited different degrees of SCO hypoplasia. The degree of SCO hypoplasia correlated well with the severity of the hydrocephalus as is shown in Table 14 and Figure $4 \mathrm{G}-\mathrm{I}$ and M-O. In wild type animals, ependymal cells of the SCO are elongated and their apical poles protrude in the direction of the third ventricle, where they secrete glycoproteins (Figure 4 A-C). In contrast RFX4 conditional knockout mice showed a reduced number of ependymal cells (Figure $4 \mathrm{~F}, \mathrm{I}, \mathrm{L}, \mathrm{O}$ ). Remaining cells in the posterior commissure were flattened (Figure $4 \mathrm{I}, \mathrm{L}$ ), arranged in a disordered fashion (Figure $4 \mathrm{~F}, \mathrm{O}$ ) and their apical borders no longer protruded into the lateral ventricle (Figure $4 \mathrm{~F} ; \mathrm{I}$; L; O). The highly specialized ependymocytes of the SCO are either absent or inappropriately differentiated in RFX4 conditional knockout mice. 


\subsubsection{Dysfunction of the SCO}
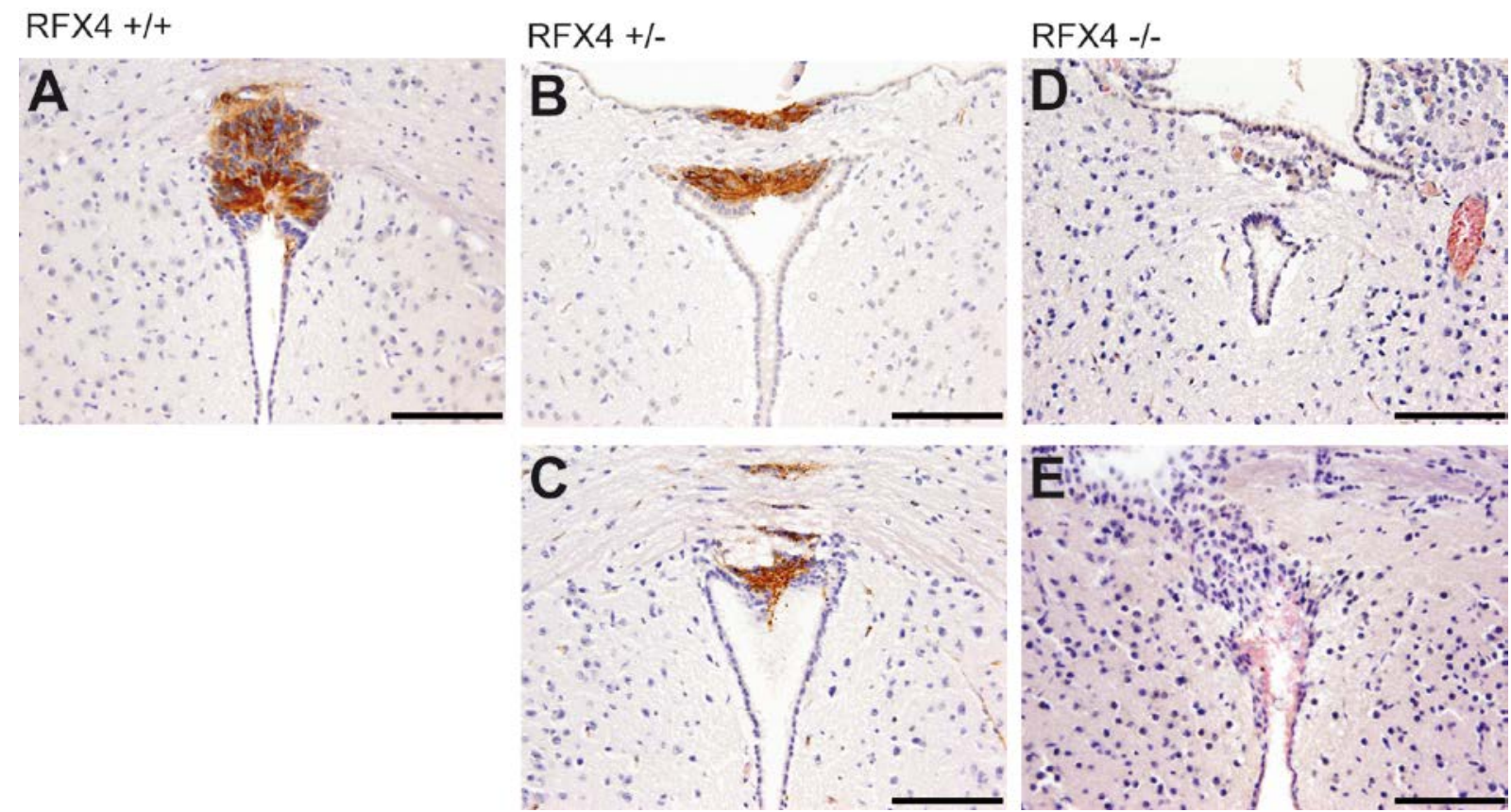

Figure 5: Expression of SCO RF glycoprotein is deregulated in RFX4 conditional knockout animals.

IHC with an antibody specific for RF glycoproteins (AFRU) of young adult brain sections of wild type and RFX4 deficient mice. (A) represents a wild type mouse, (B-C) a RFX4 +/- mouse and (D-E) shows a RFX4 -/- mouse. Scale bar $=50 \mu \mathrm{m}$

Due to maldevelopment of the SCO in RFX4 deficient mice I assumed that the SCO may be dysfunctional. A well developed SCO secretes glycoproteins of high molecular mass (Nualart et al., 1991). The majority of the glycoproteins are released in the ventricle where they aggregate to form a threadlike structure, known as Reissner's fiber (Sterba and Wolf, 1969). To investigate whether the SCO of RFX4 conditional knockout mice is functional, adult brain section were stained with an antibody specific for RF glycoproteins (AFRU).

In wild type mice strong RF glycoprotein expression was restricted to the SCO. This conspicuous glycoprotein expression was lacking in RFX4 conditionally deficient mice. It was replaced by a lower level of SCO glycoprotein production in RFX4 +/- or was completely absent in several cases of RFX4 -/- mice (Figure 5).

\subsubsection{Loss of developmental factors impairs SCO formation and function}

The SCO is derived from neuroepithelial cells that line the lumen of the dorsocaudal aspect of the diencephalon (Huh et al., 2009). These neuroepithelial precursors of the SCO differentiate into specialized secretory ependymocytes in response to the induction of Wnt and Bmp signals (Huh et al., 2009). Components of the Wnt and Bmp signaling 
pathway were reported to be potential targets for RFX4_v3 in E10.5 brains (Blackshear et al., 2003; Zhang et al., 2006a). Due to the observed hypoplasia of the SCO of RFX4 conditional knockout mice I assumed a similar deregulation of the Wnt and Bmp signaling pathways in our conditional knockout mouse. In order to test this hypothesis I cultured astrocytes of PO mice and characterized them morphologically. In a second step I isolated RNA from PO astrocytes and measured the expression levels of components involved in the Wnt and Bmp signaling pathway by qPCR. A selection of critical genes for early brain development, based on a microarray of heads from RFX4_v3 -/- mice at E10.5, were measured by qPCR (Zhang et al., 2006a).

\subsubsection{Characterization of RFX4 deficient P0 astrocytes in vitro}

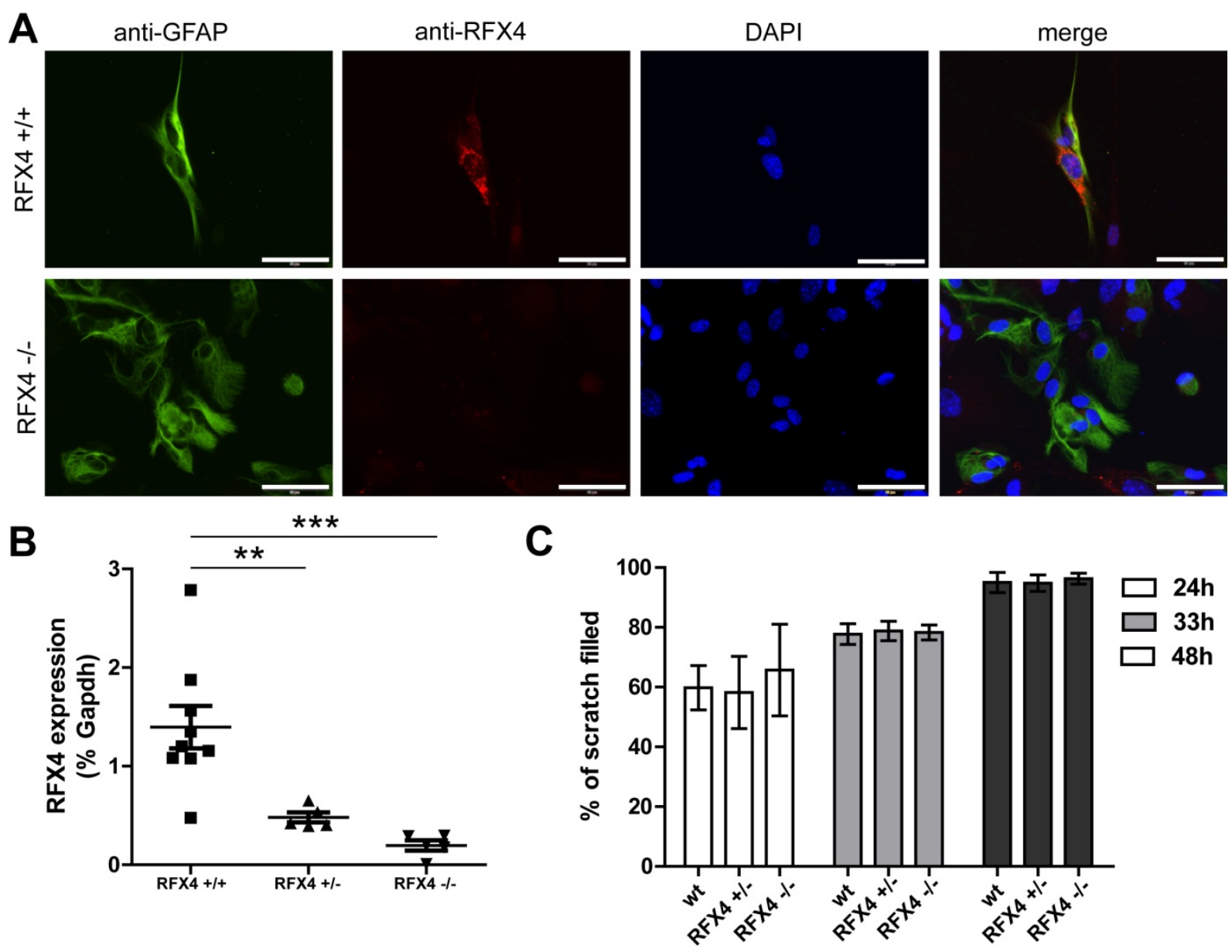

Figure 6: Characterization of RFX4 deficient P0 astrocytes in vitro.

(A) Representative IHC for GFAP and RFX4 of wild type and RFX4 deficient astrocytes. (B) Quantitative real time PCR for RFX4 from P0 astrocytes obtained from individual wild type and RFX4 deficient animals ( ${ }^{*} p<0.01,{ }^{* \star *} p<0.001$, one way ANOVA with Bonferroni post test). (C) Migration scratch assay of wild type, RFX4 +/- and RFX4 -/- mice. A scratch was made in an astrocyte monolayer from wild type, RFX4 +/- and RFX4 -/- mice. The ability to migrate was compared after $24 \mathrm{~h}$, $33 \mathrm{~h}$ and 48h. $(n(w t)=4 ; n(R F X 4+/-)=3 ; n(R F X 4-/-)=5)(C)$. Data are presented as mean \pm SEM. Scale bar $=50 \mu \mathrm{m}$ 
Astrocytes were isolated and cultured from GFAP cell specific RFX4 conditional knockout mice and wild type mice at time of birth. To measure the expression level of RFX4 RNA was isolated from astrocytes of RFX4 +/-, RFX4 -/- and wild type mice. Quantitative real time PCR showed a significant reduction of RFX4 transcripts in PO astrocytes obtained from RFX4 conditional knockout mice compared to wild type mice (Figure $6 \mathrm{~B}$ ). The transcript level of RFX4 showed a reduction of about 3- fold for RFX4 +/- and about 7- fold for RFX4 -/- compared to wild type astrocytes. Immunohistochemistry for GFAP showed characteristically shaped astrocytes comparable to the wild type. Immunohistochemistry for GFAP and RFX4 of RFX4 conditional knockout mice and wild type astrocytes illustrates expression of RFX4 in the nucleus, perinucleus and in the cytoplasm of wild type astrocytes. In astrocytes obtained from RFX4 -/- mice reduced expression was observed (Figure 6 A).

A migration assay was performed to test whether RFX4 deficiency affects astrocyte functions. A scratch was made in an astrocyte cell monolayer from wild type, RFX4 +/and RFX4 -/- mice and the ability to migrate was compared after $24 \mathrm{~h}, 33 \mathrm{~h}$, and $48 \mathrm{~h}$. The ability to migrate into a cell free area was similar for RFX4 conditional knockout mice and wild type animals. In vitro astrocytes from RFX4 conditional knockout mice did not display obvious morphological and functional deficits. 


\subsubsection{Regulation of hydrocephalus associated genes in RFX4 conditional knockout astrocytes.}

A Wnt signaling
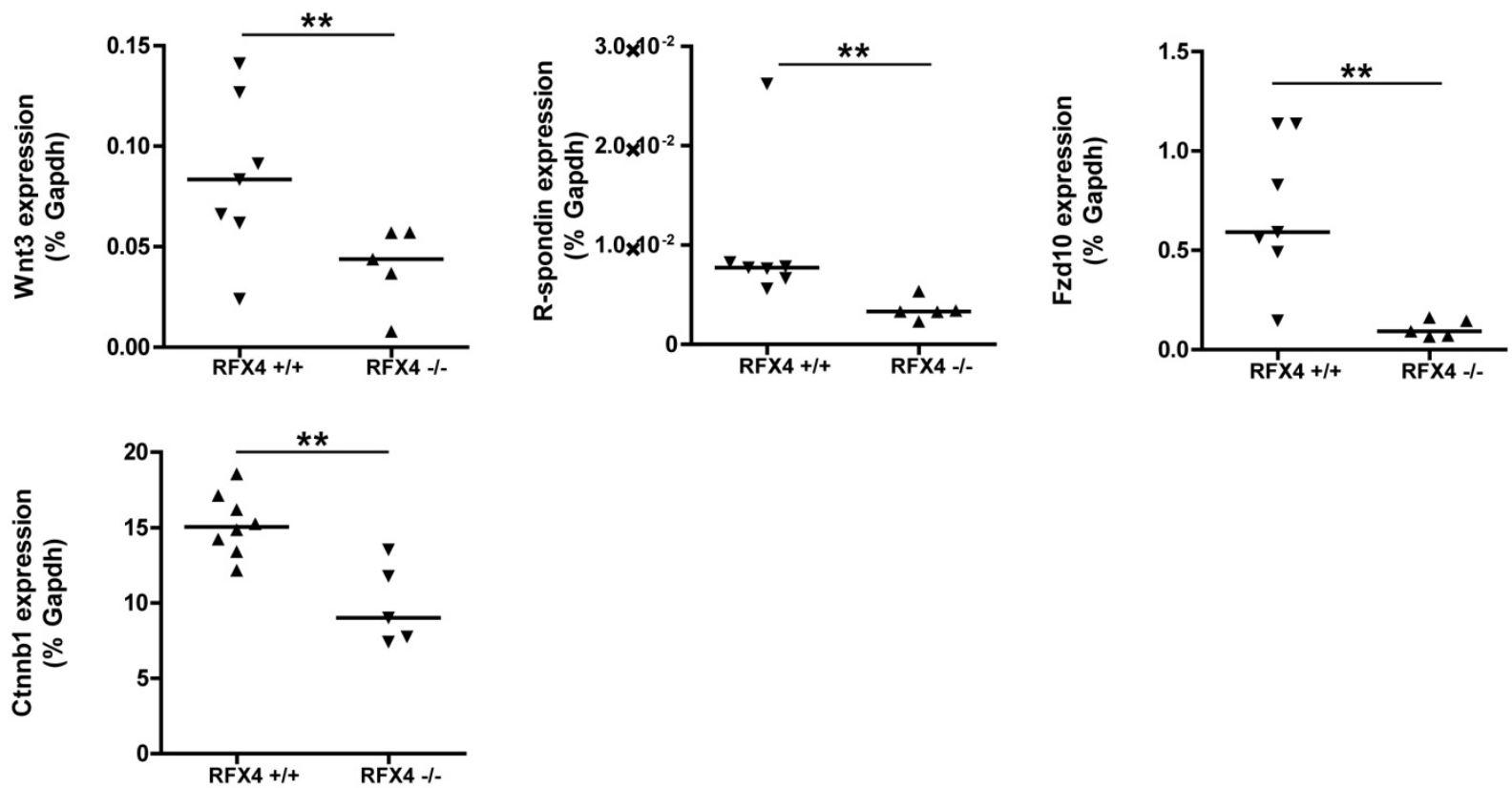

B Genes regulated in early development
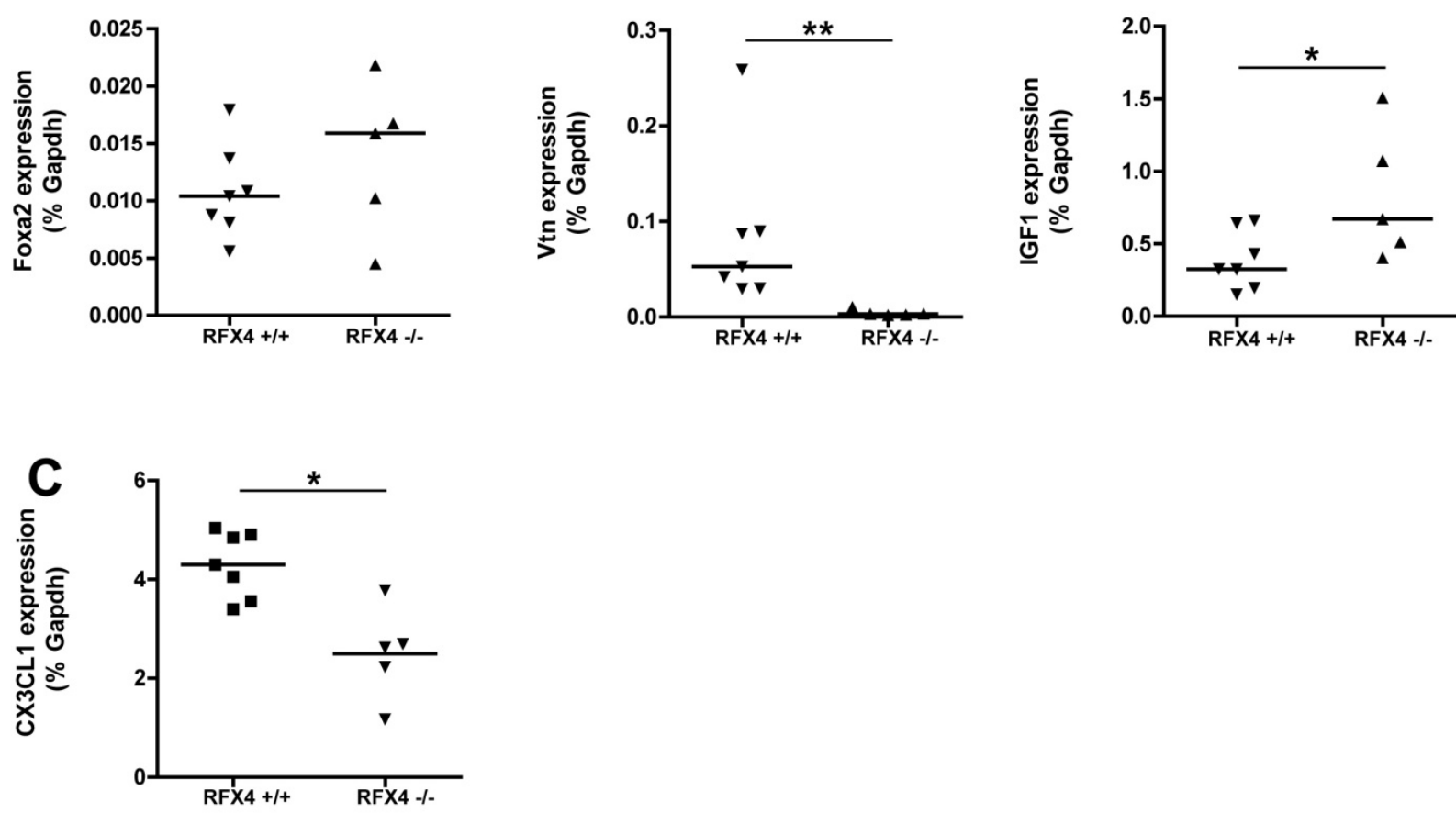

Figure 7: Regulation of hydrocephalus associated genes in RFX4 conditional knockout astrocytes.

Based on Zhang and colleagues 2006 selected genes relevant for the development of congenital hydrocephalus (A) and factors critical for early brain development (B) were analyzed by quantitative real time PCR. (A-B) qPCR was performed from mRNA of cultured P0 astrocytes of RFX4 conditional 
knockout mice and control mice ( $p>0.05,{ }^{*} p<0.05,{ }^{\star \star} p<0.01$, Mann Whitney test). The mean of each group is indicated.

Based on Zhang and colleagues, 2006, which identified potential target genes of the brain specific variant 3 of RFX4, the expression levels of selected genes were measured by qPCR of RNA from P0 astrocytes isolated from the brains of RFX4 -/- and wild type mice. For the Wnt pathway Wnt3, Wnt3a, R-spondin, Frizzeled 10 (Fzd10) and ß-catenin were measured. For the Bmp signaling pathway Msx3 and cytochrome P450 26A1 (Cyp26a) were studied. Furthermore, other factors critical for early brain development Foxa2, vitronectin (Vtn), IGF1 and CX3CL1 were measured. The expression levels of Wnt3a, Msx3 and Cyp26a were not measurable (too low) in P0 astrocytes with a raw CT $>35$. Hence a difference between wild type and RFX4 conditional knockout animals was not measurable. However mRNA levels of all other transcripts were detectable and were evaluated.

\subsection{Transcript changes of Wnt signaling}

The Wnt signaling pathway is an evolutionary conserved pathway that regulates important aspects of cell fate determination, cell migration, cell polarity, neural patterning and embryonic development (Komiya and Habas, 2014). Zhang and colleagues studied potential target genes in RFX4_v3 deficient mice at embryonic stage 10.5 and identified four genes which belonged to the Wnt signaling pathway. Their transcript levels were dramatically down- regulated in heads from RFX4_v3 -/- mice. The levels of the secreted Wnt3 and Wnt3a glycoproteins were decreased by approximately 5- and 16- fold (Zhang et al., 2006a). In our RFX4 conditional knockout mice the level of Wnt3 was reduced by approximately 3- fold at the time of birth (Figure $7 \mathrm{~A}$ ). The expression level of Wnt3a was not detectable at this time point.

Fzd10 belongs to the frizzled gene family and was decreased by 2.4 - fold in E10.5 brains of RFX4_v3 -/- mice (Zhang et al., 2006a). In this study it was found to be reduced 6- fold compared to astrocytes of wild type mice (Figure 7 A). R-spondin was decreased by 1.5- fold at E10.5 (Zhang et al., 2006a). The factor is a transmembrane protein that acts as activator of the canonical/wnt B-catenin signaling pathway (de Lau, Wim B M et al., 2012). The gene was reduced by about two fold in RFX4 conditional knockout astrocytes compared to wild type (Figure $7 \mathrm{~A}$ ). B-catenin was not detected by Zhang and colleagues in E10.5 heads, but it has been reported that it acts as an intracellular signal transducer in the Wnt signaling pathway (Clevers and Nusse, 2012). Further, the gene was found to be crucial for proper midbrain development, and mutation of ß-catenin led to hydrocephalus (Ohtoshi, 2008). In conditional RFX4 knockout mice, ß-catenin was significantly down-regulated by about 1.5- fold (Figure $7 \mathrm{~A}$ ). 


\subsection{Expression of factors critical for early brain development and neuronal regulators}

During neuroblastoma cell differentiation Foxa2 up-regulates Vtn expression (Shimizu et al., 2002). Both genes were found to be down-regulated in E10.5 RFX4_v3 -/- heads. Vtn was decreased by about 1.7- fold and Foxa2 by about 1.5- fold (Zhang et al., 2006a). In RFX4 conditional mice mRNA levels of Vtn were still significantly reduced whereas transcript levels of the transcription factor Foxa2 were not significantly changed at the time of birth (Figure $7 \mathrm{~B}$ ). CX3CL1 has been reported as direct target gene, because RFX4_v3 binds to the CX3CL1 promoter (Zhang et al., 2006a). CX3CL1 is a chemokine that was decreased by approximately 3- fold in E10.5 heads of RFX4_v3 -/- mice (Zhang et al., 2006a; Zhang et al., 2008). RFX4 conditional knockout animals showed a reduction by approximately 1.7- fold at P0 (Figure 7 B). IGF1 was increased by two fold in E10.5 heads of RFX4_v3 deficient mice (Zhang et al., 2006a). Insulin like growth factor has been shown to play role in regulating neural development including neurogenesis and myelination (Beck et al., 1995). Conditional RFX4 knockout mice did also up-regulate IGF1 compared to wild type mice by about two fold (Figure 7 B). 


\subsection{Part 2: The role of astrocytic RFX4_v3 in demyelination}

\subsubsection{RFX4 in MS}

\subsubsection{RFX4 is produced by astrocytes and up-regulated in MS lesions}

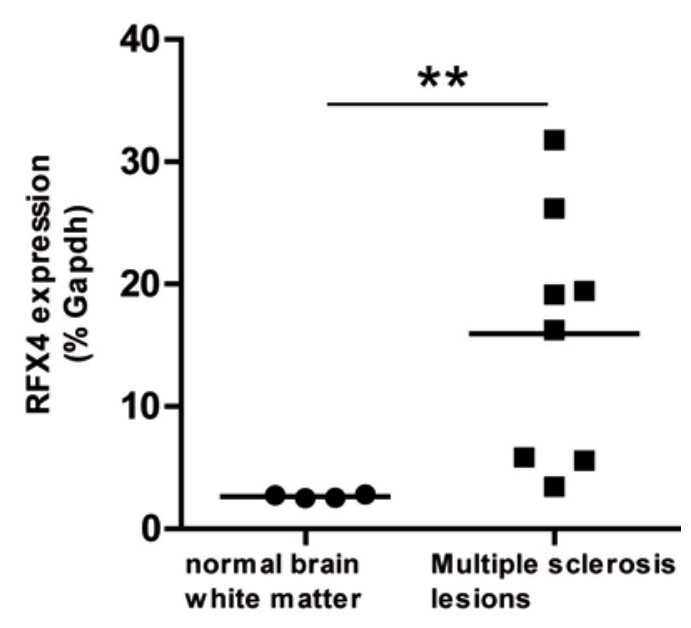

Figure 8: RFX4 is up-regulated in MS lesions.

RFX4 expression levels in individual tissue samples were determined by quantitative PCR. The mean of each group is indicated. ( ${ }^{* \star} p<0.01$, Mann Whitney $U$ test).

MS is an inflammatory, demyelinating, neurodegenerative disorder of the CNS. A wealth of data describe that astrocytes play an active and possibly dual role in CNS inflammatory diseases such as MS. On the one side they have the ability to increase immune responses and inhibit myelin repair, on the other side they can also be protective and limit CNS inflammation while supporting oligodendrocyte and axonal regeneration (Nair et al., 2008). To determine astrocyte functions during inflammatory demyelination in MS lesions, Andreas Junker did a quantitative PCR study of MS lesions, together with normal brain white matter as control. 96 astrocyte specific genes, which were identified by Cahoy and colleagues 2008, were measured (Cahoy et al., 2008).

This approach revealed RFX4 as a highly up-regulated factor in MS lesions. The transcript level of RFX4 was about 6-fold higher in MS lesions compared to normal brain white matter $\left({ }^{*} \mathrm{p}<0.01\right)$ (Figure 8 ). The expression level of RFX4 in normal brain white matter was clearly detectable with a mean of $2.5 \%$ Gapdh.

To determine astrocytic RFX4 expression in MS lesions double IHC for RFX4 and GFAP was performed on an actively demyelinating MS lesion, chronic inactive MS lesion and on normal appearing white matter (NAWM) as control (Figure 9). MS lesions were characterized using LFB-PAS histochemistry (Figure 9 A-E). RFX4 was detectable in NAWM and in both active and chronic inactive MS lesions (Figure 9). The transcription factor showed nuclear localization in various cell types including immune cells and glia cells of NAWM (Figure 9 A,F,K) and MS lesions of different activity status (Figure 9 G-J). 
Double labeling for GFAP and RFX4 exhibited RFX4-expressing astrocytes in both acute (Figure $9 \mathrm{G}$ ) and chronic MS lesions (Figure $9 \mathrm{H}-\mathrm{J}$ ). In the presented active lesion RFX4 positive astrocytes were detected in the perivascular area and at the lesion border (Figure $9 \mathrm{G}$ ). In the chronic inactive lesion RFX4 positive astrocytes were located in the lesion center and more prominently at the lesion border (Figure $9 \mathrm{I}, \mathrm{J}$ ). 


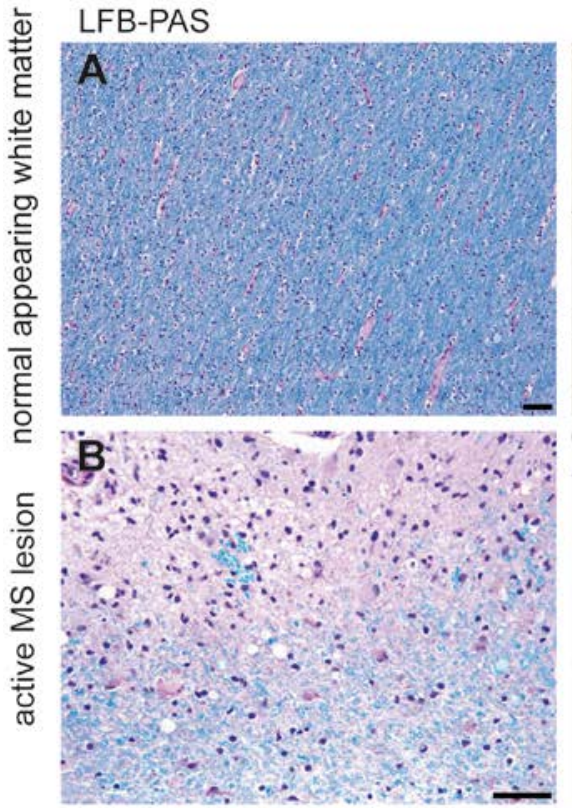

\section{anti-RFX4/ anti-GFAP}

anti-RFX4/ anti-GFAP
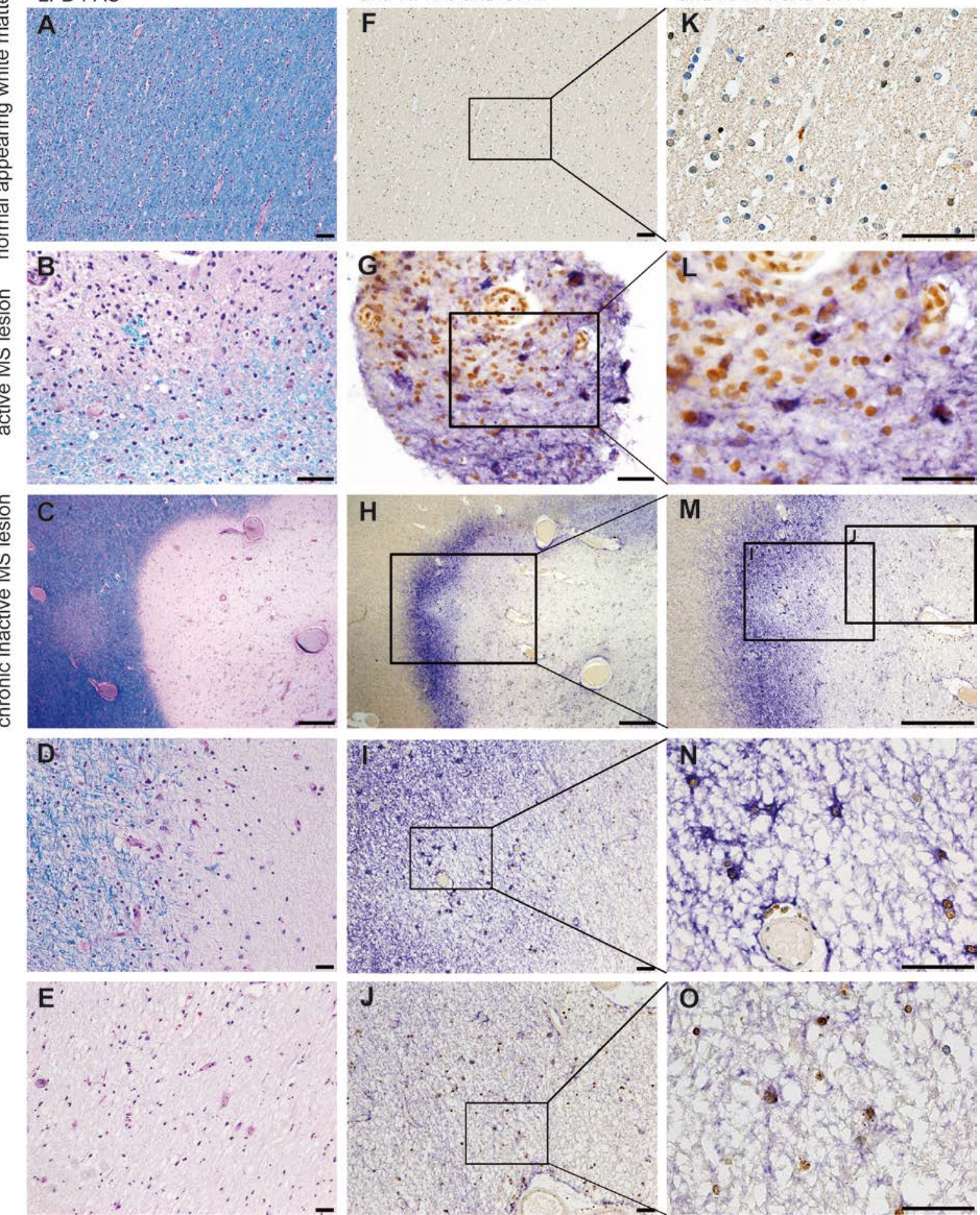

Figure 9: RFX4 is expressed by astrocytes and up-regulated in MS.

Histochemistry and immunohistochemistry using sections of normal appearing white matter, an active MS lesion and a chronic inactive MS lesion as indicated. (A-E) Histochemistry using LFB-PAS. (F-J) Double immunohistochemistry using anti-RFX4 (brown) and anti-GFAP (blue) antibodies. (K-O) Closeup of anti-RFX4 and anti-GFAP IHC shown in F-J. Scale bars $=(\mathbf{C}, \mathbf{H}, \mathbf{M}) 500 \mu m$; $(A, F, K, B, G, L, D, I, N, E, J, O) 50 \mu m$ 


\subsubsection{RFX4 variant 3 is expressed in MS lesions}

A Genomic structure

B

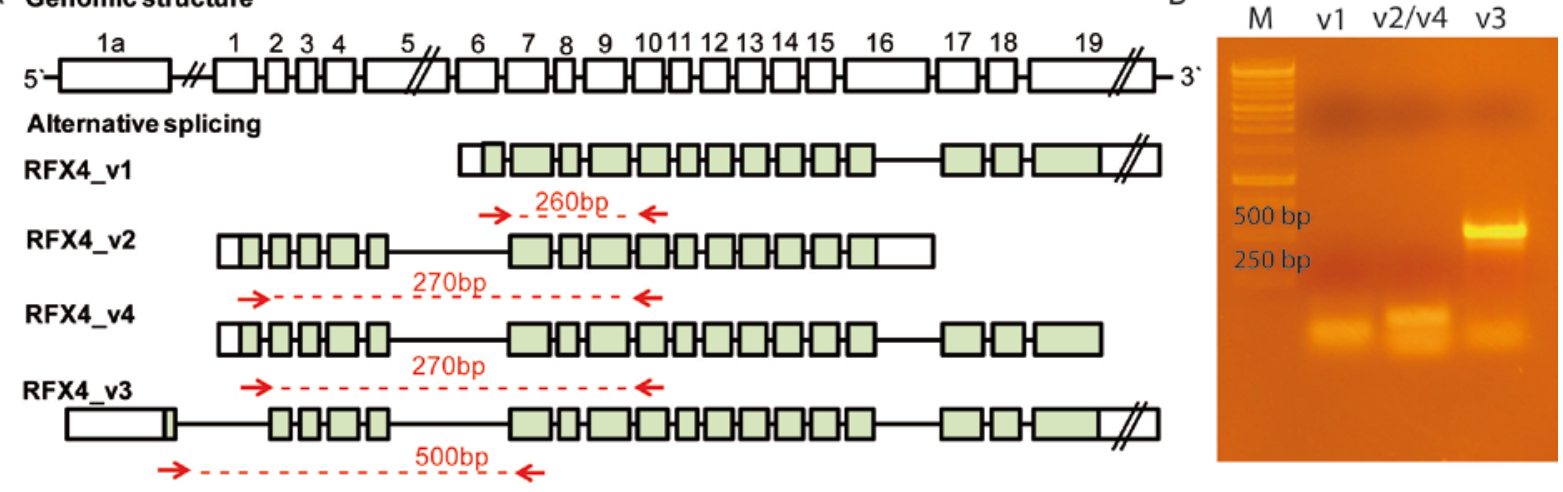

Figure 10: Only RFX4_v3 is expressed in MS lesions.

Expression of the transcript variants RFX4_v1,_v2, _v3, _v4 in MS lesions was measured by RT PCR using variant-specific primer pairs for v1, v2/v4 and v3. (A) Schematic representation of RFX4 genomic structure and its main splice variants. The exons are depicted as boxes, and open reading frames are shown as green boxes. Primer pairs used for RT PCR are presented as red arrows and red dashed lines. Expected lengths of RT PCR products are indicated. (B) Agarose gel electrophoresis of RT PCR reactions. Gel was stained with ethidium bromide. M=DNA size marker

In humans four main alternatively spliced variants of RFX4 have been described in normal tissue, which include RFX4_v1,_v2, _v3 and _v4 (Figure 10 A). In the testis variants v1, v2 and v4 are expressed and in the brain only RFX4_v3 has been detected (Matsushita et al., 2005).

To investigate the expression of transcript variants RFX4_v1, _v2, _v3 and _v4 in MS lesions RT PCR was performed using published variant specific primer pairs (Matsushita et al., 2005). In MS lesions no RFX4_v1, _v2 and_v4 mRNA was detectable. RT PCR detected only variant 3 with the expected length of 500 base pairs in MS lesions (Figure $10 \mathrm{~B})$. RFX4_v3, was identified by the binding of the forward primer to exon 1 which is unique for RFX4_v3. 


\subsubsection{Role of astrocytic RFX4_v3 during de- and remyelination in the cuprizone mouse model}

Pathologically, MS is characterized by the presence of areas of demyelination and inflammation in the white and gray matter of the CNS. Demyelination denominates a selective loss of myelin with relative axonal preservation. The natural response to demyelination is remyelination, the process of rebuilding the myelin sheaths including the generation of new oligodendrocytes. Unfortunately extensive remyelination of MS lesions is only rarely observed (Franklin, Robin J M and ffrench-Constant, 2008; Stadelmann and Brück, 2008; Kierdorf et al., 2013). Astrocytes are implicated to play a crucial role in regulating de- and remyelination in MS (Nair et al., 2008).

Regarding the finding of astrocytic RFX4_v3 in MS lesions, I assumed a possible role for the factor in the process of de- and remyelination. Therefore, the cuprizone mouse model was used to investigate the role of astrocytic RFX4_v3 during de- and remyelination.

Demyelination was studied using 7 to 8 week old GFAP cell-specific RFX4_v3 conditional knockout mice without hydrocephalus and control mice (C57BI/6). Both were treated for 4 weeks with a $0.25 \%$ cuprizone diet. To analyze remyelination cuprizone was removed from the diet after 4 weeks and mice were kept on a cuprizone-free diet for another 7 or 14 days. 
3.2.2.1 Cuprizone intoxication for 4 weeks induces strong demyelination in the corpus callosum of RFX4_v3 conditional knockout mice

A

Cuprizone

week 0

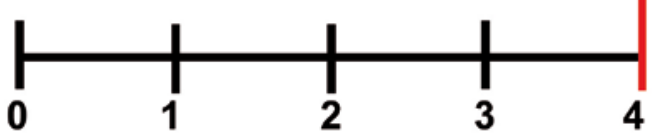

B LFB-PAS corpus callosum

$$
\text { corpus callosum medial }
$$

corpus callosum lateral
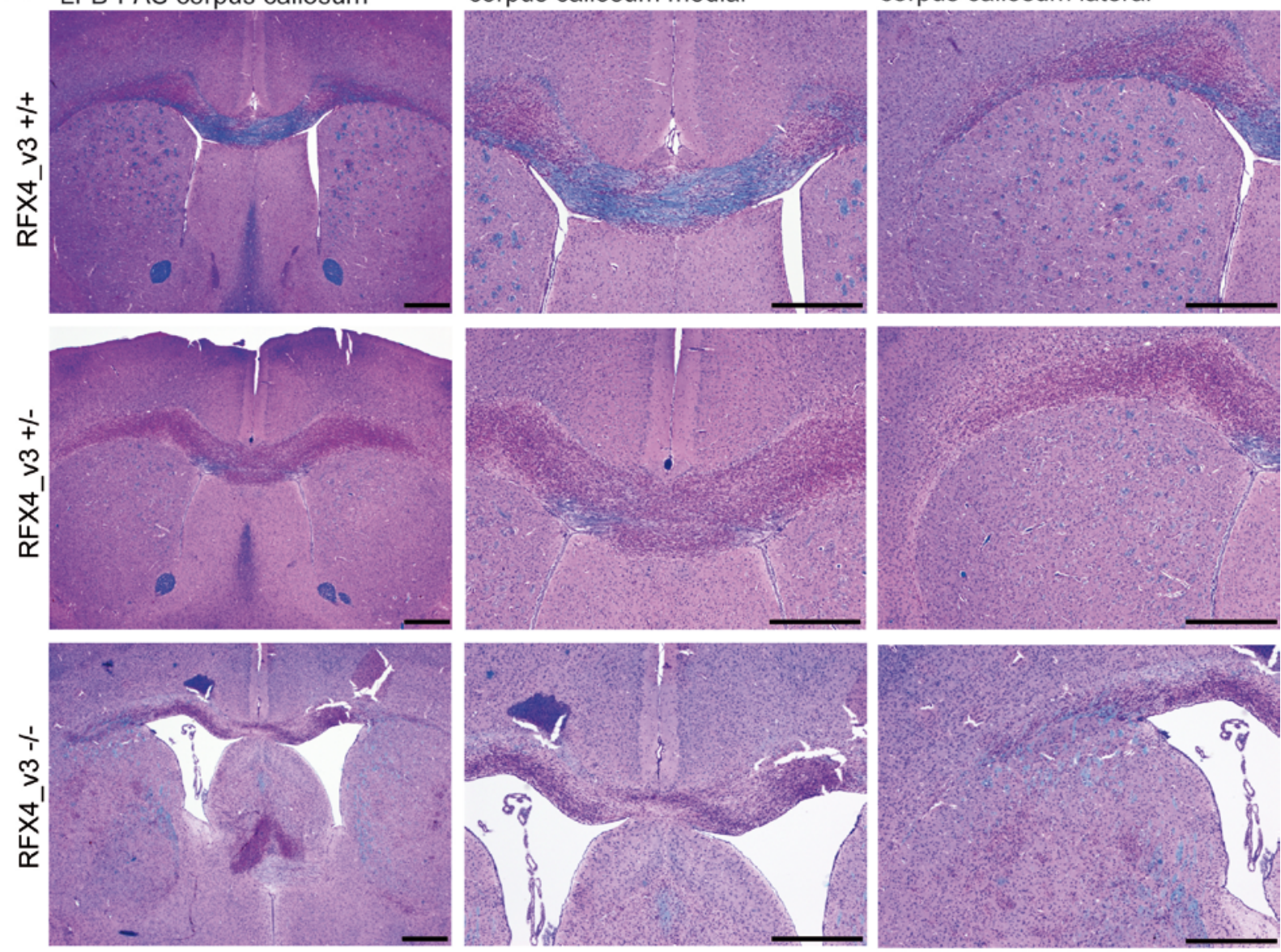

C corpus callosum medial

D corpus callosum lateral
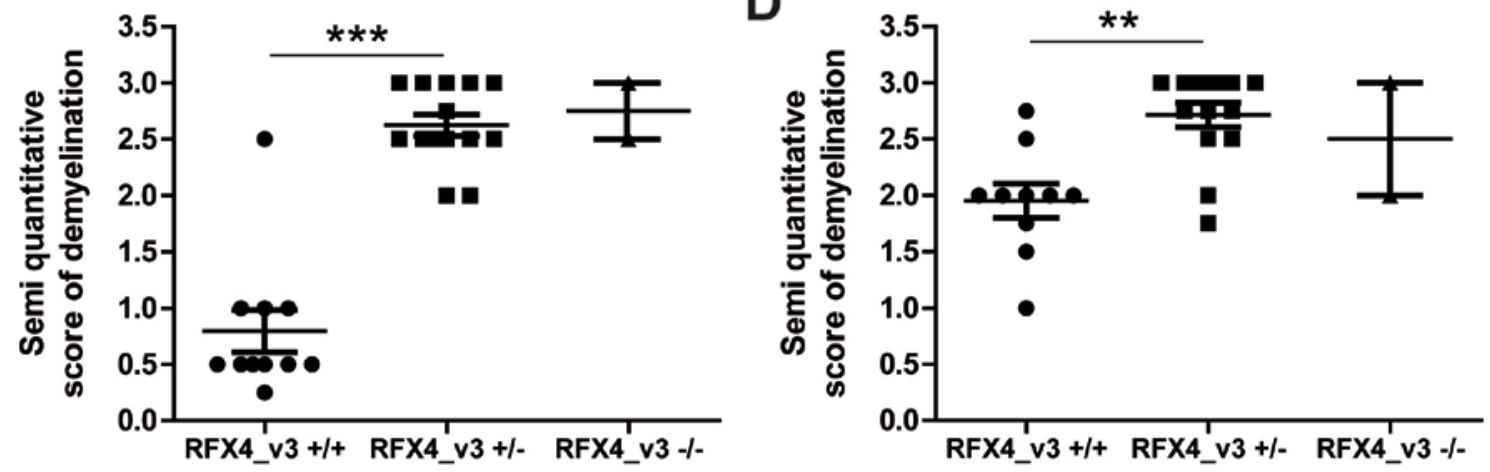

Figure 11: Cuprizone feeding for 4 weeks induces strong demyelination in the corpus callosum of RFX4_v3 conditional knockout mice.

RFX4_v3 +/+, RFX4_v3 +/- and RFX4_v3 -/- mice were treated for 4 weeks with a 0.25\% cuprizone diet. (A) Schematic representation of cuprizone treatment. (B) LFB-PAS staining of representative sections from RFX4_v3 +/+, RFX4_v3 +/- and RFX4_v3 -/- mice after 4 weeks of cuprizone ingestion. 
(C-D) Quantification of demyelination by scoring LFB-PAS staining in the medial and lateral corpus callosum. A score of 0 represents complete myelination and a score of 3 represents complete

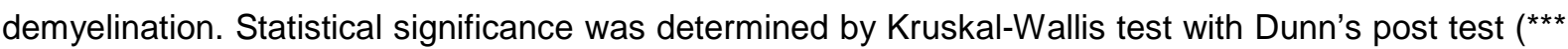
$\left.p<0,001,{ }^{\star *} p<0,01\right)$. Data are presented as mean \pm SEM. Scale bar $=500 \mu \mathrm{m}$

A corpus callosum medial

B corpus callosum lateral
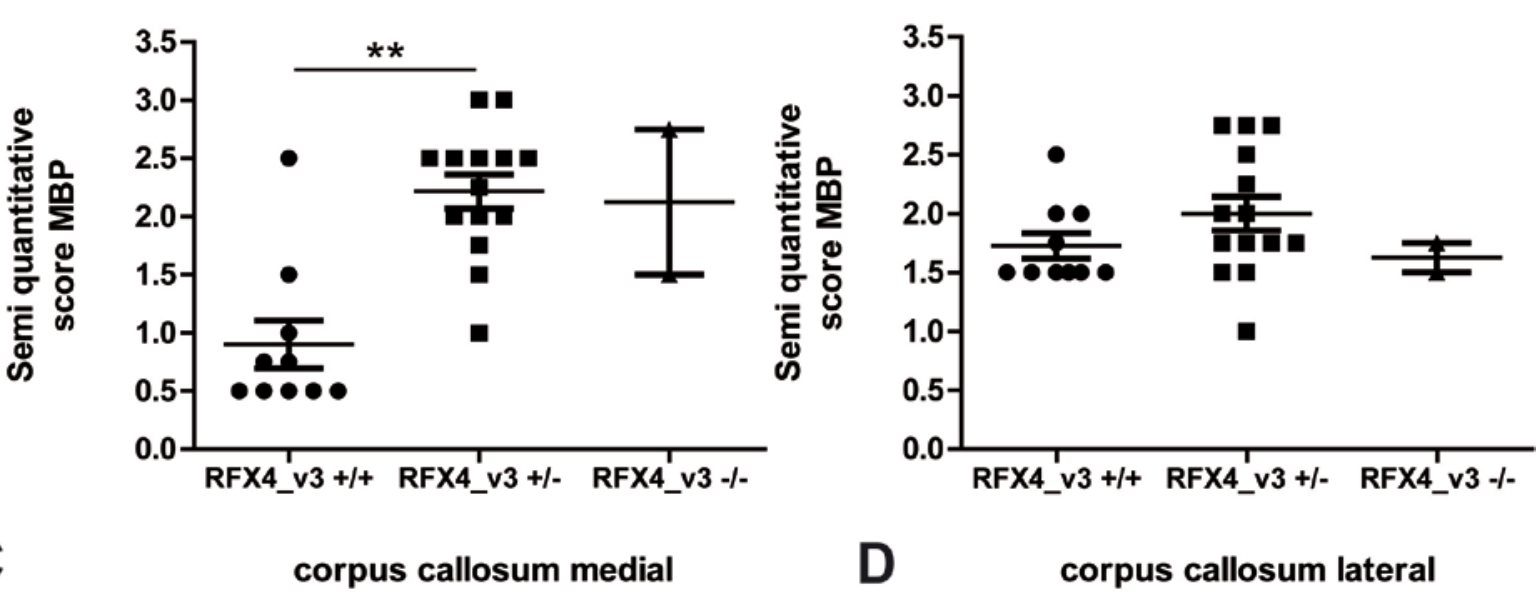

C corpus callosum medial
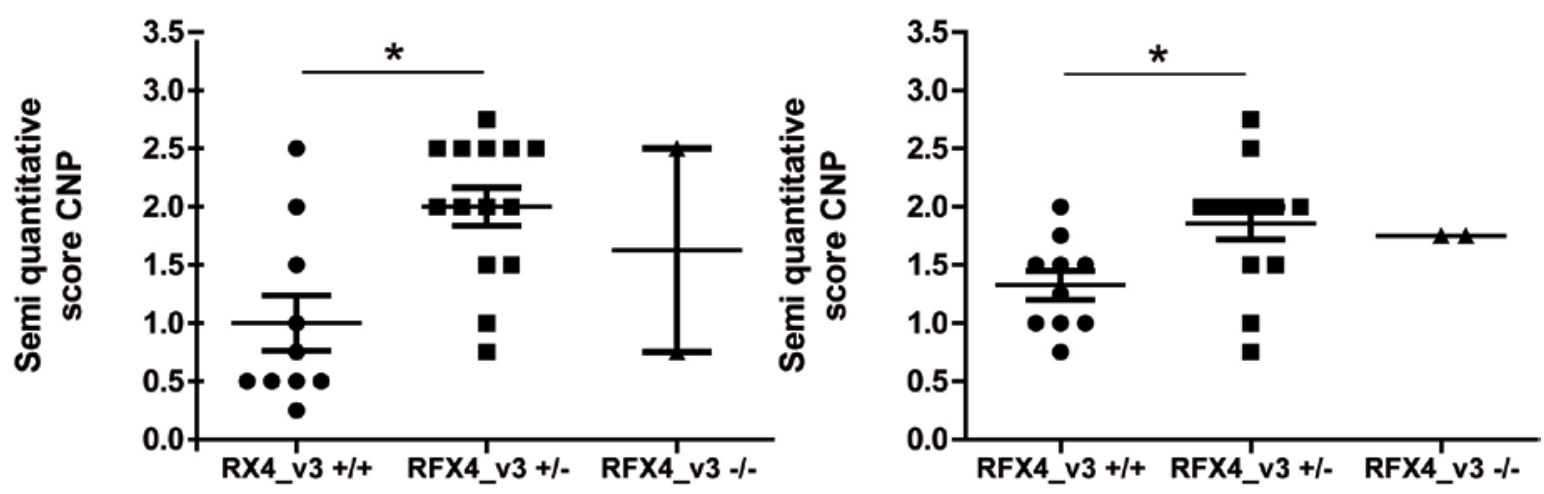

Figure 12: Cuprizone-induced demyelination correlates with reduced myelin proteins in the corpus callosum of RFX4 deficient animals.

Brain sections from RFX4_v3 +/+, RFX4_v3 +/- and RFX4_v3 -/- mice were stained for myelin proteins MBP and CNPase. Extent of demyelination was quantified by scoring (A-B) MBP and (C-D) CNPase in corpus callosum medial $(\mathbf{A}, \mathbf{C})$ and corpus callosum lateral $(\mathbf{B}, \mathbf{D})$. Score of 0 represents complete myelination and score of 3 represents complete demyelination. Statistical significance was determined by Kruskal-Wallis test with Dunn's post test $\left({ }^{*} p<0.05,{ }^{* *} p<0.01\right)$. Data are presented as mean \pm SEM.

Brain sections from RFX4_v3 +/+, RFX4_v3 +/- and RFX4_v3 -/- mice were examined and scored for demyelination by LFB-PAS staining (Figure 11). Thereby, a score of 0 represents complete myelination and a score of 3 represents complete demyelination in the corpus callosum. The corpus callosum of RFX4_v3 deficient mice was almost completely demyelinated after cuprizone treatment, while the myelin of wild type animals appeared to be still intact especially in the medial part of the corpus callosum after week 4 of cuprizone intoxication. No difference was observed between RFX4_v3 +/- and 
RFX4_v3 -/- mice. Compared to wild type animals RFX4_v3 +/- conditional knockout animals showed a significantly increased demyelination in the medial (score medial RFX4_v3: +/- $2.6 \pm 0.1$ vs RFX4_v3 +/+: $0.8 \pm 0.2, * \star * p<0.001$ ) and lateral (score lateral RFX4_v3: $+/-2.7 \pm 0.1$ vs. $1.9 \pm 0.2{ }^{* *} p<0.01$ ) part of the corpus callosum on LFB-PAS stained sections.

In addition, enhanced demyelination after week 4 of cuprizone diet was confirmed by the reduced presence of myelin proteins in the corpus callosum of RFX4 deficient animals (Figure 12). The extent of demyelination was evaluated by scoring sections from RFX4_v3 +/+, RFX4_v3 +/- and RFX4_v3 -/- mice stained for myelin proteins MBP and CNPase. MBP as well as CNPase were significantly diminished mainly in the medial part of the corpus callosum of RFX4_v3 +/- compared to control mice (MBP score medial 2.2 \pm 0.14 vs. $0.9 \pm 0.2,{ }^{*} p<0.01$ ) (CNPase score medial $2 \pm 0.16$, score medial $1 \pm 0.23$, $\left.{ }^{*} \mathrm{p}<0.05\right)$ (Figure $\left.12 \mathrm{~A}, \mathrm{C}\right)$. 


\subsection{Increased axonal damage in RFX4 deficient mice}
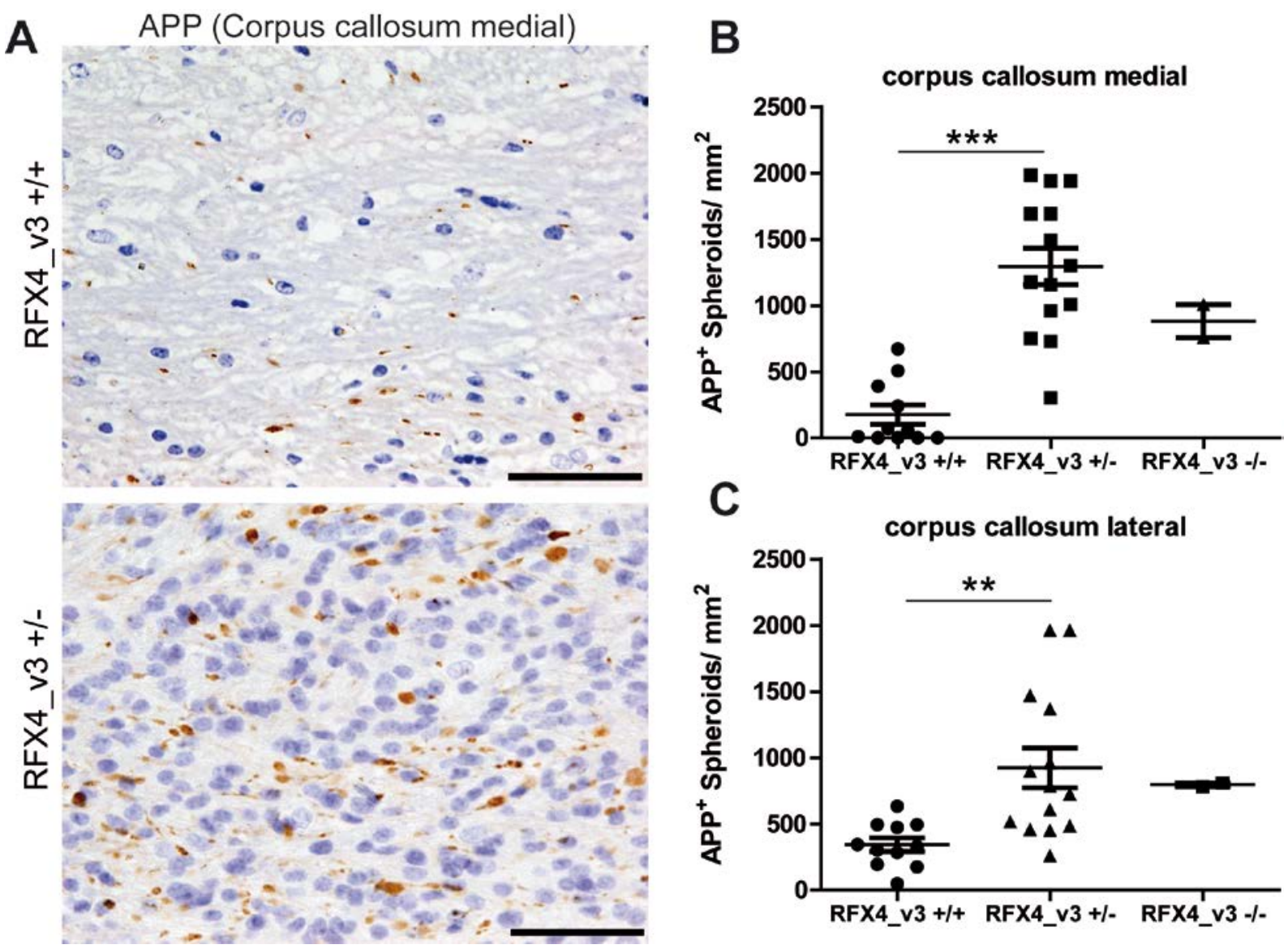

Figure 13: Increased demyelination was accompanied by enhanced acute axonal damage in the corpus callosum of RFX4_v3 deficient mice.

(A) APP IHC of brain sections from wild type and RFX4_V3 conditional knockout mice after cuprizone treatment. A representative medial section from corpus callosum is shown. (B-C) Quantification of APP positive spheroids in corpus callosum medial (B) and lateral (C) sections of indicated mice after 4 weeks of cuprizone intoxification ( ${ }^{\star *} p<0,01,{ }^{* \star} p<0,001$, Kruskal-Wallis test with Dunn's post test). Data are presented as mean \pm SEM. Scale bar $=50 \mu \mathrm{m}$

Substantial demyelination, as seen in the corpus callosum of RFX4_v3 deficient mice after cuprizone treatment, can lead to axonal damage. I thus investigated axonal transport disturbance in corpus callosum sections from wild type and RFX4_v3 conditional knockout animals by IHC for APP (Figure 13).

APP IHC revealed increased numbers of APP positive spheroids in RFX4_v3 deficient mice after week 4 of cuprizone intoxication. The quantitative analysis showed a significant increase of acute axonal damage in the medial and lateral parts of the corpus callosum for RFX4_v3 +/- mice compared to RFX4_v3 +/+ mice (medial $1295 \pm 138$ vs. $176 \pm 72$ positive APP spheroids per $\mathrm{mm}^{2},{ }^{* \star *} \mathrm{p}<0.001$; lateral $924 \pm 149$ vs. $343 \pm 51$ positive APP spheroids per $\mathrm{mm}^{2},{ }^{* *} \mathrm{p}<0.01$ ) (Figure 13). 


\subsection{Extensive microglia accumulation in the corpus callosum of RFX4_v3} conditional knockout mice after 4 weeks of cuprizone treatment

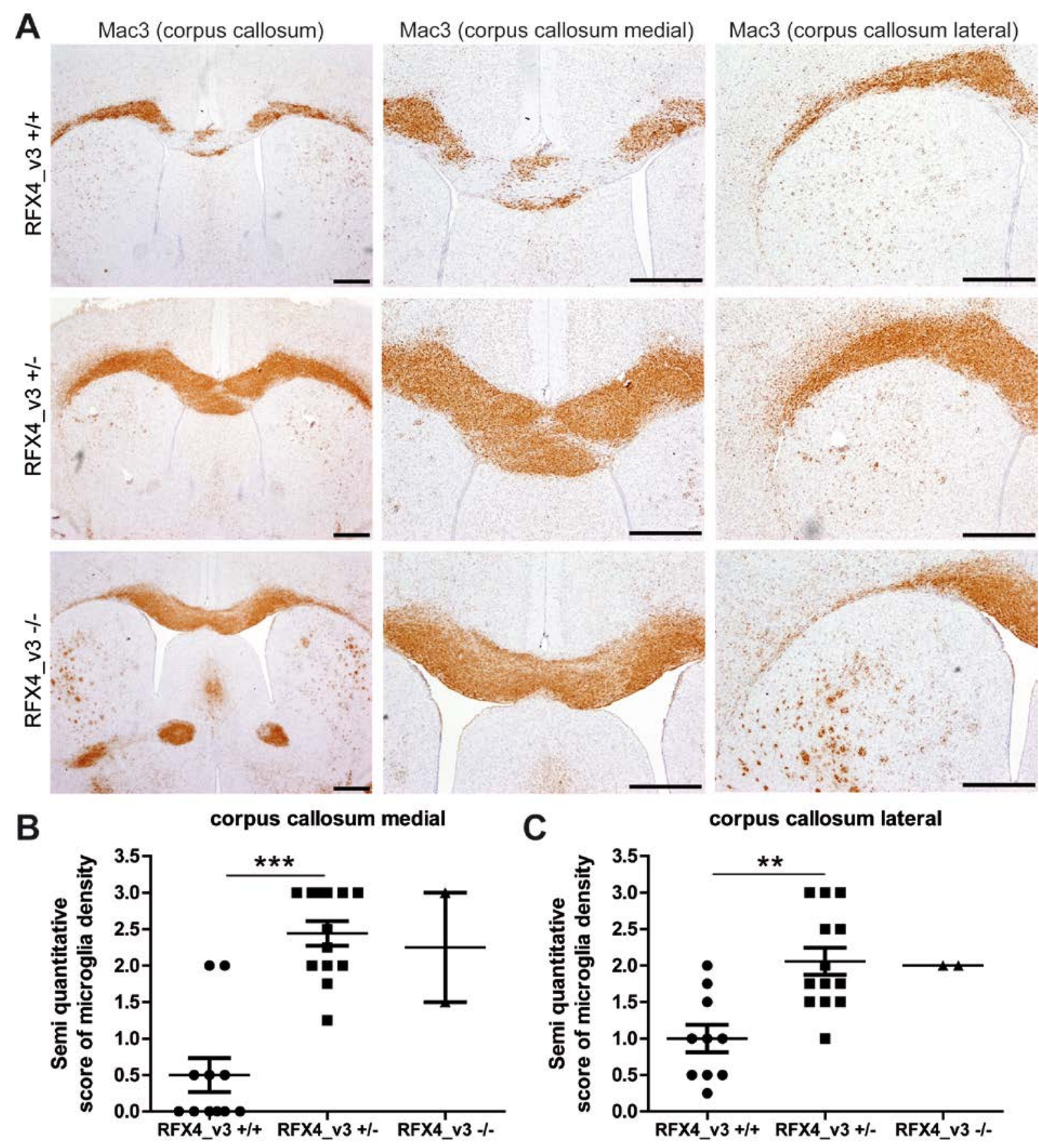

Figure 14: Extensive accumulation of microglia/macrophages in the corpus callosum of RFX4_v3 conditional knockout mice during demyelination.

(A) Mac3 IHC of corpus callosum sections from RFX4_v3 +/+, RFX4_v3 +/- and RFX4_v3 -/- mice after cuprizone treatment. Representative sections are shown. (B-C) Quantification of Mac3 IHC in corpus callosum medial (B) and lateral (C) of indicated mice after 4 weeks of cuprizone intoxication. A score of 0 represents the presence of few microglia/macrophages with low immune reactivity and score of 3 represents high density of microglia/macrophages with intense immune reactivity in the 
corpus callosum. ( ${ }^{* \star} p<0.01,{ }^{* \star *} p<0.001$, Kruskal-Wallis test with Dunn's post test). Data are presented as mean \pm SEM. Scale bar $=500 \mu \mathrm{m}$

Beside enhanced demyelination and axonal damage increased cellularity in the demyelinated corpus callosum was observed in RFX4 deficient mice after week 4 of cuprizone challenge (Figure 11 and Figure 13). Earlier studies demonstrated an increased response of microglia/ macrophages in the demyelinated corpus callosum to clear myelin debris (Hiremath et al., 1998). Therefore, I analyzed microglia/macrophage accumulation by Mac3 staining in the corpus callosum.

Mac3 signals were scored in brain sections of RFX4_v3 +/+, RFX4_v3 +/- and RFX4_V3 -/- mice. Score of 0 represents the presence of few microglia/ macrophages with low immune reactivity and score of 3 represents high density of microglia/ macrophages with intense immune reactivity in the corpus callosum. After week 4 of cuprizone treatment extensive microglia/ macrophage accumulation was observed in RFX4_v3 deficient mice compared to wild type (Figure 14 A). Quantitative analysis revealed that microglia/ macrophages significantly accumulated in larger numbers in the corpus callosum from RFX4 deficient animals compared to wild type animals (score medial $2.4 \pm 0.16$ vs. $0.5 \pm 0.2,{ }^{* \star *} p<0,001$; score lateral $2 \pm 0.18$ vs. $1 \pm 0.18,{ }^{*} p<0.01$ ) (Figure $14 \mathrm{~B} / \mathrm{C}$ ).

\subsection{Blood brain barrier is closed in RFX4_v3 deficient mice}

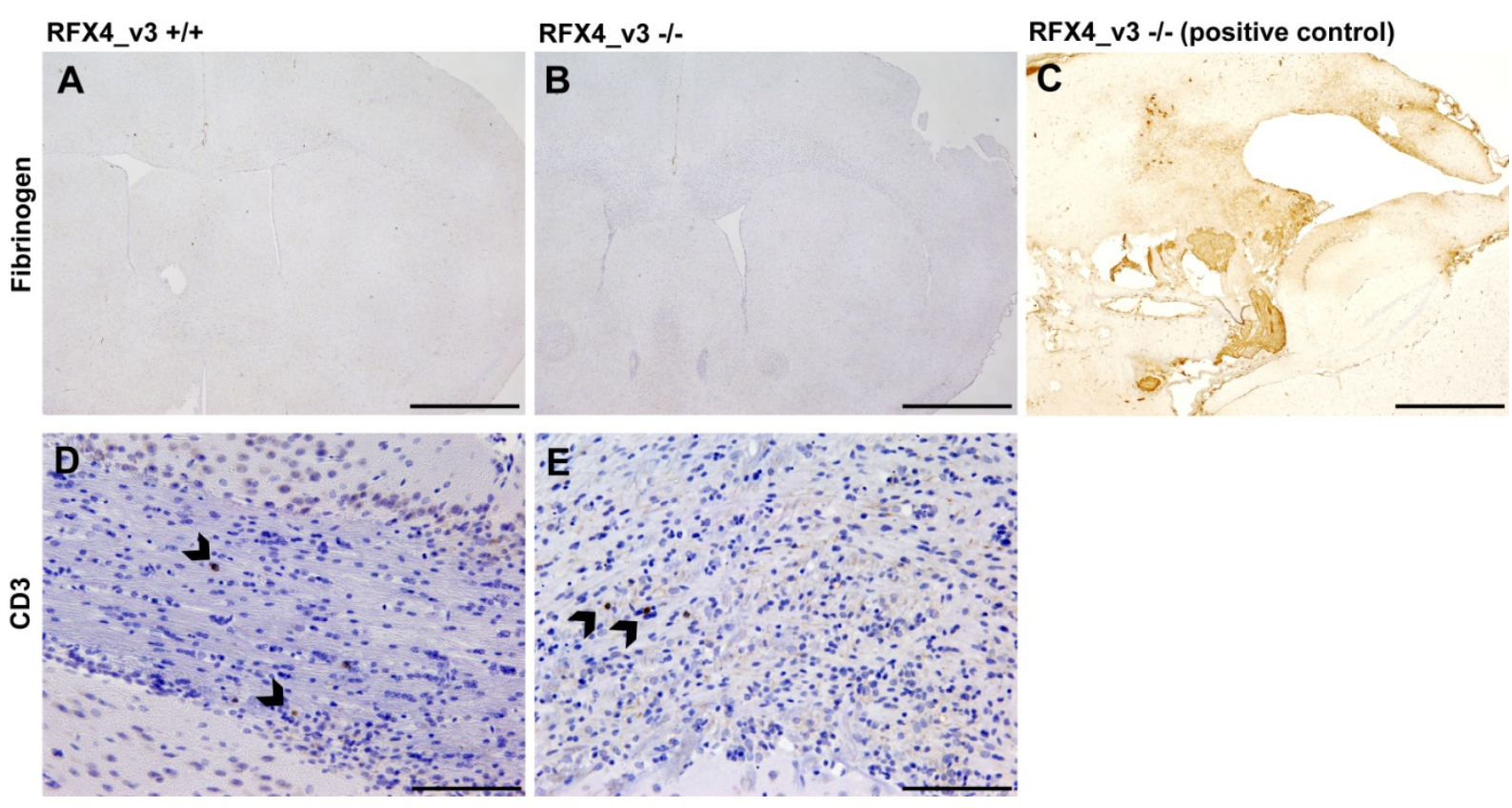

Figure 15: Blood brain barrier is closed in RFX4_v3 deficient mice w/o hydrocephalus.

(A-B) Fibrinogen IHC of brain sections from RFX4_v3 +/+ and RFX4_V3 -/- mice after cuprizone treatment. (C) Fibrinogen staining of RFX4_v3 -/- mice with severe hydrocephalus and brain hemorrhage (positive control). Representative sections are shown. (D-E) CD3 staining of brain 
sections from RFX4_V3 +/+ and RFX4_V3 -/- mice. Representative sections are shown. Scale bar $(A, B, C)=1 \mathrm{~mm} ;(D, E)=100 \mu \mathrm{m}$

Previous studies have demonstrated that the BBB is closed in the cuprizone mouse model, which allows to study the processes of de- and remyelination without substantial influence of the peripheral immune system (Bakker and Ludwin, 1987; Kondo et al., 1987). Because of increased numbers of phagocytes in the corpus callosum of RFX4_v3 deficient mice during cuprizone exposure I investigated whether the BBB remained intact in RFX4_v3 deleted mice.

Therefore, IHC for fibrinogen was performed in cuprizone treated wild type and RFX4_v3 conditional knockout mice (Figure 15). As positive control I used a RFX4_v3 -/mouse with severe hydrocephalus and brain hemorrhage. A positive immunolabeling for fibrinogen was observed in the RFX4_v3 -/- mouse with severe hydrocephalus, but was not detected in RFX4_v3 deficient mice. Thus the BBB of RFX4_v3 deficient mice remain intact under cuprizone challenge, indicating that the Mac3 positive cells most likely represent microglia, but not blood-borne macrophages.

To assess numbers of $T$ lymphocytes in the corpus callosum of wild type and RFX4 deficient mice IHC was performed with an antibody against CD3 (Figure 15). Very few CD3 positive T lymphocytes were detected in both RFX4_v3 +/+ and RFX4_v3 -/- mice. This provides further evidence that the BBB in RFX4_v3 deficient is closed under cuprizone challenge as it is the case for wild type mice. 


\subsection{RFX4_v3 deficiency in astrocytes increases the number of microglia in the} corpora callosa without differential activation patterns
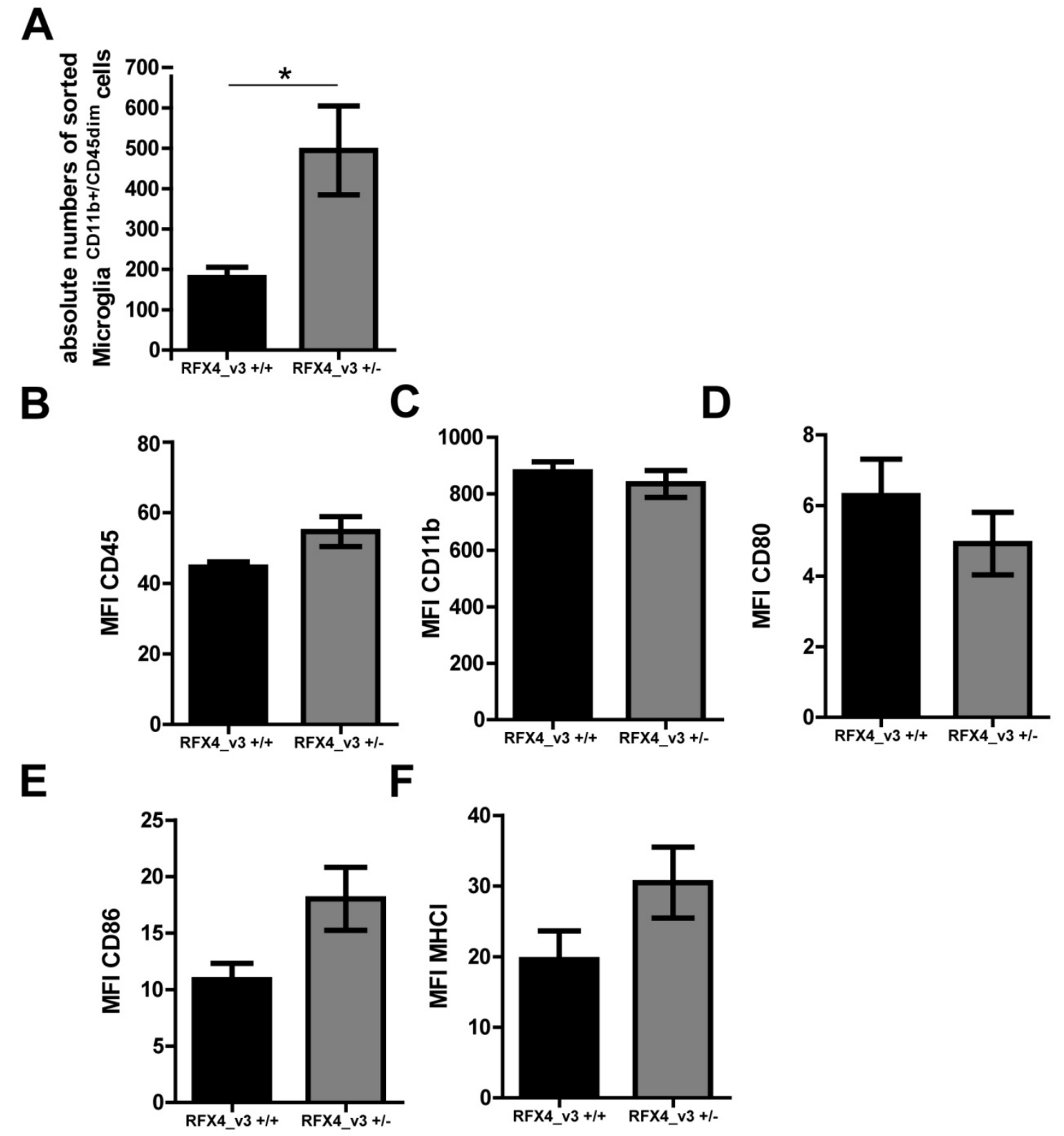

$\mathbf{F}$

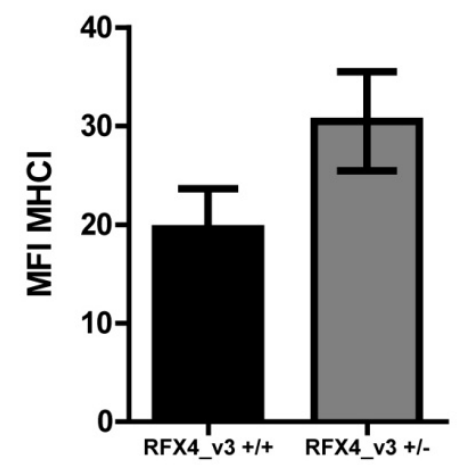

Figure 16: RFX4_v3 deficiency in astrocytes increases the number of microglia in the corpus callosum after cuprizone feeding without induction of microglial activation markers.

(A) Flow cytometry analysis of CD45 and CD11b-positive cells of dissociated corpora callosa of wild type and RFX4_v3 deficient animals after week 4 of cuprizone challenge. RFX4_v3 +/+: $n=7$, RFX4_v3 +/-: $n=6,{ }^{*} p<0,05$, (unpaired t-test). (B-F) Flow cytometry analysis of microglia from corpora callosa for activation markers after cuprizone treatment of RFX4_v3 +/+ or RFX4_v3 +/- mice. Microglia samples were gated on CD11b-positive, CD45-dim cells for microglia. The mean fluorescence intensity was examined for (B) CD11b ( $n=7$ per group, $p>0.05$, unpaired t-test), (C) CD45 ( $n=7$ per group, $p>0.05$, unpaired t-test), (D) CD86 ( $n=7$ per group, $p>0.05$, unpaired t-test) (E) CD80 ( $n=7$ per group, $p>0.05$, unpaired t-test) and $(F)$ MHC class I $(n=7$ per group, $p>0.05$, unpaired t-test). Data are presented as mean \pm SEM. 
The histological characterization of microglia cells in cuprizone-treated mice demonstrated enhanced microglia/ macrophage accumulation in the corpus callosum of RFX4 deficient mice compared to control mice. To further characterize the microglia phenotype of the demyelinated corpus callosum of RFX4 conditional knockout animals and control mice I used flow cytometry analysis.

In a first step dissociated corpora callosa were analyzed for CD45 and CD11b-positive cells by flow cytometry analysis to quantify the number of microglia in wild type and RFX4_v3 deficient animals after week 4 of cuprizone ingestion. Absolute numbers of microglia from RFX4_v3 +/- deficient animals were significantly increased after 4 weeks of cuprizone treatment compared to wild type animals (179 \pm 25 vs. $\left.494 \pm 110,{ }^{*} p<0,05\right)$ (Figure $16 \mathrm{~A}$ ).

In a second step microglia from corpora callosa were analyzed for expression of activation markers using flow cytometry at week 4 after cuprizone treatment (Figure 16 BF). For analysis of expression of activation markers on microglia, samples were gated on CD11b-positive CD45 dim cells for microglia. The mean fluorescence intensity was examined for CD11b, CD45, CD86, CD80 and MHC class I. For none of the analyzed activation markers a significant difference between RFX4_v3 +/- deficient mice and control mice was observed.

Loss of RFX4_v3 in astrocytes thus leads to an increase of microglia in the corpus callosum, but does not significantly alter the expression of activation markers on microglia during demyelination compared to wild type. 


\subsection{Higher numbers of proliferating cells are present in the corpus callosum of RFX4_v3 mutants}

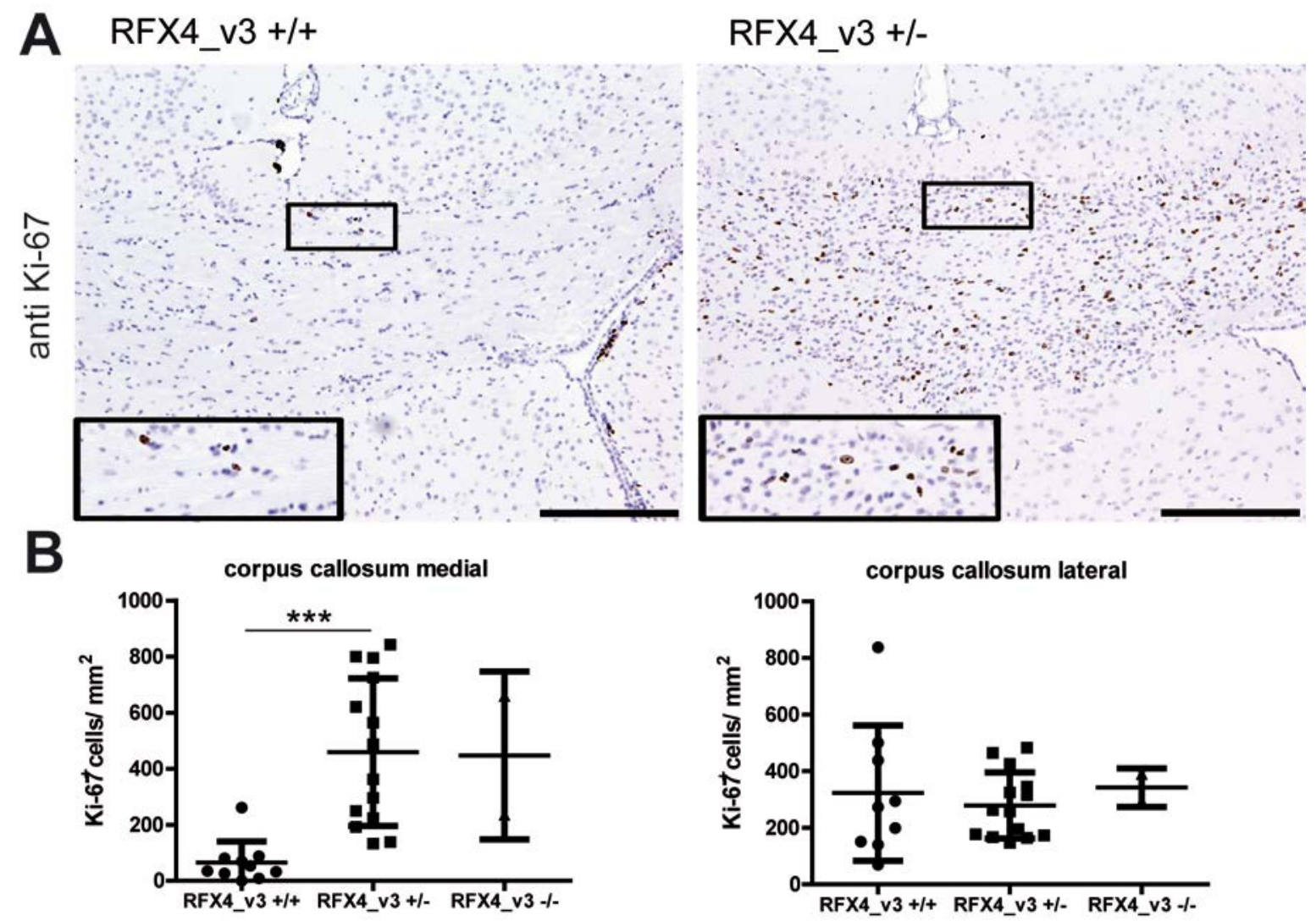

Figure 17: Elevated numbers of proliferating cells in the corpora callosa of RFX4-deficient animals.

(A) IHC for Ki-67 in wild type and RFX4_V3 conditional knockout mice brain sections after cuprizone treatment (B) Quantification of Ki-67 positive cells in the medial and lateral corpus callosum of indicated mice after cuprizone treatment ${ }^{* \star *} \mathrm{p}<0.001 \mathrm{Kruskal}$-Wallis test with Dunn's post test). Data are presented as mean \pm SEM. Scale bar $=200 \mu \mathrm{m}$.

To explain the increased microglia numbers in the demyelinated area I considered whether higher numbers of proliferating cells are detectable in the corpus callosum of RFX4_V3 deficient mice. Therefore IHC for Ki-67 was performed from wild type and RFX4_v3 conditional knockout mice to assess numbers of proliferating cells (Figure 17 A).

Significantly higher numbers of Ki-67 positive cells could be detected in the medial part of RFX4_v3 +/- animals compared to control animals (score medial $459 \pm 70$ vs. $65 \pm$ $23 \mathrm{Ki}-67$ positive cells per $\mathrm{mm}^{2},{ }^{* *} \mathrm{p}<0,001$ ) (Figure $17 \mathrm{~B}$ ). 


\subsection{Reduced numbers of oligodendrocytes in the corpus callosum of RFX4_v3} deficient mice after cuprizone exposure
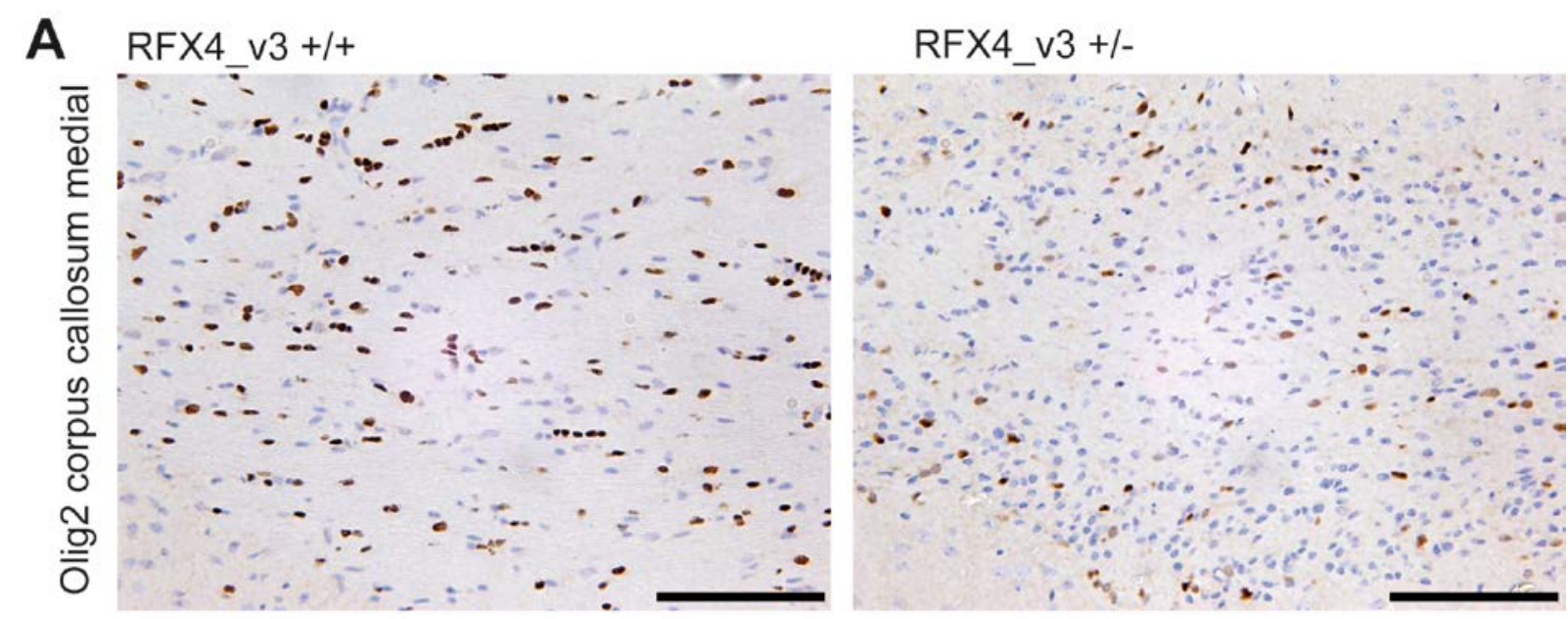

B

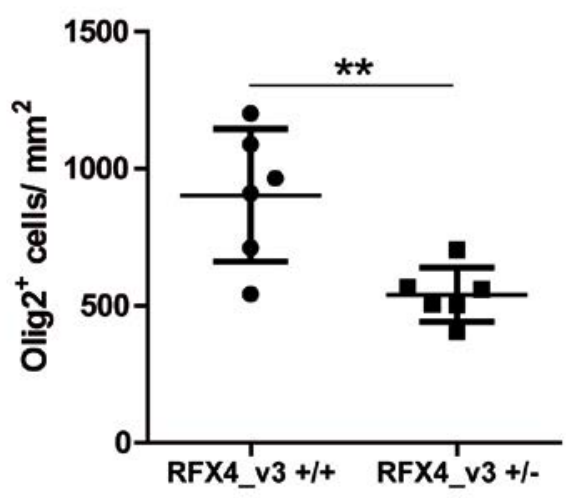

Figure 18: Reduced numbers of oligodendrocytes in the corpus callosum of RFX4_v3 deficient mice after cuprizone exposure.

(A) Immunostaining using the oligodendrocyte marker anti-Olig2 of brain sections from RFX4 deficient animals and wild type animals. Representative images are shown. (B) Quantification of Olig2 staining in corpus callosum medial of indicated mice $\left({ }^{* *} p<0.01\right.$, unpaired $t$ test). Data are presented as mean \pm SEM. Scale bar $=100 \mu \mathrm{m}$

To assess the total number of oligodendrocytes after week 4 of cuprizone treatment I stained sections from RFX4_v3 +/+ and RFX4_v3 +/- mice with an antibody against Olig2, a marker for oligodendrocyte precursor cells (OPCs) and mature oligodendrocytes (Figure 18). RFX4_v3 +/- mice showed significantly lower numbers of oligodendrocytes in the medial part of the corpus callosum after week 4 of cuprizone treatment (medial score RFX4_v3 +/- $540 \pm 40$ vs. RFX4_v3 +/+ $903 \pm 98$ Olig2 positive cells per $\left.\mathrm{mm}^{2},{ }^{*} \mathrm{p}<0.05\right)$. 


\subsubsection{RFX4_v3 conditional knockout mice show delayed remyelination}

In the cuprizone mouse model the removal of cuprizone from the diet permits rapid remyelination which is almost complete after 4 weeks.

To analyze their potential for remyelination 7 to 8 week old wild type and GFAP cell specific conditional RFX4_v3 knockout mice were treated for 4 weeks with a $0.25 \%$ cuprizone diet to induce demyelination. For the induction of remyelination, cuprizone infused chow was removed and animals were fed with a normal diet for either 7 days or 14 days. 
3.2.2.2.1 RFX4_v3 conditional knockout mice remain demyelinated after induction of remyelination for 1 week

A Cuprizone Normaldiet week 0
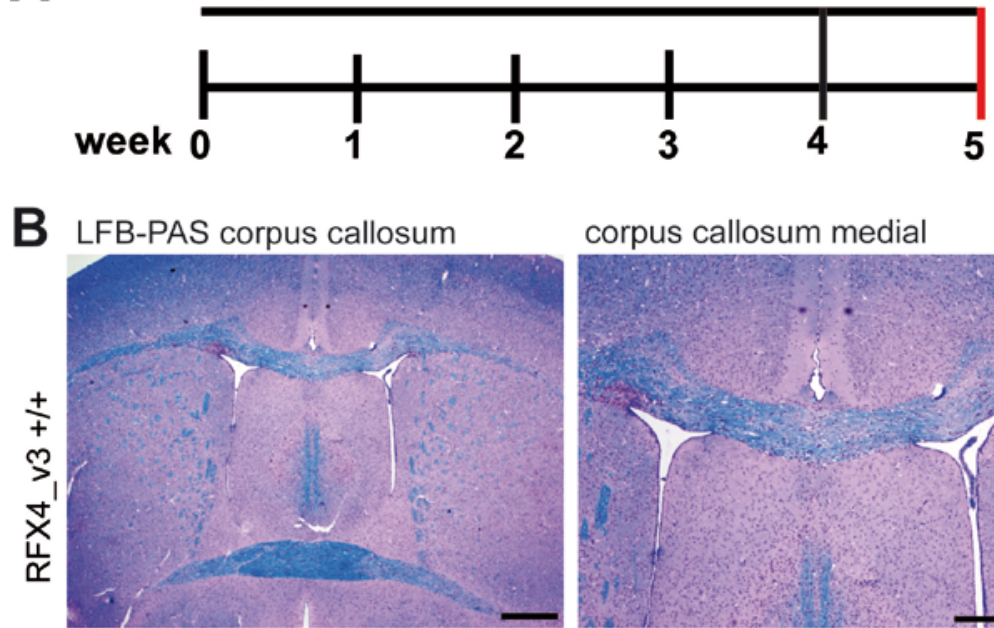

corpus callosum medial corpus callosum lateral
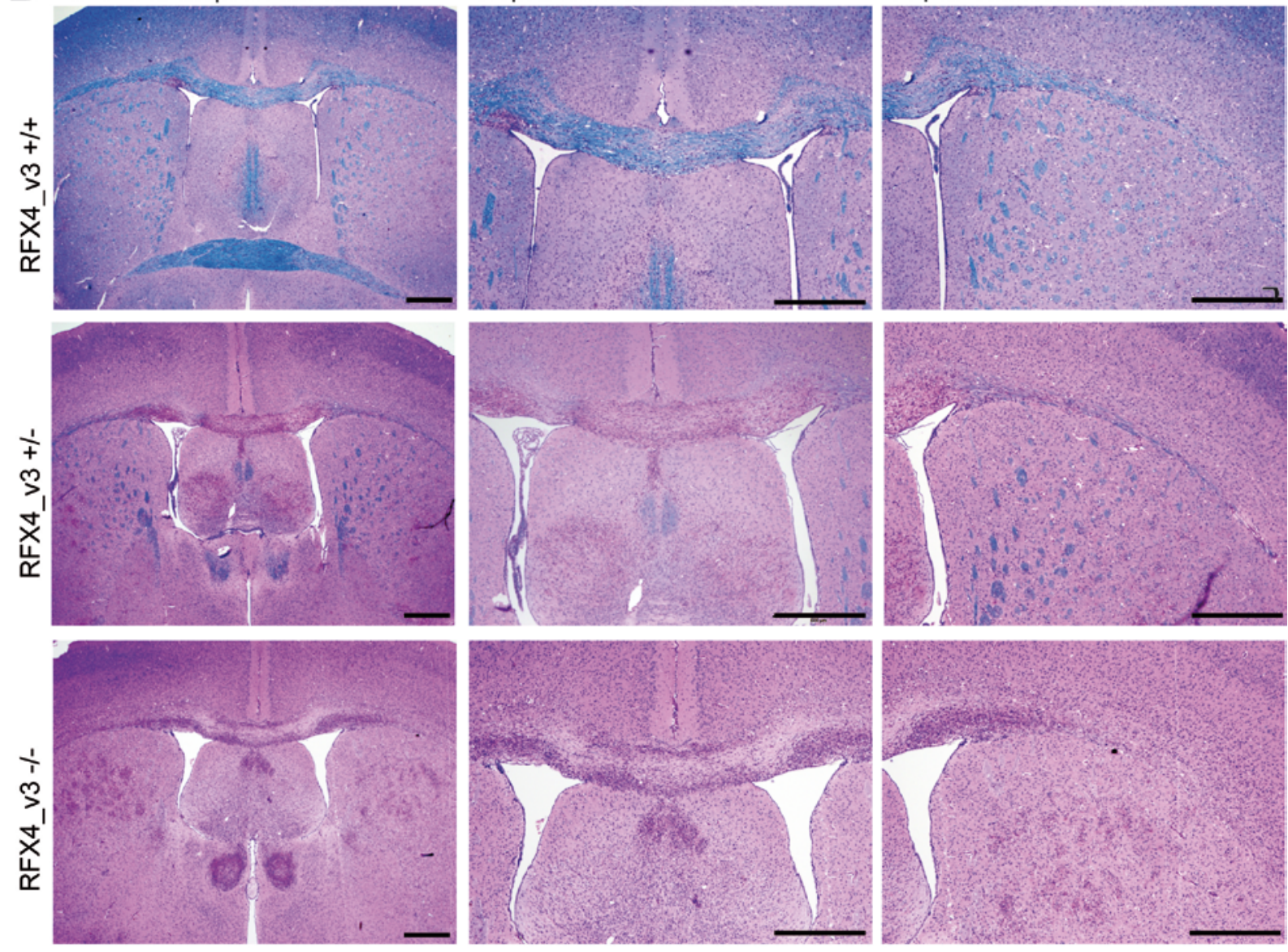

C
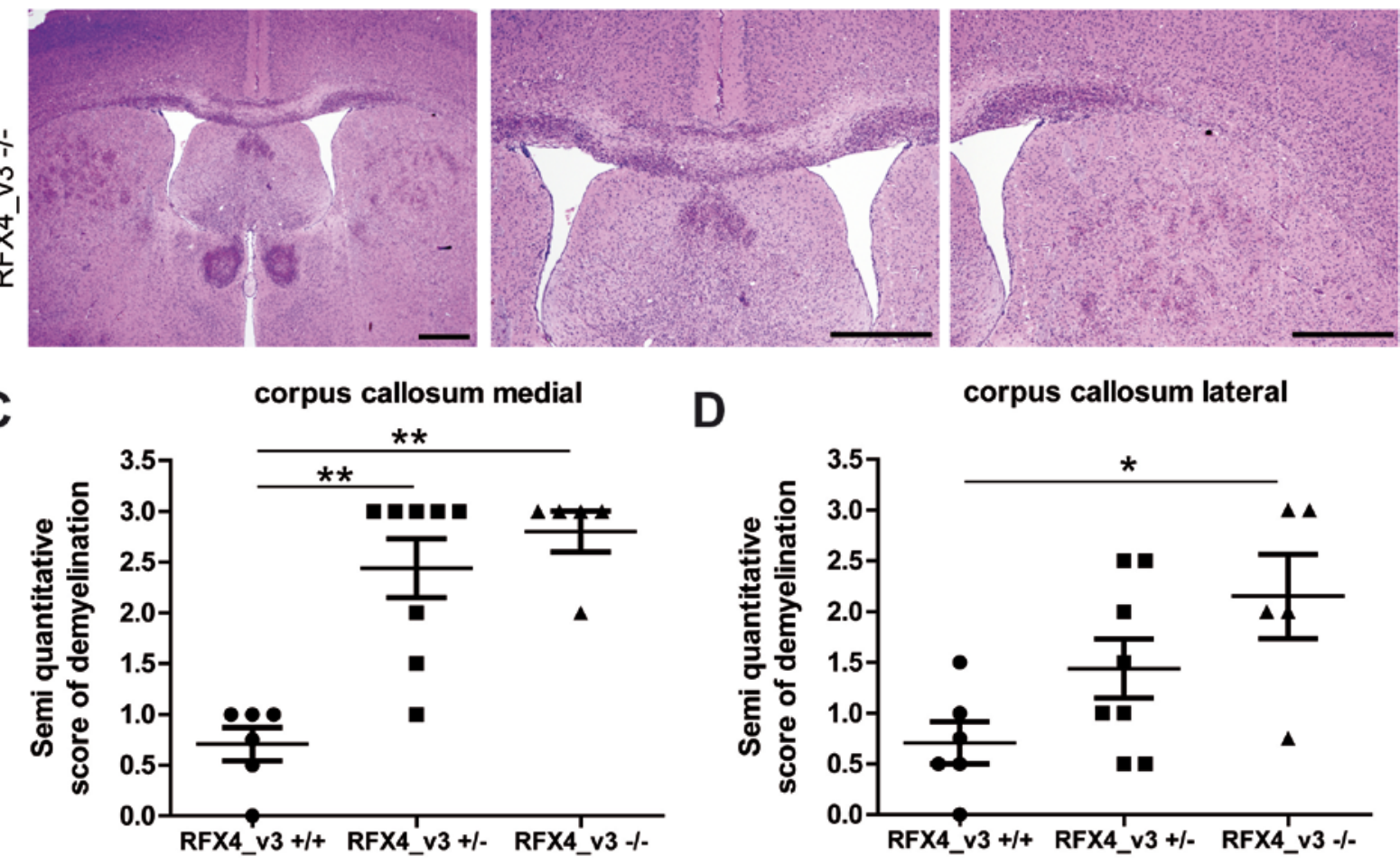

D corpus callosum lateral

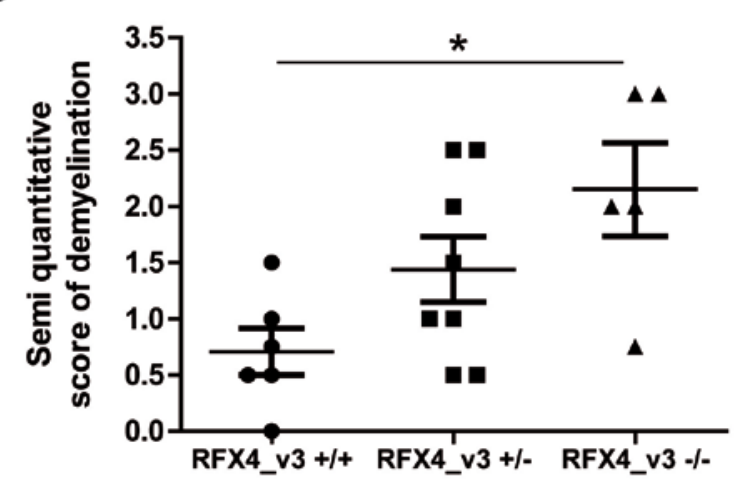

Figure 19: RFX4_v3 conditional knockout mice remain demyelinated after induction of remyelination for 1 week. 
(A) Schematic representation of experimental layout. RFX4_v3 +/+, RFX4_v3 +/- and RFX4_v3 -/mice were treated for 4 weeks with a $0.25 \%$ cuprizone diet followed by one week of a cuprizone-free diet. (B) LFB-PAS staining of representative sections from RFX4_V3 +/+, RFX4_V3 +/- and RFX4_v3 -/- mice. (C-D) Quantification of demyelination by scoring LFB-PAS staining in (C) corpus callosum medial and (D) corpus callosum lateral. Score of 0 represents complete remyelination and score of 3 represents complete demyelination ( ${ }^{*} p<0.05,{ }^{*} p<0.01$, one way ANOVA with Bonferroni post test; Kruskal-Wallis test with Dunn's post test). Data are presented as mean \pm SEM. Scale bar $=500 \mu \mathrm{m}$

A

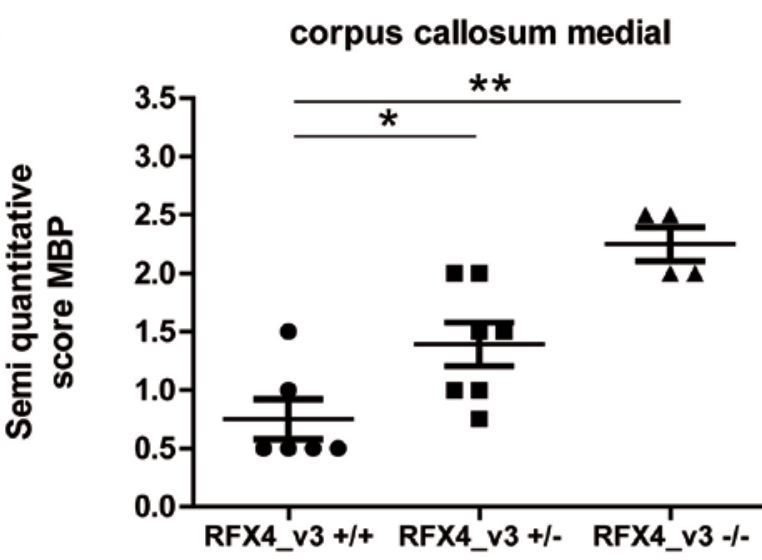

C

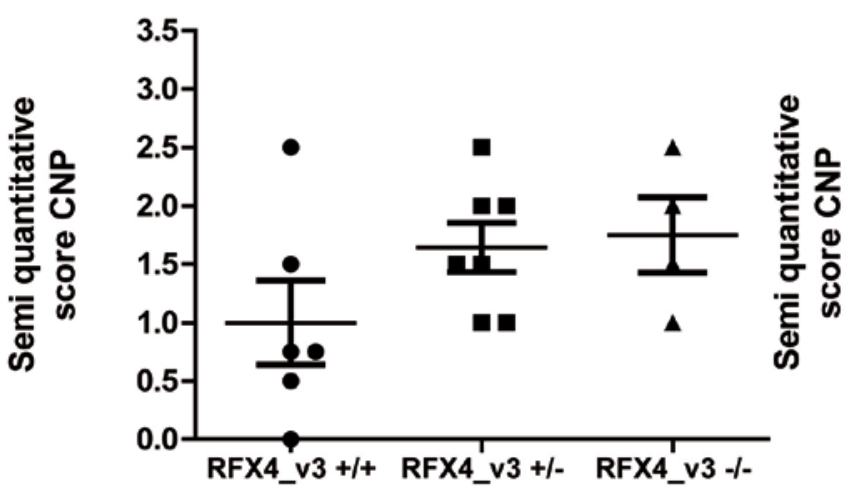

B corpus callosum lateral
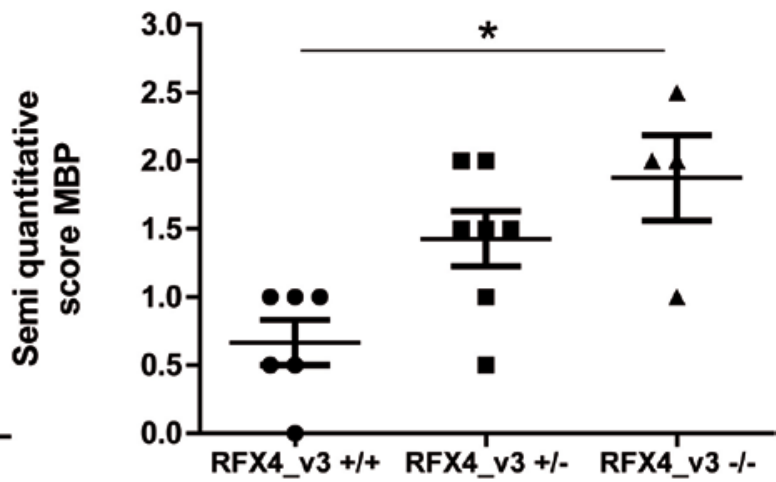

D corpus callosum lateral

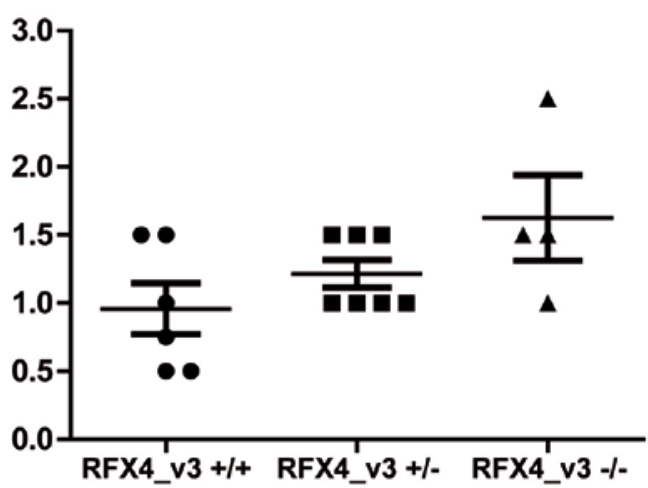

Figure 20: RFX4_v3 conditional knockout mice remain demyelinated after induction of remyelination for 1 week.

Brain sections from RFX4_V3 +/+, RFX4_v3 +/- and RFX4_v3 -/- mice were stained for myelin proteins MBP and CNPase. Extent of remyelination was quantified scoring (A-B) MBP and (C-D) CNPase signal intensity in corpus callosum medial $(\mathbf{A}, \mathbf{C})$ and corpus callosum lateral $(\mathbf{B}, \mathbf{D})\left({ }^{*} p<0.05\right.$, ** $p<0.01$, Kruskal-Wallis test with Dunn's post test). Score of 0 represents complete remyelination and score of 3 represents complete demyelination. Data are presented as mean \pm SEM.

Remyelination was first analyzed 1 week after cuprizone withdrawal. Sections from RFX4_v3 +/+, RFX4_v3 +/- and RFX4_v3 -/- mice were examined and scored for remyelination by LFB-PAS staining (Figure 19). A score of 0 represents complete remyelination and score of 3 represents complete demyelination. RFX4_v3 deficient animals remained demyelinated after 1 week cuprizone withdrawal. The corpus callosum 
of RFX4_v3 deficient mice was still demyelinated, whereas the myelin of control mice appeared to be remyelinated after 1 week cuprizone cessation (Figure 19 B). Quantitative analysis of the medial and lateral part of the corpus callosum revealed a significantly lower level of remyelination in RFX4_v3 conditional knockout animals compared to control mice (medial score RFX4_v3 -/-: $2.8 \pm 0.2$; RFX4_v3 +/-: $2.4 \pm 0.3$ vs. RFX4_v3 +/+: $0.7 \pm 0.2 ;{ }^{*} p<0.05 * * p<0.01$ ) (lateral score RFX4_v3 -/-: $2.1 \pm 0.4 ;$ RFX4_v3 +/-: $1.4 \pm$ 0.3 vs. RFX4_v3 +/+: $0.7 \pm 0.2 ;{ }^{*} p<0.05 * * p<0.01$ ) (Figure $19 \mathrm{C} / \mathrm{D}$ ).

These results were confirmed by analyzing the content of myelin proteins in the corpus callosum of control mice and RFX4_v3 deficient animals. The extent of remyelination was evaluated by scoring sections from RFX4_v3 +/+, RFX4_v3 +/- and RFX4_v3 -/- mice immunostained for myelin proteins MBP and CNPase. Myelin protein MBP was still significantly reduced in RFX4 deficient animals compared to wild type animals (medial score RFX4_v3 -/- $1.9 \pm 0.3$; RFX4_v3 +/- $1.4 \pm 0.2$ vs. RFX4_v3 +/+ $0.75 \pm 0.2,{ }^{*} p<0.05$, ${ }^{* *} p<0.01$ ) (lateral score RFX4_v3 -/- $2 \pm 0.3$ vs. RFX4_v3 $+/+0.7 \pm 0.2,{ }^{*} p<0.05$ ) (Figure 20 A, B). However no significant difference between wild type and RFX4_v3 conditional knockout animals was observed for the myelin protein CNPase ( $p>0.05)$ (Figure $20 \mathrm{C}, \mathrm{D})$, indicating that the process of remyelination was active also in RFX4_v3 deficient mice. 


\subsection{Microglia accumulation in the corpus callosum of RFX4_v3 conditional} knockout animals is still increased after 1 week cuprizone withdrawal

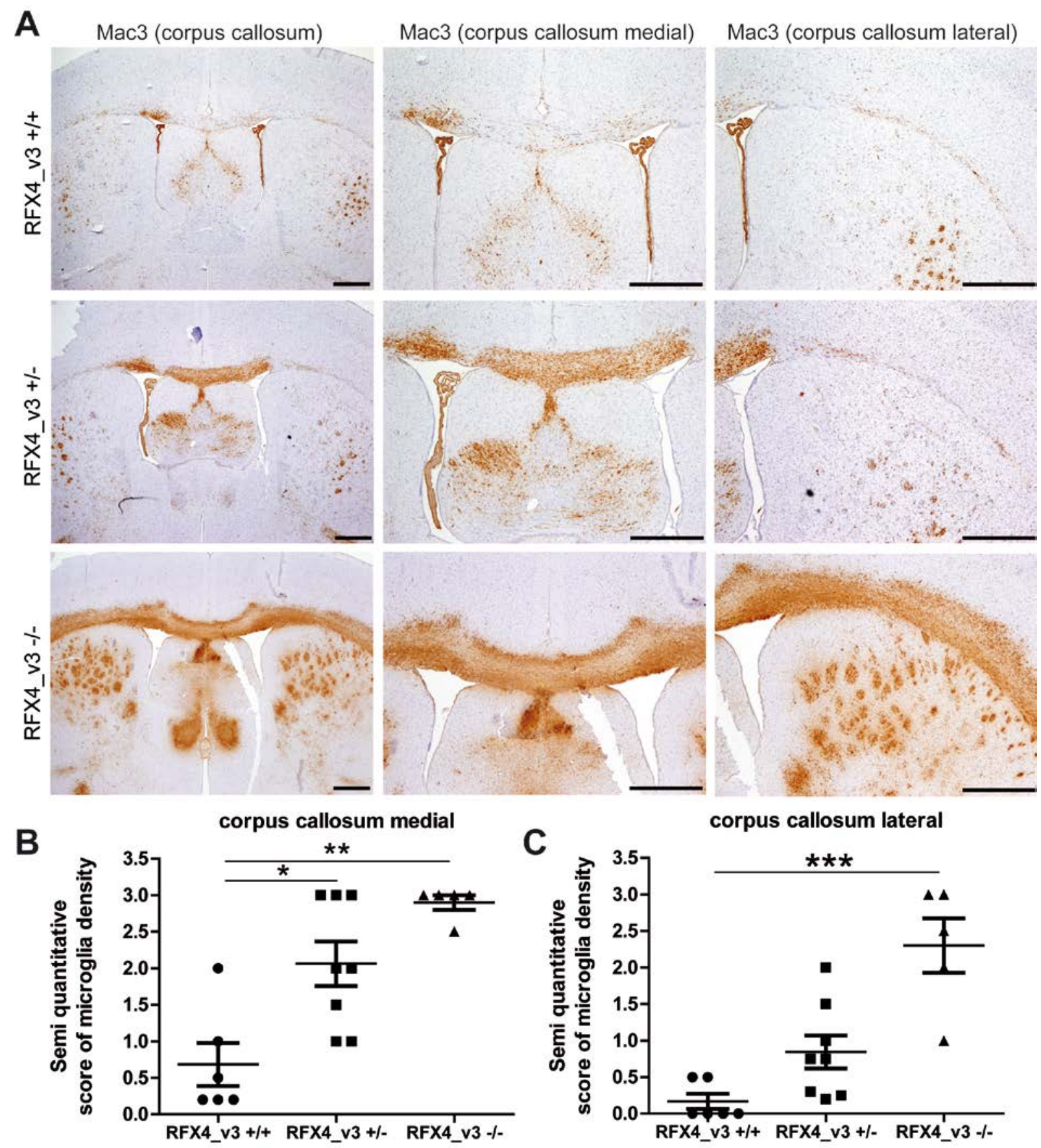

Figure 21: Microglia accumulation in RFX4_v3 conditional knockout animals is still increased after 7 days cuprizone withdrawal.

(A) Mac3 IHC of brain sections from RFX4_V3 +/+, RFX4_V3 +/- and RFX4_V3 -/- mice 1 week after cuprizone cessation. Representative sections are shown. (B-C) Quantification of Mac3 IHC in medial (B) and lateral (C) corpora callosa of the different genotypes after 4 weeks of cuprizone intoxification. A score of 0 represents the presence of few microglia/macrophages with low immune reactivity and score of 3 represents high density of microglia/macrophages with high immune reactivity in the corpus 
callosum ( ${ }^{\star} p<0,05,{ }^{* *} p<0,01,{ }^{* * *} p<0,001$, Kruskal-Wallis test with Dunn's post test). Data are presented as mean \pm SEM. Scale bar $=500 \mu \mathrm{m}$

With the onset of remyelination after removal of cuprizone from the diet Mac3-positive microglia cells usually begin to decrease in the corpus callosum.

I thus analyzed microglia/ macrophage accumulation during remyelination by antiMac3 IHC of sections from RFX4_v3 +/+, RFX4_v3 +/- and RFX4_v3 -/- mice after one week of cuprizone cessation. As predicted, cuprizone removal led to a reduction of microglia/macrophage accumulation in wild type animals whereas RFX4_v3 deficient animals retained a high infiltration rate of microglia/macrophage cells. Quantitative analysis revealed a significant difference in the medial part of the corpus callosum for both RFX4_v3 +/- and RFX4_v3 -/- versus control mice (medial score RFX4_v3 -/-: $2.9 \pm$ 0.1; RFX4_v3 +/-: $2.1 \pm 0.3$ vs. RFX4_v3 +/+: $\left.0.7 \pm 0.3,{ }^{* *} p<0.01,{ }^{*} p<0.05\right)$ (Figure $\left.21 \mathrm{~B}\right)$. In the lateral part of the corpus callosum a significant difference was only observed between RFX4_v3 -/- mice and wild type mice (lateral score RFX4_v3 -/-: $2.3 \pm 0.4$ vs. RFX4_v3 +/+: $0.2 \pm 0.1,{ }^{\star * *} p<0.001$ ) (Figure $21 \mathrm{C}$ ). 


\subsection{Loss of RFX4_v3 in astrocytes restricts oligodendrocyte differentiation} without changing numbers of oligodendrocyte precursor cells

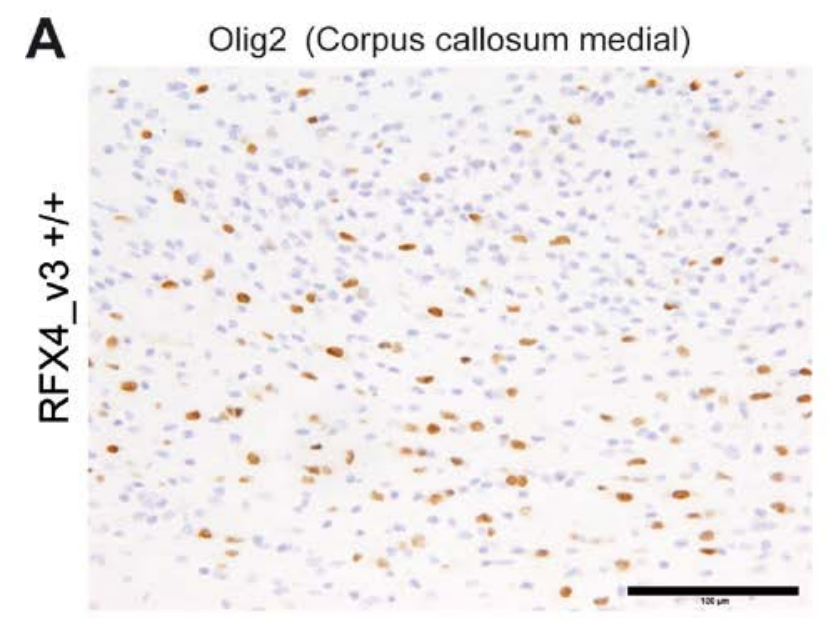

B p25 (Corpus callosum medial)

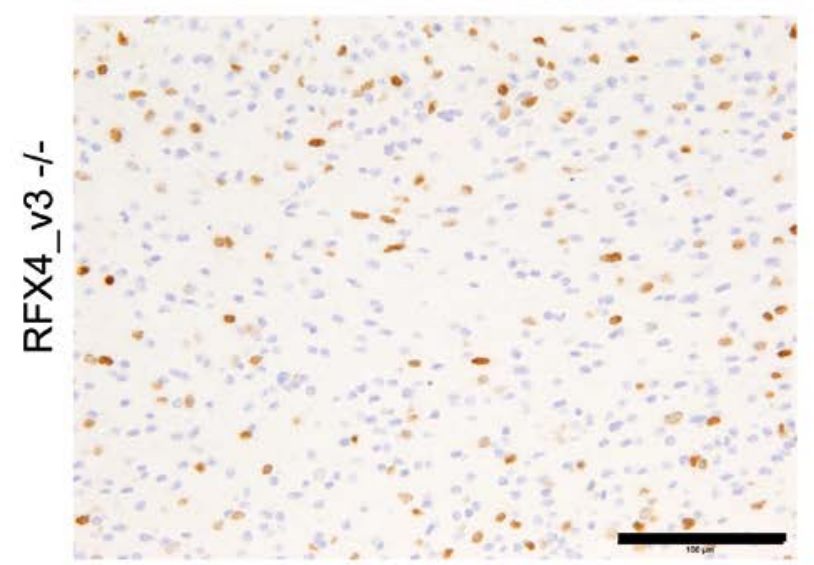

C

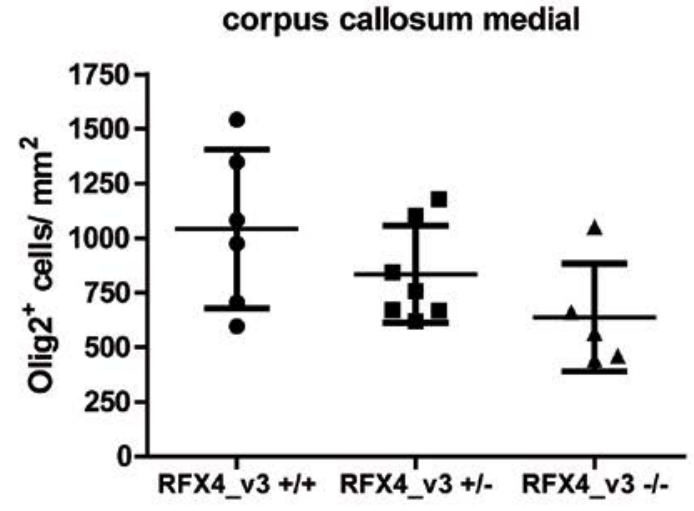

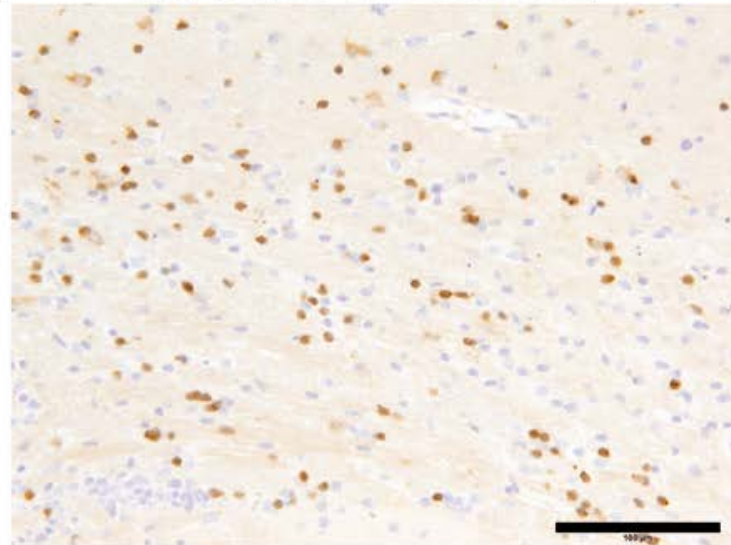

D
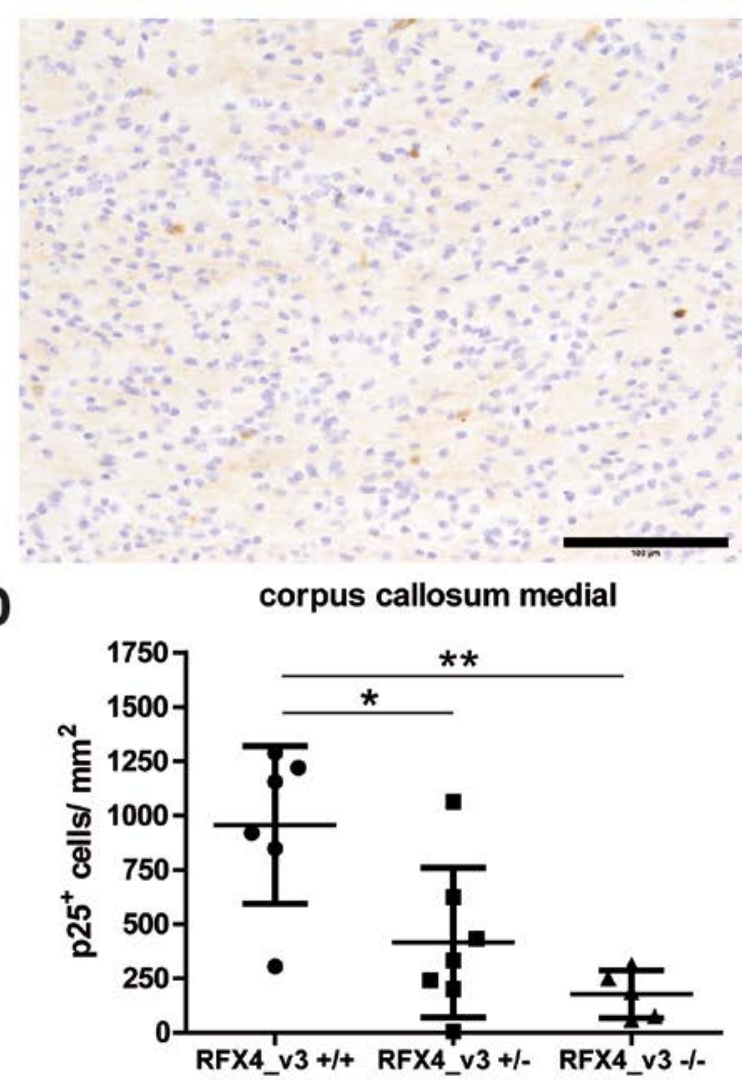

Figure 22: Loss of RFX4_v3 in astrocytes restricts oligodendrocyte differentiation without changing numbers of oligodendrocyte precursor cells.

(A) Immunostaining using the oligodendrocyte marker anti-Olig2 of brain sections from RFX4 deficient animals and wild type animals. Representative images are shown. (C) Quantification of Olig2 IHC in corpus callosum medial. (B) Immunostaining using the mature oligodendrocyte marker anti-p25 of brain sections from RFX4 deficient animals and wild type animals. Representative images are shown. (D) Quantification of p25 IHC in corpus callosum medial of indicated mice $\left({ }^{*} p<0.05,{ }^{*} p<0.01\right.$, one way ANOVA with Bonferroni post test). Data are presented as mean \pm SEM. Scale bar $=100 \mu \mathrm{m}$ 
The process of remyelination depends on the presence of OPCs which differentiate to mature oligodendrocytes to produce the myelin sheath around the axon.

I analyzed oligodendrocyte differentiation during remyelination, by immunostaining for Olig2, a marker for oligodendrocytes, and for p25, a marker for mature oligodendrocytes. Although a slight decrease in numbers of oligodendrocytes in the medial part was observed, loss of astrocytic RFX4_v3 had no significant effect on numbers of Olig2 positive oligodendrocytes (Figure $22 \mathrm{~A}, \mathrm{C}$ ).

However, mature oligodendrocytes were significantly reduced in the medial part of the corpus callosum during the process of remyelination as revealed by p25 IHC (medial score RFX4_v3 -/- 176 +/- 49; RFX4_v3 +/- $414 \pm 131$ vs. RFX4_v3 +/+ $956 \pm 148$ p25 positive cells per $\mathrm{mm}^{2},{ }^{*} \mathrm{p}<0.01,{ }^{*} \mathrm{p}<0.05$ ) (Figure $22 \mathrm{~B}, \mathrm{D}$ ).

Thus, the majority Olig2 positive cells in RFX4_v3 deficient mice represented most likely OPCs and not mature oligodendrocytes. This indicates that RFX4_v3 deletion does not change proliferation of OPCs, but impedes differentiation of OPCs into mature oligodendrocytes. 
3.2.2.2.4 RFX4_v3 conditional knockout mice show delayed remyelination of the corpus callosum compared to wild type mice

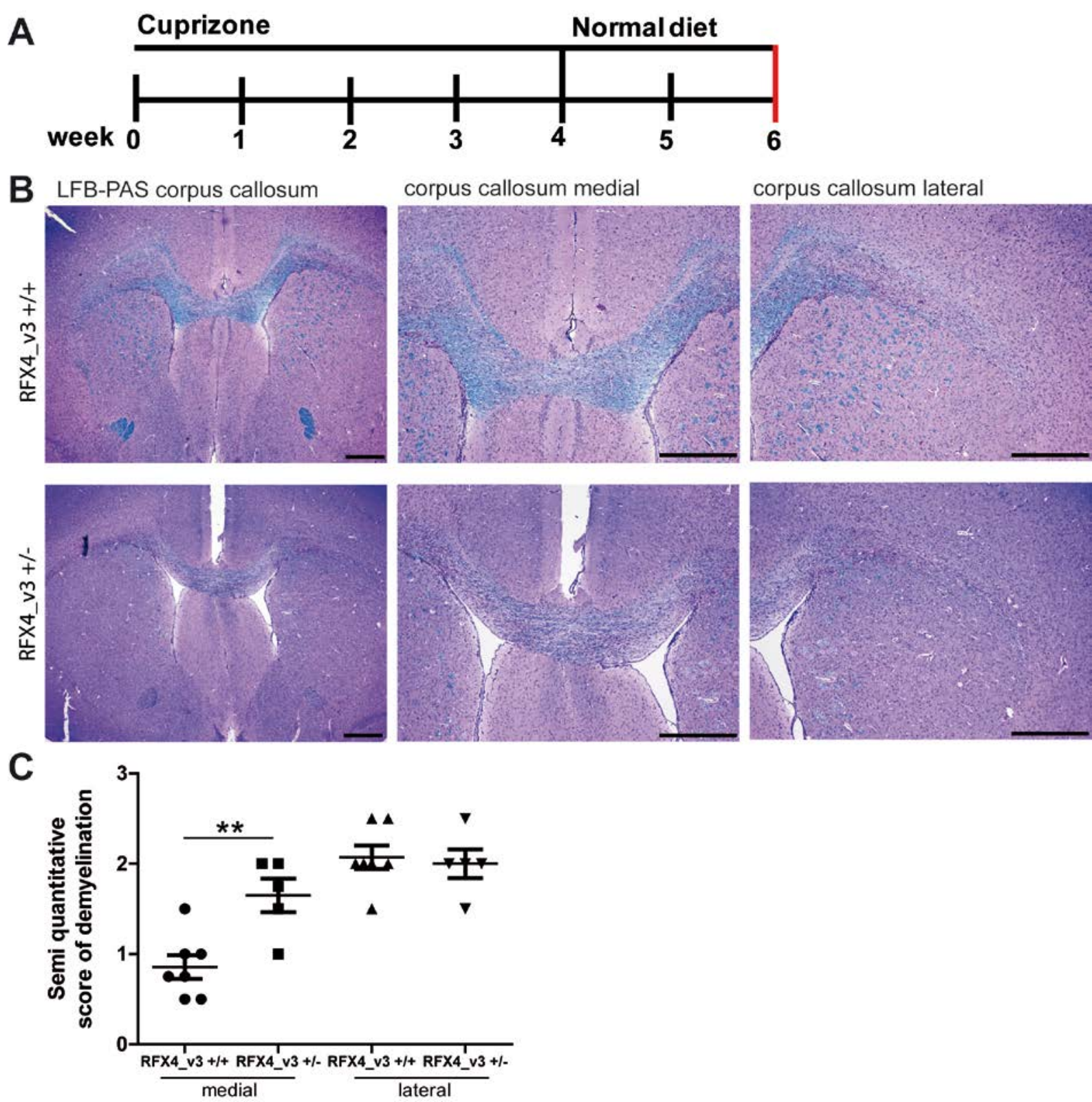

Figure 23: RFX4_v3 conditional knockout mice remyelinate in the corpus callosum with a delay compared to wild type mice.

(A) Schematic representation of the experimental design. RFX4_v3 +/+ and RFX4_v3 +/- were treated for 4 weeks with a $0.25 \%$ cuprizone diet followed by 2 weeks of a cuprizone-free diet. (B) LFB-PAS staining of representative sections from RFX4_v3 +/+ and RFX4_v3 +/- mice. (C) Quantification of demyelination by scoring LFB-PAS staining in corpus callosum medial and lateral. Score of 0 represents complete remyelination and score of 3 represents complete demyelination ${ }^{* \star} p<0.01$, one way ANOVA with Bonferroni post test). Data are presented as mean \pm SEM. Scale bar $=500 \mu \mathrm{m}$

To resolve whether RFX4_v3 deficient mice remain demyelinated in the corpus callosum, remyelination was analyzed after 2 weeks of cuprizone withdrawal. Sections from 
RFX4_v3 +/+, RFX4_v3 +/- were examined and scored for remyelination by LFB-PAS staining. LFB-PAS staining revealed intact myelin in RFX4_v3 deficient animals after 14 days of cuprizone withdrawal. Quantitative analysis of the medial and lateral part of the corpus callosum revealed a significantly lower level of remyelination in RFX4_v3 conditional knockout animals in the medial part compared to control mice (medial score RFX4_v3 +/-: $1.7 \pm 0.2$ vs. RFX4_v3 +/+: $0.9 \pm 0.13$; ${ }^{*} p<0.01$ ) (Figure $23 \mathrm{C}$ ). In the lateral part of corpus callosum no difference was observed

\subsection{Numbers of Mac3 positive stained microglia are comparable in the corpus callosum of RFX4_v3 mutants and wild type animals 2 weeks after cuprizone cessation}

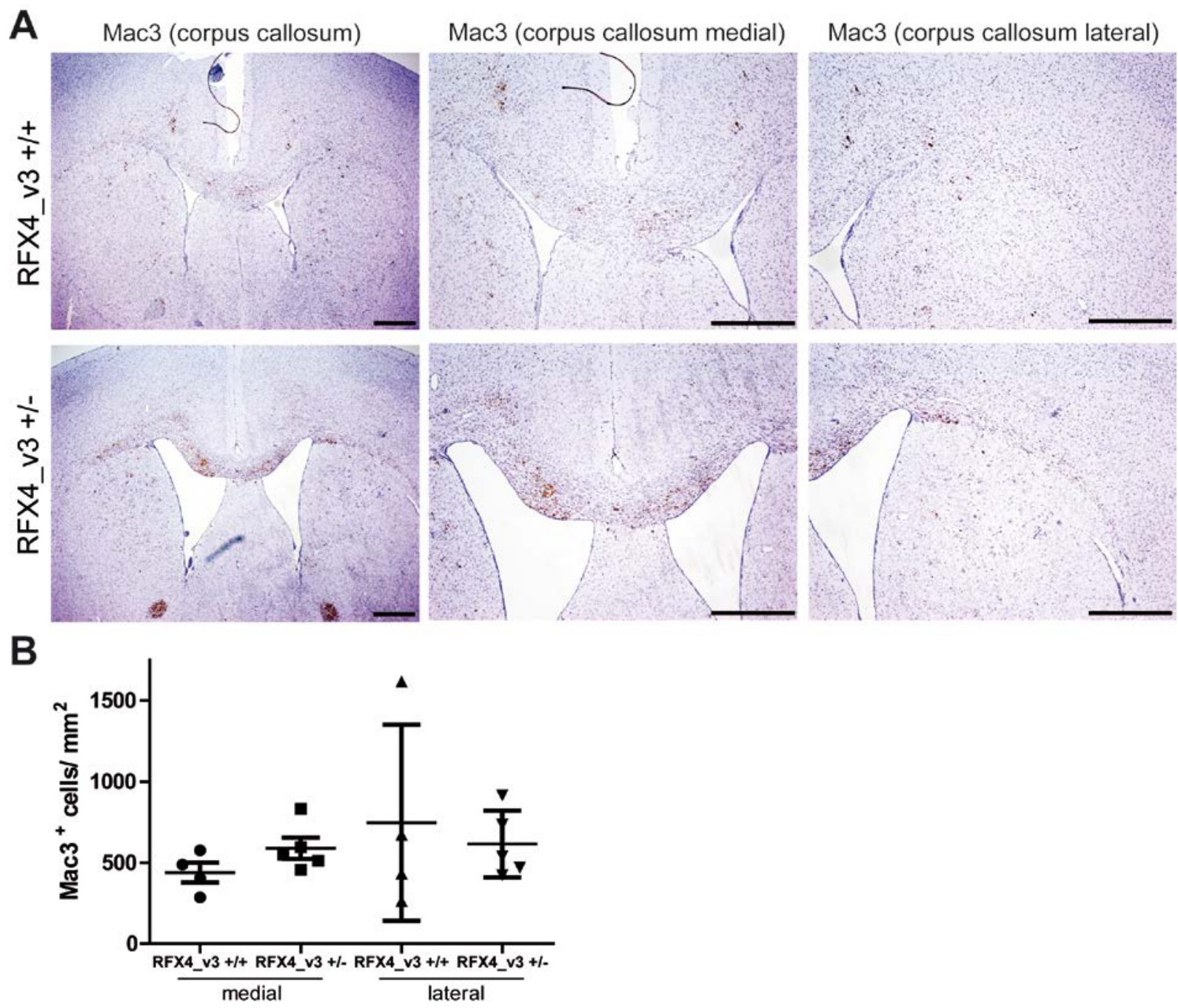

Figure 24: Numbers of Mac3 positive microglia are comparable in the corpus callosum of RFX4_v3 mutants and wild type animals 2 weeks after of cuprizone cessation.

(A) Mac3 IHC of brain sections from RFX4_V3 +/+ and RFX4_v3 +/- mice 2 weeks after cuprizone cessation. Representative sections are shown. (B) Quantification of Mac3 IHC in the corpus callosum medial and lateral of indicated mice 2 weeks after cuprizone withdrawal ( $p>0.05$, Kruskal-Wallis test with Dunn's post test) (B). Data are presented as mean \pm SEM. Scale bar $=500 \mu \mathrm{m}$ 
Microglia accumulation after 2 weeks of remyelination was analyzed by Mac3 IHC of brain sections of RFX4_v3 +/+, RFX4_v3 +/- mice. 2 weeks of cuprizone cessation reduced the amount of microglia accumulation in wild type animals and RFX4_v3 deficient mice (Figure $24 \mathrm{~A}$ ). Quantitative analysis revealed no significant difference between RFX4_v3 deficient mice and wild type mice (Figure $24 \mathrm{~B}$ ).

\subsubsection{Effects of astrocytic RFX4_v3 inhibition are present after 2 weeks of cuprizone challenge}

In order to better understand the cuprizone induced pathology in RFX4_v3 deficient mice I performed a time course experiment to study the course of demyelination based on LFB-PAS histochemistry, of astrogliosis based on GFAP IHC and of microglia activation based on Mac3 IHC. Therefore 7 to 8 week old GFAP cell-specific RFX4_v3 conditional knockout mice without hydrocephalus and control mice (C57BI/6) were treated with a $0.25 \%$ cuprizone diet for $1,2,3$, and 4 weeks. 


\subsection{RFX4_v3 deficiency markedly accelerates demyelination in vivo}

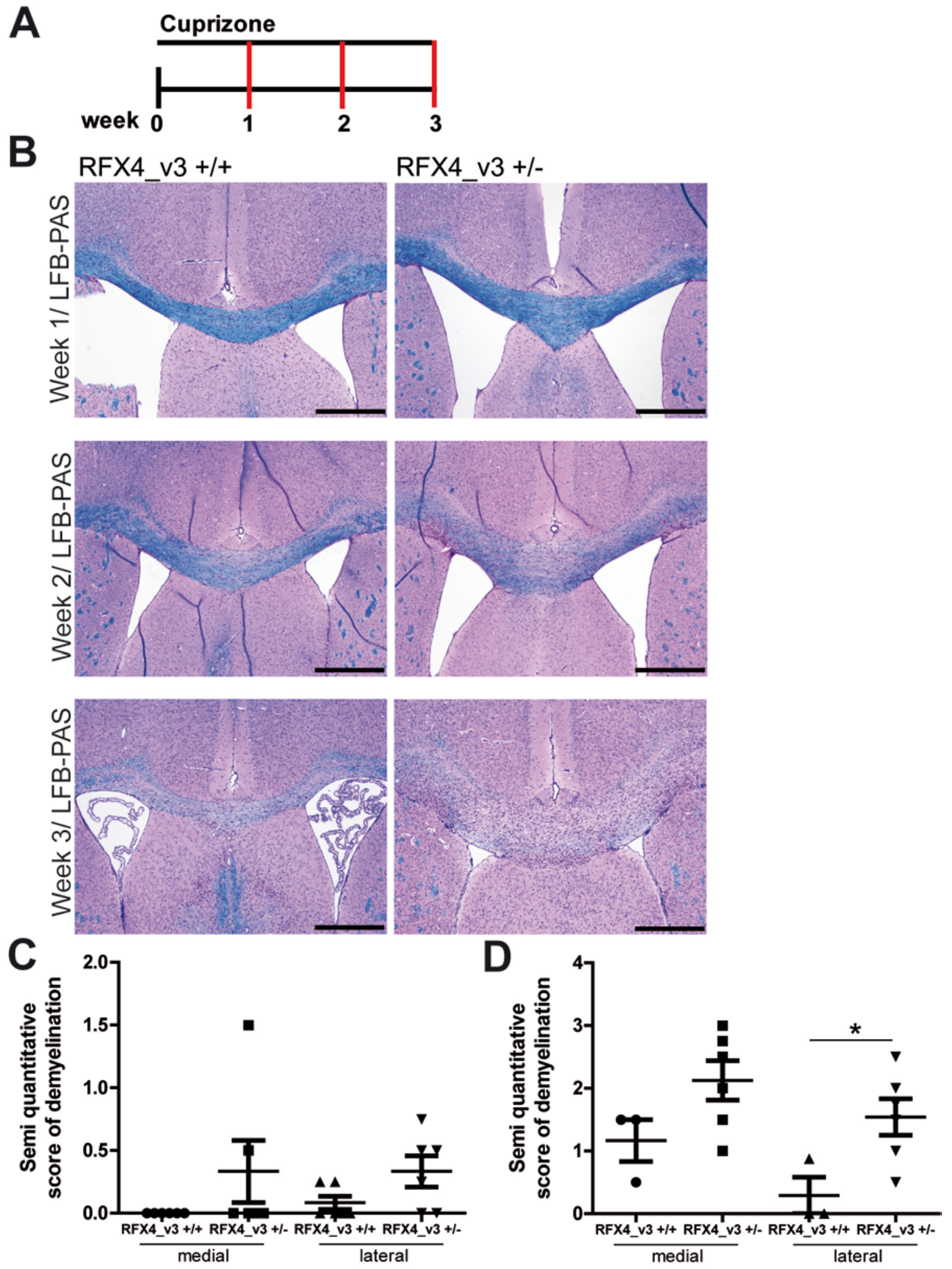

Figure 25: RFX4_v3 deficiency accelerates demyelination in vivo.

(A) Schematic representation of experimental layout. Time course analysis of demyelination was performed after 1, 2 or 3 weeks of cuprizone ingestion. (B) LFB-PAS histochemistry of representative sections from RFX4_v3 +/+ and RFX4_v3 +/- mice after indicated weeks of cuprizone treatment. (C-D) Quantification of demyelination by scoring LFB-PAS in corpus callosum medial and lateral after (C) 2 
weeks and (D) 3 weeks of cuprizone ingestion. Score of 0 represents complete myelination and score of 3 represents complete demyelination ( ${ }^{*} p<0,05$, Mann Whitney test). Data are presented as mean \pm SEM. Scale bar $=500 \mu \mathrm{m}$.

A time course experiment was performed to analyze demyelination after 1, 2 and 3 weeks of cuprizone ingestion (Figure $25 \mathrm{~A}$ ). The extent of demyelination in sections from RFX4_v3 +/+, RFX4_v3 +/- mice was examined by scoring the extent of demyelination based on LFB-PAS staining. After 1 week of cuprizone treatment the myelin in both RFX4_v3 deficient mice and control mice appeared to be intact (Figure $25 \mathrm{~B}$ ). First signs of demyelination were observed after 2 weeks of cuprizone ingestion with a higher score of demyelination in RFX4 deficient mice compared to wild type mice. The difference was more prominent after week 3 with a significant difference in the lateral part of the corpus callosum (score lateral RFX4_v3 +/- $1.5 \pm 0.3$ vs. RFX4_v3 +/+ $0.3 \pm 0.3,{ }^{*} p<0.05$ ). 


\subsection{Astrocyte specific RFX4_v3 deficiency has no influence on the density of} activated GFAP positive astrocytes in the corpus callosum.

A
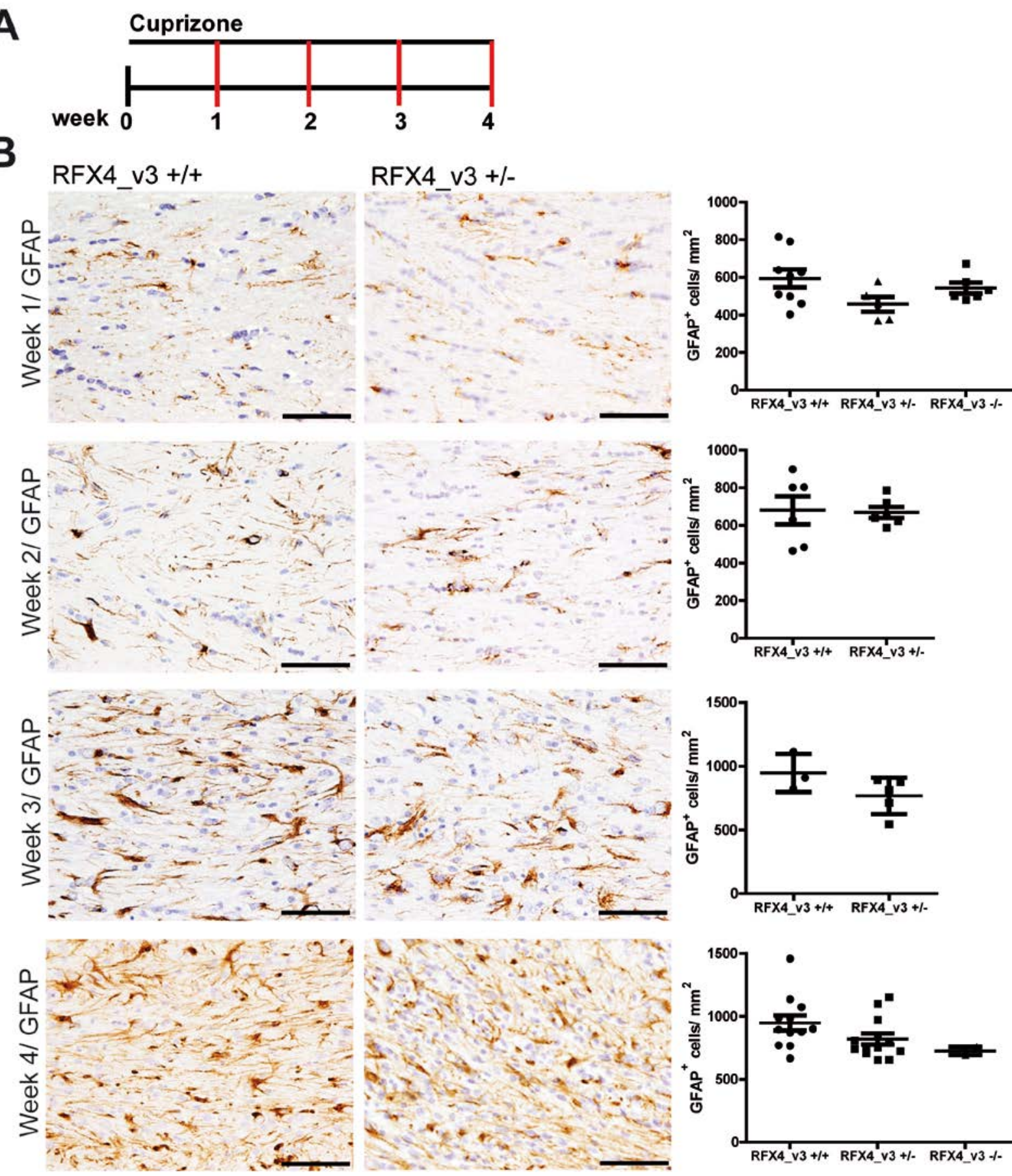

Figure 26: Astrocyte-specific RFX4_v3 deficiency has no influence on the density of activated GFAP-positive astrocytes in the corpus callosum.

(A) Schematic representation of experimental layout. Time course analysis of astrocyte proliferation after 1, 2, 3 or 4 weeks of cuprizone ingestion. (B) Left panels: Representative brain sections of the medial corpus callosum of wild type and RFX4_v3 conditional knockout animals were stained for GFAP after 1-4 weeks of cuprizone treatment. Right panels: Quantification of GFAP positive cells ( $p>0.05$, one way ANOVA with Bonferroni post test (week 1), Kruskal-Wallis test with Dunn's post test (week 4), Mann Whitney test (weeks 2 and 3). Data are presented as mean \pm SEM. Scale bar $=50 \mu \mathrm{m}$ 
It was reported that cuprizone intoxication leads to hyperplasia and an increase in numbers of astroglia, which starts from the second to third week and is most pronounced in the fourth week of cuprizone treatment (Hiremath et al., 1998). Hence I asked whether similar results can be obtained in response to RFX4_v3 inhibition in astrocytes.

Therefore a time course experiment was performed to evaluate the density of GFAP positive astrocytes in the corpus callosum of wild type and RFX4 deficient mice after 1, 2, 3 and 4 weeks of cuprizone diet (Figure 26 A). GFAP immunohistochemistry was used to assess the number of activated astrocytes in brain sections from wild type and RFX4_v3 conditional knockout animals. The densities of activated GFAP positive astrocytes in the corpus callosum were comparable in RFX4 conditional knockout and wild type animals at all time points (Figure $26 \mathrm{~B}$ ). In addition the morphology of astrocytes was comparable until week 3. RFX4_v3 conditional knockout mice showed a slight change in the morphology of the astrocytes after week 4. The astrocytes appeared to be less dense compared to wild type animals, presumably due to the high cellularity in the corpus callosum of RFX4 +/- animals compared to wild type animals, which may leads to a displacement of the astrocytes (Figure $26 \mathrm{~B}$ ). 


\subsection{Astrocyte specific RFX4_v3 deletion leads to accelerated microglia/ macrophage infiltration into the corpus callosum}
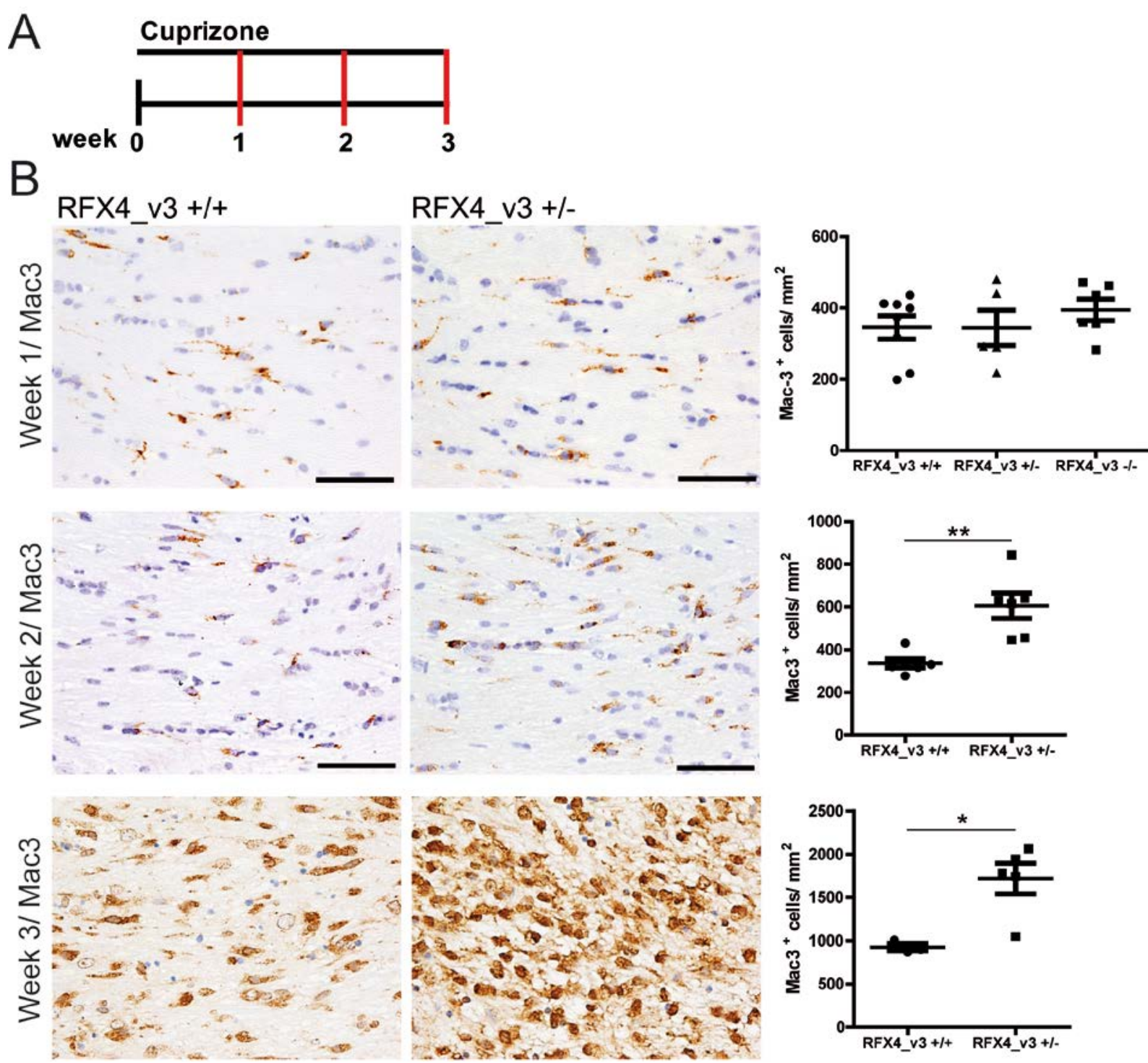

Figure 27: Astrocyte-specific RFX4_v3 deletion leads to accelerated microglia infiltration into the corpus callosum.

(A) Schematic representation of experimental layout. Time course analysis of Mac3-positive phagocytes in the corpus callosum of wild type and RFX4 deficient mice after 1, 2 or 3 weeks of cuprizone diet. (B) Left panels: Mac3 immunostaining of brain sections at given time points after cuprizone treatment. Representative sections are shown. Right panels: Quantification of Mac3-positive cells for each week respectively ( ${ }^{*} p<0.01$, unpaired $t$ test $(B$, week 2$)$, ${ }^{*} p<0.05$, Mann Whitney test $(B$, week 3), $p>0,05$, one way ANOVA with Bonferroni post test (B, week 1$)$ ). Data are presented as mean \pm SEM. Scale bar $=50 \mu \mathrm{m}$.

Due to the considerably enhanced infiltration of the corpus callosum by microglia/macrophage after four weeks of cuprizone challenge, I was interested to study 
the course of microglia activation during the process of demyelination in RFX4_v3 deficient mice compared to control mice.

Therefore a time course experiment was set up to evaluate the density of Mac3 positive microglia in the corpus callosum of wild type and RFX4 deficient mice 1, 2, and 3 weeks after cuprizone ingestion (Figure $27 \mathrm{~A}$ ). The number of activated phagocytes was assessed by immunostaining sections from wild type and RFX4_v3 conditional knockout animals for Mac3. The numbers of Mac3 positive stained microglia/ macrophages were comparable after 1 week of treatment between wild type and RFX4_v3 conditional knockout mice. However, RFX4_v3 deletion led to a faster phagocyte infiltration into the corpus callosum after 2 and 3 weeks with significantly higher numbers of Mac3 positive stained phagocytes in the corpus callosum (week 2 RFX4_v3 +/-: $605 \pm 60$ vs. RFX4_v3 $+/+: 336 \pm 21$ positive Mac3 stained cells per $\mathrm{mm}^{2}$, ${ }^{\star *} \mathrm{p}<0.01$ ), (week 3 RFX4_v3 +/-: 1717 \pm 176 vs. RFX4_v3 +/+: $926 \pm 42$ positive Mac3 stained cells per $\left.\mathrm{mm}^{2},{ }^{*} \mathrm{p}<0.05\right)$.

The morphology of microglia cells in RFX4_v3 deficient mice and control mice was comparable at all time points. In the first and second week the microglia cells appeared to be ramified, while in week 3 the microglia cells appeared to be larger and obtained an amoeboid morphology.

\subsubsection{Deletion of RFX4_v3 in astrocytes increases oligodendrocyte apoptosis and increased mRNA levels of cytokine and chemokines in the corpus callosum}

The time course experiment demonstrated that the first differences in demyelination and microgliosis appear after two weeks of cuprizone challenge in RFX4_v3 deficient mice compared to wild type mice. I concluded that significant differences may take place as early as after 1 week of cuprizone diet in RFX4_v3 deficient mice compared to wild type mice.

Accordingly I decided to treat RFX4_v3 deficient mice for one 1 week with a $0.25 \%$ cuprizone diet and further investigated oligodendroglial apoptosis, which is typically observed at this time point (Figure 28). Furthermore I measured chemokine and cytokine mRNA levels in the corpus callosum of RFX4_v3 deficient mice, which contribute to microglia recruitment and oligodendrocyte toxicity in the cuprizone mouse model (Figure 29). 


\subsection{Increased oligodendroglial apoptosis under loss of RFX4_v3 in astrocytes}

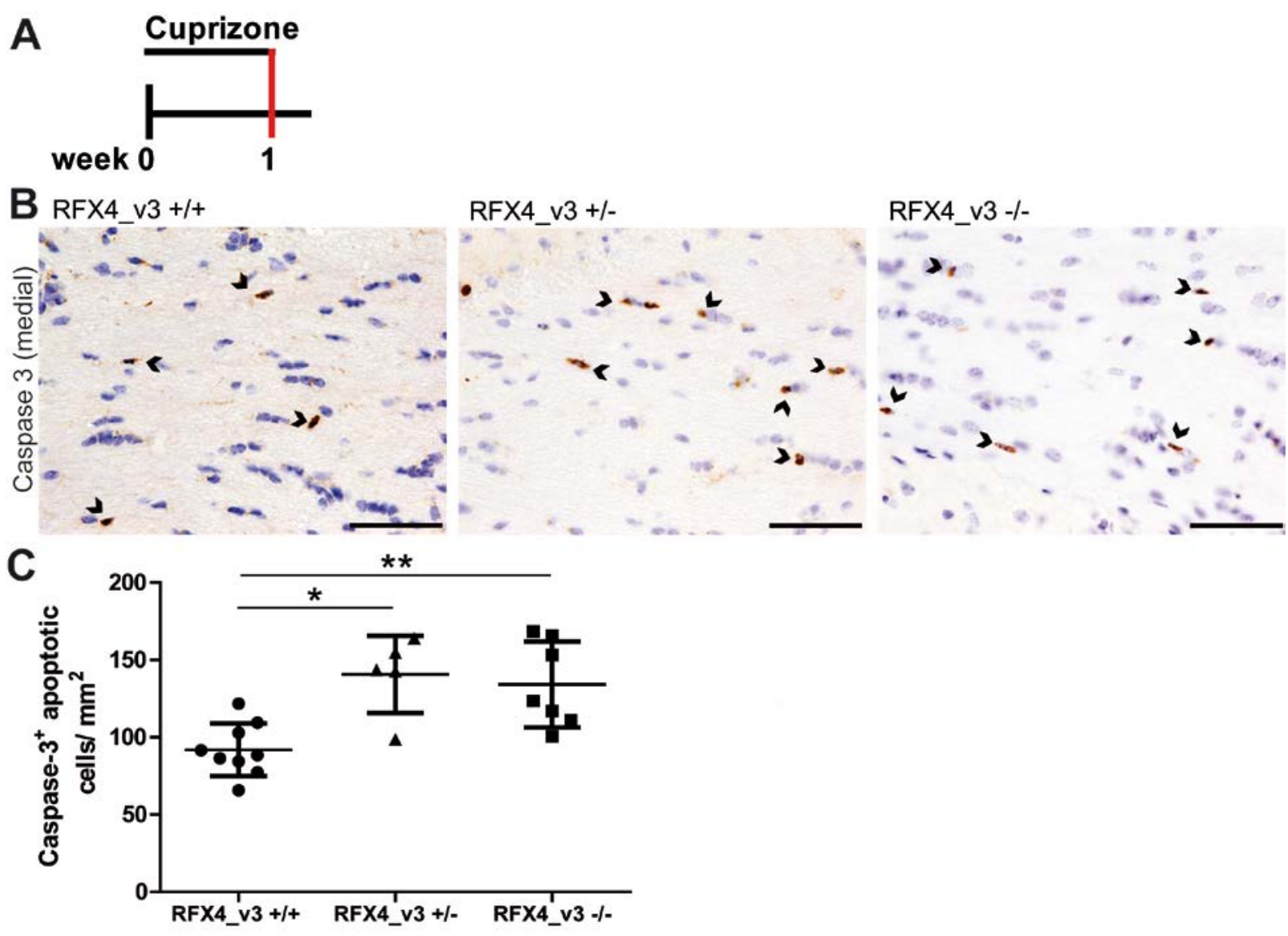

Figure 28: Increased oligodendroglial apoptosis in RFX4_v3 deficient mice.

(A) Experimental layout. RFX4_v3 +/+, RFX4_v3 +/- and RFX4_v3 -/- mice were treated for one week with $0.25 \%$ cuprizone diet. (B) Apoptotic cells were stained with caspase 3 using brain sections of indicated mice. Representative images are shown. (C) Quantification of caspase 3-positive cells in corpora callosa of indicated mice ( ${ }^{*} p<0,05,{ }^{*} p<0,01$ one way ANOVA with Bonferroni post test). Data are presented as mean \pm SEM. Scale bars $=50 \mu \mathrm{m}$

Evaluation of apoptotic cells took place after one week of $0.25 \%$ cuprizone treatment. Sections of RFX4_v3 +/+, RFX4_v3 +/- and RFX4_v3 -/- mice were stained for caspase 3 , a marker for cells undergoing apoptosis.

Compared to wild type animals, RFX4 deficient mice show more apoptotic cells in the corpus callosum (Figure 28). Quantitative analysis revealed a significant difference of RFX4_v3 deficient mice compared to wild type mice (RFX4_v3 +/- $140 \pm 11$ RFX4_v3 -/$134 \pm 10$ vs. RFX4_v3 +/+ $92 \pm 6$ positive caspase 3 stained cells per $\mathrm{mm}^{2},{ }^{*} \mathrm{p}<0.01$ $\left.{ }^{* *} \mathrm{p}<0.01\right)$. 
3.2.2.4.2 Inhibition of astrocytic RFX4_v3 substantially increases transcripts for proinflammatory cytokines and chemokines in response to cuprizone treatment in the corpus callosum

A RFX4

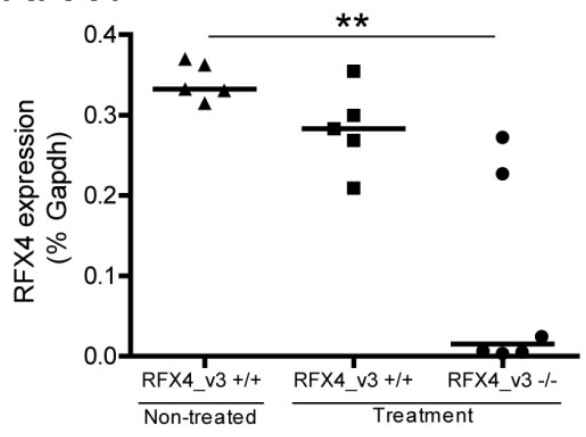

C CCL2

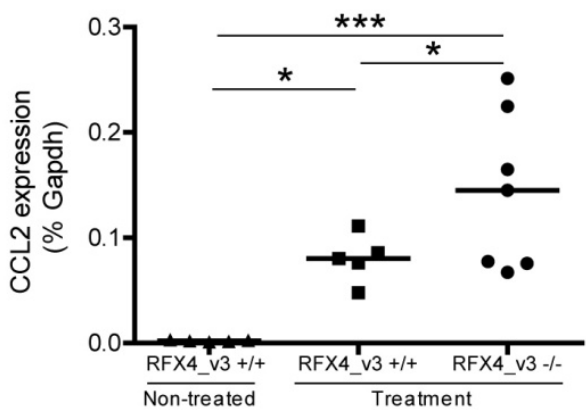

CXCL10

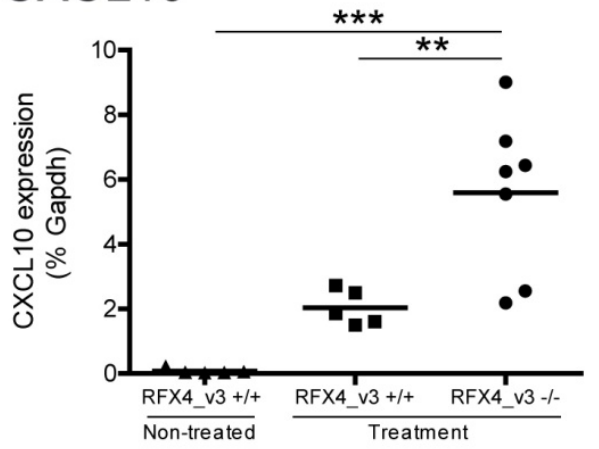

CCL3

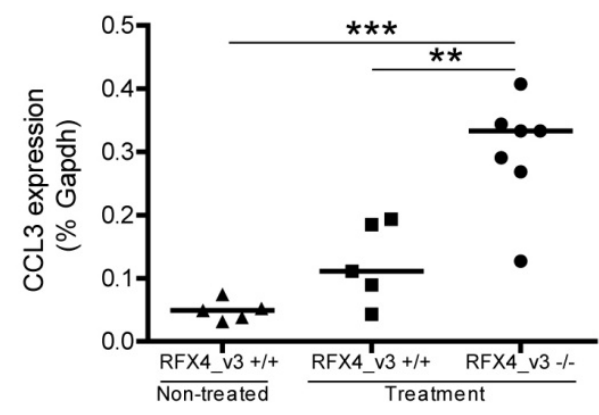

B GFAP

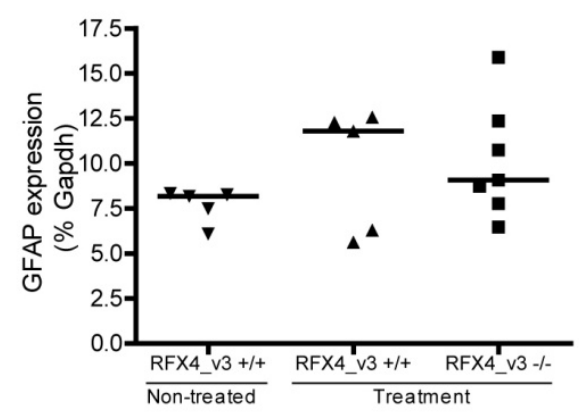

IL-1ß

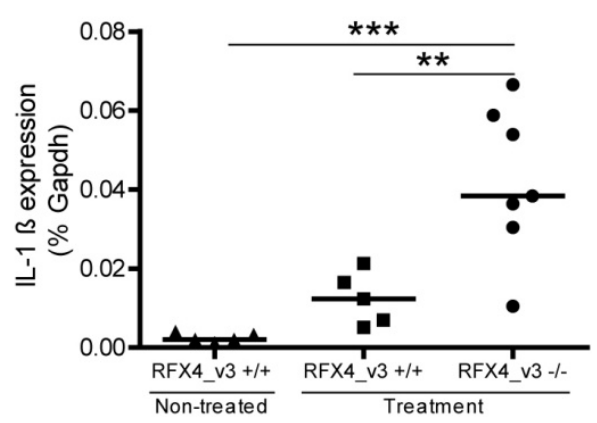

TNF- $\alpha$

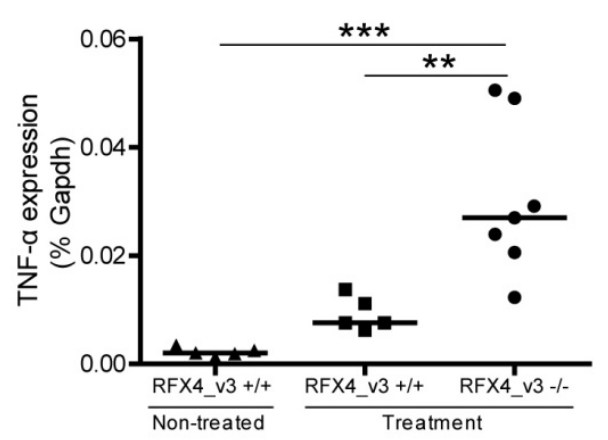

CXCL12

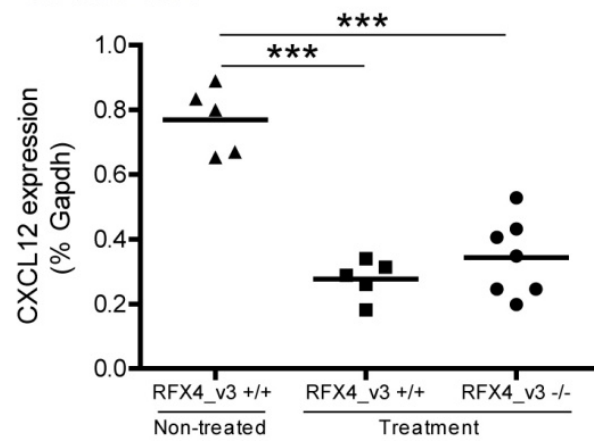

Figure 29: Loss of RFX4_v3 increases the pro-inflammatory response in the corpus callosum. Transcript levels were measured using quantitative real-time PCR for (A) RFX4,(B) GFAP and (C) cytokines/ chemokines in RNA isolated from freshly prepared corpus callosum of untreated and 
cuprizone treated RFX4_v3 +/+ and RFX4_v3 -/- mice. One representative experiment is shown. For each gene the mean of each group is indicated. RFX4: ${ }^{\star *} p<0,01$, Kruskal-Wallis test with Dunn's post test. GFAP: $p>0,05$, one way ANOVA with Bonferroni post test. CXCL10: ${ }^{* *} p<0.01$, ${ }^{* \star} p<0.001$, IL$1 \beta$ : ** $p<0.01,{ }^{* \star *} p<0.001, C C L 2:{ }^{*} p<0.05$, ${ }^{* \star *} p<0.001$, TNF- $\alpha:{ }^{* \star} p<0.01,{ }^{* \star *} p<0.001$, CCL3: ** $p<0.01,{ }^{* \star *} p<0.001, C X C L 12:{ }^{* *} p<0.001$, one way ANOVA with Bonferroni post test.

To measure transcript levels after 1 week of cuprizone intoxication quantitative realtime PCR was used. The transcript levels of RFX4 (Figure 29 A), GFAP (Figure 29 B) and cytokines/ chemokines (Figure $29 \mathrm{C}$ ) from samples isolated from freshly prepared corpus callosum of untreated and cuprizone treated RFX4_v3 +/+, and RFX4_v3 -/- mice were measured (Figure 29).

Cuprizone treatment itself had no significant influence on mRNA levels of RFX4 and GFAP after 1 week of cuprizone challenge. However there is a significant difference in RFX4 mRNA levels between wild type and RFX4_v3 -/- under similar mRNA levels of GFAP in the corpus callosum (Figure 29 A, B). RFX4 transcript levels of RFX4_v3 -/mice were decreased about 4 -fold $\left({ }^{\star *} p<0.01\right)$ compared to wild type animals (Figure 29 A).

Cuprizone treatment itself had an impact on measured cytokine and chemokine levels (Figure $29 \mathrm{C}$ ). Transcript level of CCL2 was significantly increased (about 40-fold ${ }^{*} p<0.05$ ), whereas transcript levels of CXCL12 (about 3 -fold ${ }^{* * *} p<0.001$ ) were significantly decreased (Figure $29 \mathrm{C}$ ). Transcript levels of CXCL10 (about 27 fold, p> 0.05 ) and CCL3 (about 3 fold, p> 0.05) increased, but not significantly (Figure $29 \mathrm{C}$ ).

RFX4_v3 deficiency in astrocytes significantly enhanced transcript levels of all measured cytokines and chemokines, except CXCL12, after 1 week cuprizone challenge (Figure $29 \mathrm{C}$ ). The mRNA levels of CCL2 were significantly increased at about 2-fold for RFX4_v3 -/- mice $\left({ }^{*} p<0.05\right)$ compared to wild type. Transcript levels of IL-1 $\beta$ were significantly increased at about 4-fold for RFX4_v3 -/- mice $\left({ }^{* *} p<0.01\right)$ compared to wild type. CXCL10 mRNA levels were significantly increased at approximately 3-fold for RFX4_v3 -/- mice $\left({ }^{\star \star} p<0.01\right)$ compared to wild type. The transcript levels of TNF- $\alpha$ were significantly increased at approximately 3-fold for RFX4_v3 -/- mice (**p<0.01), compared to wild type. The same was measured for CCL3 with significant increased mRNA levels at approximately 3-fold for RFX4_v3 -/- mice $\left({ }^{* *} p<0.01\right)$ compared to wild type mice. 
4 Discussion

\subsection{Part 1: Characterization of the GFAP cell specific RFX4 conditional knockout mice}

\subsubsection{GFAP specific loss of RFX4 leads to congenital hydrocephalus}

Hydrocephalus is characterized by an abnormal accumulation of CSF in the brain. CSF is produced and secreted by the epithelial cells of the choroid plexus (Ortloff et al., 2013). It circulates through the ventricular system from the lateral to the third ventricle and then via the cerebral aqueduct into the fourth ventricle. From the fourth ventricle the CSF flows via the median aperture of Magendie and the lateral apertures of Luschka into the subarachnoid space before being drained into the venous circulation. Hydrocephalus can be caused by overproduction of CSF, a failure to drain the CSF at the subarachnoid space or reduced reabsorption of the CSF (Pérez-Fígares et al., 2001; Linninger et al., 2009). However, it has been reported that the main cause of congenital hydrocephalus is the obstruction of CSF flow through the narrow Sylvian aqueduct. The Sylvian aqueduct is located between the third and fourth ventricles (Pérez-Fígares et al., 2001; Huh et al., 2009). It is suggested that genetic factors play a role in the pathogenesis of hydrocephalus. So far only one gene, the X-linked L1CAM, has been identified in humans. (Sztriha et al., 2002). This gene is responsible for about $5 \%$ of the congenital cases with genetic cause (Bruni et al., 1988a; Zhang et al., 2006b). The analysis of genetically modified animal models has led to the identification of more than 15 genes resulting in hydrocephalus in rodents (Rolf et al., 2001; Blackshear et al., 2003; Baas et al., 2006; Dietrich et al., 2009; Huh et al., 2009; Benadiba et al., 2012).

In 2003, Blackshear and colleagues described that RFX4_v3 is crucial for early brain development and that its absence leads to congenital hydrocephalus (Blackshear et al., 2003). Disruption of both RFX4_v3 alleles leads to severe brain deformation with failure of dorsal midline formation. All pups homozygous for RFX4_v3 died shortly before or after birth (Blackshear et al., 2003). RFX4_v3 +/- mice survived, but suffered from congenital hydrocephalus associated with hypoplasia or absence of the SCO. The fact that lack of one allele already leads to congenital hydrocephalus was explained by either autosomal dominance or gene dosage effects (Blackshear et al., 2003; Zhang et al., 2007).

In this study I demonstrate that GFAP cell specific RFX4 conditional knockout mice exhibit a very similar phenotype. GFAP cre driven cell specific disruption of RFX4 led to agenesis of the SCO and congenital hydrocephalus resulting in death in young adulthood. 
The RFX4 flox/flox strain contains LoxP sites flanking the exon 4 of the RFX4 gene. RFX4 conditional knockout mice were generated by crossing the RFX4 flox/flox strain with a GFAP driven Cre transgenic strain, which results in RFX4 gene inactivation by deletion the exon 4 in vivo. Three variants of RFX4 have been described in mice, which includes variants 1, 2 and 3. Only RFX4_v2 and RFX4_v3 include the exon 4 in their sequence. Using transcript specific probes Blackshear and colleagues determined, that RFX4_v2 as well as RFX4_v1 are expressed in the testis, but they could not observe hybridization for either variant in the fetal and adult brain (Blackshear et al., 2003). However, RFX4_v3 has been identified in the fetal and adult brain and was not found in the testis (Blackshear et al., 2003; Matsushita et al., 2005). Therefore and due to the phenotype of the GFAP cell specific RFX4 conditional knockout mice I conclude that RFX4 variant 3 is deleted in our strain. In the following parts of my thesis the GFAP cell specific RFX4 conditional knockout mice are therefore termed GFAP cell specific RFX4_v3 conditional knockout mice.

The mortality rate at an age of 6 weeks was about $20 \%$ for heterozygous RFX4_v3 conditional knockout mice, while homozygous RFX4_v3 conditional knockout mice died at a rate of about $50 \%$ at the same age. This implies that the phenotype of RFX4_v3 conditional knockout mice is also due to either autosomal dominance or more likely to gene dosage effects. This was further confirmed by the fact that RFX4_v3 +/- conditional mice had a moderate to marked hydrocephalus, whereas the majority of the homozygous animals analyzed showed a marked to severe hydrocephalus. In newborn transgenic mice hydrocephalus was already present at birth, indicating that the hydrocephalus is congenital. This suggested a blockage in the aqueduct of Sylvius that has also been reported for RFX4_v3 transgenic animals (Blackshear et al., 2003).

\subsubsection{Hydrocephalus in RFX4_v3 conditional knockout mice is caused by SCO hypoplasia}

As mentioned above the primary cause of congenital hydrocephalus is stenosis or narrowing of the Sylvian aqueduct (Perez Figares, 2001). The etiopathogenesis of aqueductal stenosis is not well understood, but it has been reported that maldevelopment and resulting dysfunction of the SCO is involved in the genesis of aqueductal stenosis (Vio et al., 2000). The SCO also known as Reissners fiber complex is a small brain gland of the circumventricular system (Vio et al, 2000). The SCO is built by secretory ependymal cells, which secrete glycoproteins into the ventricles, where they aggregate and form the so called Reissner fiber. These aggregates grow at a constant rate and extend along the aqueduct, fourth ventricle and central canal of the spinal cord (Vio et al., 
2000). The secretion of glycoproteins into the rostral end of the cerebral aqueduct may prevent the closure of the cerebral aqueduct. This would hinder stenosis of the aqueduct and prevent hydrocephalus formation (Vio et al, 2000). A direct prove for this theory was given by the fact that immunizing pregnant rat with RF glycoproteins induced SCO defects, stenosis and hydrocephalus (Sara Rodriguez et al, 1999).

RFX4 variant 3 is described to play an important role for the development of the SCO (Blackshear et al., 2003). Hypoplasia or absence of the SCO in RFX4_v3 +/- mice was described as the main cause for the hydrocephalus (Blackshear et al., 2003).

In our study, the SCO was present in all analyzed RFX4_v3 conditional knockout animals. However, all symptomatic mice exhibited SCO hypoplasia. RFX4_v3 conditionally deficient mice showed a reduced number of SCO ependymocytes. The remaining cells were flattened, unstructured and their apical borders no longer extended into the lateral ventricle. Thus the specialized ependymocytes of the SCO were either absent or inappropriately differentiated in RFX4_v3 conditional knockout mice.

Moreover, residual ependymal cells of the SCO showed reduced immunoreactivity or were not immunoreactive with anti-Reissner's fiber serum (AFRU), implicating a dysfunctional glycoprotein secretion.

This observation is consistent with the findings of Blackshear and colleagues and supports the hypothesis that hydrocephalus in conditional knockout animals is caused by maldevelopment of the SCO.

The SCO originates from neuroepithelial cells that line the lumen of the dorsocaudal aspect of the diencephalon. These epithelial precursors of the SCO differentiate to specialized secretory ependymocytes in response to the induction of Wnt and Bmp signals (Liem et al., 1995; Liem et al., 1997). Blackshear and colleagues showed by in situ hybridization that RFX4_v3 is expressed in early development. From E12.5 to birth, levels of RFX4_v3 mRNA were detected in the neuroepithelium and the ependyma of the neural tube. In addition from E14.5 to birth RFX4_v3 transcripts were found in the ependymocytes of the developing SCO (Blackshear et al., 2003).

In our conditional knockout mice, RFX4_v3 is deleted under control of the GFAP promoter. GFAP is the major intermediate filament protein of mature astrocytes. Sousa and colleagues described that the expression of GFAP is regulated under developmental and pathological conditions (Sousa, Vivian de Oliveira et al., 2004). GFAP is also expressed in neural progenitors like the neuroepithelium during embryogenesis and in nearly all adult neural stem cells (Garcia, A Denise R et al., 2004). It has been reported that in adult rat and mouse, the circumventricular organs are rich in GFAP positive cells (Bennett et al., 2009). Moreover an immunohistochemical study of rat SCO- 
ependymocytes illustrates that these cells express GFAP from E19 until P3 (Chouaf et al., 1991). For adult RFX4_v3 conditional knockout mice it can be assumed that RFX4_v3 will be absent in astrocytes and neural stem cells. During embryogenesis RFX4_v3 will be deleted in neural progenitors including the neuroepithelium (Goldman, 2003; Garcia, A Denise R et al., 2004), suggesting that deletion of RFX4_v3 in conditional and transgenic mice which leads to interruption of RFX4_v3 is in many aspects congruent during embryogenesis. This implicates that similar processes in response to the development of the hydrocephalus occur.

\subsubsection{Regulation of developmental factors by RFX4_v3 impairs SCO formation and function}

Maldevelopment of the SCO is associated with a number of genetic mutations including the Wnt and Bmp signaling pathways. These morphogens influence the establishment of dorsolateral patterning of the CNS and the development and function of the SCO and the ventricular ependymal cells (Liem et al., 1995; Liem et al., 1997). A transcriptional repressor of these pathways is the Msx1 protein. Homozygous Msx1 knockout mice were not able to maintain Wnt1 and Bmp6 in the dorsal midline of P1 mice (Bach, 2003). The loss of morphogens reduced the transcript levels of the cell fate markers paired box 6 and 7 (Pax6/7). As a consequence Msx1 mutants developed a hydrocephalus and had a dysfunctional SCO together with neuroependymal denudation (Bach, 2003; Ramos et al., 2004). In addition to that Wnt1 and Pax6 mutants revealed similar hydrocephalic or SCO phenotypes (Louvi A and Wassef M, 2000; Estivill-Torrús et al., 2001). Similarly, 2009 Dietrich and co-workers published that inactivation of Huntington's disease gene in Wnt1 cell lineages display reduced growth, dismorphic SCO and congenital hydrocephalus as reflected by expansion of the lateral ventricle and loss of cortical tissue (Dietrich et al., 2009). From these studies, Wnt and Bmp signaling seem to be important regulatory mechanisms necessary for the correct differentiation of ependymocytes of the SCO and for preventing the initiation of hydrocephalus formation. Two members of the RFX family RFX3 and RFX4_v3 are found in the dorsal neural tube and seem to be crucial for regular SCO development (Blackshear et al., 2003; Baas et al., 2006). Homozygous RFX4_v3 complete knockout mice were embryonically lethal due to ruinous midline defects (Blackshear et al., 2003). These defects were accompanied by down regulation of Msx2 and a loss of Wnt7a and Wnt7b in the cortical hem at E12.5 (Blackshear et al., 2003). Furthermore a microarray of E10.5 heads of RFX4_v3 -/- mice identified components of Wnt and Bmp signaling as potential targets for RFX4_v3 (Blackshear et al., 2003; Zhang et al., 2006a). 
Because the Wnt and Bmp signaling pathways play an important role in early and late stages of brain development (Zhang et al., 2007) I investigated these genes in a qPCR study of PO astrocytes from conditional knockout mice. The study revealed that genes implicated in Wnt and Bmp signaling pathways are regulated in RFX4_v3 conditional knockout mice. I was able to show that mRNA levels of Wnt3, Rspondin and Frizzeled10 are reduced at the time of birth as was shown before at E10.5 in RFX4_v3 -/- mice. In addition to established factors regulated by RFX4_v3, I could show that ß-catenin, an important regulator of Wnt signaling, is down-regulated at P0. This gene was found to be crucial for proper midbrain development. Conditional mutation of $ß$-catenin led to hydrocephalus (Ohtoshi, 2008). Other transcripts quantified like IGF1, vitronectin and CX3CL1 confirmed and supported our hypothesis that genes in conditional RFX4_v3 knockout animals are similarly modulated as in the complete RFX4_v3 knockout mice.

Although not all genes studied were detectable at P0 (i.e. Ms $\times 3$, Wnt3a) these findings imply that RFX4_v3 deleted astrocytes regulate early morphogens that are relevant for dorsal midline structuring including the development of the SCO. Additionally this supports the idea that hydrocephalus in our RFX4 conditional knockout strain is mainly caused by SCO agenesis and resulting stenosis of the aqueduct, which is in line with the work of Blackshear, 2003 and Zhang, 2006.

In summary, our results indicate that astrocytic and ependymal RFX4_v3 is highly relevant for early brain development and the pathogenesis of congenital hydrocephalus in rodents.

Congenital hydrocephalus in humans occurs with an incidence of $0.1-0.3 \%$ of live births (Perez Figares et al., 2001). So far, only the gene L1CAM has been linked to congenital hydrocephalus in humans (Sztriha et al., 2002). Further research is required to investigate if different expression levels of RFX4_v3 are involved in the pathogenesis of human congenital hydrocephalus. 


\subsection{Part 2: The role of astrocytic RFX4_v3 in demyelination}

\subsubsection{RFX4 is produced by astrocytes and up-regulated in MS lesions}

MS is a chronic demyelinating, inflammatory autoimmune disease, typically affecting multiple regions within the CNS. MS lesions are characterized by demyelination, inflammation, axonal loss and gliosis (Brück, 2005; Milo and Kahana, 2010; Lassmann, 2011; Kutzelnigg and Lassmann, 2014). The mechanisms leading to tissue injury in MS are complex and not fully understood. A specific inflammatory environment, predominantly consisting of pro- and anti-inflammatory molecules, appears to play a central role in regulating CNS inflammation and regeneration. On the one hand, active MS lesions display a mainly pro-inflammatory environment, which probably causes inflammation-associated demyelination. On the other hand at later stages MS lesions can exhibit a more anti-inflammatory and protective environment to support remyelination and neuroregeneration (Kerschensteiner et al., 2003). Different cell types have been described to secrete cytokines, chemokines and neurotoxins, which contribute to the inflammatory milieu. These cell types comprise infiltrating peripheral immune cells like auto-reactive $\mathrm{T}$ cells and macrophages as well as CNS-resident cells like microglia and astrocytes (Nair et al., 2008).

Astrocytes have been deemed to play a secondary role in MS. However, it is now believed that astrocytes play an active role in MS due to their immunomodulatory capacity (Williams et al., 2007; Nair et al., 2008). Astrocytes have been shown to modulate de- and remyelination through the production and secretion of pro- and antiinflammatory cytokines and chemokines (Dong and Benveniste, 2001; Nair et al., 2008). They may promote demyelination by enhancing the immune response by antigen presentation or secretion of chemotactic molecules (Dong and Benveniste, 2001; McFarland and Martin, 2007). Conversely, astrocytes also inhibit aspects of immune response pathways, thereby downmodulating demyelination for example by production of anti-inflammatory cytokines (Schönrock et al., 2000; Valerio, 2002).

Based on Cahoy and colleagues 2008, Dr. Andreas Junker did a quantitative PCR study to measure 96 astrocyte-specific genes in MS lesions to determine astrocyte functions during inflammatory demyelination in MS lesions. This approach revealed RFX4 as a highly up-regulated factor in MS lesions. The work presented here demonstrates that RFX4 is expressed in various cell types of actively demyelinating MS lesions and chronic inactive MS lesions. In addition double IHC of RFX4 and GFAP displayed prominent expression of RFX4 in astrocytes. This confirmed the work of Cahoy and colleagues, which find RFX4 highly enriched in astrocytes (Cahoy et al., 2008).

In humans four main splice variants of RFX4 have been described, but only variant 3 was detected in brain tissue (Matsushita et al., 2005). Likewise, RT PCR with variant 
specific primers (Matsushita et al., 2005) demonstrated that only the mRNA of RFX4_v3 is present in MS lesions.

RFX4 belongs to the protein family of regulatory factor $x$ (RFX), which share a highly conserved winged helix DNA binding domain (Morotomi-Yano et al., 2002). Interestingly, members of the RFX family are known as regulators of immune response (Moreno et al., 1997) and RFX4_V3 modulates the expression of CX3CL1, a chemokine gene which is highly expressed in brain and implicated in MS (Zhang et al., 2006a).

Together, these facts raised the possibility that astrocytic RFX4_v3 may act as regulator of neuroinflammation, thereby influencing de- and remyelination in MS. To further investigate the role of the astrocytic RFX4_v3 in MS, GFAP cell-specific RFX4_v3 conditional knockout mice were used to study de- and remyelination in the cuprizone mouse model.

\subsubsection{RFX4_v3 expression is important for regulation of myelination and activation of phagocytes in the cuprizone mouse model}

\subsubsection{Increased demyelination in the corpus callosum of RFX4_v3 deficient mice after 4 weeks of cuprizone treatment}

To investigate the effects of astrocyte specific RFX4_v3 deletion on demyelination, conditional RFX4_v3 knockout animals and wild type controls were fed with cuprizone for 4 weeks. In this toxic experimental MS model, the copper chelator cuprizone is classically fed for 4 to 6 weeks, which induces a significant and reproducible demyelination in a variety of CNS regions, including the corpus callosum (Matsushima and Morell, 2001; Gudi et al., 2009; Koutsoudaki et al., 2009; Silvestroff et al., 2010, Gudi et al., 2014, 2014). Because of the high mortality rate in the conditional RFX4_v3 -/- knockout mice, the animals were treated with cuprizone for 4 weeks. Demyelination was subsequently analyzed in the corpus callosum.

Compared to wild type, RFX4_v3 deficient mice displayed reduced LFB-PAS and myelin protein staining after 4 weeks of the cuprizone diet. A time course experiment revealed first signs of demyelination after 2 weeks of cuprizone ingestion with a higher score of demyelination in RFX4 deficient mice compared to wild type mice. The difference was even more pronounced after week 3 with a significant difference in the lateral part of the corpus callosum. Additionally, compared to wild type, RFX4_v3 deficient mice showed more axonal damage and a reduced total number of oligodendrocytes in the corpus callosum after 4 weeks of cuprizone treatment. 


\subsubsection{Remyelination in RFX4_v3 deficient mice is delayed}

In the model of cuprizone induced demyelination, acute demyelination is followed by spontaneous remyelination upon cuprizone withdrawal. Typically, remyelination in wild type mice is already marked in the 1 st and 2 nd week after cuprizone withdrawal and is almost complete after 4 weeks (Lindner et al., 2008; Torkildsen et al., 2008). Mason and colleagues reported that remyelination already starts during continued cuprizone ingestion (Mason et al., 2001a). Remyelinated axons are detectable with antibodies against myelin proteins CNPase and MBP (Matsushima and Morell, 2001; Lindner et al., 2008; Gudi et al., 2009) and show pale staining with LFB.

In this study cuprizone withdrawal revealed that RFX4_v3 deficient mice failed to remyelinate in the corpus callosum after week 1 of cuprizone cessation. Very low levels of remyelination were detected by analyzing LFB-PAS and MBP in mutant mice. However semiquantitatively, a significant difference between the RFX4_v3 deficient mice and the control mice was not observed for the myelin protein CNPase. This indicates an active ongoing remyelination process, which was further confirmed by a similar density of remyelinated fibers after 14 days in RFX4_v3 deficient and wild type mice. I thus concluded that remyelination is delayed, but not completely failing in RFX4_v3 deficient mice.

The process of remyelination includes migration, proliferation and differentiation of OPCs and a reduction of microglia/macrophages in the demyelinated corpus callosum (Matsushima and Morell, 2001; Stangel and Hartung, 2002). OPCs start to migrate and proliferate in week 3-5 during cuprizone exposure, while microglia cells remove myelin debris in the corpus callosum (Mason et al., 2000). OPC proliferation is followed by differentiation into mature oligodendrocytes to restore the myelin sheath (Koutsoudaki et al., 2010).

Immunohistochemical analyses were performed to evaluate OPC proliferation and differentiation in RFX4 deficient animals during the period of remyelination. Based on Olig2, a marker for precursor and mature oligodendrocytes, a reduced number of oligodendrocytes in the corpus callosum of RFX4_v3 deficient mice was observed after 4 weeks of cuprizone treatment. However, no significant difference in the density of Olig2positive oligodendrocytes was observed after 7 days of remyelination between RFX4_v3 deficient and wild type mice. Thus, OPC proliferation is occurring in both wild type and RFX4 deficient mice, with a delayed onset in RFX4_v3 deficient mice, which shows that the process of regeneration is active and not failing after 7 days of cuprizone withdrawal. By assessing p25, a marker for mature oligodendrocytes, a significantly lower number of mature oligodendrocytes was observed in the corpus callosum of RFX4_v3 deficient mice demonstrating that Olig2-positive cells in RFX4_v3 deficient mice mainly represent OPCs and not mature oligodendrocytes. The fact that OPC proliferation was delayed and 
remyelination was observed after 14 days of cuprizone withdrawal suggests that OPC differentiation is delayed and inefficient but not failing after 7 days of cuprizone withdrawal.

\subsubsection{Increased phagocyte activation and accumulation during de- and remyelination}

Increased demyelination and delayed remyelination were accompanied by massive microglia/macrophage accumulation in the corpus callosum of RFX4_v3 deficient mice. Microglia cells are the resident immune cells of the CNS. In healthy brains, resting microglia scan the parenchyma for injuries to neurons or macroglia such as oligodendrocytes or astrocytes (Ajami et al., 2007). CNS injuries induce activation of microglia cells (Kettenmann et al., 2011). The resting ramified microglia cells take on an amoeboid-like shape, which is characterized by retracted processes (Aguzzi et al., 2013). In the mouse cuprizone model microglia cells become activated within week 1 and significantly increase during week 3 and 4 to clear myelin debris (Hiremath et al., 1998).

The presented work revealed comparable numbers of Mac3-positive microglia for wild type and RFX4_v3 conditional knockout mice after 1 week of cuprizone treatment. However, in week 2 to 4 RFX4_v3 deficient mice showed significantly higher numbers of Mac3-positive phagocytes. This increase in phagocyte accumulation correlated well with the course of demyelination in RFX4_v3 deficient mice.

When demyelination is complete microglia cells reach a maximal number and then start to decline gradually (Mason et al., 2004). Therefore, phagocyte numbers were investigated during the remyelination phase. After 1 week of remyelination a large number of microglia/macrophages were still present in the corpus callosum of RFX4_v3 deficient mice, while their numbers were significantly reduced in the corpus callosum of wild type mice. However, 2 weeks after cuprizone cessation the density of microglia cells in the corpus callosum of RFX4_v3 deficient and wild type mice was comparable. Given these findings, I propose that the massive infiltration of the corpus callosum by microglia postpones the process of regeneration. The large excess of phagocytes might require more time to decline, which in turn leads to a delay in spontaneous remyelination in RFX4_v3 deficient mice.

These results raised two main questions: What is the reason for the massive microglia/macrophage accumulation and how does RFX4_v3 deficiency in astrocytes contribute to this? The second question is whether microglia/macrophage accumulation is the cause or consequence of increased demyelination and delayed remyelination in RFX4_v3 deficient mice. 


\subsubsection{The blood brain barrier is closed in RFX4_v3 deficient mice.}

The first step to answer these questions was to clarify the origin of the Mac3-positive cells in the corpus callosum of RFX4_v3 deficient mice. Therefore, we investigated the BBB integrity in RFX4_v3 deficient and control mice, as a leaky BBB could indicate and result in enhanced infiltration of phagocytes in the corpus callosum. An important function of astrocytes is maintaining the $\mathrm{BBB}$, which is a selective permeable barrier that separates the circulating blood from the CNS parenchyma. The structure of the BBB consists of capillaries built by endothelial cells, a basement membrane and pericytes which are surrounded by astrocyte end-feet, (Ballabh et al., 2004). As astrocytes are involved in formation of the BBB, we questioned the BBB integrity in mice with astrocytic RFX4_v3 deficiency.

Based on IHC for fibrinogen and CD3 we concluded that the BBB is intact in RFX4_v3 deficient mice during cuprizone-induced demyelination. A positive IHC for the glycoprotein fibrinogen and CD3, a marker for lymphocytes, was not observed, which would indicate BBB disruption.

Previous publications showed that the BBB is closed in the cuprizone mouse model (Kondo et al., 1987; Baker et al., 1991; McMahon et al., 2002). Peripheral monocytes were only present in the brains of cuprizone treated mice when the brain was preconditioned, for example through irradiation (Mildner et al., 2007; Remington et al., 2007). Hence, increased numbers of activated microglia in the brain of cuprizone treated mice originate from local recruitment and proliferation of microglia and not from recruitment of monocytes from the blood. Therefore, the cuprizone model is suitable to study de- and remyelination without a significant influence of the peripheral immune system.

In line with these data, the results of the present work indicate that Mac3-positive cells in RFX4_v3 deficient mice most likely represent microglia and not blood-borne macrophages. This further supports the hypothesis that RFX4_v3 deficiency leads to an increased recruitment, activation and proliferation of microglia.

\subsubsection{RFX4_v3 deficiency causes microglial cell proliferation without stimulation of microglial activation markers}

To further characterize microglia cells of RFX4 deficient mice the cells were analyzed by flow cytometry.

Flow cytometry experiments demonstrated that the CD45-low and CD11b-positive cell population was the most abundant cell population in RFX4 deficient mice. In addition this population increased in absolute numbers after 4 weeks of cuprizone treatment, which was in agreement with elevated numbers of proliferating cells in the corpus callosum of 
RFX4_v3 deficient mice. This leads to the assumption that RFX4_v3 deletion in astrocytes increases recruitment and proliferation of microglia cells.

The data is in line with two previously published studies that reported activation, proliferation and migration of microglia into the corpus callosum in response to primary demyelination by cuprizone intoxication. Both studies demonstrated that after 4.5 or 5 weeks of cuprizone intoxication the CD11b-positive and CD45-low population are the most abundant phagocytes in the demyelinated corpus callosum (Remington et al., 2007; Voss et al., 2012). The CD11b-positive and CD45-low population identifies parenchymal microglia. At this time point only $0.5-7 \%$ are infiltrating monocytes, which showed high expression of CD45 (Remington et al., 2007; Voss et al., 2012).

The next question was whether microglia cells of RFX4_v3 deficient mice only increase in cell number due to recruitment and proliferation or whether they have a more "activated" phenotype thereby causing more severe demyelination and axonal damage. A study of Krauthausen and colleagues showed that CXCR3- deficient mice reveal a milder clinical course accompanied by significantly less microglia activation, which was shown by reduced expression levels of CD45 and CD11b (Krauthausen et al., 2014).

Based on the activation markers described by Remington and co-workers (Remington et al., 2007), microglia cells were characterized in the corpus callosum of RFX4_v3 deficient mice and control mice. No significant differences in the expression levels of CD11b, CD45, CD86, CD80 and MHC class I were observed between RFX4_v3 deficient mice and control mice.

In summary these data indicate that astrocytic RFX4_v3 deficiency leads to increased microglia recruitment and proliferation in the corpus callosum in mice exposed to cuprizone. However, loss of RFX4_v3 does not significantly alter the expression of activation markers on microglia cells during demyelination. Therefore, enhanced demyelination and axonal damage cannot be explained by a more activated microglia phenotype, which could lead to increased phagocytic activity. It is more likely that increased numbers of microglia cells in the corpus callosum contribute to increased demyelination and axonal damage. Although it is not clear at this point whether increased microglia recruitment and proliferation is the cause or consequence of increased demyelination and axonal damage.

Based on results from previous studies two possible explanations could account for increased microglia recruitment and proliferation.

First, it was demonstrated that astrocytes regulate microglia recruitment and proliferation by secretion of chemokines and cytokines (Raasch et al., 2011; Skripuletz et al., 2013). In addition inhibition of nuclear factor kappa B activation in astrocytes resulted 
in reduced demyelination, reduced expression of pro-inflammatory mediators and a significantly diminished glial response (Raasch et al., 2011). This led to the hypothesis that deletion of RFX4_v3 in astrocytes may change the astrocytic phenotype or astrocyte function, in particular the ability of astrocytes to secrete cytokines and chemokines.

Second, it was described that less demyelination in the corpus callosum is associated with less oligodendrocyte apoptosis or a limited loss of oligodendrocytes in the corpus callosum. This results in reduced microglia/ macrophage accumulation ( $\mathrm{Li}$ et al., 2008; Raasch et al., 2011). Therefore, I asked the question whether elevated levels of oligodendrocyte apoptosis contribute to enhanced microglia accumulation in RFX4_v3 deficient mice.

\subsubsection{RFX4_v3 loss in astrocytes modulates chemokine and cytokine transcript levels, thereby attracting phagocytes into the demyelinated corpus callosum}

\subsubsection{Astrocyte specific RFX4_v3 deficiency has no influence on numbers of activated GFAP positive astrocytes in the corpus callosum}

Previous studies indicate that astrocytes play a crucial role in the recruitment of microglia in the cuprizone mouse model. Deletion of $I_{K} B$ kinase 2 in astrocytes was sufficient to protect mice from myelin loss in the cuprizone mouse model (Raasch et al., 2011). In addition Skripuletz and colleagues demonstrated that a loss of astrocytes in GFAP-thymidine kinase transgenic mice reduced removal of damaged myelin and also delayed the remyelination process in the cuprizone mouse model. These effects were combined with a decrease in microglial activation and recruitment (Raasch et al., 2011; Skripuletz et al., 2013). Due to RFX4_v3 loss in astrocytes I hypothesized that a possible malfunction of astrocytes could lead to the observed microglia phenotype in the cuprizone mouse model.

Therefore P0 astrocytes were characterized from RFX4_v3 deficient mice and control mice in vitro. Despite the fact that RFX4 transcripts in P0 astrocytes were significantly reduced, P0 astrocytes from RFX4_v3 deficient mice showed no morphological changes by IHC for GFAP. In addition, P0 astrocytes from RFX4_v3 deficient mice behaved in the same way as wild type astrocytes in a scratch migration assay. Thus, in vitro astrocytes from RFX4_v3 conditional knockout mice did not display obvious morphological and functional deficits.

As the next step astrocytes from RFX4_v3 deficient mice and wild type mice were analyzed in the cuprizone mouse model after 1, 2, 3 and 4 weeks.

In the cuprizone mouse model astrocytes usually increase in number and obtain a changed morphology during demyelination. During the first two weeks of $0.2 \%$ cuprizone treatment the quantity of astrocytes remains on a basal level (Hiremath et al., 1998). 
However, during the third week of cuprizone ingestion, when significant levels of demyelination are present, astrocytes significantly increase in numbers (Hiremath et al., 1998). Astrocytes in untreated mice show few, delicate and short processes, while astrocytes from cuprizone treated mice become hypertrophic with thick processes already during the first 2 weeks (Hiremath et al., 1998). Apart from the typical morphological changes, activated astrocytes express high levels of the intermediate filament glial fibrillary acidic protein (GFAP) (Eng, 1985). Astrocytic proliferation is enhanced in response to the toxin cuprizone, which was demonstrated by double immunostaining with BrdU and GFAP (Hiremath et al., 1998; Gudi et al., 2009; Gudi et al., 2014).

In the RFX4_v3 deficient mice the number of activated GFAP-positive astrocytes in the corpus callosum was comparable to wild type animals at all time points and thus was in line with the literature (Hiremath et al., 1998). In addition the morphology of astrocytes was comparable until week 3. After 4 weeks I observed slight changes in RFX4_v3 deficient mice. At this time point astrocyte density decreased compared to the wild type animals. This is probably due to the high cellularity of the corpus callosum of RFX4_v3 heterozygous animals, which may lead to a displacement of astrocytes. In addition after 1 week the expression level of the astrocytic activation marker GFAP in the corpus callosum was comparable between RFX4_v3 deficient and control mice.

Altogether no substantial differences were found in cell density, morphology, and function between astrocytes from RFX4_v3 deficient and control mice in vitro and in vivo after cuprizone treatment. Thus, microglia recruitment and proliferation in RFX4_v3 deficient mice cannot be explained through changes in astrocyte phenotype or density. However, I assumed that astrocytic functions such as chemokine and cytokine production were modified in RFX4_v3 deficient mice upon cuprizone treatment.

\subsubsection{Inhibition of astrocytic RFX4_v3 substantially increases transcripts of pro-inflammatory cytokines and chemokines in response to cuprizone}

Astrocytes produce a bulk of chemokines in MS (Dong and Benveniste, 2001; Nair et al., 2008). Hence, it was suggested that chemokine secretion is important for demyelination to recruit inflammatory cells to the injured area, such as macrophages and microglia (Babcock et al., 2003; Remington et al., 2007; Bianchi et al., 2011; Qin and Benveniste, 2012).

The present work demonstrates that RFX4_v3 deficient mice have elevated levels of demyelination, oligodendrocyte loss and axonal damage, which are accompanied by massive microglia activation, recruitment and proliferation. Based on the reports discussed above I wondered whether astrocytic RFX4_v3 is involved in regulating the cytokine and chemokine production in astrocytes after cuprizone treatment. Compared to 
wild type mice the first noticeable differences in demyelination and microgliosis in RFX4_v3 deficient mice appeared after two weeks of cuprizone treatment. Hence, I assumed that differences between wild type and RFX4_v3 deficient mice would start to appear after approximately one week of cuprizone treatment. Therefore, mRNA expression levels of the chemokines and cytokines CCL2, CCL3, CXCL10, IL-1ß, TNF- $\alpha$ and CXCL12 were measured at this time point in the corpus callosum of RFX4_v3 deficient and wild type mice. Some of these cytokines and chemokines have been reported in the context of altered astrocyte activation in the cuprizone mouse model. Besides decreased demyelination and reduced microglia cells, $I_{K} B$ kinase 2 deletion in astrocytes additionally reduces astrocytic production of chemokines and cytokines including CCL2, CCL3, CXCL10 and TNF- $\alpha$ (Raasch et al., 2011). Skripuletz and colleagues also demonstrated that reduced microglia recruitment is associated with a reduction of CXCL10 production in mice after astrocyte ablation (Skripuletz et al., 2013).

Compared to wild type mice the mRNA levels of CCL2 in RFX4_v3 deficient mice were strongly up-regulated after 1 week of cuprizone diet. Up-regulated levels of CCL2 mRNA were particularly found during the first week of cuprizone ingestion (McMahon et al., 2001; McMahon et al., 2002; Buschmann et al., 2012). This is in line with the results from cuprizone treated wild type mice compared to untreated wild type mice. In MS CCL2 expression was found in the brain, CSF and blood (Mahad and Ransohoff, 2003). In the cuprizone mouse model and EAE this factor was shown to be produced by astrocytes (Ransohoff et al., 1993; Raasch et al., 2011; Kim et al., 2014). GFAP cell specific CCL2 conditional knockout mice have less severe EAE and show lower numbers of macrophages and $\mathrm{T}$ cells recruited into the white matter of the spinal cord and lower numbers of microglia in both white and gray matter. Finally, reduced axonal loss and demyelination was observed in GFAP cell specific CCL2 conditional knockout mice (Kim et al., 2014). Hayashi and colleagues showed that CCL2 induces chemotaxis of microglia. In addition they reported that LPS, IL-1ß and TNF- $\alpha$ stimulation leads to CCL2 secretion by astrocytes, but does not induce CCL2 secretion by microglia (Hayashi et al., 1995). Based on these results, I deduced that up-regulation of CCL2 in astrocytes with RFX4_v3 deletion might be responsible for microglia activation and attraction to the demyelinated corpus callosum.

Compared to wild type mice also CCL3 was significantly up-regulated in RFX4_v3 conditional knockout mice after 1 week of cuprizone treatment. CCL3 has been reported to be present in the brain of mice after 2 days of cuprizone treatment, with increasing levels until week 4 and a slight decrease after week 5 , which was in line with the data of untreated versus treated wild type mice (McMahon et al., 2001; Raasch et al., 2011; Buschmann et al., 2012; Krauthausen et al., 2014). CCL3 is produced by astrocytes and microglia cells in the cuprizone mouse model as well as in EAE (Nygårdas et al., 2000; 
Raasch et al., 2011). Deletion of this chemokine leads to reduced recruitment of microglia and astrocytes, which results in delayed demyelination in the cuprizone mouse model (McMahon et al., 2001). Studies in EAE with neutralizing antibodies against CCL3 and CCL2 revealed that these chemokines regulate the mononuclear accumulation and infiltration, which enhances the severity of the disease (Karpus et al., 1995; Kennedy et al., 1998). In vitro it was demonstrated that CCL3 influences migration and activation of microglia (Hayashi et al., 1995; Cross and Woodroofe, 2001).

The work from Skripuletz and colleagues implied that CXCL10 is involved in the process of activation and recruitment of microglia and macrophages (Skripuletz et al., 2013). With IHC techniques Skripuletz and colleagues showed that the factor was expressed by reactive astrocytes, but not in reactive microglia (Skripuletz et al., 2013). In the present study CXCL10 was significantly up-regulated in RFX4_v3 -/- compared to wild type mice. Increased levels of CXCL10 were also observed in cuprizone treated wild type mice in contrast to untreated wild type mice, which was in line with previous studies (Skripuletz et al., 2013). CXCL10 expression was found in MS, where the factor was expressed in astrocytes in the lesion border of demyelinated plaques (Tanuma et al., 2006). The authors speculated that the production of CXCL10 and CCL2 by astrocytes activates astrocytes and directs reactive gliosis followed by migration and activation of microglia/macrophage in demyelinated lesions (Tanuma et al., 2006). Astroglial CXCL10 deletion in mice led to milder clinical deficits and reduced acute demyelination in EAE accompanied by a reduced accumulation of CD4-positive lymphocytes in the spinal cord perivascular space, while the overall composition of these cells was unchanged. In addition the IBA1-positive microglia/macrophage accumulation was not affected in these mice (Mills Ko et al., 2014).

By contrast, the chemokine CXCL12 was not regulated by RFX4_v3 deletion. After 1 week of cuprizone treatment the factor was significantly down-regulated in both wild type and RFX4_v3 deficient mice to comparable mRNA levels. Expression of CXCL12 in the cuprizone mouse model was shown at later time points during the cuprizone diet, implicating that astrocytic CXCL12 is not important for early microglia/macrophage attraction, but rather for the process of remyelination as was described by Patel and colleagues (Patel et al., 2012).

Compared to wild type mice the pro-inflammatory cytokines IL-1ß and TNF- $\alpha$ were significantly increased in the corpus callosum of RFX4_v3 deficient mice. Previous studies showed that IL-1ß promotes astrocyte proliferation and is also required for reactive astrogliosis upon CNS injury, which is a typical feature in the cuprizone mouse model (Giulian and Lachman, 1985; Herx and Yong, 2001). The cytokine IL-1ß is mainly produced by microglia and macrophages, but was also found in a subpopulation of astrocytes and has been shown to activate TNF- $\alpha$, IL- 6 and nitric oxide, thereby 
contributing to the inflammatory response (Lee et al., 1993; Mason et al., 2001b). It has been reported that the factor is up-regulated from the first week onwards of cuprizone intoxication, which was confirmed by our data. The factor strongly increased at week 3 and stayed on this level until week 6 (Mason et al., 2001). IL-1ß deletion in the cuprizone mouse model reduced the differentiation of OPCs, which leads to inefficient remyelination (Mason et al., 2001b). The role of IL-1ß during the first week of cuprizone exposure is still unclear, but the factor was described to promote reactive astrogliosis and activation of astrocytic chemokine secretion such as CCL2 in vitro. Hence, in RFX4_v3 deficient mice IL-1ß might have an indirect effect on the activation and recruitment of microglia/macrophages by activating the secretion of other chemokines in the corpus callosum.

The cytokine TNF- $\alpha$ has been described to play a dual role in the cuprizone mouse model. Mice lacking TNF- $\alpha$ showed a decreased loss of oligodendrocytes during the first 3 weeks, but also displayed significantly delayed remyelination. Reduced remyelination was associated with a reduction of the pool of proliferating OPCs and thus a reduced number of mature oligodendrocytes (Arnett et al., 2001). Although TNF- $\alpha$ is produced by microglia cells and astrocytes, Raasch and colleagues showed with laser microdissection that astrocytes regulate the transcript level of TNF- $\alpha$ in the cuprizone mouse model (Raasch et al., 2011). TNF- $\alpha$ is involved in many inflammatory diseases such as MS and is mainly produced by microglia/macrophages in the central nervous system, which has been shown to influence leukocyte activation and invasion (Bradley, 2008).

Altogether, I conclude that after cuprizone treatment of astrocytic RFX4_v3 deficient mice, up-regulated levels of CXCL10, CCL2, CCL3, IL-1ß and TNF- $\alpha$ are involved in the activation and recruitment of microglia/macrophages. The results suggest that the transcription factor RFX4_v3 controls the activation and response of astrocytes through production of chemokines and cytokines, thereby influencing the recruitment of phagocytes. Finally this could contribute to increased demyelination, oligodendrocyte loss and axonal damage in RFX4_v3 deficient mice.

\subsubsection{The transcription factor RFX4_v3 possibly acts as a regulator of cytokine and chemokine production in MS}

In the neuroinflammtory disorder MS RFX4_v3 was found to be up-regulated. The factor showed a prominent expression in the nucleus of astrocytes in active and inactive MS lesions. In addition, it was suggested that RFX4 acts rather as a transcriptional repressor than as a transcriptional activator (Morotomi-Yano et al., 2002; Zhang et al., 2007). This could be an indication for a protective role of RFX4 in the context of neuroinflammation in MS. 
MS lesions are characterized by inflammation, demyelination and axonal damage (Lassmann et al., 1998). It is thought that MS is driven by a T cell mediated autoimmune reaction against myelin proteins (Hohlfeld, 2004). In the context of neuroinflammation, astrocytes have been reported to regulate the infiltration of peripheral pro-inflammatory leukocytes into the CNS (Bush et al., 1999; Voskuhl et al., 2009; Toft-Hansen et al., 2011; Kim et al., 2014). Ablation of reactive astrocytes in adult transgenic mice leads to an increased infiltration of leukocytes including monocytes, macrophages, neutrophils and lymphocytes, failure of the BBB repair and neuronal degeneration (Bush et al., 1999). This was confirmed by Toft-Hansen and co-workers, who showed that inhibition of reactive astrocytosis after initiation of EAE causes increased macrophage, but not $T$ cell infiltration and leads to enhanced severity of EAE (Toft-Hansen et al., 2011). Increased macrophage infiltration was reflected by increased levels of cytokines and chemokines such as TNF- $\alpha$ and CCL2 (Toft-Hansen et al., 2011). A recent publication by Mayo and colleagues showed that regulation of astrocyte activation by glycolipids drives chronic CNS inflammation (Mayo et al., 2014). In this study the authors reported that lactosylceramide (LacCer) and B4GALT6 are up-regulated in the CNS of mice during chronic EAE and MS lesions. In addition, they were able to show that LacCer in astrocytes controls the recruitment and activation of microglia and CNS infiltrating monocytes by regulation of the production of the chemokine CCL2 and granulocyte macrophage colony-stimulating factor (GM-CSF) (Mayo et al., 2014). Downregulation of LacCer synthesis decreased the local CNS innate immunity and neurodegeneration in EAE and also interfered with activation of human astrocytes (Mayo et al., 2014).

All of these studies demonstrate that the production and release of chemokines by astrocytes play an important role in the progression of MS. The data presented in my study, using astrocytic RFX4_v3 deficient mice under cuprizone treatment, further indicate that astrocytes regulate neuroinflammation by controlling chemokine and cytokine release, thereby restricting leukocyte infiltration, which is an indication of a protective role of astrocytes in MS. Hence, our data point to a putative protective role of the transcription factor RFX4_v3 in MS by regulating cytokine and chemokine production and release to restrict neuroinflammation.

\subsubsection{Deletion of RFX4_v3 in astrocytes induces apoptosis of oligodendrocytes}

As mentioned before increased oligodendrocyte apoptosis in the corpus callosum of RFX4_v3 deficient mice versus wild type mice could be an explanation for enhanced microglia activation.

Oligodendrocyte cell death is an early phenomenon during the first three weeks of cuprizone treatment (Hesse et al., 2010; Veto et al., 2010). The mechanism of 
oligodendrocyte apoptosis in response to cuprizone is not well understood. It is thought that selective toxicity of cuprizone to oligodendrocytes through functional disturbances in mitochondria plays an important role during oligodendrocyte apoptosis and, thus, initiation of demyelination (Matsushima and Morell, 2001; Pasquini et al., 2007; Ransohoff, 2012; Bénardais et al., 2013). Within the first 10 days of cuprizone treatment dying oligodendrocytes can be identified by expression of caspase 3 (Hesse et al., 2010).

Indeed, based on caspase $3 \mathrm{IHC}$, oligodendroglial apoptosis proved to be significantly increased in RFX4_v3 deficient mice compared to wild type mice one week after the start of cuprizone treatment.

Several studies describe that reduced demyelination is associated with less oligodendrocyte apoptosis or reduced loss of oligodendrocytes and less microglia activation, which supports the data presented here (Li et al., 2008; Raasch et al., 2011; Brück et al., 2012). Brück and colleagues showed that treatment of wild type and RAG-1deficient mice with Laquinimod (an oral immunoregulatory compound) reduced oligodendrocyte apoptosis, demyelination and microglia activation upon cuprizone feeding (Brück et al., 2012). A similar scenario was observed in a work from Li and colleagues who showed that p53 knockout mice and wild type mice receiving a p53 inhibitor demonstrated less demyelination and reduced numbers of apoptotic oligodendrocytes accompanied by reduced microglia recruitment in the demyelinating corpus callosum.

Furthermore, Raasch and co-workers showed that astrocytic loss of $\mathrm{I}_{\mathrm{K}} \mathrm{B}$ kinase 2 protects mice from myelin loss, which was associated with reduced loss of oligodendrocytes in the corpus callosum. Actually, IHC for Olig2 after four weeks of cuprizone revealed reduced total numbers of oligodendrocytes in the corpus callosum of RFX4_v3 deficient mice, which - considering the data of Raasch and colleagues - could contribute to the increase in demyelination observed in these animals.

In summary, the discussion above indicates that increased injury in the corpus callosum by enhanced oligodendrocyte apoptosis could contribute to increased microglia activation. It is most likely that a combination of both enhanced cytokine and chemokine secretion and increased oligodendrocyte apoptosis in RFX4_v3 deficient mice contributes to the observed microglia phenotype in the cuprizone mouse model, which is in line with the literature (Li et al., 2008; Raasch et al., 2011; Skripuletz et al., 2013). Still, it cannot be completely solved whether enhanced microglia recruitment and proliferation is the cause or consequence of the increased demyelination, reduced oligodendrocytes and increased axonal damage in the corpus callosum of RFX4_v3 deficient mice. 
What could be the reason for increased oligodendrocyte apoptosis in RFX4_v3 deficient mice?

\subsubsection{RFX4_v3 may indirectly influence oligodendrocyte apoptosis by changing the inflammatory environment in the corpus callosum}

The observed increase in oligodendrocyte apoptosis upon cuprizone treatment in RFX4_v3 deficient mice raises the question how apoptosis is triggered in these animals. One option could be a indirect influence of RFX4_v3 on oligodendrocyte apoptosis by upregulation of cytokines and chemokines.

MS lesions are characterized by oligodendrocyte death, axonal damage, gliosis, activation of microglia, and perivascular and parenchymal accumulation of lymphocytes and macrophages (Lassmann et al., 2007; Compston and Coles, 2008). Primary death of oligodendrocytes, is one of the factors suggested to be central to MS (Barnett and Prineas, 2004; Barnett and Sutton, 2006; Locatelli et al., 2012).

One mechanism that may cause injury to oligodendrocytes in MS is signaling via death receptors of the TNF receptor superfamily, such as the receptor FAS (D'Souza, 1996). Death receptors are cell surface receptors, which transmit apoptotic signals by specific ligands like TNF- $\alpha$ and FasL. They play an important role in apoptosis and can activate a caspase cascade within seconds of ligand binding. Members of these receptors have been found on oligodendrocytes in MS and their ligands were shown to induce cytotoxic effects (D'Souza, 1996). In vitro it was shown that exposure of human adult oligodendrocytes to TNF- $\alpha$ leads to oligodendrocyte injury (Souza, 1995). TNF- $\alpha$ induces caspase activation by interaction with specific caspase receptors that regulate the factor $\mathrm{Bcl}-2$, a regulator of cell death. This can lead to mitochondrial dysfunction and OLG apoptosis (Pang et al., 2007). Inhibition of caspase 1 and caspase 3 was shown to protect OLGs against TNF- $\alpha$ - induced apoptosis (Selmaj and Raine, 1988; Louis et al., 1993; Tchélingérian et al., 1995; Hisahara et al., 1997; Cudrici et al., 2006).

In the CNS, TNF- $\alpha$ is primarily produced by microglia and astrocytes, but only microglia cells have been reported to mediate OLG death through TNF- $\alpha$ production (Merrill et al., 1993; Arnett et al., 2001).

We found that TNF- $\alpha$ was significantly up-regulated in RFX4_v3 deficient mice after one week of cuprizone diet. At this time point oligodendrocyte apoptosis was also increased. This implicates involvement of RFX4_v3 in regulation of TNF- $\alpha$ release to control oligodendrocyte cell death in the cuprizone mouse model. A similar scenario is conceivable for MS since RFX4_v3 was up-regulated in various cell types and in particular in astrocytes of MS lesions. It is conceivable that the transcription factor RFX4_v3 controls astrocytes and possibly also microglia through down-regulation of 
cytokine and chemokine levels such as TNF- $\alpha$ to prevent oligodendrocyte death and neurodegeneration in MS lesions.

\subsubsection{Delayed remyelination as a consequence of a dysregulated inflammatory environment by astrocytes in RFX4_v3 conditional knockout mice}

As described in detail above the process of remyelination in RFX4_v3 deficient mice is most likely delayed. Although the number of OPCs in mutant mice was comparable to wild type mice after 7 days of remyelination, the number of mature oligodendrocytes in RFX4_V3 deficient mice was significantly lower than the in wild type mice. The delay in remyelination was associated with enhanced microglia accumulation. I assumed that the increase in microglia cells in the corpus callosum postpones the process of regeneration, including proliferation and differentiation of OPCs into mature oligodendrocytes. It was described that the inflammatory milieu influences the process of remyelination (Franklin, Robin J M and ffrench-Constant, 2008). Therefore it is conceivable that dysregulation of pro-inflammatory cytokines and chemokines by RFX4_v3 in astrocytes cause delayed remyelination.

In MS remyelination does occur, but is inefficient and inadequate (Franklin, Robin J M and ffrench-Constant, 2008). Failure of remyelination is mostly due to a failure or arrest of oligodendroglial differentiation, whereas OPC presence and their migratory capacity are not limiting factors for remyelination in MS (Franklin, Robin J M and ffrench-Constant, 2008). A lack of a supportive cellular environment hinders OPCs to differentiate into mature oligodendrocytes (Franklin, Robin J M, 2002; Franklin, Robin J M and ffrenchConstant, 2008). Therefore, modulation of the inflammatory environment of a lesion could be effective to increase remyelination. In addition to other chemokines TNF- $\alpha$ and IL-1ß were up-regulated one week after cuprizone exposure in RFX4_v3 deficient mice. Genetic deletion of TNF- $\alpha$ and IL-1ß in the mouse cuprizone model significantly reduced remyelination due to an inadequate recruitment and differentiation of OPCs, which indicates that pro- as well as anti-inflammatory factors play a role in remyelination in the mouse cuprizone model (Arnett et al., 2001; Mason et al., 2001b). Olah and colleagues found that in the mouse cuprizone model microglia show a phenotype associated with phagocytosis of myelin debris as well as with recruitment of OPCs through expression of cytokines and chemokines, which provided evidence for the capability of microglia to support remyelination. Therefore, dysregulation of pro-inflammatory cytokines and chemokines by RFX4_v3 in astrocytes may directly or indirectly, through increased microglia cells, postpone remyelination which supports our hypothesis that remyelination is delayed and not failing. In addition, increased phagocytes in the corpus callosum may change the cytokine and chemokine level at later time points in the cuprizone mouse model, and thereby influence the recruitment, proliferation and differentiation of OPCs. 
Altogether the dysregulated pro-inflammatory environment due to RFX4_v3 deficiency in astrocytes may postpone the process of remyelination. I suppose that the increased numbers and prolonged presence of microglia cells are a direct or indirect consequence of the modulated pro-inflammatory environment caused by loss of RFX4_v3, which may impede OPC differentiation and remyelination.

The fact that RFX4_v3 is up-regulated in MS lesions and may influence the inflammatory environment, leads to the suggestion that this factor impacts on OPC differentiation in MS.

\subsubsection{Outlook}

The present work demonstrates that compared to wild type, mice with astrocytic RFX4_v3 deletion show enhanced loss of oligodendrocytes, increased demyelination, and axonal damage. Furthermore, the process of remyelination was delayed. These observations in RFX4_v3 deficient mice were accompanied by increased microglia activation and proliferation, which can be explained by early changes in cytokine and chemokine levels as well as increased oligodendrocyte apoptosis. Further research is required to fully understand the function of the astrocytic RFX4_v3 in the cuprizone mouse model and in MS.

First, based on the literature and due to astrocytic loss of RFX4_v3 I assume that increased cytokine and chemokine levels are produced by astrocytes. However, it cannot be completely excluded that these molecules are additionally produced by microglia cells (Gandhi et al., 2010). Laser capture microdissection could be used as a method to specifically isolate astrocytes and microglia from the corpus callosum of RFX4_v3 deficient and control mice to specifically measure cytokine and chemokine transcript levels in these cells.

Second, cytokine and chemokine levels should be measured at later time points in the cuprizone mouse model to clarify whether RFX4_v3 is mainly important for early changes regarding cytokine and chemokine levels and oligodendrocyte apoptosis in this model. Such analyses could explain whether delayed remyelination is indeed a consequence of a dysregulated pro-inflammatory environment.

Third, delayed remyelination in RFX4_v3 deficient mice could be due a lack of secretion of pro-remyelinating factors by astrocytes. Limited remyelination in MS was linked to disturbed balance of growth factors that influence OPC differentiation (Webster, H. d. F, 1997; Franklin and Hinks, 1999). One of these astrocyte produced growth factors is IGF1, which has been demonstrated to influence oligodendrocyte differentiation. IGF1 was shown to be up-regulated in homozygous RFX4_v3 knockout animals at the embryonic stage 10.5 (Zhang et al., 2006a). In the work presented here up-regulation of 
IGF1 was shown in astrocytes of RFX4_v3 conditional knockout animals at the time of birth, indicating that RFX4_v3 is important for regulation of the pro-remyelinating factor IGF1. Therefore, it would be important to analyze IGF1 expression of RFX4_v3 deficient and control mice to investigate whether delayed remyelination is a consequence of dysregulated inflammatory environments and/or of a lack of secretion of proremyelinating factors by astrocytes in the cuprizone mouse model.

Fourth, members of the RFX family of transcription factors are thought to bind to the $X$-box consensus sequences in the promoters of targets to regulate gene expression. To investigate if the analyzed cytokines and chemokines are potential direct targets of the transcription factor RFX4_v3, promoter sequences should be scanned for the presence of X-box consensus sequences in silico. Furthermore, RFX4_v3 transcriptional activity on potential targets can be analyzed by reporter gene assays.

Lastly, it was demonstrated that RFX4_v3 is important for myelination and activation and recruitment of microglia by secretion of cytokines and chemokine. Based on these data and on data available in the literature it only can be speculated that RFX4_v3 may control cytokines and chemokine levels and restricts neuroinflammation in MS. Further studies are needed to understand the role of RFX4_v3 in MS, which includes a detailed and extensive immunohistochemical analysis of RFX4_v3 in different types of MS lesions. 


\section{Summary and conclusion}

\subsection{Part 1: Characterization of the GFAP cell specific RFX4 conditional knockout mice}

GFAP cell specific RFX4 conditional knockout mice were generated by crossing the RFX4 flox/flox strain with a GFAP driven Cre transgenic strain. This results in RFX4 gene inactivation by deletion of the exon 4 in vivo. In mice, the exon 4 of the RFX4 gene is shared by RFX4_v2 and RFX4_v3. Only RFX4_v3 was identified in the fetal and adult brain, while RFX4_v2 is expressed in the testis (Blackshear et al., 2003; Matsushita et al., 2005). Therefore I concluded that RFX4_v3 is deleted in GFAP cell specific RFX4 conditional knockout mice, which was further supported by the obtained brain phenotype.

A substantial proportion of the GFAP cell specific RFX4 conditional knockout mice developed severe head swelling followed by neurological deficits that led to death in young adulthood. Histological analysis of GFAP cell specific conditional knockout mice revealed a hydrocephalus with dramatic extension of the lateral ventricle. The hydrocephalus was present at birth, indicating that the hydrocephalus is congenital, which is in line with the literature (Blackshear et al., 2003).

At an age of 6 weeks the mortality rate for heterozygous RFX4_v3 conditional knockout mice was about 20\%, while homozygous RFX4_v3 conditional knockout mice died at a rate of about $50 \%$. This implies that the phenotype of RFX4_v3 conditional knockout mice is most likely the result of a gene dosage effect.

It has been reported that maldevelopment and resulting dysfunction of the SCO is involved in the genesis of aqueductal stenosis, which is the primary cause of a congenital hydrocephalus (Vio et al., 2000; Perez Figares, 2001). In transgenic RFX4_v3 +/- mice generated by Blackshear and colleagues maldevelopment and dysfunction of the SCO were described as the main cause for hydrocephalus formation (Blackshear et al., 2003). Thus I hypothesized that hydrocephalus in RFX4 conditional knockout mice is caused by maldevelopment and dysfunction of the SCO.

The SCO was present in all analyzed RFX4 conditional knockout animals. However, all symptomatic mice exhibited SCO hypoplasia. In addition, the SCO of RFX4 conditional knockout animals showed reduced or no immunoreactivity with anti-Reissner's fiber serum (AFRU), implicating a dysfunctional glycoprotein secretion of the SCO.

Furthermore, early morphogens including the Wnt signaling pathway were differentially expressed in astrocytes from RFX4 conditional knockout mice. These genes are relevant for dorsal midline structuring including the development of the SCO. These observations were in line with the findings of Blackshear and colleagues and supported the hypothesis that hydrocephalus in RFX4 conditional knockout animals is caused by maldevelopment and dysfunction of the SCO. My data further show that RFX4_v3 depletion in GFAP cell 
lineages including astrocytes and ependymal cells is sufficient to induce congenital hydrocephalus.

Altogether our results indicate that astrocytic and ependymal RFX4_v3 is highly relevant for the development of the brain and the pathogenesis of congenital hydrocephalus in rodents. An alignment of the human and mouse RFX4_v3 amino acid sequences showed $96 \%$ sequence identity between the mouse and human RFX4_v3 protein (Blackshear et al., 2003), which could point toward similar functions of RFX4_v3 in mouse and human. Therefore RFX4_v3 may be involved in the pathogenesis of human congenital hydrocephalus, which will be part of future investigations.

\subsection{Part 2: The role of astrocytic RFX4_v3 in demyelination}

The transcription factor RFX4_v3 was identified in the fetal and adult brain (Blackshear et al., 2003; Matsushita et al., 2005). In 2008, Cahoy and colleagues identified the transcription factor RFX4 as an astrocyte enriched factor (Cahoy et al., 2008).

In the present work RFX4_v3 was highly up-regulated in brain lesions of the neuroinflammatory disorder MS. IHC of active and chronic inactive MS lesions showed that RFX4 is present in various cell types and shows a prominent nuclear localization in astrocytes.

Astrocytes play an important role in modulating de- and remyelination in MS (Nair et al., 2008). They promote demyelination by enhancing the immune response through antigen presentation and secretion of chemotactic molecules (Dong and Benveniste, 2001; McFarland and Martin, 2007). Conversely, astrocytes inhibit aspects of the immune response, for example through production of anti-inflammatory cytokines, thereby inhibiting demyelination (Schönrock et al., 2000; Valerio, 2002).

To understand the astrocytic role of RFX4_v3 in MS, GFAP cell specific RFX4_v3 conditional knockout mice were used to study de- and remyelination in the cuprizone mouse model. To analyze demyelination, RFX4_v3 deficient mice and wild type mice were fed with the copper chelator cuprizone for 4 weeks. Remyelination was analyzed either 7 or 14 days after cuprizone withdrawal.

The data presented in this study demonstrate that astrocytic RFX4_v3 expression is important for regulation of myelination and activation of microglia during the process of de- and remyelination in the cuprizone mouse model. During the process of demyelination RFX4_v3 deficient mice displayed increased demyelination, reduced total numbers of oligodendrocytes and enhanced axonal damage. Remyelination in RFX4_v3 deficient mice was delayed. These effects were accompanied by increased phagocyte density in RFX4_v3 deficient mice. Phagocyte density correlated well with the course of de- and remyelination in RFX4_v3 deficient mice. 
The results raised two central questions. i) What is the reason for the massive phagocyte accumulation? and ii) is phagocyte accumulation the cause or consequence of increased demyelination and delayed remyelination in RFX4_v3 deficient mice?

To answer these questions the origin of the Mac3-positive cells in the corpus callosum was investigated. In this study it was shown that the BBB in RFX4_v3 deficient mice is closed, which is in line with previous studies (Kondo et al., 1987; Baker et al., 1991; McMahon et al., 2002). This implicates that the Mac3-positive cells in RFX4_v3 deficient mice represent microglia and not blood-borne macrophages, which could enter via a disrupted BBB.

Flow cytometry analysis showed that the CD45-low and CD11b-positive cell population that identifies parenchymal microglia cells, is the most abundant cell population in RFX4_v3 deficient mice, which was in line with previous studies (Remington et al., 2007; Voss et al., 2012). This population increased in absolute numbers after 4 weeks of cuprizone treatment without changing the expression of activation markers on microglia cells during demyelination. These results indicated that loss of RFX4_v3 in the astrocytes leads to increased microglia recruitment and proliferation in the corpus callosum in mice exposed to cuprizone, but does not significantly alter the expression of activation markers on microglia cells during demyelination.

Further investigations during the early phase of cuprizone treatment showed increased transcript levels of pro-inflammatory chemokines and cytokines and enhanced oligodendrocyte apoptosis in the corpus callosum of RFX4_v3 deficient mice. Based on the literature most of these pro-inflammatory factors are produced by astrocytes and play an important role for leukocyte activation and recruitment in the cuprizone mouse model, EAE as well as in MS lesions. This implied that the transcription factor RFX4_v3 controls the activation and response of astrocytes by producing chemokines and cytokines and thereby influences the recruitment of phagocytes. However, in this study the observed microglia phenotype in the cuprizone mouse model of RFX4_v3 deficient mice seems to be a combination of both enhanced cytokine and chemokine secretion and increased oligodendrocyte apoptosis, which is in line with the literature (Li et al., 2008; Raasch et al., 2011; Skripuletz et al., 2013). In this work it cannot be completely solved whether enhanced microglia recruitment and proliferation is the cause or consequence of the increased demyelination, reduced oligodendrocytes and increased axonal damage in the corpus callosum of RFX4_v3 deficient mice. 
Altogether these results indicate that RFX4_v3 in astrocytes plays a protective role in the cuprizone mouse model by regulating oligodendrocyte apoptosis and recruitment of microglia cells. Our data point towards a control of the inflammatory environment by the transcription factor RFX4_v3 to restrict the activation and recruitment of microglia cells as well as oligodendrocyte apoptosis, which eventually controls the extent of de- and remyelination in the cuprizone mouse model.

Up-regulated levels of RFX4_v3 in astrocytes in MS lesions may implicate a protective role for RFX4_v3 in MS since the transcription factor was rather described as transcriptional repressor than as transcriptional activator. The transcription factor RFX4_v3 possibly acts as a regulator for the production and release of cytokines and chemokines and may have a protective role in MS by restricting neuroinflammation. 


\section{References}

Aguzzi, A., Barres, B.A., and Bennett, M.L. (2013). Microglia: scapegoat, saboteur, or something else? Science (New York, N.Y.) 339, 156-161.

Ajami, B., Bennett, J.L., Krieger, C., Tetzlaff, W., and Rossi, Fabio M V (2007). Local self-renewal can sustain CNS microglia maintenance and function throughout adult life. Nature neuroscience 10 , 1538-1543.

Araki, R., Takahashi, H., Fukumura, R., Sun, F., Umeda, N., Sujino, M., Inouye, S.-I.T., Saito, T., and Abe, M. (2004). Restricted expression and photic induction of a novel mouse regulatory factor X4 transcript in the suprachiasmatic nucleus. The Journal of biological chemistry 279, 10237-10242.

Arnett, H.A., Mason, J., Marino, M., Suzuki, K., Matsushima, G.K., and Ting, J.P. (2001). TNF alpha promotes proliferation of oligodendrocyte progenitors and remyelination. Nature neuroscience 4 , 1116-1122.

Ashique, A.M., Choe, Y., Karlen, M., May, S.R., Phamluong, K., Solloway, M.J., Ericson, J., and Peterson, A.S. (2009). The Rfx4 transcription factor modulates Shh signaling by regional control of ciliogenesis. Science signaling 2, ra70.

Baas, D., Meiniel, A., Benadiba, C., Bonnafe, E., Meiniel, O., Reith, W., and Durand, B. (2006). A deficiency in RFX3 causes hydrocephalus associated with abnormal differentiation of ependymal cells. The European journal of neuroscience 24, 1020-1030.

Babcock, A.A., Kuziel, W.A., Rivest, S., and Owens, T. (2003). Chemokine expression by glial cells directs leukocytes to sites of axonal injury in the CNS. The Journal of neuroscience : the official journal of the Society for Neuroscience 23, 7922-7930.

Bach, A. (2003). Msx1 is required for dorsal diencephalon patterning. Development 130, 4025-4036.

Baker, D., O'Neill, J.K., and Turk, J.L. (1991). Cytokines in the central nervous system of mice during chronic relapsing experimental allergic encephalomyelitis. Cellular Immunology 134, 505-510.

Bakker, D.A., and Ludwin, S.K. (1987). Blood-brain barrier permeability during Cuprizone-induced demyelination. Journal of the Neurological Sciences 78, 125-137. 
Ballabh, P., Braun, A., and Nedergaard, M. (2004). The blood-brain barrier: an overview: structure, regulation, and clinical implications. Neurobiology of disease 16, 1-13.

Barnett, M.H., and Prineas, J.W. (2004). Relapsing and remitting multiple sclerosis: pathology of the newly forming lesion. Annals of neurology 55, 458-468.

Barnett, M.H., and Sutton, I. (2006). The pathology of multiple sclerosis: a paradigm shift. Current opinion in neurology 19, 242-247.

Barres, B.A., Schmid, R., Sendnter, M., and Raff, M.C. (1993). Multiple extracellular signals are required for long-term oligodendrocyte survival. Development (Cambridge, England) 118, 283-295.

Bauer, J., Sminia, T., Wouterlood, F.G., and Dijkstra, C.D. (1994). Phagocytic activity of macrophages and microglial cells during the course of acute and chronic relapsing experimental autoimmune encephalomyelitis. Journal of neuroscience research 38, 365-375.

Beck, K.D., Powell-Braxtont, L., Widmer, H.-R., Valverde, J., and Hefti, F. (1995). Igf1 gene disruption results in reduced brain size, CNS hypomyelination, and loss of hippocampal granule and striatal parvalbumin-containing neurons. Neuron 14, 717-730.

Benadiba, C., Magnani, D., Niquille, M., Morlé, L., Valloton, D., Nawabi, H., Ait-Lounis, A., Otsmane, B., Reith, W., and Theil, T., et al. (2012). The ciliogenic transcription factor RFX3 regulates early midline distribution of guidepost neurons required for corpus callosum development. PLoS genetics 8, e1002606.

Bénardais, K., Kotsiari, A., Skuljec, J., Koutsoudaki, P.N., Gudi, V., Singh, V., Vulinović, F., Skripuletz, T., and Stangel, M. (2013). Cuprizone [bis(cyclohexylidenehydrazide)] is selectively toxic for mature oligodendrocytes. Neurotoxicity research 24, 244-250.

Bennett, L., Yang, M., Enikolopov, G., and lacovitti, L. (2009). Circumventricular organs: a novel site of neural stem cells in the adult brain. Molecular and cellular neurosciences 41, 337-347.

Benveniste, E.N. (1997). Role of macrophages/microglia in multiple sclerosis and experimental allergic encephalomyelitis. Journal of molecular medicine (Berlin, Germany) 75, 165-173.

Bianchi, R., Kastrisianaki, E., Giambanco, I., and Donato, R. (2011). S100B protein stimulates microglia migration via RAGE-dependent up-regulation of chemokine expression and release. The Journal of biological chemistry 286, 7214-7226. 
Bjartmar, C., and Trapp, B.D. (2001). Axonal and neuronal degeneration in multiple sclerosis: mechanisms and functional consequences. Current opinion in neurology 14, 271-278.

Blackshear, P.J., Graves, J.P., Stumpo, D.J., Cobos, I., Rubenstein, John L R, and Zeldin, D.C. (2003). Graded phenotypic response to partial and complete deficiency of a brain-specific transcript variant of the winged helix transcription factor RFX4. Development (Cambridge, England) 130, 4539-4552.

Blauth, K., Zhang, X., Chopra, M., Rogan, S., and Markovic-Plese, S. (2015). The role of fractalkine (CX3CL1) in regulation of $\mathrm{CD} 4(+)$ cell migration to the central nervous system in patients with relapsing-remitting multiple sclerosis. Clinical immunology (Orlando, Fla.).

Boillat, C.A., Jones, H.C., and Kaiser, G.L. (1999). Aqueduct stenosis in hydrocephalus: ultrastructural investigation in neonatal H-Tx rat brain. European journal of pediatric surgery : official journal of Austrian Association of Pediatric Surgery ... [et al] = Zeitschrift für Kinderchirurgie 9 Suppl 1, 44-46.

Bradley, J.R. (2008). TNF-mediated inflammatory disease. The Journal of pathology 214, 149-160.

Brosnan, C.F., and Raine, C.S. (2013). The astrocyte in multiple sclerosis revisited. Glia 61, 453-465.

Broux, B., Pannemans, K., Zhang, X., Markovic-Plese, S., Broekmans, T., Eijnde, B.O., van Wijmeersch, B., Somers, V., Geusens, P., and van der Pol, Susanne, et al. (2012). CX(3)CR1 drives cytotoxic CD4(+)CD28(-) T cells into the brain of multiple sclerosis patients. Journal of autoimmunity 38, 10-19.

Brück, W. (2005). Clinical implications of neuropathological findings in multiple sclerosis. Journal of neurology 252 Suppl 3, iii10-iii14.

Brück, W., Pförtner, R., Pham, T., Zhang, J., Hayardeny, L., Piryatinsky, V., Hanisch, U.-K., Regen, T., van Rossum, D., and Brakelmann, L., et al. (2012). Reduced astrocytic NF-KB activation by laquinimod protects from cuprizone-induced demyelination. Acta neuropathologica 124, 411-424.

Brück, W., Porada, P., Poser, S., Rieckmann, P., Hanefeld, F., Kretzschmar, H.A., and Lassmann, H. (1995). Monocyte/macrophage differentiation in early multiple sclerosis lesions. Annals of neurology $38,788-796$.

Brück, W., and Stadelmann, C. (2005). The spectrum of multiple sclerosis: new lessons from pathology. Current opinion in neurology 18, 221-224.

Bruni, J.E., Del Bigio, M.R., Cardoso, E.R., and Persaud, T. (1988a). Hereditary hydrocephalus in laboratory animals and humans. Experimental pathology 35, 239-246. 
Bruni, J.E., del Bigio, M R, Cardoso, E.R., and Persaud, T.V. (1988b). Neuropathology of congenital hydrocephalus in the SUMS/NP mouse. Acta neurochirurgica 92, 118-122.

Bsibsi, M., Persoon-Deen, C., Verwer, Ronald W H, Meeuwsen, S., Ravid, R., and Van Noort, Johannes M (2006). Toll-like receptor 3 on adult human astrocytes triggers production of neuroprotective mediators. Glia 53, 688-695.

Bsibsi, M., Ravid, R., Gveric, D., and Van Noort, Johannes M (2002). Broad expression of Toll-like receptors in the human central nervous system. Journal of neuropathology and experimental neurology 61, 1013-1021.

Buschmann, J.P., Berger, K., Awad, H., Clarner, T., Beyer, C., and Kipp, M. (2012). Inflammatory response and chemokine expression in the white matter corpus callosum and gray matter cortex region during cuprizone-induced demyelination. Journal of molecular neuroscience : MN 48, 66-76.

Bush, T.G., Puvanachandra, N., Horner, C.H., Polito, A., Ostenfeld, T., Svendsen, C.N., Mucke, L., Johnson, M.H., and Sofroniew, M.V. (1999). Leukocyte infiltration, neuronal degeneration, and neurite outgrowth after ablation of scar-forming, reactive astrocytes in adult transgenic mice. Neuron 23, 297-308.

Cahoy, J.D., Emery, B., Kaushal, A., Foo, L.C., Zamanian, J.L., Christopherson, K.S., Xing, Y., Lubischer, J.L., Krieg, P.A., and Krupenko, S.A., et al. (2008). A transcriptome database for astrocytes, neurons, and oligodendrocytes: a new resource for understanding brain development and function. The Journal of neuroscience : the official journal of the Society for Neuroscience 28, 264-278.

Calderon, T.M., Eugenin, E.A., Lopez, L., Kumar, S.S., Hesselgesser, J., Raine, C.S., and Berman, J.W. (2006). A role for CXCL12 (SDF-1alpha) in the pathogenesis of multiple sclerosis: regulation of CXCL12 expression in astrocytes by soluble myelin basic protein. Journal of Neuroimmunology 177, 27-39.

Cannella, B., and Raine, C.S. (1995). The adhesion molecule and cytokine profile of multiple sclerosis lesions. Annals of neurology 37, 424-435.

Carpentier, P.A., Williams, B.R., and Miller, S.D. (2007). Distinct roles of protein kinase R and toll-like receptor 3 in the activation of astrocytes by viral stimuli. Glia 55, 239-252.

Castañeyra-Perdomo, A., Meyer, G., Carmona-Calero, E., Bañuelos-Pineda, J., Méndez-Medina, R., Ormazabal-Ramos, C., and Ferres-Torres, R. (1994). Alterations of the subcommissural organ in the hydrocephalic human fetal brain. Brain research. Developmental brain research 79, 316-320. 
Chouaf, L., Didier-Bazes, M., Hardin, H., Aguera, M., Fevre-Montange, M., Voutsinos, B., and Belin, M.F. (1991). Developmental expression of glial markers in ependymocytes of the rat subcommissural organ: role of the environment. Cell Tissue Res 266, 553-561.

Clevers, H., and Nusse, R. (2012). Wnt/ $\beta$-catenin signaling and disease. Cell 149, 1192-1205.

Compston, A., and Coles, A. (2008). Multiple sclerosis. The Lancet 372, 1502-1517.

Cross, A.K., and Woodroofe, M.N. (2001). Immunoregulation of microglial functional properties. Microscopy research and technique 54, 10-17.

Croxford, A.L., Kurschus, F.C., and Waisman, A. (2011). Mouse models for multiple sclerosis: historical facts and future implications. Biochimica et biophysica acta 1812, 177-183.

Cua, D.J., Sherlock, J., Chen, Y., Murphy, C.A., Joyce, B., Seymour, B., Lucian, L., To, W., Kwan, S., and Churakova, T., et al. (2003). Interleukin-23 rather than interleukin-12 is the critical cytokine for autoimmune inflammation of the brain. Nature $421,744-748$.

Cudrici, C., Niculescu, T., Niculescu, F., Shin, M.L., and Rus, H. (2006). Oligodendrocyte cell death in pathogenesis of multiple sclerosis: Protection of oligodendrocytes from apoptosis by complement. Journal of rehabilitation research and development 43, 123-132.

de Lau, Wim B M, Snel, B., and Clevers, H.C. (2012). The R-spondin protein family. Genome biology $13,242$.

Didier, N., Romero, I.A., Créminon, C., Wijkhuisen, A., Grassi, J., and Mabondzo, A. (2003). Secretion of interleukin-1 beta by astrocytes mediates endothelin-1 and tumour necrosis factor-alpha effects on human brain microvascular endothelial cell permeability. Journal of neurochemistry 86, 246-254.

Didon, L., Zwick, R.K., Chao, I.W., Walters, M.S., Wang, R., Hackett, N.R., and Crystal, R.G. (2013). RFX3 modulation of FOXJ1 regulation of cilia genes in the human airway epithelium. Respiratory research 14,70 .

Dietrich, P., Shanmugasundaram, R., Shuyu, E., and Dragatsis, I. (2009). Congenital hydrocephalus associated with abnormal subcommissural organ in mice lacking huntingtin in Wnt1 cell lineages. Human molecular genetics 18, 142-150.

Dong, Y., and Benveniste, E.N. (2001). Immune function of astrocytes. Glia 36, 180-190. 
Dotzlaw, H., Alkhalaf, M., and Murphy, L.C. (1992). Characterization of estrogen receptor variant mRNAs from human breast cancers. Molecular endocrinology (Baltimore, Md.) 6, 773-785.

D'Souza, S.D. (1996). Multiple Sclerosis: Fas Signaling in Oligodendrocyte Cell Death. Journal of Experimental Medicine 184, 2361-2370.

El Zein, L., Ait-Lounis, A., Morlé, L., Thomas, J., Chhin, B., Spassky, N., Reith, W., and Durand, B. (2009). RFX3 governs growth and beating efficiency of motile cilia in mouse and controls the expression of genes involved in human ciliopathies. Journal of Cell Science 122, 3180-3189.

Emery, P., Durand, B., Mach, B., and Reith, W. (1996). RFX Proteins, a Novel Family of DNA Binding Proteins Conserved in the Eukaryotic Kingdom. Nucleic Acids Research 24, 803-807.

Eng, L.F. (1985). Glial fibrillary acidic protein (GFAP): the major protein of glial intermediate filaments in differentiated astrocytes. Journal of Neuroimmunology 8, 203-214.

Estivill-Torrús, G., Vitalis, T., Fernández-Llebrez, P., and Price, D.J. (2001). The transcription factor Pax6 is required for development of the diencephalic dorsal midline secretory radial glia that form the subcommissural organ. Mechanisms of development 109, 215-224.

Feng, C., Zhang, Y., Yin, J., Li, J., Abounader, R., and Zuo, Z. (2014). Regulatory factor X1 is a new tumor suppressive transcription factor that acts via direct downregulation of CD44 in glioblastoma. Neuro-oncology 16, 1078-1085.

Fernández-Llebrez, P., Grondona, J.M., Pérez, J., López-Aranda, M.F., Estivill-Torrús, G., Llebrez-Zayas, P.F., Soriano, E., Ramos, C., Lallemand, Y., and Bach, A., et al. (2004). Msx1-deficient mice fail to form prosomere 1 derivatives, subcommissural organ, and posterior commissure and develop hydrocephalus. Journal of neuropathology and experimental neurology 63, 574-586.

Franklin, R.J., and Hinks, G.L. (1999). Understanding CNS remyelination: clues from developmental and regeneration biology. Journal of neuroscience research 58, 207-213.

Franklin, Robin J M (2002). Why does remyelination fail in multiple sclerosis? Nature reviews. Neuroscience 3, 705-714.

Franklin, Robin J M, and ffrench-Constant, C. (2008). Remyelination in the CNS: from biology to therapy. Nature reviews. Neuroscience 9, 839-855. 
Furuta, Y., Piston, D.W., and Hogan, B.L. (1997). Bone morphogenetic proteins (BMPs) as regulators of dorsal forebrain development. Development (Cambridge, England) 124, 2203-2212.

Gajiwala, K.S., Chen, H., Cornille, F., Roques, B.P., Reith, W., Mach, B., and Burley, S.K. (2000). Structure of the winged-helix protein hRFX1 reveals a new mode of DNA binding. Nature $403,916-$ 921.

Gandhi, R., Laroni, A., and Weiner, H.L. (2010). Role of the innate immune system in the pathogenesis of multiple sclerosis. Journal of Neuroimmunology 221, 7-14.

Gangemi, M., Cavallo, L.M., Di Somma, A., Mazzucco, G.M., Bono, P.S., Ghetti, G., and Zambon, G. (2014). Hydrocephalus onset after microsurgical or endovascular treatment for acute subarachnoid hemorrhage. Retrospective italian multicenter study. Translational medicine @ UniSa 9, 50-55.

Garcia, A Denise R, Doan, N.B., Imura, T., Bush, T.G., and Sofroniew, M.V. (2004). GFAP-expressing progenitors are the principal source of constitutive neurogenesis in adult mouse forebrain. Nature neuroscience $7,1233-1241$.

Gensert, J.M., and Goldman, J.E. (1997). Endogenous Progenitors Remyelinate Demyelinated Axons in the Adult CNS. Neuron 19, 197-203.

Gimenez, Mary Ann T, Sim, J.E., and Russell, J.H. (2004). TNFR1-dependent VCAM-1 expression by astrocytes exposes the CNS to destructive inflammation. Journal of Neuroimmunology 151, 116-125.

Giulian, D., and Lachman, L. (1985). Interleukin-1 stimulation of astroglial proliferation after brain injury. Science 228, 497-499.

Goldman, S. (2003). Glia as neural progenitor cells. Trends in neurosciences 26, 590-596.

Grondona, J.M., Pérez, J., Cifuentes, M., López-Avalos, M.D., Nualart, F.J., Peruzzo, B., FernándezLlebrez, P., and Rodríguez, E.M. (1994). Analysis of the secretory glycoproteins of the subcommissural organ of the dogfish (Scyliorhinus canicula). Molecular Brain Research 26, 299-308.

Gudi, V., Gingele, S., Skripuletz, T., and Stangel, M. (2014). Glial response during cuprizone-induced de- and remyelination in the CNS: lessons learned. Frontiers in cellular neuroscience 8, 73.

Gudi, V., Moharregh-Khiabani, D., Skripuletz, T., Koutsoudaki, P.N., Kotsiari, A., Skuljec, J., Trebst, C., and Stangel, M. (2009). Regional differences between grey and white matter in cuprizone induced demyelination. Brain Research 1283, 127-138. 
Hayashi, K., Nakamura, S., Nishida, W., and Sobue, K. (2006). Bone morphogenetic protein-induced MSX1 and MSX2 inhibit myocardin-dependent smooth muscle gene transcription. Molecular and cellular biology 26, 9456-9470.

Hayashi, M., Luo, Y., Laning, J., Strieter, R.M., and Dorf, M.E. (1995). Production and function of monocyte chemoattractant protein-1 and other $\beta$-chemokines in murine glial cells. Journal of Neuroimmunology 60, 143-150.

Hébert, J.M., Mishina, Y., and McConnell, S.K. (2002). BMP signaling is required locally to pattern the dorsal telencephalic midline. Neuron 35, 1029-1041.

Herx, L.M., and Yong, V.W. (2001). Interleukin-1 beta is required for the early evolution of reactive astrogliosis following CNS lesion. Journal of neuropathology and experimental neurology 60, 961971.

Hesse, A., Wagner, M., Held, J., Brück, W., Salinas-Riester, G., Hao, Z., Waisman, A., and Kuhlmann, T. (2010). In toxic demyelination oligodendroglial cell death occurs early and is FAS independent. Neurobiology of disease 37, 362-369.

Hiremath, M.M., Saito, Y., Knapp, G.W., Ting, J.-Y., Suzuki, K., and Matsushima, G.K. (1998). Microglial/macrophage accumulation during cuprizone-induced demyelination in C57BL/6 mice. Journal of Neuroimmunology 92, 38-49.

Hisahara, S., Shoji, S., Okano, H., and Miura, M. (1997). ICE/CED-3 Family Executes Oligodendrocyte Apoptosis by Tumor Necrosis Factor. Journal of neurochemistry 69, 10-20.

Huh, M.S., Todd, Matthew A M, and Picketts, D.J. (2009). SCO-ping out the mechanisms underlying the etiology of hydrocephalus. Physiology (Bethesda, Md.) 24, 117-126.

Jack, C.S., Arbour, N., Manusow, J., Montgrain, V., Blain, M., McCrea, E., Shapiro, A., and Antel, J.P. (2005). TLR signaling tailors innate immune responses in human microglia and astrocytes. Journal of immunology (Baltimore, Md. : 1950) 175, 4320-4330.

Jones, H.C., Dack, S., and Ellis, C. (1987). Morphological aspects of the development of hydrocephalus in a mouse mutant (SUMS/NP). Acta neuropathologica 72, 268-276.

Jones, H.C., and Klinge, P.M. (2008). Hydrocephalus 2008, 17-20th September, Hannover Germany: a conference report. Cerebrospinal fluid research 5, 19. 
Kang, J., Jiang, L., Goldman, S.A., and Nedergaard, M. (1998). Astrocyte-mediated potentiation of inhibitory synaptic transmission. Nature neuroscience 1, 683-692.

Karni, A., Abraham, M., Monsonego, A., Cai, G., Freeman, G.J., Hafler, D., Khoury, S.J., and Weiner, H.L. (2006). Innate Immunity in Multiple Sclerosis: Myeloid Dendritic Cells in Secondary Progressive Multiple Sclerosis Are Activated and Drive a Proinflammatory Immune Response. The Journal of Immunology 177, 4196-4202.

Karpus, W.J., Lukacs, N.W., McRae, B.L., Strieter, R.M., Kunkel, S.L., and Miller, S.D. (1995). An important role for the chemokine macrophage inflammatory protein-1 alpha in the pathogenesis of the $T$ cell-mediated autoimmune disease, experimental autoimmune encephalomyelitis. Journal of immunology (Baltimore, Md. : 1950) 155, 5003-5010.

Kennedy, K.J., Strieter, R.M., Kunkel, S.L., Lukacs, N.W., and Karpus, W.J. (1998). Acute and relapsing experimental autoimmune encephalomyelitis are regulated by differential expression of the CC chemokines macrophage inflammatory protein-1 $\alpha$ and monocyte chemotactic protein-1. Journal of Neuroimmunology 92, 98-108.

Kerschensteiner, M., Stadelmann, C., Dechant, G., Wekerle, H., and Hohlfeld, R. (2003). Neurotrophic cross-talk between the nervous and immune systems: implications for neurological diseases. Annals of neurology 53, 292-304.

Kettenmann, H., Hanisch, U.-K., Noda, M., and Verkhratsky, A. (2011). Physiology of microglia. Physiological reviews 91, 461-553.

Kierdorf, K., Erny, D., Goldmann, T., Sander, V., Schulz, C., Perdiguero, E.G., Wieghofer, P., Heinrich, A., Riemke, P., and Hölscher, C., et al. (2013). Microglia emerge from erythromyeloid precursors via Pu.1- and Irf8-dependent pathways. Nature neuroscience 16, 273-280.

Kim, R.Y., Hoffman, A.S., Itoh, N., Ao, Y., Spence, R., Sofroniew, M.V., and Voskuhl, R.R. (2014). Astrocyte CCL2 sustains immune cell infiltration in chronic experimental autoimmune encephalomyelitis. Journal of Neuroimmunology 274, 53-61.

Komiya, Y., and Habas, R. (2014). Wnt signal transduction pathways. Organogenesis 4, 68-75.

Kondo, A., Nakano, T., and Suzuki, K. (1987). Blood-brain barrier permeability to horseradish peroxidase in twitcher and cuprizone-intoxicated mice. Brain Research 425, 186-190. 
Kotter, M.R., Li, W.-W., Zhao, C., and Franklin, Robin J M (2006). Myelin impairs CNS remyelination by inhibiting oligodendrocyte precursor cell differentiation. The Journal of neuroscience : the official journal of the Society for Neuroscience 26, 328-332.

Koutsoudaki, P.N., Hildebrandt, H., Gudi, V., Skripuletz, T., Škuljec, J., and Stangel, M. (2010). Remyelination after cuprizone induced demyelination is accelerated in mice deficient in the polysialic acid synthesizing enzyme St8sialV. Neuroscience 171, 235-244.

Koutsoudaki, P.N., Skripuletz, T., Gudi, V., Moharregh-Khiabani, D., Hildebrandt, H., Trebst, C., and Stangel, M. (2009). Demyelination of the hippocampus is prominent in the cuprizone model. Neuroscience letters 451, 83-88.

Krauthausen, M., Saxe, S., Zimmermann, J., Emrich, M., Heneka, M.T., and Müller, M. (2014). CXCR3 modulates glial accumulation and activation in cuprizone-induced demyelination of the central nervous system. Journal of neuroinflammation 11, 109.

Kutzelnigg, A., and Lassmann, H. (2014). Pathology of multiple sclerosis and related inflammatory demyelinating diseases. Handbook of clinical neurology 122, 15-58.

Kwon, T., Chung, M.-I., Gupta, R., Baker, J.C., Wallingford, J.B., and Marcotte, E.M. (2014). Identifying direct targets of transcription factor Rfx2 that coordinate ciliogenesis and cell movement. Genomics data 2, 192-194.

LaMantia, A.S., Colbert, M.C., and Linney, E. (1993). Retinoic acid induction and regional differentiation prefigure olfactory pathway formation in the mammalian forebrain. Neuron 10, 10351048.

Langrish, C.L., Chen, Y., Blumenschein, W.M., Mattson, J., Basham, B., Sedgwick, J.D., McClanahan, T., Kastelein, R.A., and Cua, D.J. (2005). IL-23 drives a pathogenic T cell population that induces autoimmune inflammation. The Journal of experimental medicine 201, 233-240.

Lassmann, H. (2011). Review: the architecture of inflammatory demyelinating lesions: implications for studies on pathogenesis. Neuropathology and applied neurobiology 37, 698-710.

Lassmann, H., Brück, W., and Lucchinetti, C.F. (2007). The immunopathology of multiple sclerosis: an overview. Brain pathology (Zurich, Switzerland) 17, 210-218. 
Lee, S.C., Dickson, D.W., Liu, W., and Brosnan, C.F. (1993). Induction of nitric oxide synthase activity in human astrocytes by interleukin-1 beta and interferon-gamma. Journal of Neuroimmunology 46, $19-24$.

Lee, S.M., Tole, S., Grove, E., and McMahon, A.P. (2000). A local Wnt-3a signal is required for development of the mammalian hippocampus. Development (Cambridge, England) 127, 457-467.

Li, J., Ghiani, C.A., Kim, J.Y., Liu, A., Sandoval, J., DeVellis, J., and Casaccia-Bonnefil, P. (2008). Inhibition of p53 transcriptional activity: a potential target for future development of therapeutic strategies for primary demyelination. The Journal of neuroscience : the official journal of the Society for Neuroscience 28, 6118-6127.

Liberto, C.M., Albrecht, P.J., Herx, L.M., Yong, V.W., and Levison, S.W. (2004). Pro-regenerative properties of cytokine-activated astrocytes. Journal of neurochemistry 89, 1092-1100.

Liem, K.F., Tremml, G., and Jessell, T.M. (1997). A Role for the Roof Plate and Its Resident TGFßRelated Proteins in Neuronal Patterning in the Dorsal Spinal Cord. Cell 91, 127-138.

Liem, K.F., Tremml, G., Roelink, H., and Jessell, T.M. (1995). Dorsal differentiation of neural plate cells induced by BMP-mediated signals from epidermal ectoderm. Cell 82, 969-979.

Lindner, M., Heine, S., Haastert, K., Garde, N., Fokuhl, J., Linsmeier, F., Grothe, C., Baumgärtner, W., and Stangel, M. (2008). Sequential myelin protein expression during remyelination reveals fast and efficient repair after central nervous system demyelination. Neuropathology and applied neurobiology 34, 105-114.

Linninger, A.A., Sweetman, B., and Penn, R. (2009). Normal and hydrocephalic brain dynamics: the role of reduced cerebrospinal fluid reabsorption in ventricular enlargement. Annals of biomedical engineering $37,1434-1447$.

Liu, A., and Niswander, L.A. (2005). Bone morphogenetic protein signalling and vertebrate nervous system development. Nature reviews. Neuroscience 6, 945-954.

Liu, Y., Helms, A.W., and Johnson, J.E. (2004). Distinct activities of Msx1 and Msx3 in dorsal neural tube development. Development (Cambridge, England) 131, 1017-1028. 
Locatelli, G., Wörtge, S., Buch, T., Ingold, B., Frommer, F., Sobottka, B., Krüger, M., Karram, K., Bühlmann, C., and Bechmann, I., et al. (2012). Primary oligodendrocyte death does not elicit anti-CNS immunity. Nature neuroscience 15, 543-550.

Louis, J., Magal, E., Takayama, S., and Varon, S. (1993). CNTF protection of oligodendrocytes against natural and tumor necrosis factor-induced death. Science 259, 689-692.

Louvi A and Wassef M (2000). Ectopic engrailed 1 expression in the dorsal midline causes cell de... PubMed - NCBI. http://www.ncbi.nlm.nih.gov/pubmed/10952903. 26.11.2014.

Lublin, F.D., Reingold, S.C., Cohen, J.A., Cutter, G.R., Sørensen, P.S., Thompson, A.J., Wolinsky, J.S., Balcer, L.J., Banwell, B., and Barkhof, F., et al. (2014). Defining the clinical course of multiple sclerosis: the 2013 revisions. Neurology 83, 278-286.

Lucchinetti, C., Brück, W., and Noseworthy, J. (2001). Multiple sclerosis: recent developments in neuropathology, pathogenesis, magnetic resonance imaging studies and treatment. Current opinion in neurology 14, 259-269.

Lucchinetti, C., Brück, W., Parisi, J., Scheithauer, B., Rodriguez, M., and Lassmann, H. (2000). Heterogeneity of multiple sclerosis lesions: implications for the pathogenesis of demyelination. Annals of neurology $47,707-717$.

Lucchinetti, C.F., Brueck, W., Rodriguez, M., and Lassmann, H. (1998). Multiple sclerosis: lessons from neuropathology. Seminars in neurology 18, 337-349.

Mahad, D.J., and Ransohoff, R.M. (2003). The role of MCP-1 (CCL2) and CCR2 in multiple sclerosis and experimental autoimmune encephalomyelitis (EAE). Seminars in Immunology 15, 23-32.

Mason, J.L., Jones, J.J., Taniike, M., Morell, P., Suzuki, K., and Matsushima, G.K. (2000). Mature oligodendrocyte apoptosis precedes IGF-1 production and oligodendrocyte progenitor accumulation and differentiation during demyelination/remyelination. Journal of neuroscience research 61,251 262.

Mason, J.L., Langaman, C., Morell, P., Suzuki, K., and Matsushima, G.K. (2001a). Episodic demyelination and subsequent remyelination within the murine central nervous system: changes in axonal calibre. Neuropathol Appl Neurobiol 27, 50-58. 
Mason, J.L., Suzuki, K., Chaplin, D.D., and Matsushima, G.K. (2001b). Interleukin-1beta promotes repair of the CNS. The Journal of neuroscience : the official journal of the Society for Neuroscience $21,7046-7052$.

Mason, J.L., Toews, A., Hostettler, J.D., Morell, P., Suzuki, K., Goldman, J.E., and Matsushima, G.K. (2004). Oligodendrocytes and Progenitors Become Progressively Depleted within Chronically Demyelinated Lesions. The American journal of pathology 164, 1673-1682.

Matsushima, G.K., and Morell, P. (2001). The Neurotoxicant, Cuprizone, as a Model to Study Demyelination and Remyelination in the Central Nervous System. Brain Pathology 11, 107-116.

Matsushita, H., Uenaka, A., Ono, T., Hasegawa, K., Sato, S., Koizumi, F., Nakagawa, K., Toda, M., Shingo, T., and Ichikawa, T., et al. (2005). Identification of glioma-specific RFX4-E and -F isoforms and humoral immune response in patients. Cancer science 96, 801-809.

Mayo, L., Trauger, S.A., Blain, M., Nadeau, M., Patel, B., Alvarez, J.I., Mascanfroni, I.D., Yeste, A., Kivisäkk, P., and Kallas, K., et al. (2014). Regulation of astrocyte activation by glycolipids drives chronic CNS inflammation. Nature medicine 20, 1147-1156.

McDonald, W.I., Compston, A., Edan, G., Goodkin, D., Hartung, H.P., Lublin, F.D., McFarland, H.F., Paty, D.W., Polman, C.H., and Reingold, S.C., et al. (2001). Recommended diagnostic criteria for multiple sclerosis: guidelines from the International Panel on the diagnosis of multiple sclerosis. Annals of neurology 50, 121-127.

McFarland, H.F., and Martin, R. (2007). Multiple sclerosis: a complicated picture of autoimmunity. Nature immunology 8, 913-919.

McMahon, E.J., Cook, D.N., Suzuki, K., and Matsushima, G.K. (2001). Absence of MacrophageInflammatory Protein-1 Delays Central Nervous System Demyelination in the Presence of an Intact Blood-Brain Barrier. The Journal of Immunology 167, 2964-2971.

McMahon, E.J., Suzuki, K., and Matsushima, G.K. (2002). Peripheral macrophage recruitment in cuprizone-induced CNS demyelination despite an intact blood-brain barrier. Journal of Neuroimmunology 130, 32-45.

Merrill, J.E., Ignarro, L.J., Sherman, M.P., Melinek, J., and Lane, T.E. (1993). Microglial cell cytotoxicity of oligodendrocytes is mediated through nitric oxide. Journal of immunology (Baltimore, Md. : 1950) $151,2132-2141$. 
Mildner, A., Schmidt, H., Nitsche, M., Merkler, D., Hanisch, U.-K., Mack, M., Heikenwalder, M., Brück, W., Priller, J., and Prinz, M. (2007). Microglia in the adult brain arise from Ly-6ChiCCR2+ monocytes only under defined host conditions. Nature neuroscience 10, 1544-1553.

Miljkovic, D., Momcilovic, M., Stojanovic, I., Stosic-Grujicic, S., Ramic, Z., and Mostarica-Stojkovic, M. (2007). Astrocytes stimulate interleukin-17 and interferon-gamma production in vitro. Journal of neuroscience research 85, 3598-3606.

Mills Ko, E., Ma, J.H., Guo, F., Miers, L., Lee, E., Bannerman, P., Burns, T., Ko, D., Sohn, J., and Soulika, A.M., et al. (2014). Deletion of astroglial CXCL10 delays clinical onset but does not affect progressive axon loss in a murine autoimmune multiple sclerosis model. Journal of neuroinflammation 11, 105.

Milo, R., and Kahana, E. (2010). Multiple sclerosis: geoepidemiology, genetics and the environment. Autoimmunity reviews 9, A387-94.

Minagar, A., and Alexander, J.S. (2003). Blood-brain barrier disruption in multiple sclerosis. Multiple sclerosis (Houndmills, Basingstoke, England) 9, 540-549.

Miranda-Angulo, A.L., Byerly, M.S., Mesa, J., Wang, H., and Blackshaw, S. (2014). Rax regulates hypothalamic tanycyte differentiation and barrier function in mice. The Journal of comparative neurology 522, 876-899.

Moreno, C.S., Rogers, E.M., Brown, J.A., and Boss, J.M. (1997). Regulatory factor X, a bare lymphocyte syndrome transcription factor, is a multimeric phosphoprotein complex. Journal of immunology (Baltimore, Md. : 1950) 158, 5841-5848.

Morotomi-Yano, K., Yano, K.-i., Saito, H., Sun, Z., Iwama, A., and Miki, Y. (2002). Human regulatory factor X 4 (RFX4) is a testis-specific dimeric DNA-binding protein that cooperates with other human RFX members. The Journal of biological chemistry $277,836-842$.

Nair, A., Frederick, T.J., and Miller, S.D. (2008). Astrocytes in multiple sclerosis: a product of their environment. Cellular and molecular life sciences : CMLS 65, 2702-2720.

Naumann, W. (1986). Immunhistochemische Untersuchungen zur Ontogenese des Subcommissuralorgans. Acta histochemica. Supplementband 33, 265-272.

Nedergaard, M., Ransom, B., and Goldman, S.A. (2003). New roles for astrocytes: redefining the functional architecture of the brain. Trends in neurosciences 26, 523-530. 
Nelander, J., Hebsgaard, J.B., and Parmar, M. (2009). Organization of the human embryonic ventral mesencephalon. Gene expression patterns : GEP 9, 555-561.

Newberne, P.M. (1962). The subcommissural organ of the vitamin B12-deficient rat. The Journal of nutrition 76, 393-413.

Nualart, F., Hein, S., Rodríguez, E.M., and Oksche, A. (1991). Identification and partial characterization of the secretory glycoproteins of the bovine subcommissural organ-Reissner's fiber complex. Evidence for the existence of two precursor forms. Molecular Brain Research 11, 227-238.

Nygårdas, P.T., Määttä, J.A., and Hinkkanen, A.E. (2000). Chemokine expression by central nervous system resident cells and infiltrating neutrophils during experimental autoimmune encephalomyelitis in the BALB/c mouse. European journal of immunology 30, 1911-1918.

Ohtoshi, A. (2008). Hydrocephalus caused by conditional ablation of the Pten or beta-catenin gene. Cerebrospinal fluid research 5, 16.

Ortloff, A.R., Vío, K., Guerra, M., Jaramillo, K., Kaehne, T., Jones, H., McAllister, J.P., and Rodríguez, E. (2013). Role of the subcommissural organ in the pathogenesis of congenital hydrocephalus in the HTx rat. Cell and tissue research 352, 707-725.

Overholser, M.D., Whitley, J.R., O'Dell, B.L., and Hogan, A.G. (1954). The ventricular system in hydrocephalic rat brains produced by a deficiency of vitamin B12 or of folic acid in the maternal diet. The Anatomical record 120, 917-933.

Pasquini, L.A., Calatayud, C.A., Bertone Uña, A L, Millet, V., Pasquini, J.M., and Soto, E.F. (2007). The neurotoxic effect of cuprizone on oligodendrocytes depends on the presence of pro-inflammatory cytokines secreted by microglia. Neurochemical research 32, 279-292.

Patel, J.R., Williams, J.L., Muccigrosso, M.M., Liu, L., Sun, T., Rubin, J.B., and Klein, R.S. (2012). Astrocyte TNFR2 is required for CXCL12-mediated regulation of oligodendrocyte progenitor proliferation and differentiation within the adult CNS. Acta neuropathologica 124, 847-860.

Pérez-Fígares, J.M., Jimenez, A.J., and Rodríguez, E.M. (2001). Subcommissural organ, cerebrospinal fluid circulation, and hydrocephalus. Microscopy research and technique 52, 591-607.

Pérez-Fígares, J.M., Jiménez, A.J., Pérez-Martín, M., Fernández-Llebrez, P., Cifuentes, M., Riera, P., Rodríguez, S., and Rodríguez, E.M. (1998). Spontaneous congenital hydrocephalus in the mutant 
mouse hyh. Changes in the ventricular system and the subcommissural organ. Journal of neuropathology and experimental neurology 57, 188-202.

Pittock, S.J., and Lucchinetti, C.F. (2007). The pathology of MS: new insights and potential clinical applications. The neurologist 13, 45-56.

Polman, C.H., Reingold, S.C., Banwell, B., Clanet, M., Cohen, J.A., Filippi, M., Fujihara, K., Havrdova, E., Hutchinson, M., and Kappos, L., et al. (2011). Diagnostic criteria for multiple sclerosis: 2010 revisions to the McDonald criteria. Annals of neurology 69, 292-302.

Qin, H., and Benveniste, E.N. (2012). ELISA methodology to quantify astrocyte production of cytokines/chemokines in vitro. Methods in molecular biology (Clifton, N.J.) 814, 235-249.

Raasch, J., Zeller, N., van Loo, G., Merkler, D., Mildner, A., Erny, D., Knobeloch, K.-P., Bethea, J.R., Waisman, A., and Knust, M., et al. (2011). IkappaB kinase 2 determines oligodendrocyte loss by noncell-autonomous activation of NF-kappaB in the central nervous system. Brain : a journal of neurology 134, 1184-1198.

Ramos, C., Fernández-Llebrez, P., Bach, A., Robert, B., and Soriano, E. (2004). Msx1 disruption leads to diencephalon defects and hydrocephalus. Developmental dynamics : an official publication of the American Association of Anatomists 230, 446-460.

Ransohoff, R.M. (2012). Animal models of multiple sclerosis: the good, the bad and the bottom line. Nature neuroscience 15, 1074-1077.

Ransohoff, R.M., Hamilton, T.A., Tani, M., Stoler, M.H., Shick, H.E., Major, J.A., Estes, M.L., Thomas, D.M., and Tuohy, V.K. (1993). Astrocyte expression of mRNA encoding cytokines IP-10 and JE/MCP-1 in experimental autoimmune encephalomyelitis. FASEB journal : official publication of the Federation of American Societies for Experimental Biology 7, 592-600.

Reith, W., and Mach, B. (2001). The bare lymphocyte syndrome and the regulation of MHC expression. Annual review of immunology 19, 331-373.

Remington, L.T., Babcock, A.A., Zehntner, S.P., and Owens, T. (2007). Microglial recruitment, activation, and proliferation in response to primary demyelination. The American journal of pathology 170, 1713-1724. 
Rodriguez, S., Rodriguez, P., Banse, C., Rodriguez, E., and Oksche, A. (1987). Reissner's fiber, massa caudalis and ampulla caudalis in the spinal cord of lamprey larvae (Geotria australis). Cell Tissue Res. 247.

Rodríguez, E.M., Oksche, A., Hein, S., and Yulis, C.R. (1992). Cell biology of the subcommissural organ. International review of cytology 135, 39-121.

Rodríguez, E.M., Rodríguez, S., and Hein, S. (1998). The subcommissural organ. Microscopy research and technique $41,98-123$.

Rolf, B., Kutsche, M., and Bartsch, U. (2001). Severe hydrocephalus in L1-deficient mice. Brain Research 891, 247-252.

Schoebitz, K., Garrido, O., Heinrichs, M., Speer, L., and Rodríguez, E.M. (1986). Ontogenetical development of the chick and duck subcommissural organ. An immunocytochemical study. Histochemistry 84, 31-40.

Schönrock, L.M., Gawlowski, G., and Brück, W. (2000). Interleukin-6 expression in human multiple sclerosis lesions. Neuroscience letters 294, 45-48.

Schwaninger, M., Sallmann, S., Petersen, N., Schneider, A., Prinz, S., Libermann, T.A., and Spranger, M. (1999). Bradykinin induces interleukin- 6 expression in astrocytes through activation of nuclear factor-kappaB. Journal of neurochemistry 73, 1461-1466.

Selmaj, K.W., and Raine, C.S. (1988). Tumor necrosis factor mediates myelin and oligodendrocyte damage in vitro. Annals of neurology 23, 339-346.

Shimizu, S., Kondo, M., Miyamoto, Y., and Hayashi, M. (2002). Foxa (HNF3) Up-regulates Vitronectin Expression during Retinoic Acid-induced Differentiation in Mouse Neuroblastoma Neuro2a Cells. Cell Structure and Function 27, 181-188.

Silvestroff, L., Bartucci, S., Soto, E., Gallo, V., Pasquini, J., and Franco, P. (2010). Cuprizone-induced demyelination in CNP::GFP transgenic mice. The Journal of comparative neurology 518, 2261-2283.

Skripuletz, T., Hackstette, D., Bauer, K., Gudi, V., Pul, R., Voss, E., Berger, K., Kipp, M., Baumgärtner, W., and Stangel, M. (2013). Astrocytes regulate myelin clearance through recruitment of microglia during cuprizone-induced demyelination. Brain : a journal of neurology 136, 147-167. 
Sobel, R.A., Mitchell, M.E., and Fondren, G. (1990). Intercellular adhesion molecule-1 (ICAM-1) in cellular immune reactions in the human central nervous system. The American journal of pathology $136,1309-1316$.

Sofroniew, M.V. (2014). Astrogliosis. Cold Spring Harbor perspectives in biology 7.

Sofroniew, M.V., and Vinters, H.V. (2010). Astrocytes: biology and pathology. Acta neuropathologica $119,7-35$.

Sousa, Vivian de Oliveira, Romão, L., Neto, V.M., and Gomes, Flávia Carvalho Alcantara (2004). Glial fibrillary acidic protein gene promoter is differently modulated by transforming growth factor-beta 1 in astrocytes from distinct brain regions. The European journal of neuroscience 19, 1721-1730.

Stadelmann, C., and Brück, W. (2008). Interplay between mechanisms of damage and repair in multiple sclerosis. Journal of neurology 255 Suppl 1, 12-18.

Stangel, M., and Hartung, H.-P. (2002). Remyelinating strategies for the treatment of multiple sclerosis. Progress in Neurobiology 68, 361-376.

Steimle, V., Durand, B., Barras, E., Zufferey, M., Hadam, M.R., Mach, B., and Reith, W. (1995). A novel DNA-binding regulatory factor is mutated in primary MHC class II deficiency (bare lymphocyte syndrome). Genes \& development 9, 1021-1032.

Sterba, G., and Wolf, G. (1969). Vorkommen und Funktion der Sialinsäure im Reissnerschen Faden. Histochemie 17, 57-63.

Sunnemark, D., Eltayeb, S., Nilsson, M., Wallström, E., Lassmann, H., Olsson, T., Berg, A.-L., and Ericsson-Dahlstrand, A. (2005). CX3CL1 (fractalkine) and CX3CR1 expression in myelin oligodendrocyte glycoprotein-induced experimental autoimmune encephalomyelitis: kinetics and cellular origin. Journal of neuroinflammation 2, 17.

Swoboda, P., Adler, H.T., and Thomas, J.H. (2000). The RFX-Type Transcription Factor DAF-19 Regulates Sensory Neuron Cilium Formation in C. elegans. Molecular Cell 5, 411-421.

Sztriha, L., Vos, Y.J., Verlind, E., Johansen, J., and Berg, B. (2002). X-linked hydrocephalus: a novel missense mutation in the L1CAM gene. Pediatric Neurology 27, 293-296. 
Takeuchi, I.K., Kimura, R., Matsuda, M., and Shoji, R. (1987). Absence of subcommissural organ in the cerebral aqueduct of congenital hydrocephalus spontaneously occurring in MT/Hokldr mice. Acta neuropathologica $73,320-322$.

Takeuchi, I.K., and Takeuchi, Y.K. (1986). Congenital hydrocephalus following X-irradiation of pregnant rats on an early gestational day. Neurobehavioral toxicology and teratology 8, 143-150.

Tanuma, N., Sakuma, H., Sasaki, A., and Matsumoto, Y. (2006). Chemokine expression by astrocytes plays a role in microglia/macrophage activation and subsequent neurodegeneration in secondary progressive multiple sclerosis. Acta neuropathologica 112, 195-204.

Tarozzo, G., Bortolazzi, S., Crochemore, C., Chen, S.-C., Lira, A.S., Abrams, J.S., and Beltramo, M. (2003). Fractalkine protein localization and gene expression in mouse brain. Journal of neuroscience research $73,81-88$.

Tchélingérian, J.-L., Monge, M., Le Saux, F., Zalc, B., and Jacque, C. (1995). Differential Oligodendroglial Expression of the Tumor Necrosis Factor Receptors In Vivo and In Vitro. Journal of neurochemistry $65,2377-2380$.

Thomas, J., Morlé, L., Soulavie, F., Laurençon, A., Sagnol, S., and Durand, B. (2010). Transcriptional control of genes involved in ciliogenesis: a first step in making cilia. Biology of the cell / under the auspices of the European Cell Biology Organization 102, 499-513.

Thompson, A. (2004). Overview of primary progressive multiple sclerosis (PPMS): similarities and differences from other forms of MS, diagnostic criteria, pros and cons of progressive diagnosis. Multiple sclerosis (Houndmills, Basingstoke, England) 10 Suppl 1, S2-7.

Toft-Hansen, H., Füchtbauer, L., and Owens, T. (2011). Inhibition of reactive astrocytosis in established experimental autoimmune encephalomyelitis favors infiltration by myeloid cells over $\mathrm{T}$ cells and enhances severity of disease. Glia 59, 166-176.

Torkildsen, O., Brunborg, L.A., Myhr, K.-M., and Bø, L. (2008). The cuprizone model for demyelination. Acta neurologica Scandinavica. Supplementum 188, 72-76.

Valerio, A. (2002). Soluble Interleukin-6 (IL-6) Receptor/IL-6 Fusion Protein Enhances in Vitro Differentiation of Purified Rat Oligodendroglial Lineage Cells. Molecular and Cellular Neuroscience 21, 602-615. 
Veto, S., Acs, P., Bauer, J., Lassmann, H., Berente, Z., Setalo, G., Borgulya, G., Sumegi, B., Komoly, S., and Gallyas, F., et al. (2010). Inhibiting poly(ADP-ribose) polymerase: a potential therapy against oligodendrocyte death. Brain : a journal of neurology 133, 822-834.

Vio, K., Rodríguez, S., Navarrete, E.H., Pérez-Fígares, J.M., Jiménez, A.J., and Rodríguez, E.M. (2000). Hydrocephalus induced by immunological blockage of the subcommissural organ-Reissner's fiber (RF) complex by maternal transfer of anti-RF antibodies. Experimental Brain Research 135, 41-52.

Voskuhl, R.R., Peterson, R.S., Song, B., Ao, Y., Morales, Laurie Beth J, Tiwari-Woodruff, S., and Sofroniew, M.V. (2009). Reactive astrocytes form scar-like perivascular barriers to leukocytes during adaptive immune inflammation of the CNS. The Journal of neuroscience : the official journal of the Society for Neuroscience 29, 11511-11522.

Voss, E.V., Škuljec, J., Gudi, V., Skripuletz, T., Pul, R., Trebst, C., and Stangel, M. (2012). Characterisation of microglia during de- and remyelination: can they create a repair promoting environment? Neurobiology of disease 45, 519-528.

Webster, H. d. F (1997). Growth factors and myelin regeneration in multiple sclerosis. Multiple Sclerosis 3, 113-120.

Williams, A., Piaton, G., and Lubetzki, C. (2007). Astrocytes--friends or foes in multiple sclerosis? Glia $55,1300-1312$.

Zeinstra, E., Wilczak, N., and Keyser, J. de (2003). Reactive astrocytes in chronic active lesions of multiple sclerosis express co-stimulatory molecules B7-1 and B7-2. Journal of Neuroimmunology 135, 166-171.

Zeinstra, E., Wilczak, N., Streefland, C., and Keyser, J. de (2000). Astrocytes in chronic active multiple sclerosis plaques express MHC class II molecules. Neuroreport 11, 89-91.

Zhang, D., Harry, G.J., Blackshear, P.J., and Zeldin, D.C. (2008). G-protein pathway suppressor 2 (GPS2) interacts with the regulatory factor X4 variant 3 (RFX4_V3) and functions as a transcriptional co-activator. The Journal of biological chemistry $283,8580-8590$.

Zhang, D., Stumpo, D.J., Graves, J.P., DeGraff, L.M., Grissom, S.F., Collins, J.B., Li, L., Zeldin, D.C., and Blackshear, P.J. (2006a). Identification of potential target genes for RFX4_V3, a transcription factor critical for brain development. Journal of neurochemistry $98,860-875$. 
Zhang, D., Zeldin, D.C., and Blackshear, P.J. (2007). Regulatory factor X4 variant 3: a transcription factor involved in brain development and disease. Journal of neuroscience research 85, 3515-3522.

Zhang, J., Williams, M.A., and Rigamonti, D. (2006b). Genetics of human hydrocephalus. Journal of neurology 253, 1255-1266. 
